University of Louisville

ThinkIR: The University of Louisville's Institutional Repository

Electronic Theses and Dissertations

$5-2014$

\title{
Three-dimensional modeling of the human jaw/teeth using optics and statistics.
}

Aly Saber Abdelrahim

University of Louisville

Follow this and additional works at: https://ir.library.louisville.edu/etd

Part of the Electrical and Computer Engineering Commons

\section{Recommended Citation}

Abdelrahim, Aly Saber, "Three-dimensional modeling of the human jaw/teeth using optics and statistics." (2014). Electronic Theses and Dissertations. Paper 3.

https://doi.org/10.18297/etd/3

This Doctoral Dissertation is brought to you for free and open access by ThinkIR: The University of Louisville's Institutional Repository. It has been accepted for inclusion in Electronic Theses and Dissertations by an authorized administrator of ThinkIR: The University of Louisville's Institutional Repository. This title appears here courtesy of the author, who has retained all other copyrights. For more information, please contact thinkir@louisville.edu. 


\title{
THREE-DIMENSIONAL MODELING OF THE HUMAN JAW/TEETH USING OPTICS AND STATISTICS
}

\author{
By \\ Aly Saber Abdelrahim \\ M.Sc., EE, Assiut University, Egypt, 2007
}

\begin{abstract}
A Dissertation
Submitted to the Faculty of the

J. B. Speed School of the University of Louisville in Partial Fulfillment of the Requirements

for the Degree of
\end{abstract}

Doctor of Philosophy

Department of Electrical and Computer Engineering

University of Louisville

Louisville, Kentucky

May, 2014 

THREE-DIMENSIONAL MODELING OF THE HUMAN JAW/TEETH USING OPTICS AND STATISTICS

$$
\text { By }
$$

Aly Saber Abdelrahim

M.Sc., EE, Assiut University, Egypt, 2007

A Dissertation Approved on

April 16, 2014

by the Following Reading and Examination Committee:

\author{
Aly A. Farag, Ph.D., Dissertation Director \\ James H. Graham, Ph.D. \\ Prasanna K. Sahoo, Ph.D.
}

Tamer Inanc, Ph.D.

Amirali Zandinejad, DDS;M.Sc. 


\section{DEDICATION}

I would like to dedicate this dissertation to my beloved wife Sally for her love and patience. 


\section{ACKNOWLEDGMENTS}

I would like to express my deepest gratitude to my advisor Dr. Aly A. Farag for his guidance, support, and encouragement to pursue a Ph.D. degree in the computer vision and medical imaging field.

I would like to acknowledge the other members of my Ph.D. committee for spending time and effort in reading and reviewing my work Dr. James H. Graham, Dr. Prasanna K. Sahoo, Dr. Tamer Inanc, and Dr. Amirali Zandinejad.

I would also like to thank Dr. Hossam Abdelmunim and Dr. Moumen Elmelegy with whom I enjoyed very fruitful and stimulating research collaborations. Our discussions were always a great pleasure, and led to significant improvements in the content and exposition of this work. They gave me important and fruitful guidance during my first steps into $3 \mathrm{D}$ reconstruction.

Also, I would like to thank to Mr. Mike Miller, and all CVIP Lab members for their friendship and assistance. I am especially grateful to Shireen Elhabian, Marwa Ismail, Ahmed Shalaby, Ahmed Elbarkouky, Mostafa Abdelrahman, Eslam Mostafa and Ali Helmi.

Finally, words cannot describe how I am indebted to my wife Sally, my sons Yousef and Moaaz and my family for their pain, suffering and sacrifices made during the journey of this study. 


\begin{abstract}
THREE-DIMENSIONAL MODELING OF THE HUMAN JAW/TEETH USING OPTICS AND STATISTICS
\end{abstract}

Aly Saber Abdelrahim

April 16, 2014

Object modeling is a fundamental problem in engineering, involving talents from computeraided design, computational geometry, computer vision and advanced manufacturing. The process of object modeling takes three stages: sensing, representation, and analysis. Various sensors may be used to capture information about objects; optical cameras and laser scanners are common with rigid objects, while X-ray, CT and MRI are common with biological organs. These sensors may provide a direct or an indirect inference about the object, requiring a geometric representation in the computer that is suitable for subsequent usage. Geometric representations that are compact, i.e., capture the main features of the objects with a minimal number of data points or vertices, fall into the domain of computational geometry. Once a compact object representation is in the computer, various analysis steps can be conducted, including recognition, coding, transmission, etc.

The subject matter of this dissertation is object reconstruction from a sequence of optical images using shape from shading (SFS) and SFS with shape priors. The application domain is dentistry. Most of the SFS approaches focus on the computational part of the SFS problem, i.e. the numerical solution. As a result, the imaging model in most conventional SFS algorithms has been simplified under three simple, but restrictive assumptions: (1) the 
camera performs an orthographic projection of the scene, (2) the surface has a Lambertian reflectance and (3) the light source is a single point source at infinity. Unfortunately, such assumptions are no longer held in the case of reconstruction of real objects as intra-oral imaging environment for human teeth. In this work, we introduce a more realistic formulation of the SFS problem by considering the image formation components: the camera, the light source, and the surface reflectance.

This dissertation proposes a non-Lambertian SFS algorithm under perspective projection which benefits from camera calibration parameters. The attenuation of illumination is taken account due to near-field imaging. The surface reflectance is modeled using the Oren-Nayar-Wolff model which accounts for the retro-reflection case. In this context, a new variational formulation is proposed that relates an evolving surface model with image information, taking into consideration that the image is taken by a perspective camera with known parameters. A new energy functional is formulated to incorporate brightness, smoothness and integrability constraints. In addition, to further improve the accuracy and practicality of the results, 3D shape priors are incorporated in the proposed SFS formulation. This strategy is motivated by the fact that humans rely on strong prior information about the 3D world around us in order to perceive 3D shape information. Such information is statistically extracted from training 3D models of the human teeth.

The proposed SFS algorithms have been used in two different frameworks in this dissertation: a) holistic, which stitches a sequence of images in order to cover the entire jaw, and then apply the SFS, and b) piece-wise, which focuses on a specific tooth or a segment of the human jaw, and applies SFS using physical teeth illumination characteristics. To augment the visible portion, and in order to have the entire jaw reconstructed without the use of CT or MRI or even X-rays, prior information were added which gathered from a database of human jaws. This database has been constructed from an adult population with variations in teeth size, degradation and alignments. The database contains both shape and albedo information for the population. Using this database, a novel statistical shape from 
shading (SSFS) approach has been created.

Extending the work on human teeth analysis, Finite Element Analysis (FEA) is adapted for analyzing and calculating stresses and strains of dental structures. Previous Finite Element (FE) studies used approximate 2D models. In this dissertation, an accurate three-dimensional CAD model is proposed. 3D stress and displacements of different teeth type are successfully carried out. A newly developed open-source finite element solver, Finite Elements for Biomechanics (FEBio), has been used. The limitations of the experimental and analytical approaches used for stress and displacement analysis are overcome by using FEA tool benefits such as dealing with complex geometry and complex loading conditions. 


\section{TABLE OF CONTENTS}

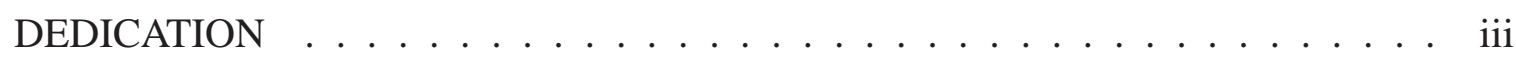

ACKNOWLEDGMENTS . . . . . . . . . . . . . . . . . iv

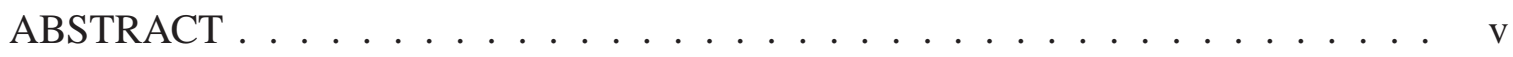

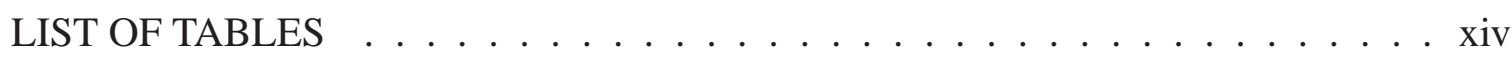

LIST OF FIGURES . . . . . . . . . . . . . . . . . . . . Xv

LIST OF ALGORITHMS . . . . . . . . . . . . . . . . . . . xxi

CHAPTER

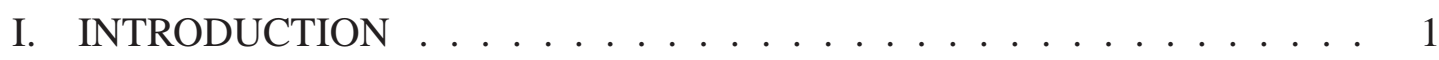

A. Reconstruction from Sequence of Images . . . . . . . . . . . . 3

1. $3 \mathrm{D}$ from Images $\ldots \ldots \ldots$

2. Camera Field of View .............. . . . 8

a. Orthogonal Projection: . . . . . . . . . . . . 8

b. Perspective Projection: . . . . . . . . . . . . . 9

3. Camera-Externally and Internally . . . . . . . . . . . 9

B. The General Form of Perspective Projection Matrix . . . . . . . . . . . 10

1. Extrinsic Parameters . . . . . . . . . . . . . . . . 11

2. Intrinsic Parameters . . . . . . . . . . . . 11

C. Camera Calibration ....................... 12

1. Why is camera calibration necessary? . . . . . . . . . 12 
2. Calibration Schema . . . . . . . . . . . 12

3. Linear Approach to Camera Calibration . . . . . . . . . . 13

a. Projection Matrix Estimation: . . . . . . . . . . . . 14

D. Teeth Anatomy . . . . . . . . . . . . . . . . . . 15

1. SURFACES AND RIDGES . . . . . . . . . . . . . . . . 18

2. The periodontal ligament . . . . . . . . . . . . 18

E. Reconstruction of the Human Jaw . . . . . . . . . . . . . . . 18

F. Contributions of this Dissertation . . . . . . . . . . . 20

G. Organization of the Dissertation ............. 23

1. Chapter I . . . . . . . . . . . . . . . . 23

2. Chapter II . . . . . . . . . . . . . . . . . . 23

3. Chapter III . . . . . . . . . . . . . . . . 23

4. Chapter IV . . . . . . . . . . . . . . . . . . . . . . 24

5. Chapter V ............................ 24

6. Chapter VI ......................... 24

7. Chapter VII . . . . . . . . . . . . . . . . . . . 25

8. Chapter VIII . . . . . . . . . . . . . . . 25

9. Chapter IX . . . . . . . . . . . . . . . . . . . 25

II. SURFACE RECONSTRUCTION BY GEOMETRIC COMPUTER VISION 26

A. Shape from Shading $(\mathrm{SFS}) \ldots \ldots . \ldots . . \ldots 27$

B. Geometric Stereo . . . . . . . . . . . . . . . . 30

C. Space Carving ....................... 31

D. Integration of Reconstruction Methods . . . . . . . . . . . . 32

E. Image Formation Model . . . . . . . . . . . . . . . 33 
F. Image Irradiance . . . . . . . . . . . . . . . . 33

1. Lambertian Reflection Model . . . . . . . . . . . . . . 34

2. Oren-Nayar Diffuse Reflection Model . . . . . . . . . . . . 35

III. DENTAL DATABASE CONSTRUCTION . . . . . . . . . . . . . . 37

A. Introduction . . . . . . . . . . . . . 37

B. Tooth Database Construction . . . . . . . . . . . . . . . 37

C. Iterative Closest Point (ICP) . . . . . . . . . . . . . . 38

D. Jaw Database Construction . . . . . . . . . . . . . . . . . . 40

E. Summary ........................... 44

IV. OCCLUSAL SURFACE RECONSTRUCTION OF HUMAN TEETH FROM

A SINGLE IMAGE . . . . . . . . . . . . . . . . . . . . . 4 45

A. Introduction . . . . . . . . . . . . . . 45

B. Image Irradiance Equation $\ldots \ldots \ldots$. . . . . . . . . . 51

C. Parametric SFS for Non-Lambertian Surfaces . . . . . . . . . . . . . 52

D. SFS Minimization Functional . . . . . . . . . . . 53

E. Experimental Results . . . . . . . . . . . . . . . . . . . 54 54

1. Testing Images Panel . . . . . . . . . . . . . . . . . . 54

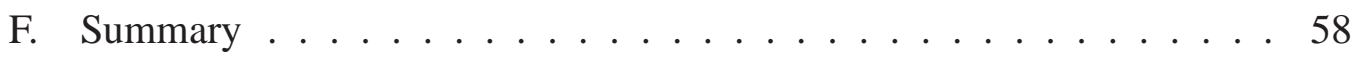

V. 3D RECONSTRUCTION OF THE HUMAN JAW USING VARIATIONAL SFS AND FEATURE DESCRIPTORS . . . . . . . . . . . . . . . 62

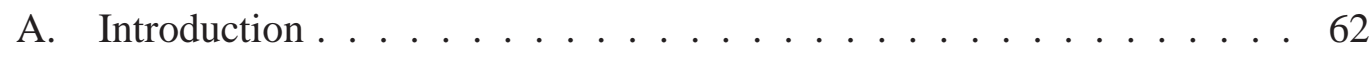

B. Data Acquisition . . . . . . . . . . . . . . 63

C. Affine Scale-Invariant Feature Transform $(A S I F T) \ldots . . . . . . .65$

D. EulerLagrange Equation .................. 66

E. Shape from shading using calibrated images . . . . . . . . . . 68 
F. The functional to be minimized $\ldots \ldots \ldots 70$

1. The Brightness constraint . . . . . . . . . . . . 70

2. The Smoothness constraint . . . . . . . . . . . . . 71

3. The Integrability constraint $\ldots \ldots \ldots . \ldots 71$

4. Variational approach . . . . . . . . . . . . 71

G. Experimental Results and Validation . . . . . . . . . . . . . 72

H. Summary ............................ 80

VI. REALISTIC 3D RECONSTRUCTION OF THE HUMAN TEETH USING

SFS WITH SHAPE PRIORS . . . . . . . . . . . . . . . . . . 8 81

A. Introduction . . . . . . . . . . . . . . 81

B. Shape Priors ... . . . . . . . . . . . . . . . . 84

C. Shape from Shading with Shape Priors . . . . . . . . . . . . 85

1. Simple Lambertian Case . . . . . . . . . . . . . . . . . . 86

2. Realistic Oren-Nayar case . . . . . . . . . . . . . 88

D. Experimental Results . . . . . . . . . . . . . . . . . . . . 89

E. Summary ............................ 91

VII. 2D-PRINCIPLE COMPONENTS ANALYSIS SHAPE MODELS . . . . . . 95

A. Introduction . . . . . . . . . . . . . . 95

B. Image Irradiance Equation . . . . . . . . . . . . . . . . . . . 98

C. Method ............................ 99

1. Data Preprocessing . . . . . . . . . . . . . . . . . 99

2. Shape Model Construction . . . . . . . . . . . . . . . . . . 99

D. Integration Tooth Shape Priors into SFS-framework . . . . . . . . . . 101

E. Experimental Results . . . . . . . . . . . . . . . . . 103 
1. Reconstruction of the Single Tooth . . . . . . . . . . . 103

2. Reconstruction of the Whole Jaw . . . . . . . . . . . 106

F. Summary . . . . . . . . . . . . . . . . . . 112

VIII. THREE-DIMENSIONAL MODELING AND STRESS ANALYSIS IN DENTAL BIOMECHANICS USING FINITE ELEMENT ANALYSIS . . . . . . 113

A. Introduction . . . . . . . . . . . . . . 113

B. Orthodontic Tooth Movement . . . . . . . . . . . . . . . 116

C. The Finite Element Methods . . . . . . . . . . . . . . . . . . . . 119

1. FEM Notation . . . . . . . . . . . . . . . . 120

2. Physical Problems, Mathematical Models and Finite Element Solution . . . . . . . . . . . . . 120

D. Equations for Three-Dimensional Solids . . . . . . . . . . . . . . . 121

1. Stress and Strain . . . . . . . . . . . . . . . 121

2. Constitutive Equation . . . . . . . . . . . . . 125

3. Dynamic Equilibrium Equation . . . . . . . . . . . . 129

4. Boundary Conditions . . . . . . . . . . . . . . . . 130

E. Materials and Methods . . . . . . . . . . . . . . . . . 131

1. Mesh generation(Pre-processing) . . . . . . . . . . . 131

2. FEA using FEBio . . . . . . . . . . . . . . 133

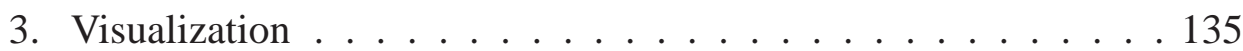

F. Experimental Results and Discussions . . . . . . . . . . . . 135

1. Horizontal Forces . . . . . . . . . . . . . . . 135

2. Vertical Forces . . . . . . . . . . . . . . . . 136

G. Summary . . . . . . . . . . . . . . . . . . 138

IX. CONCLUSIONS AND FUTURE WORK . . . . . . . . . . . . . . . 142 
A. Summary of Contributions . . . . . . . . . . . . . . . . 142

B. Limitations and Suggested Future Directions . . . . . . . . . . 143

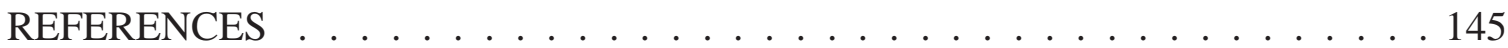

APPENDIX

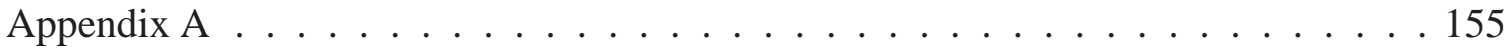

A. Derivatives of the 3 D point vector . . . . . . . . . . . 155

B. Brightness Constraint Derivatives . . . . . . . . . . . . . 157

1. Smoothness Constraint Derivatives . . . . . . . . . . . 159

C. Integrability Constraint Derivatives . . . . . . . . . . . . . 159

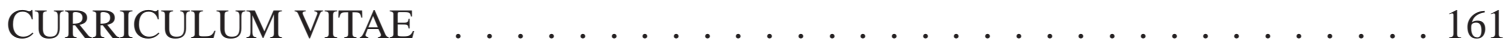




\section{LIST OF TABLES}

TABLE.

1. Database construction of the human jaw: subjects categorized with respect to gender, age and ethnicity . . . . . . . . . . . . . . 41

2. SFS Algorithm Panel . . . . . . . . . . . . . . . . . . . 56

3. Overall surface reconstruction accuracy $(R M S)$ in $m m \ldots \ldots$. . . . . . . . 58

4. Summary of approaches under experimental comparison. . . . . . . . . . . . 90

5. Summary of approaches under experimental comparison. All algorithm are a perspective camera projection and the light source at the camera optical center. . 104

6. Average tooth surface reconstruction accuracy $(R M S)$ in $m m$. . . . . . . . . 104

7. Summary of approaches under experimental comparison. . . . . . . . . . . 109

8. Average whole jaw surface reconstruction $\operatorname{accuracy}(R M S)$ in $m m . . .110$

9. Mechanical properties of human teeth (Youngs modulus and poissions ratio of the tooth) $[1-4] \ldots \ldots \ldots$. . . . . . . . . . . . . . . . . . . . . . . . . . . . . . . . 


\section{LIST OF FIGURES}

FIGURE.

1. Illustration of the data acquisition setup [5]. . . . . . . . . . . . . 3

2. Basic image formation in perspective cameras (pinhole camera). . . . . . . . . 4

3. Geometric model of a pinhole camera. . . . . . . . . . . . . . . 5

4. Camera model equations (Extrinsic $\backslash$ Intrinsic parameters). . . . . . . . . . 6

5. The three-dimensional point corresponding to a specific image point is constraint to be on the associated line of sight. . . . . . . . . . . . . 6

6. 3D point can be obtained as the intersection of two (or more) line of sights. This process is called triangulation. . . . . . . . . . . . . . 7

7. Two identical objects in different positions give the same image under orthogonal projection: object $\mathrm{O}_{2}$ is much further away from the camera, than object $O_{1}$

8. The distance effect can be felt when using perspective projection: object $\mathrm{O}_{2}$ is much further away from the camera than object $O_{1}$, and its projection is smaller than that of $O_{1} \ldots \ldots \ldots \ldots \ldots$. . . . . . . . . . . . . . 10

9. Typical calibration pattern consists of two orthogonal planes of black and white grid, to facilitate feature points extraction. The world coordinate system is attached to the calibration pattern to facilitate the measurement of 3D points. . . .

10. Anatomy of the tooth. (a) intraoral left. (b) intraoral right. (c) occlusal upper: schematic overview of the distribution of the different teeth in an adult mouth. (d) intraoral center. (e) occlusal lower.

11. Schematic overview of the anatomy of the tooth and its surrounding structure: the crown is covered with enamel, root, cusp, dentin, gum, cementum, periodontal ligament, alveolar bone and apex. . . . . . . . . . . . . . . . . . . 19 
12. Scientific prototype of the dental probe at the CVIP Lab (2000-2004). A CCD camera attached to a coordinate measuring arm captures the video images used in the jaw reconstruction. . . . . . . . . . . . . . . . . . 21

13. Definitions of reflection parameters and angles. . . . . . . . . . . . 35

14. Perspective camera model for SFS. . . . . . . . . . . . . . . 36

15. Sample individual teeth from the proposed 3D teeth library: (first row) maxillary deciduous teeth. (second row) maxillary molars. (third row) mandibular molars. (fourth row) mandibular third molars.

16. Three source models are visualized in red while the corresponding targets are shown in blue. First column show initial positions. Final registration results are demonstrated in the second column. . . . . . . . . . . . . . . . . . .

17. Sample of the human jaw (pre-repair) lower and upper jaws: first column shows the 2D images, 3D scans using cone beam CT machine shows in the second column.

18. Sample of the human jaw (post-repair ) lower and upper jaws: first column shows the 2D images, 3D scans using cone beam CT machine shows in the second column.

19. Illustration of the jaw anatomical landmarks. . . . . . . . . . . . . . . . 44

20. A calibrated wireless intraoral camera equipped by a motion tracker and Perspective projection camera model. . . . . . . . . . . . . . . . . . 48

21. Local illumination model where object is illuminated in the viewing direction, i.e. retro-reflection case.

22. Surface reflectance properties where rough surfaces tend to scatter incident light as compared to smooth surfaces. Microscopic surface height variations of a posterior occlusal surface is measured by an optical surface profiler. . . . . . . . 50

23. A stylus arm with a touching probe. . . . . . . . . . . . . . . 56

24. The 32 adult human teeth. . . . . . . . . . . . . . . . . 57 
25. First row: images for three different types of human teeth (mandibular $3^{r d}$ molar, mandibular molar and maxillary molar), captured by the intra-oral camera. Second row: groundtruth occlusal surface generated from CT scanning. Third row: surface reconstruction based on algorithm A (proposed solution). Fourth row: surface reconstruction based on algorithm B. Last row: surface reconstruction based on algorithm C. Notice algorithms B and C (PDE-based) did not capture the geometric details of the occlusal surface as compared to the minimization-based algorithm A

26. First row: images for more two different types of human teeth (maxillary deciduous and premolar), captured by the intraoral camera. Second row: groundtruth occlusal surface generated from CT scanning. Third row: surface reconstruction based on algorithm A (proposed solution). Fourth row: surface reconstruction based on algorithm B. Last row: surface reconstruction based on algorithm C. Notice algorithms B and C (PDE-based) did not capture the geometric details of the occlusal surface as compared to the minimization-based algorithm A.

27. Different overlapped sub-images, results of features matching and a jaw is imaged as 9 overlapped images: (a) A sequence of overlapped images are illustrated. (b) Point correspondences as a result of the ASIFT algorithm. (c) Point correspondences are demonstrated after removing the outliers matches using RANSAC. (d) The stitching results (panoramic image) where each sub-image is boxed by a green line to show the overlap between the views. . . . . . . . . . 74

28. Three different examples to stitch two sub-images together: Point correspondences are demonstrated in (a) using the ASIFT algorithm. Correspondences after removing the outliers using RANSAC are shown in (b). Registration of the two overlapped images is depicted in $(\mathrm{c}) \ldots \ldots \ldots \ldots . \ldots 76$

29. Two different real jaws sub-images panoramic reconstruction: A sequence of sub-images is given for each case from 1 to 8 while the resulting panoramic image is given at the last row. . . . . . . . . . . . . . . . . . 78

30. Two artificial jaws 3D surface reconstructions are demonstrated: the results of using the proposed approach are shown in the middle and the results of the technique in $[6]$ are given in the right column. . . . . . . . . . . . .

31. Aligning an input image with the reference shape model. (Left) Reference image corresponding to the constructed shape model. (Middle) Sample input test image for a tooth. (Right) The test image after being aligned with the reference image. . . . . . . . . . . . . . . . . . . . .

32. The mean shapes in the constructed database of human teeth of various types: maxillary deciduous teeth, maxillary molars, mandibular molars and mandibular third molars, respectively. . . . . . . . . . . . . . . . . . . . . 8 8 
33. Tooth reconstruction from four different algorithms. First row: image acquired by the intraoral camera. Second row: ground-truth occlusal surface generated from a CT scan of the tooth. Third row: reconstruction using Algorithm AA. Fourth row: reconstruction using Algorithm BB. Fifth row: reconstruction using the proposed method (Algorithm $\mathrm{CC}$ ). Last row: reconstruction using the proposed method (Algorithm DD). Beneath each reconstruction is the rootmean-square $(R M S)$ error when compared to the ground-truth surface from CT. . 92

34. Experiment on teeth with significant fillings. First row: input test image. Second row: reconstruction by the proposed algorithm (Algorithm DD). Third row: reconstruction by Algorithm BB. . . . . . . . . . . . . . . . . 93

35. iTero commercial dental scanning system. . . . . . . . . . . . . . . 96

36. Lava commercial dental scanning system. . . . . . . . . . . . . . . . . 97

37. Tooth reconstruction from three different algorithms. (a) 2D-image captured by the intraoral camera.(b) GT occlusal surface generated from a CT scan of the tooth. (c) 3D Reconstruction of the human teeth using the proposed method AAA. (d) Reconstruction using Algorithm DD. (e) Reconstruction using Algorithm B(well-known SFS method). Beneath each reconstruction is the rootmean-square $(R M S)$ error. . . . . . . . . . . . . . . . . . . . 105

38. Sample reconstruction result of a lower jaw (Post-repair )from three different algorithms. (a) Image acquired by the CCD camera.(b) Ground-truth generated from a CT scan of the jaw. (c) Reconstruction using the proposed Algorithm $A_{w}$ (with shape priors). (d) Reconstruction using Algorithm $B_{w}$. (e) Reconstruction using method (Algorithm $C_{w}$ ). Beneath each reconstruction is the root-meansquare (RMS) error when compared to the ground-truth surface from CT. . . . . 108

39. Experiment on teeth with significant fillings. (a) Input test image. (b) Reconstruction by the proposed algorithm $\left(A_{w}\right)$. (c) Reconstruction by algorithm $\left(C_{w}\right)$ (with no shape priors). . . . . . . . . . . . . . . . 111

40. Overall approach of the proposed method. . . . . . . . . . . . . . 114

41. Finite element geometries: First row: 1D. Second row: 2D. Third row: 2D. Fourth row: 3D. . . . . . . . . . . . . . . . . . . 121

42. The flowchart of the process of finite element analysis: (a) The physical problem. (b) Finite element solution of mathematical model. (c) postprocessing step: interpretation the results, design improvements and structural optimization. 122

43. Solid tooth subjected to forces applied within the solid (body force) and on the surface of the solid tooth $($ surface force $) \ldots \ldots \ldots . \ldots \ldots$ 
44. Six independent stress components at a point in a solid viewed on the surfaces of an infinitely small cubic block. . . . . . . . . . . . . . . . . . . . . 127

45. Stresses on an infinitely small block. Equilibrium equations are derived based on this state of stresses. . . . . . . . . . . . . . . . . . . . . . . 128

46. Ensemble of 20 models generated using the proposed method illustrated with different colors. First row: solid volumes generated from the CT scan of the anterior teeth. Second row: solid volumes generated from the CT scan of the mandibular third molars. Third row: solid volumes generated from the CT scan of the mandibular molars. Last row: solid volumes generated from the CT scan of the maxillary third molars . . . . . . . . . . . . . . . . . . . . 132

47. (a) Finite element mesh (solid mesh) with the material property and initial conditions. (b) Effective stress for the tooth due to orthodontic forces were depicted as concentrated force. (c) The correspondence total displacement for the anterior tooth . . . . . . . . . . . . . . . . . . . . . . . 137

48. The mechanism of transferring the load from one tooth to its neighborhood: (a) Finite element meshes with the material property and initial conditions. (b) The effective stress (expressed in MPa) due to the force $(3 \mathrm{~N})$ on the left tooth in the opposite y-direction and the pressure of the right tooth on the left tooth. (c) The correspondence total displacement. . . . . . . . . . . . . . . . 138

49. 3D model and stress, displacement and compression distributions in the mandibular third molar with cusp 4 points loading. (a) Finite element mesh (solid mesh) with the material property and initial conditions. (b) Effective stress for the tooth due to orthodontic $200 \mathrm{~N}$ tensile forces were depicted as four concentrated force in $-z$-direction (as a tensile force). (c) The correspondence total displacement. (d) The correspondence compression. . . . . . . . . . . . . . . . . 139

50. 3D model and stress, displacement and compression distributions in the mandibular molar (three roots) with cusp 3 points loading. (a) Finite element mesh (solid mesh) with the material property and initial conditions. (b) Effective stress for the tooth due to orthodontic $200 \mathrm{~N}$ tensile forces were depicted as four concentrated force (in $z$-direction (as a compression force). (c) The correspondence total displacement. (d) The correspondence compression.

51. 3D model and stress, displacement and compression distributions in the mandibular molar (three roots) with cusp 4 points loading. (a) Finite element mesh (solid mesh) with the material property and initial conditions. (b) Effective stress for the tooth due to orthodontic $200 \mathrm{~N}$ tensile forces were depicted as four concentrated force in $z$-direction (as a compression force). (c) The correspondence total displacement. (d) The correspondence compression. 
52. 3D model and stress, displacement and compression distributions in the maxillary 3rd molar (two roots) with cusp 3 points loading. (a) Finite element mesh (solid mesh) with the material property and initial conditions. (b) Effective stress for the tooth due to orthodontic $200 \mathrm{~N}$ tensile forces were depicted as four concentrated force in $-z$-direction (tensile force). (c) The correspondence total displacement. (d) The correspondence compression.

53. 3D model and stress, displacement and compression distributions in the maxillary 3rd molar (two roots) with cusp 4 points loading. (a) Finite element mesh (solid mesh) with the material property and initial conditions. (b) Effective stress for the tooth due to orthodontic $200 \mathrm{~N}$ tensile forces were depicted as four concentrated force in $-z$-direction. (c) The correspondence total displacement.

(d) The correspondence compression. . . . . . . . . . . . . . . . . . . . 141 


\section{LIST OF ALGORITHMS}

ALGORITHM.

PAGE

1. ICP Algorithm . . . . . . . . . . . . . . . . . 41

2. Parametric SFS under near imaging conditions . . . . . . . . . . . 55

3. New variational SFS . . . . . . . . . . . . . . . 73 


\section{CHAPTER I}

\section{INTRODUCTION}

Shape from shading (SFS) is a problem that has been studied for about four decades in the vision literature. Stated succinctly, the problem is to recover surface orientation from local variations in measured brightness. There is strong psychophysical evidence for its role in surface perception and recognition. Since the problem is an ill-posed one, a number of additional, simplifying model assumptions have been imposed in order to render it tractable.

The investigation of the SFS problem was pioneered by Horn [7]. He formulated the problem by a nonlinear first order partial differential equation (PDE) called the image irradiance equation. This equation models the relationship between the shape of an object and its image brightness under known illumination conditions. His orthographic camera model, distant single point light source, and his Lambertian surface assumption became characteristic for numerous early SFS algorithms; see e.g. [8] for a survey.

Unfortunately, those assumptions are not always valid in reality. As such, the reconstruction results of these classical SFS approaches lack accuracy. That is why there have been more recent methods trying to relax some of these assumptions. For example, Prados et al. [9], Tankus et al. [10], and Yuen et al. [11] replaced the orthographic camera model by a pinhole camera model performing a perspective projection (see Figure 2), and assumed that the light source is located at the optical centre. Moreover, a light attenuation term is considered in [9]. These ideas have been further extended by Ahmed et al. [12] and by Vogel et al. $[13,14]$. In these works, the Lambertian reflectance model is replaced by 
the more realistic model of Oren and Nayar [15], which is particularly useful for skin surfaces. The Wolff reflectance model was also investigated [16]. On the other hand, various optimization techniques have been employed to solve the SFS problem, see e.g. [17] for a recent survey.

The observation underpinning this dissertation is that although considerable effort has gone into the development of improved SFS methods, there are two areas which leave scope for further development. The first of these the incorporation of the camera calibration in the more realistic reflectance model. The second is that incorporation of $3 \mathrm{D}$ shape priors in the recovery of the unknown surface. This is quite useful specially when the target application deals with a particular category of object surfaces.

To further motivate the contribution of this work, a dental application is considered: 3D jaw/teeth reconstruction from intra-oral images. Dentistry usually requires accurate 3D representation of the teeth and jaw for diagnostic and treatment purposes. Toward this end, photogrammetry seems to offer a more convenient, cost-effective technique compared to traditional techniques while avoiding the need for castings. Computer Vision and Image Processing laboratory (CVIP lab.) research group have been interested in this SFS paradigm for jaw/teeth reconstruction from an intraoral image for several years (see for example $[6,18-21])$ due to the significant shading cue in an image of a tooth. In addition, other 3D reconstruction methods, such as Shape from Stereo, will have little success when applied on the textureless tooth surfaces [19].

In this dissertation, a novel strategies to improve the surface recovery results of SFS have been approved. 3D shape priors in the SFS formulation are incorporated. Since the target application is the human teeth reconstruction from intra-oral images, such information is statistically extracted from training 3D tooth models. This can serve in several aspects, e.g., to improve reconstruction accuracy, solve problems caused by occlusion (e.g., because of the tongue), specularity and albedo changes, and/or make up for the lack of sufficient, detailed view of a tooth. 


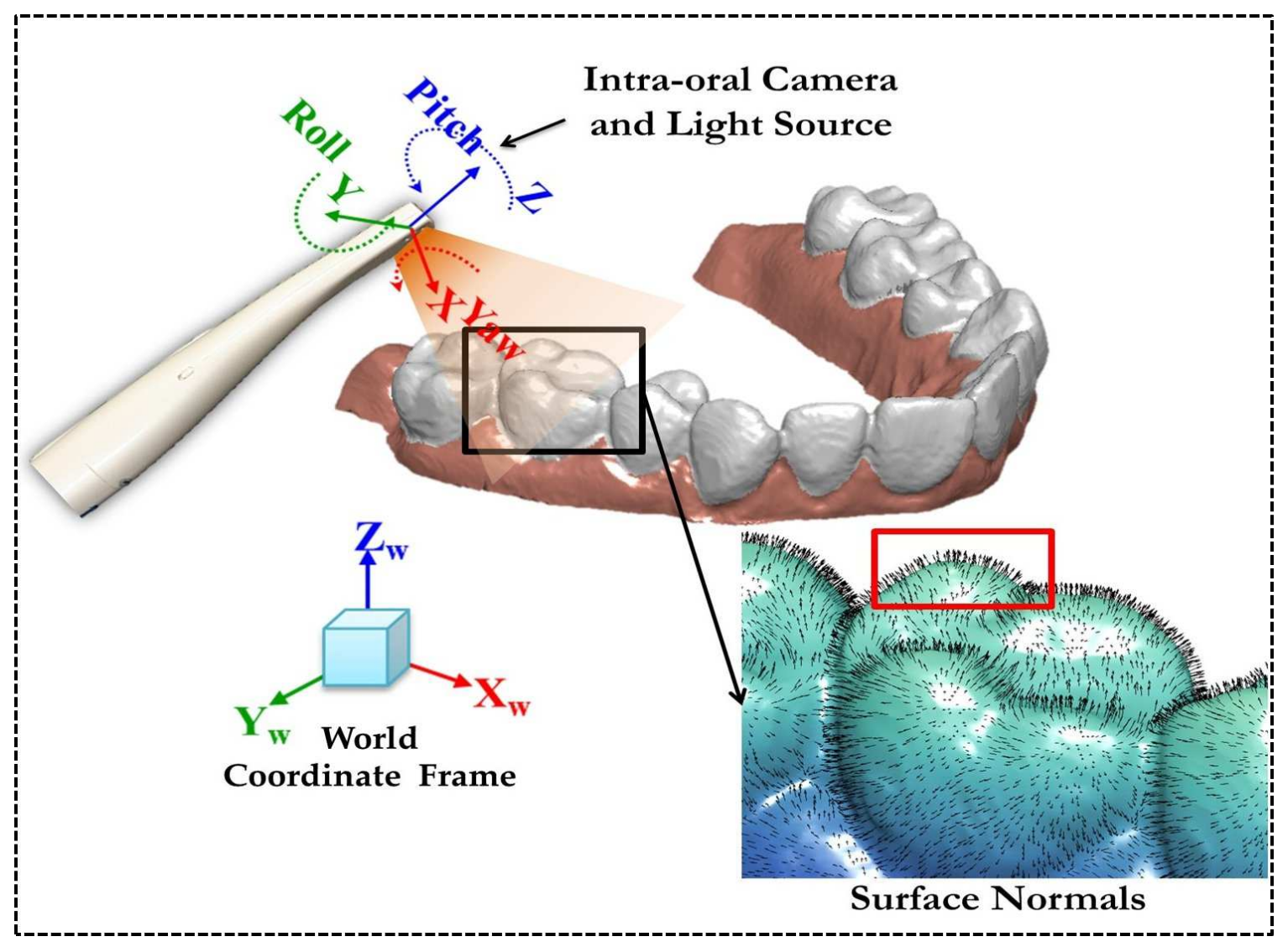

FIGURE 1: Illustration of the data acquisition setup [5].

Furthermore, we introduce a more realistic formulation of SFS that better considers all the components of the problem under concern, namely: the camera, the light source, and the surface reflectance. Since image acquisition setup consists of a small wireless intraoral camera with a built-in bright light source, the camera is modelled by perspective projection, which is more practical than the common SFS assumption of orthographic projection as the teeth are typically close to the camera (see Figure 1). The light source is assumed to be located at the optical center of the camera. Under this near-illumination imaging, we take into account the attenuation of illumination due to the distance between the light source and the surface, which helps to deal with the concave/convex ambiguity in SFS [9, 22].

\section{A. Reconstruction from Sequence of Images}




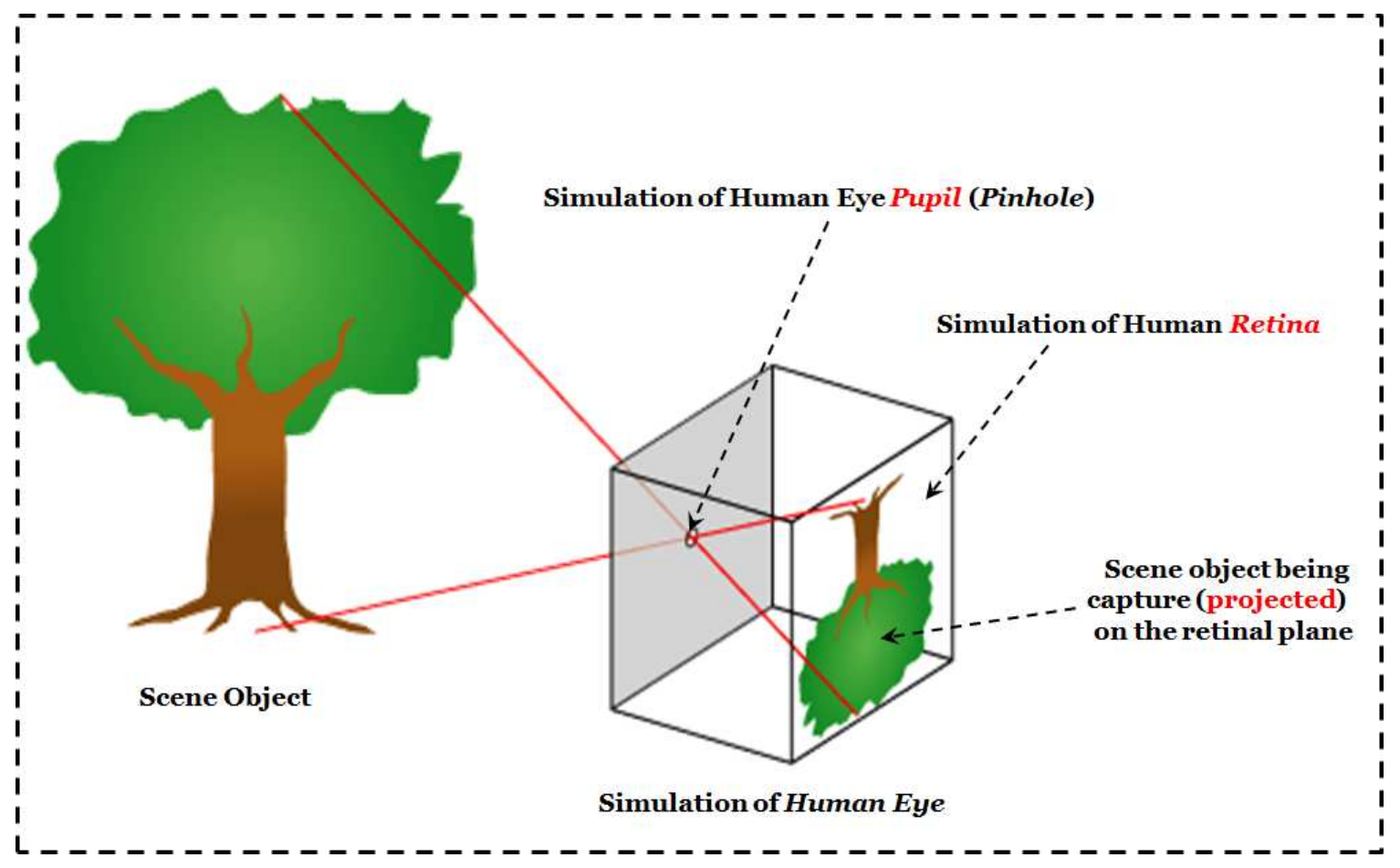

FIGURE 2: Basic image formation in perspective cameras (pinhole camera).

Optical Imaging: An optical image nowadays is formed by high-speed CCD sensors capable of capturing light reflections from an object at high spatial resolutions. Yet, at the basic level, an image may be represented as a collection of reflected light rays from an object culminated by a lens Figure 2. A pinhole camera forms inverted images of an object by rays entering a pinhole; lens at the aperture focuses the light reflected from an object such that a ray will pass unabated through the lens center, and if parallel to the lens optical axis will be deflected through the focal point. The intersection of the two rays forms an image at the image plane. Figure 3 illustrates the geometric model. The equations of the camera calibration parameters shown in Figure 4.

1. 3D from Images

An image tells a lot about the observed scene, however there is not enough information to reconstruct the whole $3 \mathrm{D}$ scene; this is due to the nature of the image formation 


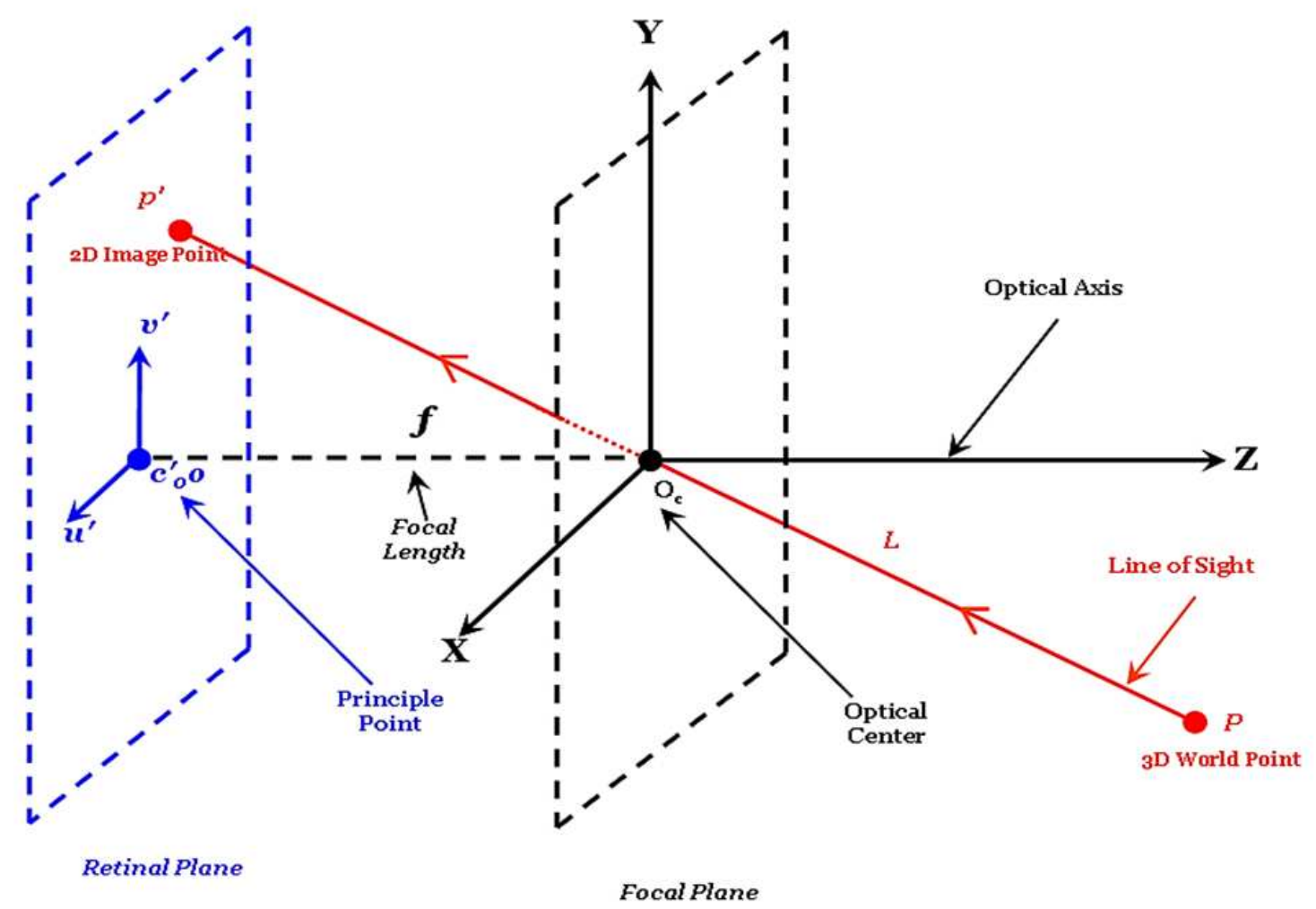

FIGURE 3: Geometric model of a pinhole camera.

process which consists of a projection from a three dimensional scene onto a two dimensional image, during this process the depth information is lost; i.e. the distance between the $3 \mathrm{D}$ object and the capturing (imaging) device. However, the three-dimensional point $P(X, Y, Z)$ corresponding to a specific image point $p(u, v)$ is constraint to be on the associated line of sight $L$, as shown in Figure 5.

Therefore, the two-dimensional point $p$ might correspond to any three-dimensional point $P$ on the line of sight $L$. Hence from a single image it is not possible to determine which point on this line corresponds to the image point. Therefore, if two or more images (views) are available for the same 3D scene (whether they are taken by different cameras or one non-stationary camera), then the $3 \mathrm{D}$ point can be obtained as the intersection of two (or more) line of sights, as illustrated in Figure 6. This process is called triangulation.

A number of things needed to accomplish such task; i.e. three-dimensional infor- 


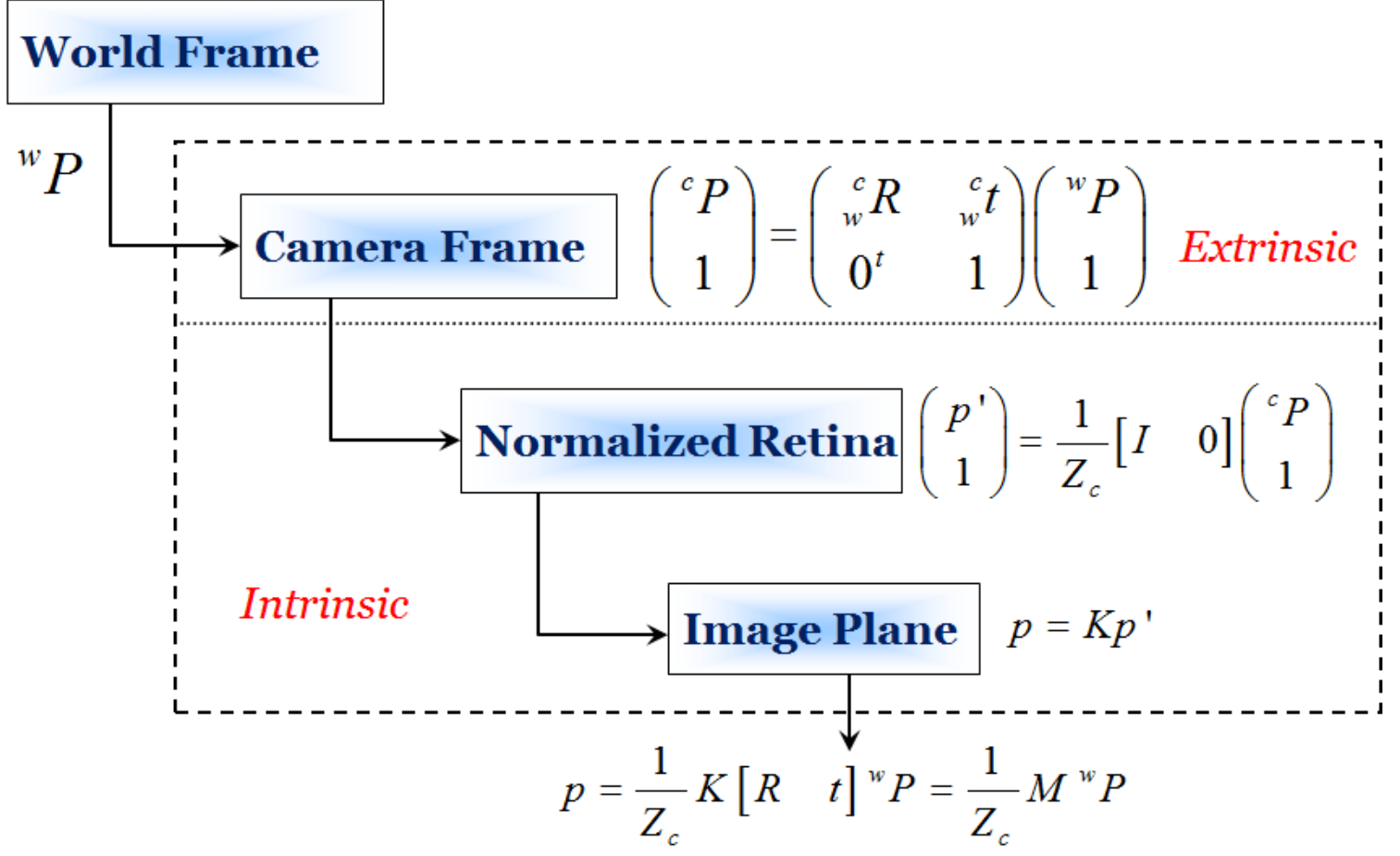

FIGURE 4: Camera model equations (Extrinsic $\backslash$ Intrinsic parameters).

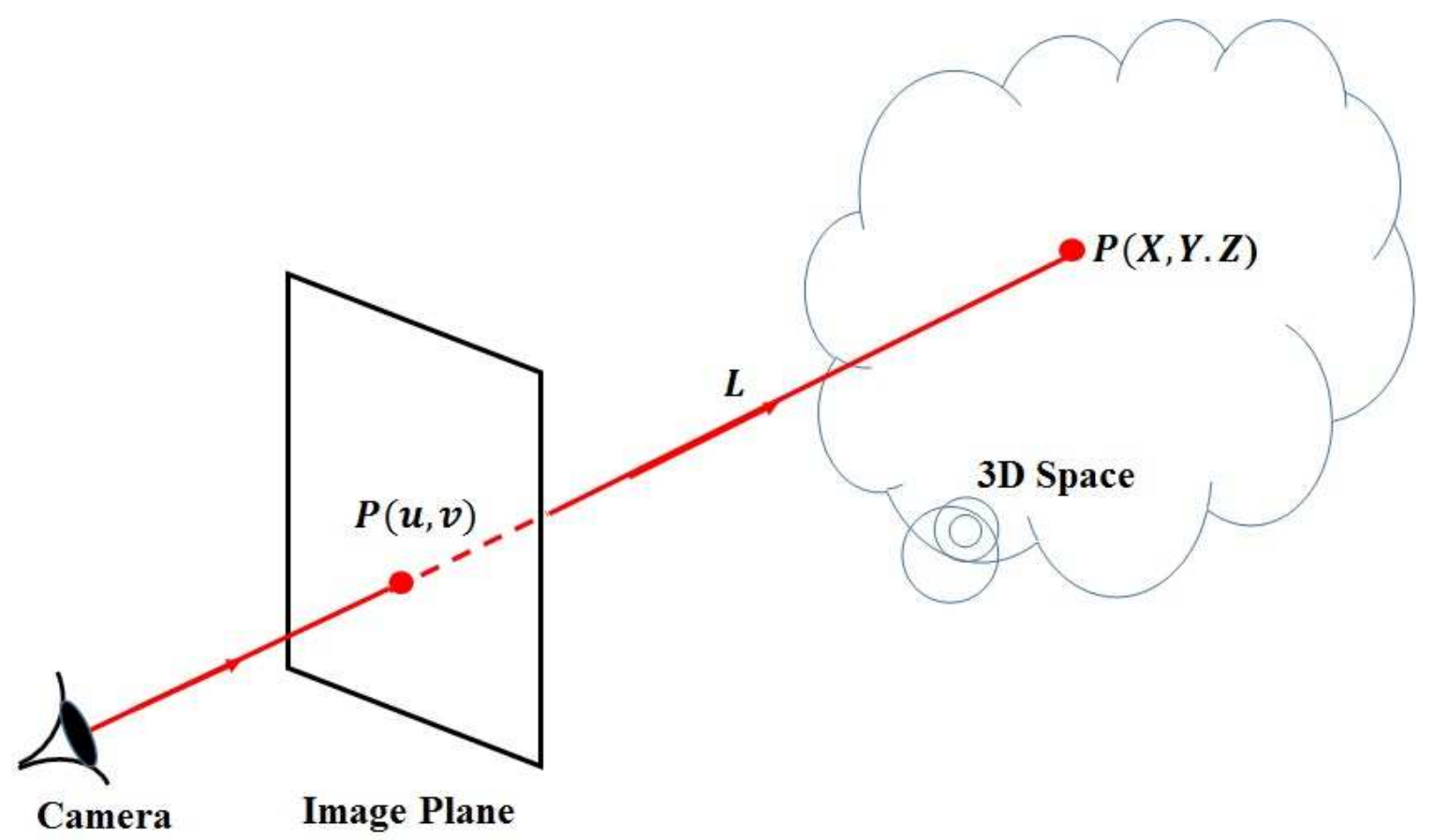

FIGURE 5: The three-dimensional point corresponding to a specific image point is constraint to be on the associated line of sight. 


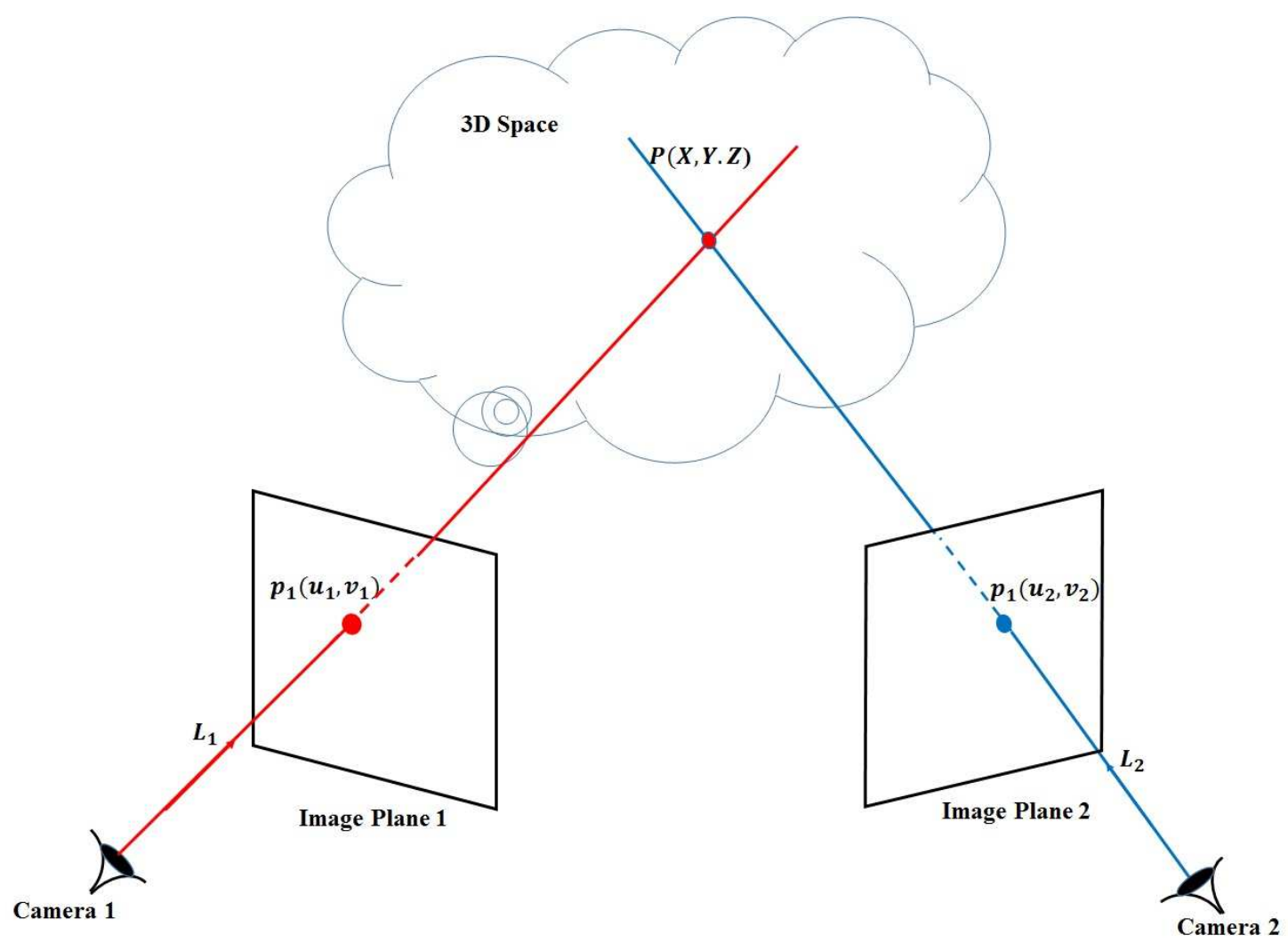

FIGURE 6: 3D point can be obtained as the intersection of two (or more) line of sights. This process is called triangulation.

mation reconstruction:

- A list of corresponding image points between different views, i.e. $p_{i}$ in view $V_{1}$ corresponds to $p_{j}$ in view $V_{2}$.

- Relative pose of the camera for the different views; i.e. the relationship between viewing cameras with respect to each other.

- The relation between the image points and the corresponding line of sights.

The relation between an image point and its line of sight is given by the so-called camera model, which answers the question of how a three-dimensional point, measured with respect to the $3 \mathrm{D}$ world coordinates, be projected on a $2 \mathrm{D}$ image plane and ends up as a 
pixel in a specific row and column, hence what is the relationship between the row/column number and the $3 \mathrm{D}$ point coordinates?

It is important here to note that using different views is not the only approach to recover or reconstruct the scene $3 \mathrm{D}$ information, shading, texture and focus cues can also be used to extract 3D information from images.

\section{Camera Field of View}

The input to the camera is a collection of 3D points which lie in the field of view of the camera. The field of view of the camera is ideally a cone, defined by its angle $£$ and its near and far plane, where the 3D points which lie between the near and far planes are the only points that can be seen by the camera. This idea was inspired by the field of view of the human eye.

Using a cone-like field of view for the camera will impose computational overhead, hence it can be approximated as a trapezoid or a cuboid (box) shape. The shape to be used depends on the type of projection considered for camera modeling. When a trapezoid is used to approximate the field of view of the camera this is called, perspective projection. While a cuboid for approximation is known as orthogonal projection.

a. Orthogonal Projection: Consider the case when using a box to approximate the camera field of view. Any 3D point that lies in the box (field of view) will be projected on the near plane (to form the 2D image) in a direction parallel to the edges of the field of view, see Figure 7 for illustration. Since the edges of the box are parallel to each other, same-size near and far object will be projected on the near and far plane having the same size. Hence methods which use orthogonal projection are only valid in a limited domain where the distance and position effects can be ignored, i.e. the objects are distant from the camera. 


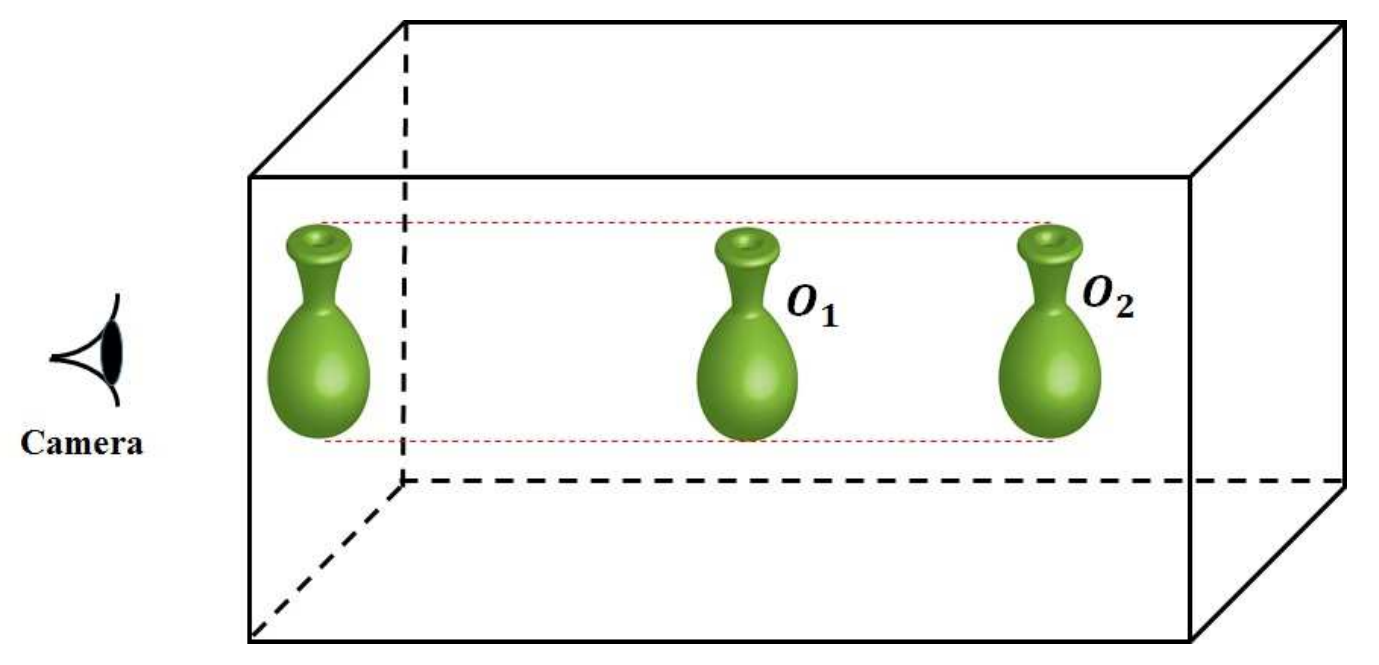

FIGURE 7: Two identical objects in different positions give the same image under orthogonal projection: object $O_{2}$ is much further away from the camera, than object $O_{1}$.

b. Perspective Projection: On the other hand, consider the case when using a trapezoid to approximate the field of view. Using a trapezoidal field of view will enable us to feel the distance effect, where far objects project smaller than near objects, keeping in mind that projection always occurs parallel to the edges of the field of view, see Figure 8 for illustration. The idea of approximating the field of view as a trapezoid is inspired by the pinhole camera model, discussed latter, which simulates the human eye.

\section{Camera-Externally and Internally}

The camera can be characterized (to the external/surrounding world) by its position, known as the optical center, its viewing direction, known as its optical axis (z-axis), its up direction (y-axis) and its right direction (x-axis). The whole idea of the camera model is inspired by the human visual system. A human being has two eyes, each characterized by there position in his/her face, i.e. the optical center, the viewing direction is defined by where the human eye is looking at, the up-direction can be viewed as the orientation of the human head with respect to the viewing direction, while the right direction maps to the humans right-hand when expanded perpendicular to the human body. 


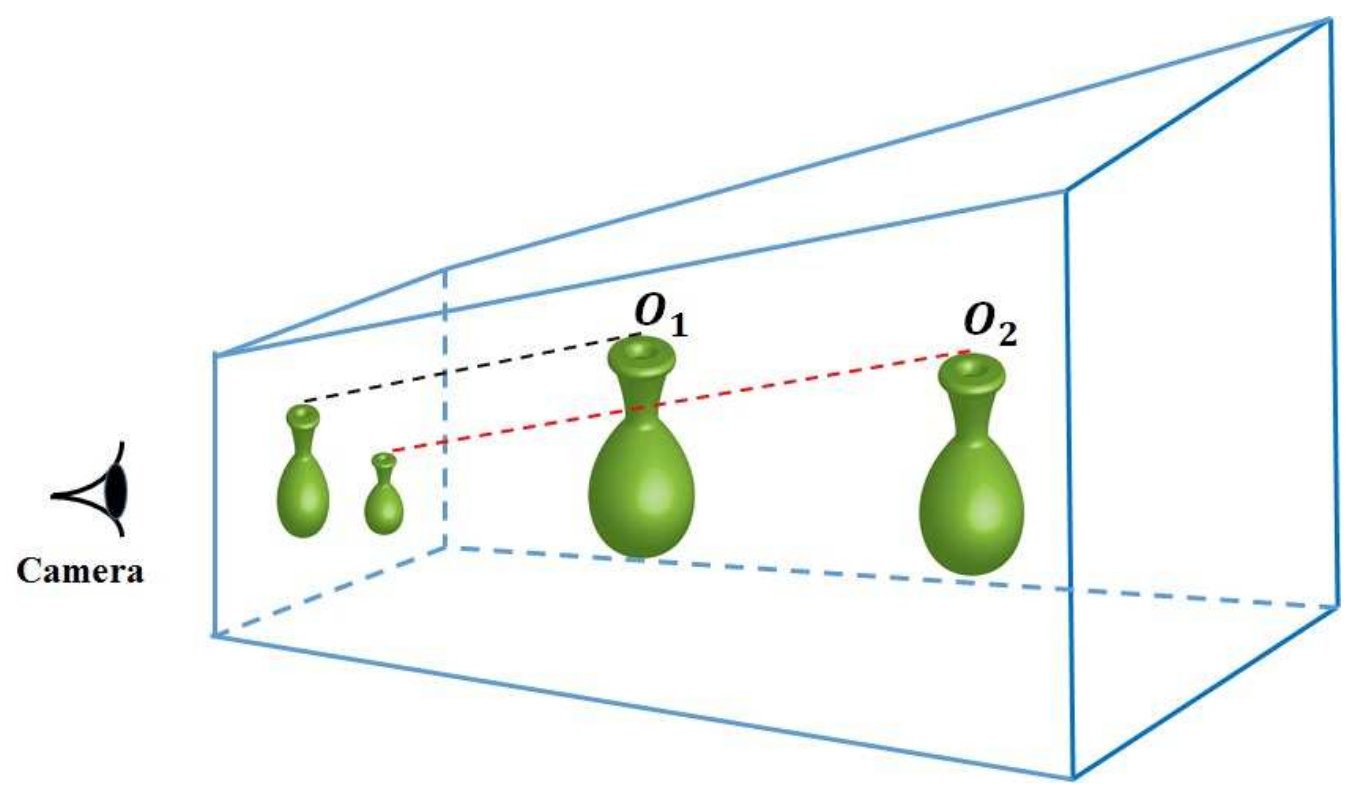

FIGURE 8: The distance effect can be felt when using perspective projection: object $O_{2}$ is much further away from the camera than object $O_{1}$, and its projection is smaller than that of $O_{1}$.

The camera can be internally characterized by its field of view, the type of projection used (i.e. the approximation of the cone-shape field of view), and the process of digitizing what is projected on the near plane to be converted to a digital image. The camera field of view is defined by the near and far plane, where the distance between the optical center and the near plane is commonly denoted as focal length.

\section{B. The General Form of Perspective Projection Matrix}

The camera can be considered as a system that depends on its parameters, which are categorized into two classes, extrinsic and intrinsic parameters. 


\section{Extrinsic Parameters}

They are the parameters related to the transformation of the world coordinate system to coincide on the camera coordinate system. There are six extrinsic parameters, the Euler angles; yaw $\alpha$, pitch $\beta$, and tilt $\gamma$ for rotation, the three components of the translation vector $t=\left(t_{x}, t_{y}, t_{z}\right)$.

The extrinsic parameters matrix, $D$, can be expressed in terms of $t$ and $R$ as:

$$
D=\left[\begin{array}{cc}
R_{3 \times 3} & t \\
0_{3}^{T} & 1
\end{array}\right]
$$

where $D$ is $4 \times 4$ matrix and $0_{3}=\left[\begin{array}{lll}0 & 0 & 0\end{array}\right]^{T}$.

\section{Intrinsic Parameters}

Those parameters are related to converting the $3 \mathrm{D}$ point coordinate measured in camera coordinate system to the computer image coordinate. There are five intrinsic parameters:

- The focal length $\alpha_{x}$ in $x$ direction and focal length $\alpha_{y}$ in $y$ direction.

- The coordinates $u_{o}, v_{o}$ of the principle point in the image plane.

- The skewing factor $s$.

$$
K=\left[\begin{array}{cccc}
\alpha_{x} & s & x_{o} & 0 \\
0 & \alpha_{y} & y_{o} & 0 \\
0 & 0 & 1 & 0
\end{array}\right]
$$


The Overall Projection Matrix (P) :

$$
P=K R\left[I_{3} \mid-\widetilde{C}\right]
$$

where $P$ is $3 \times 4$ matrix and it has 11 degree of freedom ( 5 from $K, 3$ from $R$ and 3 from $\widetilde{C}$ ).

\section{Camera Calibration}

Camera calibration is the process of determining the internal camera geometric and optical characteristics (intrinsic parameters) and/or the 3D position and orientation of the camera frame relative to a certain world coordinate system (extrinsic parameters).

1. Why is camera calibration necessary?

Camera calibration provides a way of inferring 3D information from computer image coordinates as well as inferring 2D computer image coordinate from 3D information. 3D information from computer image coordinates involves the location of the object (target) and the position and orientation of a moving camera

\section{Calibration Schema}

The key idea behind camera calibration is to write the projection equations linking the known coordinates of a set of 3D world points and their projections (image pixels coordinates) and solve for the camera parameters.

In order to get to know the coordinates of some 3D points, camera calibration methods rely on one or more images of a calibration pattern, that is a $3 \mathrm{D}$ object of known ge- 


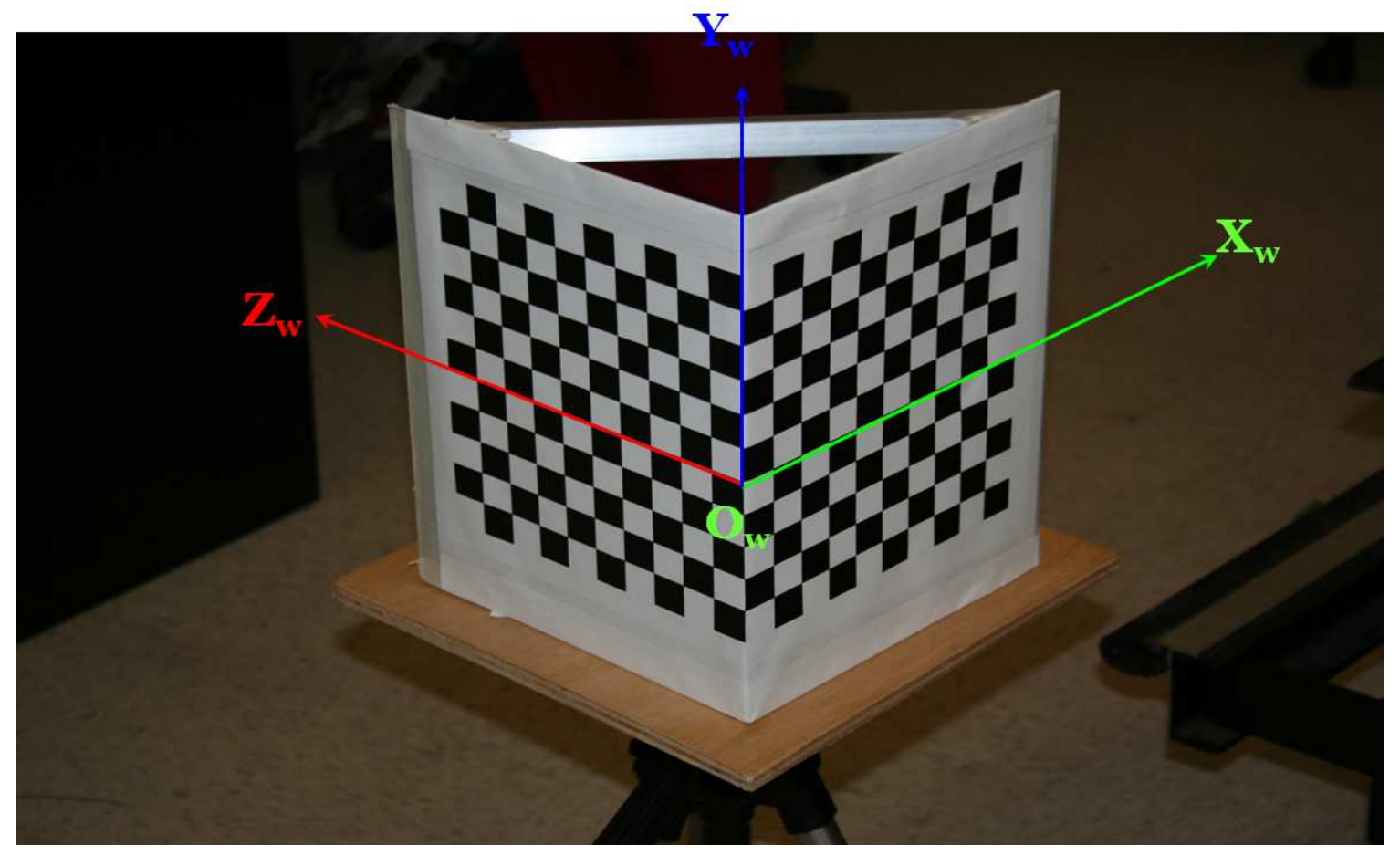

FIGURE 9: Typical calibration pattern consists of two orthogonal planes of black and white grid, to facilitate feature points extraction. The world coordinate system is attached to the calibration pattern to facilitate the measurement of 3D points.

ometry, possibly located in a known position in space and generating image features which can be located accurately. Usually a typical calibration pattern consists of two planar grids of black squares on a white background. The world coordinate system is usually attached to the calibration pattern, this will facilitate the measurement of the 3D world points, while the corresponding image points can be located using corner detection techniques, i.e. intersection of image lines, thanks to the high contrast and simple geometry of the calibration pattern, see Figure 9.

3. Linear Approach to Camera Calibration

The calibration process can be decomposed into two stages; first the computation of the perspective projection matrix, $M$, associated with the camera in this coordinate system, 
then the estimation of the intrinsic and extrinsic parameters of the camera from this matrix.

a. Projection Matrix Estimation: Assume that the projection matrix $M$, defined up to an arbitrary scale factor, is:

$$
M=\left[\begin{array}{cccc}
m_{11} & m_{12} & m_{13} & m_{14} \\
m_{21} & m_{22} & m_{23} & m_{24} \\
m_{31} & m_{32} & m_{33} & m_{34}
\end{array}\right]
$$

and given $N 3 D$ points and their corresponding $2 D$ points. Since the relation between a $3 D$ point and its corresponding $2 D$ point is:

$$
\left[\begin{array}{l}
u \\
v \\
1
\end{array}\right]=\left[\begin{array}{llll}
m_{11} & m_{12} & m_{13} & m_{14} \\
m_{21} & m_{22} & m_{23} & m_{24} \\
m_{31} & m_{32} & m_{33} & m_{34}
\end{array}\right]\left[\begin{array}{c}
x \\
y \\
z \\
1
\end{array}\right]
$$

Equation 5 indicates:

$$
\begin{gathered}
u_{i}=\frac{m_{11} x_{i}+m_{12} y_{i}+m_{13} z_{i}+m_{14}}{m_{31} x_{i}+m_{32} y_{i}+m_{33} z_{i}+m_{34}} \\
v_{i}=\frac{m_{21} x_{i}+m_{22} y_{i}+m_{23} z_{i}+m_{24}}{m_{31} x_{i}+m_{32} y_{i}+m_{33} z_{i}+m_{34}}
\end{gathered}
$$

which can be arranged in $2 N$ linear equations in $m^{\prime} s$ in the form:

$$
P m=0 \text {; }
$$

where, 


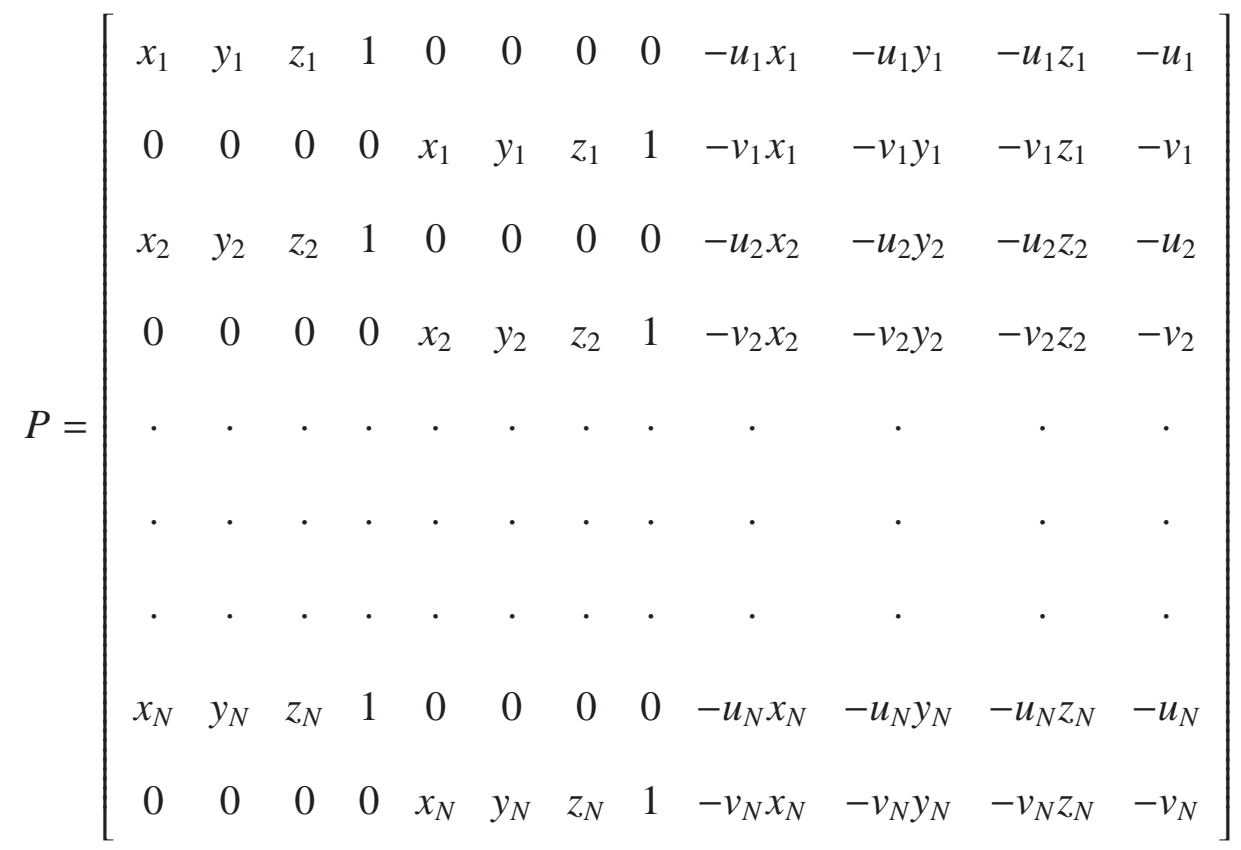

And

$$
m=\left[m_{11}, m_{12}, m_{13}, \cdots m_{33}, m_{34}\right]^{T} .
$$

then the unknown $m s$ can be recovered by the decomposition of $P$ using singular value decomposition as:

The solution is the eignvector $V$ corresponds to the smallest singular value (related to the smallest eignvalue) in the main diagonal of $D$.

$$
P=U D V^{T}
$$

\section{Teeth Anatomy}

Humans have two types of teeth in their lifetime. The first type of teeth that appears in the mouth are the primary or deciduous dentition, which begin to form prenatally at about 14 weeks in utero and are completed postnatally at the age of 3 . When there are no signs of congenital disorders, dental disease, or trauma, the first teeth begin to appear in the 
oral cavity at the age of 6 , and the last emerge at the age of $28 \pm 4$ months. This deciduous dentition remains intact until the child is about 6 years of age. At about that time the first succedaneous (permanent) teeth begin to emerge into the mouth. The emergence of these teeth marks the beginning of the mixed dentition period in which a mixture of deciduous and succedaneous teeth is present. This transition period lasts from about 6 to 12 years of age and ends when all the deciduous teeth have been shed. At that time the permanent dentition period begins. Thus, the transition from the primary dentition to the permanent dentition begins with the emergence of the rst permanent molars, shedding of the deciduous incisors, and emergence of the permanent incisors.

The mixed dentition period is often a difficult time for the young child due to many reasons including habits, missing teeth, teeth of dierent colors and hues, crowding of the teeth, and malposed teeth. After the shedding of the deciduous canines and molars, emergence of the permanent canines and premolars, and emergence of the second permanent molars, the permanent dentition is completed (including the roots) at about 14 to 15 years of age, except for the third molars, which are completed at 18 to 25 years of age. In effect, the duration of the permanent dentition period is $12+$ years.

The completed permanent dentition consists of 32 teeth if none are congenitally missing, which may be the case (see Figure 10). Teeth of humans are small, hard, calcied and whitish structures found in the mouth. The visible part of these teeth is called the crown, while the invisible part, which is embedded in the jaw bone is referred to as the tooth root. The molars, and the premolars of the upper jaw, usually have two, three or four dierent roots and are called multi-rooted teeth. 


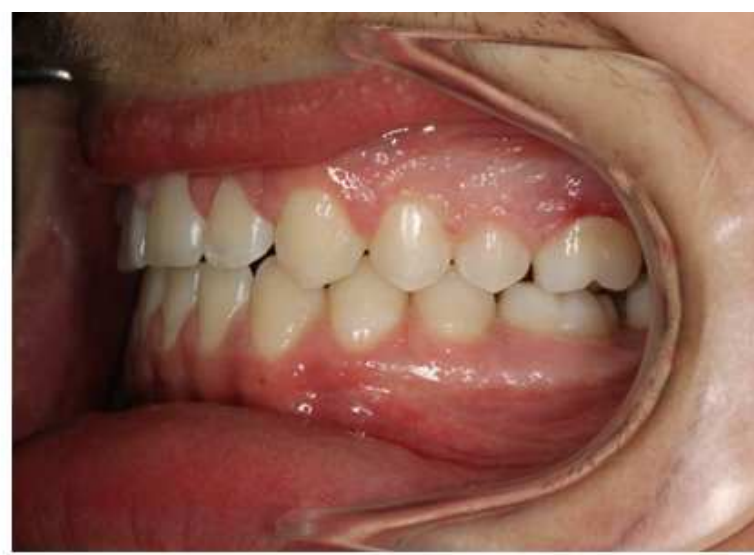

(a)

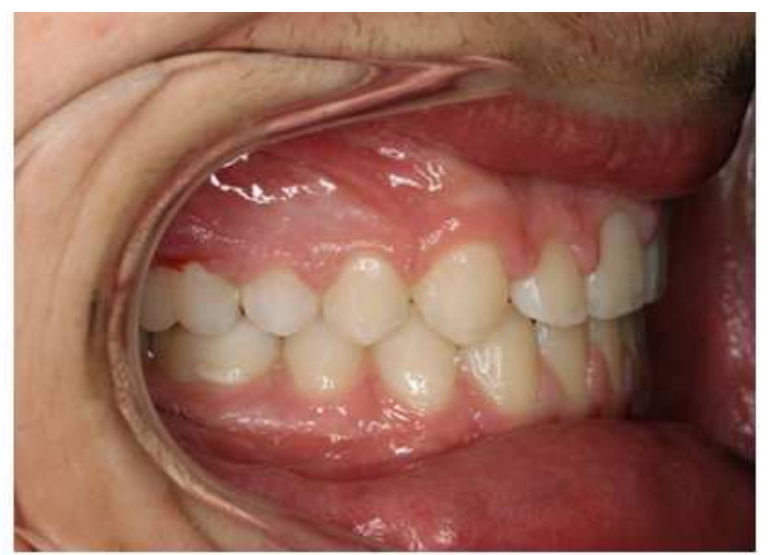

(b)

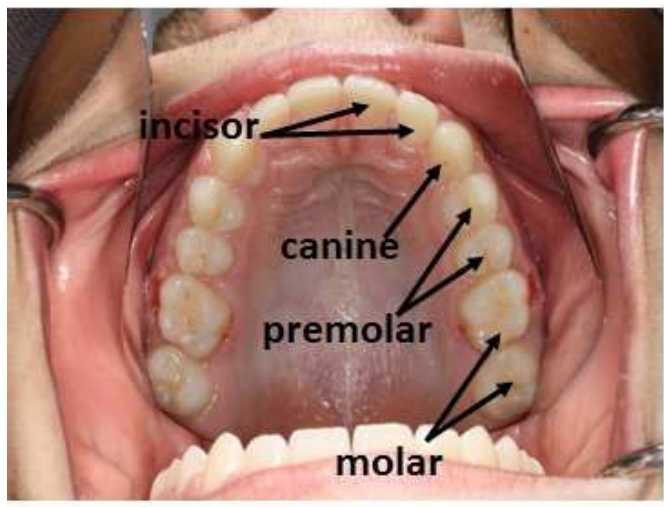

(c)

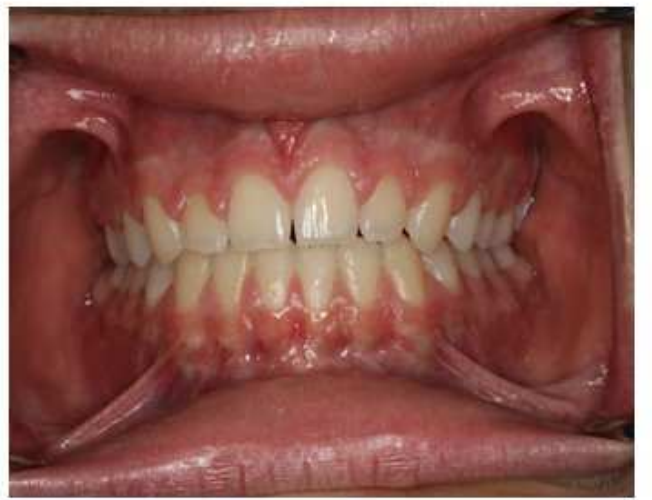

(d)

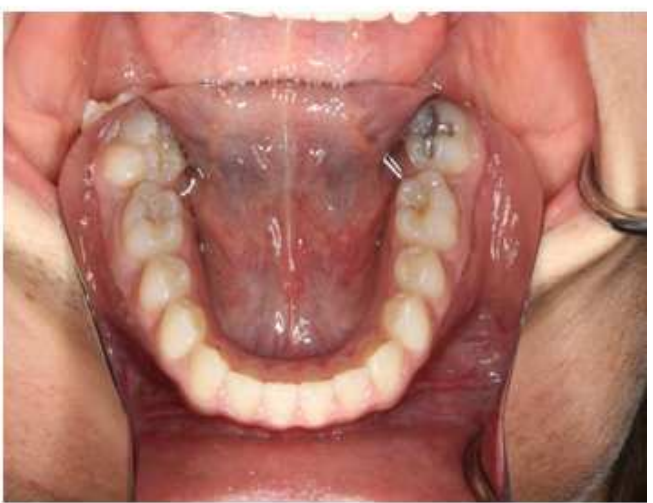

(e)

FIGURE 10: Anatomy of the tooth. (a) intraoral left. (b) intraoral right. (c) occlusal upper: schematic overview of the distribution of the different teeth in an adult mouth. (d) intraoral center. (e) occlusal lower. 


\section{SURFACES AND RIDGES}

The crowns of the incisors and canines have four surfaces and a ridge, where as the crowns of the premolars and molars have ve surfaces. The surfaces are named according to their anatomical positions and uses (see Figure 10). In the incisors and canines, the surfaces toward the lips are called labial surfaces; in the premolars and molars, surfaces facing the cheek are the buccal surfaces. Both the labial and buccal surfaces together are referred to as facial surfaces. All surfaces facing toward the tongue are called lingual surfaces. The surfaces of the premolars and molars that come in contact (occlusion) with those in the opposite jaw during the act of closure are called occlusal surfaces. These are called incisal surfaces with respect to incisors and canines.

2. The periodontal ligament

Figure $11^{1}$ shown the periodontal ligament (PDL) that supporting structure of the tooth, attaching it to the alveolar bone. The PDL in the humans has an average width of $0.25 \mathrm{~mm}$ [23]. The PDL consists of principal fibres stretching across the width of the ligament, which are embedded as Sharpeys fibres in the bone and the cementum.

\section{E. Reconstruction of the Human Jaw}

In 1997, Ahmed et al. [24] introduced the first optical approach to construct the human jaw from a sequence of images taken by intraoral camera. The SFS solution provides an estimate of the shape for a certain light direction (perspective projection); this estimate is non-metric, and real world surfaces (e.g., the human jaw) are not Lambertian. In 1999, Yamany et al. [25] introduced a modification to the SFS problem by incorporating sparse

\footnotetext{
${ }^{1}$ http://www.highlands.edu/academics/divisions/scipe/biology/faculty/harnden/2122/images/toothanatomy.jpg
} 


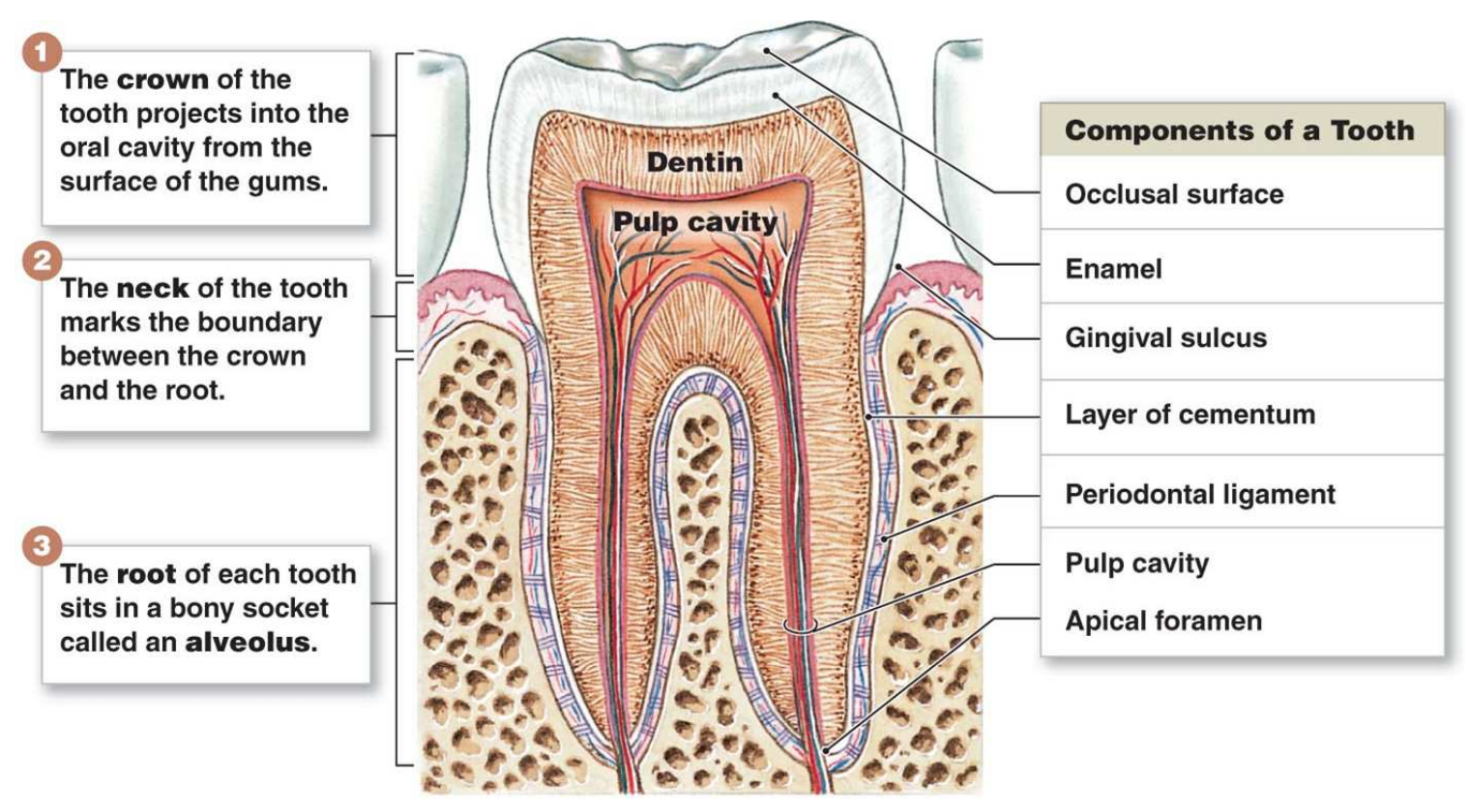

FIGURE 11: Schematic overview of the anatomy of the tooth and its surrounding structure: the crown is covered with enamel, root, cusp, dentin, gum, cementum, periodontal ligament, alveolar bone and apex.

3D points, obtained by a touch probe, into the camera calibration model, and used a filter to reduce the jaw specularity, in order to make the Lambertian assumption adequate. In 2000, Yamany et al. [18] introduced the first computer vision system for 3D jaw reconstruction based on an intraoral camera mounted on a six-segment coordinate measuring unit. Since then, various modifications to the imaging process and software optimization were carried out, which enhanced the capabilities of the Computer Vision and Image Processing (CVIP Lab) Dental Station.

The accuracy obtained by the CVIP Lab Dental Station (as shown in Figure 12) was in the millimeter range, yet it has the following drawbacks: i) The camera required a pre-calibration and needed to be hooked to the coordinate measuring arm, in order to reference the image sequence used in the reconstruction to a common 3D referencing system. ii) The data acquisition was awkward, requiring cooperation of the object during data gathering which may last up to five minutes. iii) The overall accuracy of the resulting 3D 
model was not suitable for various envisioned dental practices. Two obvious enhancements were essential to make the Dental Station more practical: Making the dental probe flexible; and improving the 3D model building algorithm from sequence of images. Major efforts have been undertaken to address these two shortcomings. Focused on generalization of the SFS solution to various object characteristics as stated above [12,26], and deployed newer cameras with various light sources and motion sensors. From algorithmic point of view we introduced a new stitching algorithm [20], which enabled registration of the image segments covering the human jaw, then applying SFS vs. applying SFS on individual segments and then register the results. Equally significant is that we examined the roughness of the jaw material using profiler microscopes available at the University of Louisville MEMS facility, in order to calibrate the albedo parameters in the SFS using realistic measures.

\section{F. Contributions of this Dissertation}

This dissertation involves theoretical developments, system design and integration, as well as practical evaluation by dental professionals. In the context of tooth reconstruction from intraoral images, the SFS technique offers several advantages. It provides more detailed and accurate representations about the shape of the tooth crowns [18]. It requires only one camera position within the cramped confines of the mouth. Cost-wise, it is cheaper because it simply requires a single camera and light source. Summarizing the main contributions of this work:

- A robust data acquisition system has been proposed that acquires calibrated images for the jaw by an intraoral camera and controlled lighting mechanism.

- This work designed and developed novel algorithms for 3D surface reconstruction by SFS aspect for human teeth based on a single image. A non-Lambertian SFS algorithm under perspective projection is proposed, which benefits from camera calibration parameters. Take into account the attenuation of illumination due to near- 


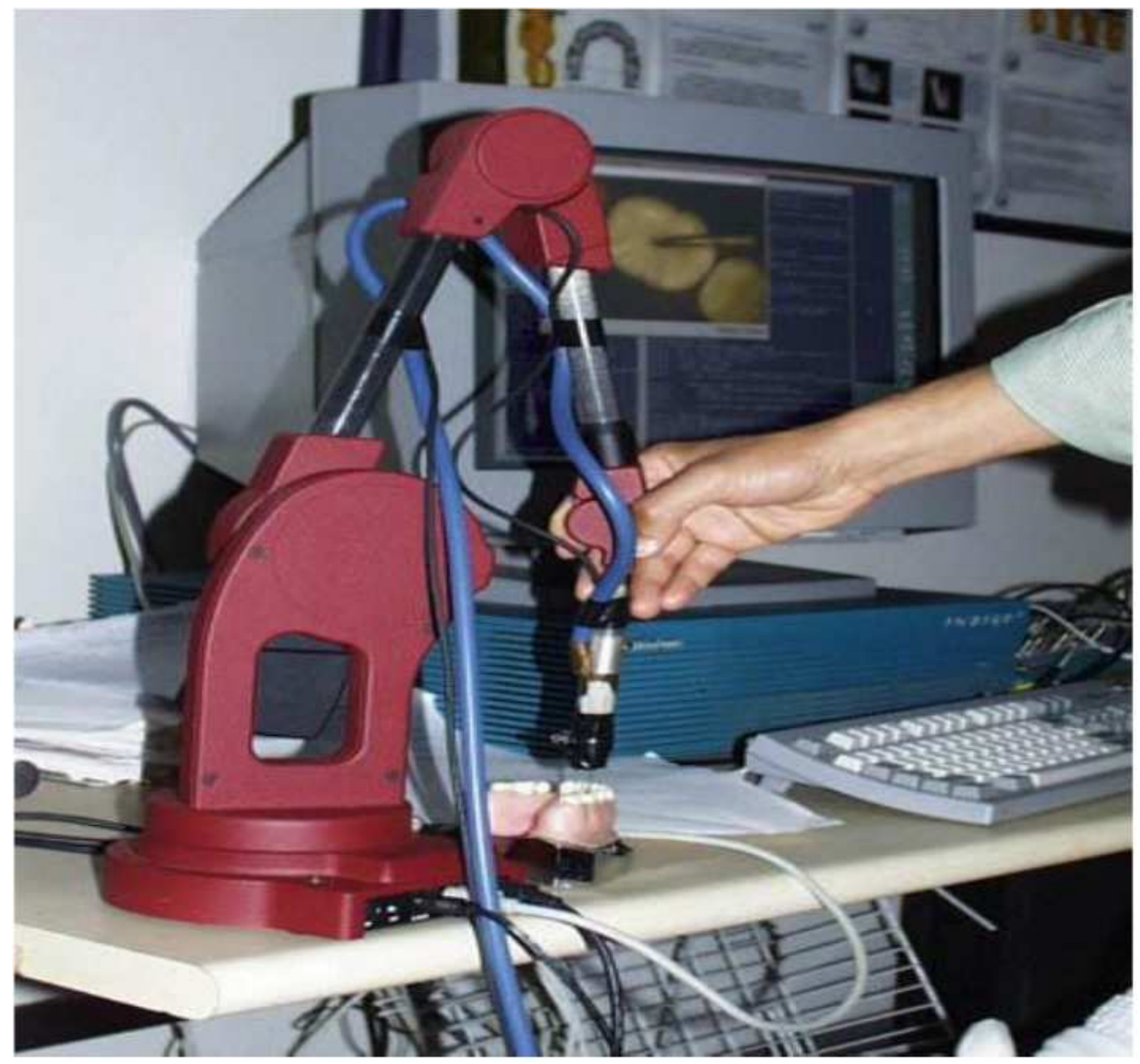

FIGURE 12: Scientific prototype of the dental probe at the CVIP Lab (2000-2004). A CCD camera attached to a coordinate measuring arm captures the video images used in the jaw reconstruction. 
field imaging. The surface reflectance is modeled using the Oren-Nayar-Wolff model which accounts for the retro-reflection case.

- A novel method to incorporate the 3D shape priors in SFS formulation was proposed. This strategy is motivated by the fact that humans rely on strong prior information about the 3D world around us in order to perceive 3D shape information.

- 2D-PCA is used to build the shape priors instead of the conventional PCA. The 2DPCA offers two important advantages: It is easier to evaluate the covariance matrix accurately since its size is much smaller. In addition, less time is required to determine the corresponding eigenvectors. Second, the modified Oren-Nayar-Wolff reflectance model is presumed in place of the Oren-Nayar model, where teeth surface is rough and wet, giving rise to Fresnel reflection due to different refractive indices of the saliva and the tooth material.

- The tooth surface roughness is physically measured using an optical surface profiler and stylus arm.

- Developing techniques for stitching 3D surface patches reconstructed from different views of the jaw surface were proposed. Calibration parameters have been computed adaptively while the camera is moving using the sensors to generate calibrated images and meaningful surfaces. Solving this problem touches on all aspects for computer vision and image processing.

- A model-based SFS approach is proposed which allows for the construction of plausible human jaw models in vivo, without ionizing radiation, using fewer sample points in order to reduce the cost and intrusiveness of acquiring models of patients teeth/jaws over time.

- Establish a dental database and a procedure to augment it by researchers in the US and worldwide, for enhancing dental research and practice on the human teeth/jaw. 
- The possibility of calculating the initial stresses and strains in the PDL is calaculated analytically and using FEA.

\section{G. Organization of the Dissertation}

The dissertation is given in nine chapters. A summary of each one is presented which includes the problem, contribution, and experimental results.

\section{Chapter I}

This chapter includes the definition of SFS, 3D reconstruction from sequence of images, the basic of the camera calibration, teeth anatomy and the related work of the reconstruction of the human jaw. Also it has in brief, the contributions to solve the problem.

\section{Chapter II}

This chapter gives an brief introduction to surface reconstruction by geometric computer vision and image irradiance equations.

\section{Chapter III}

At the University of Louisville Dental school there exists enormous number of molds/teeth and patient records. The first steps to arrange these molds/teeth are taken into subjects categorized with respect to gender, age and ethnicity. This database is very important in 1) a model-based SFS approach which allows for the construction of plausible human jaw models in vivo, without ionizing radiation, using fewer sample points in order 
to reduce the cost and intrusiveness of acquiring models of patients jaws/teeth over time. 2) using for FEA. Also, it has an introduction to orthodontic tooth movement using analytical method.

\section{Chapter IV}

This chapter describes a new method of 3D surface reconstruction aspect for human teeth based on a single image, which provides more realistic formulation of the SFS problem by considering the image formation components: the camera, the light source, and the surface reflectance. A non-Lambertian SFS algorithm is proposed under perspective projection which benefits from camera calibration parameters. The attenuation of illumination due to near-field imaging is taken into account. The surface reflectance is modeled using the Oren-Nayar-Wolff model which accounts for the retro-reflection case.

\section{Chapter V}

This chapter proposes a new variational formulation that relates an evolving surface model with image information, taking into consideration that the image is taken by a perspective camera with known parameters. A new energy functional is formulated to incorporate brightness, smoothness, and integrability constraints. Furthermore, an automatic approach for 3D surface reconstruction of the human jaw using calibrated images with the help of the ASIFT features matching is presented.

\section{Chapter VI}

This chapter developers a new approach for realistic 3D reconstruction of the human teeth using shape from shading with statistical shape priors gathered from an ensemble of 
scanned human teeth, in order to improve the quality of SFS as applied to jaw surface.

\section{Chapter VII}

The approached developed in this chapter reconstructs the teeth from single image shading with 2D-PCA shape priors that have a more sophisticated reflectance model. The Oren-Nayar-Wolff model was used for modeling the surface reflectance.

\section{Chapter VIII}

In this chapter, an accurate three-dimensional CAD model is proposed. 3D stress and displacements of different teeth type are successfully carried out using FEA.

\section{Chapter IX}

This chapter concludes the dissertation with insights to extensions to be handled during thesis work. 


\section{CHAPTER II}

\section{SURFACE RECONSTRUCTION BY GEOMETRIC COMPUTER VISION}

Simply stated, the surface reconstruction problem is the following: given a sequence of calibrated and referenced 2D images of an object, construct a 3D representation of that object. If the 2D image sequence covers the entire object (i.e., 360 degrees) then the 3D reconstruction is expected to be a full 3D surface representation. This problem has been studied in the past 40 years in what is known in computer vision as the "shape from X" problem. Where $\mathrm{X}$ represents the various cues employed in going from $2 \mathrm{D}$ to $3 \mathrm{D}$, which includes stereo (e.g. [27-31]), texture (e.g. [32,33], motion (e.g. [34-37]), and shading (e.g. [38-46]). Other approaches in geometric computer vision that are more recent are the voxel-based approaches, e.g. voxel coloring [47], space carving [48], and generalized voxel coloring [49]. These methods extract the 3D information of an object by removing (carving) the volume elements (voxels) in the initial 3D volume that are invisible and photoinconsistent in all/subset of the sequence of images. A plethora of algorithms exists in the literature for reconstruction and recognition from sequence of images. Even though the geometry of the reconstruction has been well-developed through the foundational work of a number of investigators (e.g., [38], Grimson [50], Faugeras [51], Hartely and Zisserman [52]), the accuracy of the reconstructions is application oriented and there is no existing geometric reconstruction mechanism that fits all needs. Considerable work still remains in order to achieve automatic 3D surface reconstruction from video.

Difficulties in video reconstruction come, in part, from the fact that the assumptions for typical geometrical reconstruction are hardly valid. For example, assumptions in stereo (e.g., presence of distinct features of the objects in the right and left images), in SFS (e.g., 
the Labmertian assumption that considers the surface in the field of view of the camera to be mat), in space carving (e.g., the photo consistency) are rarely valid in practice. Other difficulties result from surfaces that are glossy, transparent, occluding, and mutually illuminating. In addition, errors result from the data acquisition sensors (e.g., camera calibration errors due to motion, lens distortion, etc.). Despite these problems, video-based technology is extremely powerful and popular in every facet of life. The system uses this technology for creating a 3D surface model of the object from sequence of images.

\section{A. Shape from Shading (SFS)}

Among the tools used in shape extraction from single view is the SFS technique. SFS has been primarily studied by Horn [38] and it extracts the depth (hidden information) from the image formation process that relates source irradiance to image gray level intensity. There have been various developments in the SFS algorithm (e.g., [53-59]). The most important information for reconstructing an accurate $3 \mathrm{D}$ visible surface, which is missing in SFS, is the metric measurement. SFS also suffers from the discontinuities due to highly textured surfaces and different albedo [38]. In [18], they introduced an algorithm that integrates the dense depth map obtained from SFS with sparse depth measurements obtained from a coordinate measurement machine $(\mathrm{CMM})$ for the reconstruction of $3 \mathrm{D}$ surfaces. This algorithm provides two advantages: it removes the ambiguity of the 3D visible surface discontinuities produced by SFS, and it complements for the missing metric information. The integration process includes the following stages. First, calculated the error difference in the available depth measurements between the two sets of sensory data. Then, approximated a surface that fits this error difference. Finally, the approximated surface is used to correct the shape from shading. The reconstruction involved several sequential steps: Camera calibration, imaging and landmark/ground truth collection, SFS extraction, merging with ground truth points using Neural Network(NN), and obtaining the 3D surface. Extensions to the previous algorithm [18] include flexible and more accurate 
image data collection, quantification of motion artifacts, reduction in algorithmic time, and studying the robustness of the overall procedure to ensure accuracy and repeatability.

Shape-from-Shading is a classic and challenging problem in computer vision. It uses the brightness variation in a single image to compute the $3 \mathrm{D}$ shape of a surface. The goal of SFS is to solve the image irradiance equation, which robustly relates the reflectance map to image intensity. However, the task appears to be nontrivial. Consequently, most work in this relies on assumptions which simplify the irradiance equation. Of particular importance is the common assumption that scene points are projected orthographically during the photographic process.

Many works in the field of SFS have followed the seminal works of Horn [60-62] who initiated the subject in the 1970s, and assumed orthographic projection. Horn's book [63] reviews the early work on SFS (until 1989). Zhang et al. [8] survey and classify some of the works from the '1990s and compare the performance of six of them (namely, minimization approaches: [64], [65]; propagation approaches: [66]; local approaches: [67]; linear approaches: [68], [69]). Kimmel and Bruckstein [70] classify image extrema and two kinds of saddle points and use these topological properties of the surface in a global SFS algorithm. Zhao and Chellappa [71] use symmetric SFS to develop a face recognition system which is illumination insensitive. They show that the symmetric SFS algorithm has a unique solution. Kimmel and Sethian [72] proposed the Fast Marching method as an optimal algorithm for surface reconstruction. Their reconstructed surface is a viscosity solution of an Eikonal equation for the vertical light source case. Sethian [73] provides deep insight into Level Set and Fast Marching methods. Prados et al. [74] base their approach on the viscosity solution of a Hamilton-Jacobi equation. They extend existing proofs of existence and uniqueness to the general light source case and prove the convergence of their numerical scheme. Many more orthographic algorithms were suggested in the literature, but only a few can be described herein.

SFS algorithms can be categorized into four main groups [69]: minimization ap- 
proaches, propagation approaches, local approaches, and linear approaches. Minimization approaches obtain the solution by minimizing an energy functional. Propagation approaches propagate the shape information from a set of surface points (e.g., singular points) to the whole image. Local approaches extract shape based on the assumption of surface type. Linear approaches compute the solution based on the linearization of the reflectance map. To solve the SFS problem under more comprehensive modeling conditions, we need very powerful mathematical tools. Basically, we can choose between propagation approaches or energy minimization approaches since the applicability of the local approaches is limited, and the reasonability of the linear approximation of the reflectance map is questionable [75].

One of the earlier and most important works in the minimization approaches, which reconstructs the surface gradients, was by Ikeuchi and Horn [76]. Each surface point has two unknowns for the surface gradient and each pixel in the image provides one intensity value, which yields to we having an under-determined system. To overcome this, they introduced two constraints: the brightness constraint and the smoothness constraint. The brightness constraint requires that the reconstructed shape produces the same brightness as the input image at each surface point, while the smoothness constraint ensures a smooth surface reconstruction. The shape was computed by minimizing an energy function which consists of the above two constraints. To ensure a correct convergence, the shape at the occluding boundary was given for the initialization. Since the gradient at the occluding boundary has at least one infinite component, stereographic projection was used to transform the error function to a different space. Also using these two constraints, Brooks and Horn [77] minimized the same energy function in terms of the surface normal. Frankot and Chellappa [78] enforced integrability in Brooks and Horn's algorithm in order to recover integrable surfaces. Surface slope estimates from the iterative scheme were expressed in terms of a linear combination of a finite set of orthogonal Fourier basis functions. The enforcement of integrability was done by projecting the nonintegrable surface slope estimates onto the nearest integrable surface slopes. This projection was fulfilled by finding the clos- 
est set of coefficients which satisfy integrability in the linear combination. Their results showed improvements in both accuracy and efficiency over Brooks and Horn's algorithm [77]. Later, Horn also [38] replaced the smoothness constraint in his approach with an integrability constraint. The major problem with Horn's method is its slow convergence.

\section{B. Geometric Stereo}

The availability of a sequence of calibrated images enables the application of other reconstruction methods (e.g., stereo and space carving). The stereo approach matches information present in the left and right images (any two images in the referenced sequence that share common information about the scene) in order to extract the depth (disparity or third dimension) from the two images. If the sequence of images cover the entire object, the pairwise reconstructions can be merged together to form a full 3D model. Pairwise images from the sequence (obtained by a single camera) that are related may be used as a "stereo-pair" to extract the depth.

In the general stereo configuration, the image planes are not coplanar which makes the correspondence problem more difficult than the simple stereo configuration. If no constraints are applied to this configuration, then it is necessary to search for the point $\mathrm{x}_{r}$ in the entire right image to be matched with $\mathrm{x}_{l}$ (note: the right and left images may be thought of just as two related images from the sequence). The search space can be limited to a general line in the right image if the epipolar constraint [30] is applied. Furthermore, image rectification algorithms (e.g., [79]) can be used to parallelize and align the epipolar lines to the $\mathrm{x}$-axis. This rectification step reduces the problem to a simple stereo configuration problem.

In general, the stereo approaches operate either by the edge-feature matching or the area-based matching. Feature-based stereo approaches are useful because they describe the important geometry of the object. However, the major problem of most of these approaches 
is the low output density. A dynamic programming [30] approach matches features to get dense reconstruction, but it can only be applied on a scan line by scan line basis. Boykov, et al. [80] and Roy and Cox [28] solved the inter-scan line problem using graph cuts. In contrast to the feature-based stereo, the area-based stereo provides dense reconstructions. Okutumi and Kanade [29] used a variable size correlation window to generate dense depth maps. However, area-based usually fails when applied to surfaces of large textureless areas. Another challenging problem for stereo approaches is the occlusion problem, where scene elements appear in one image but they are occluded in the other image.

With respect to smooth objects, the major sources of errors are the lack of specific features on the target when viewed in the front or the back; only the upper views of the target can provide some feature for matching. In addition, various sources of inaccuracies in depth measurements may result due to effects of motion, lightning and the occlusion due to the nature of the object.

\section{Space Carving}

Space carving [48] attempts to produce the maximal 3D shape that is consistent with all the images. Space carving starts with an initial volume V that includes the object to be reconstructed. This $3 \mathrm{D}$ space is then discretized into a finite set of voxels $\mathrm{v}_{1}, \mathrm{v}_{2}$, $\ldots, \mathrm{V}_{n}$. The idea is to successively carve (remove) some voxels until the final 3D shape, $\mathrm{V}^{*}$, agrees with all the input images. Each voxel on the surface of the volume, i.e., in Vis(V), is projected back to the different images using their respective projection matrices. A voxel is carved or not is based on color-consistency. The Lambertian model for the surface of the object is assumed. Under this model, light reflected from a single point on the surface of the object has the same intensity in all directions. Therefore, for a voxel to belong to the surface of the object, it must have the same color intensity, within some tolerance to allow for some light variations and some calibration inaccuracy, for all its projections to 
the different images provided. Voxels that are inconsistent with a single color are viewed as free space in which different light rays intersect. By removing all color-inconsistent voxels, we are able to approximate a maximal photo-consistent shape that is defined by all the input images.

\section{Integration of Reconstruction Methods}

No single approach in geometric computer vision fits all applications. Integration/fusion of several approaches has been attempted in order to improve the reconstruction results. For example, Fua and Leclerc [37] developed an approach for reconstructing both the shape and reflectance properties of surfaces from multiple images. The method begins with an initial estimate of surface shape provided, for example, by triangulating the result of conventional stereo. The surface shape and reflectance properties are then iteratively adjusted to minimize an objective function that combines information from multiple input images. The objective function is a weighted sum of stereo, shading, and smoothness components, where the weight varies over the surface. Lange [38] proposed another approach for integration of SFS and stereo in which he corrects for error propagation from stereo vision to SFS, when only the initial and border conditions are used for the cooperation, by the introduction of simultaneous constraints from both modules on all image points. Samaras et. al [39] presented a multiview method for the computation of object shape and reflectance characteristics based on the integration of SFS and stereo, for non constant albedo and non-uniformly Lambertian surfaces. First stereo fitting on the input stereo pairs or image sequences is performed. Based on the stereo result, the albedo map can be automatically segmented (which is taken to be piece-wise constant) using a minimum description length (MDL) based metric, to identify areas suitable for SFS (typically smooth textureless areas) and to derive illumination information. The shape and the illumination parameter estimates are refined using a deformable model SFS algorithm, which iterates between computing shape and illumination parameters. Fassold et. al [40] have 
introduced an algorithmic framework for the refinement of sparse 3D models using shape from shading. Starting from an initial model obtained by shape from stereo, they use a global optimization scheme in order to refine the surface. The constraints used are based on the shading in the image, the initial 3D points obtained by stereo and the smoothness of the surface. In contrast to other approaches that assume that the photometric properties of the scene are known they iteratively update the light source direction and several parameters of the reflectance map.

\section{E. Image Formation Model}

How pixel brightness in the image is related to the physical world is the answer of two questions; (1) where some point in 3D will appear in the 2D image, and (2) how bright this image point will be. The former question is related to the camera/viewer properties, i.e. geometric image formation, while the latter one is governed by the surface physical and reflectance properties as well as the illumination conditions, i.e. photometric image formation [81].

Assuming the camera extrinsic parameters are solved for w.r.t. a predetermined world coordinate frame, consider a viewer/camera-centered coordinate system with the camera lens located at the origin $O$, i.e. optical center, where the optical axis coincides with the $z$-axis. Since the image is formed in the near field, the geometric imaging process can be modeled by the pinhole (perspective) projection where the image/retinal plane is located at a distance governed by the camera's focal length $f$. Let $\mathbf{M}=(X, Y, Z)^{T}$ denote a surface point perceived in the camera frame, where $Z=Z(X, Y)$ represents the depth value of the surface point $\mathbf{M}$ whose projection is denoted by $\mathbf{m}=(x, y)^{T}$ on the image plane.

\section{F. Image Irradiance}




\section{Lambertian Reflection Model}

The SFS problem consists of recovering the 3D-shape of a surface through the analysis of the brightness variation in a single image. In general, the brightness of a surface patch depends on its orientation relative to both the light source and the viewer. The image irradiance equation models this relationship as

$$
E(\mathbf{x})=R(\hat{\boldsymbol{n}}(\mathbf{x}))
$$

where $E(\mathbf{x})$ is the image irradiance at the point $\mathbf{x}$ and $R($.$) is the radiance of a surface$ patch with unit normal $\hat{\boldsymbol{n}}(\mathbf{x})$.

For simplification purposes, most of the algorithms in SFS literature assumed that the surface has a Lambertian reflectance [17], i.e., the surface reflects the light equally in all directions. In this case the reflectance map is the cosine of the angle between the unit vector $\hat{\boldsymbol{s}}$ in the light direction and the normal vector $\hat{\boldsymbol{n}}$ :

$$
E(\mathbf{x})=R=\cos \angle(\hat{\boldsymbol{s}}, \hat{\boldsymbol{n}})=\hat{\boldsymbol{s}} \cdot \hat{\boldsymbol{n}},
$$

which leads to the first PDE studied in the SFS literature:

$$
I(\mathbf{x}) \sqrt{1+|\nabla u(\mathbf{x})|^{2}}+\hat{\boldsymbol{s}} \cdot(\nabla u(\mathbf{x}),-1)=0,
$$

where $u(\mathbf{x})$ is the surface height at point $\mathbf{x}=(x, y)$ above some reference plane. The camera is assumed here to perform orthographic projection, which is also a simplification of the real perspective projection done by a camera. Note that the image irradiance $E$ has been replaced by the measured image gray value $I$ by assuming a linear relationship between them and dropping the scaling factor. Under real world circumstances the surface materials are not Lambertian, and in many cases the camera and the light are not far away from the object. 


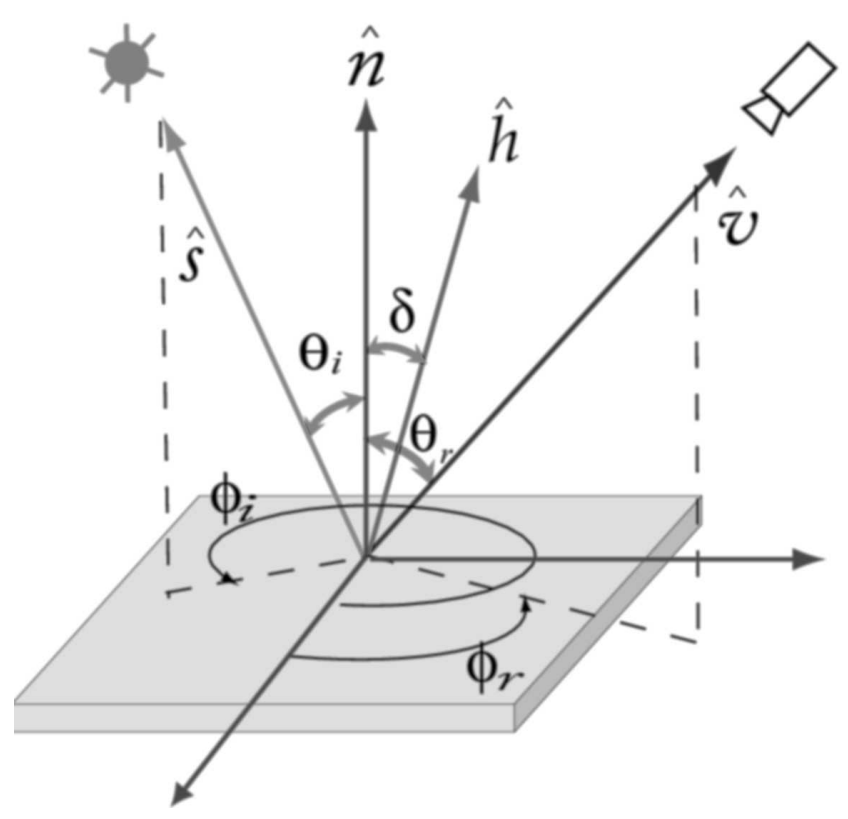

FIGURE 13: Definitions of reflection parameters and angles.

\section{Oren-Nayar Diffuse Reflection Model}

The Oren-Nayar diffuse reflection model has been introduced for rough surfaces [15], which can be seen as a generalization of Lambertian reflectance for rough diffuse surfaces. The roughness of the surface is specified using a Gaussian distribution for the orientations of the approximating surface facets. Using the geometry illustrated in Figure 13, given the radiance of the incoming light $L_{i}$, the radiance of the reflected light $L_{r}$, the Oren-Nayar model can be given by:

$$
\begin{gathered}
L_{r}=\frac{\rho}{\pi} L_{i} \cos \theta_{i}\left(A+B \sin \alpha \tan \beta \max \left[0, \cos \left(\phi_{r}-\phi_{i}\right)\right]\right) \\
\text { where } A=1-0.5 \frac{\sigma^{2}}{\sigma^{2}+0.33}, B=0.45 \frac{\sigma^{2}}{\sigma^{2}+0.09}
\end{gathered}
$$

The parameter $\sigma$ denotes the standard deviation of the orientations Gaussian distribution, and it is used as a measure of the surface roughness, $\alpha=\max \left\{\theta_{r}, \theta_{i}\right\}, \beta=\min \left\{\theta_{r}, \theta_{i}\right\}$ and $\rho$ is the diffuse albedo.

Assume the camera is modelled with a perspective projection. According to the 


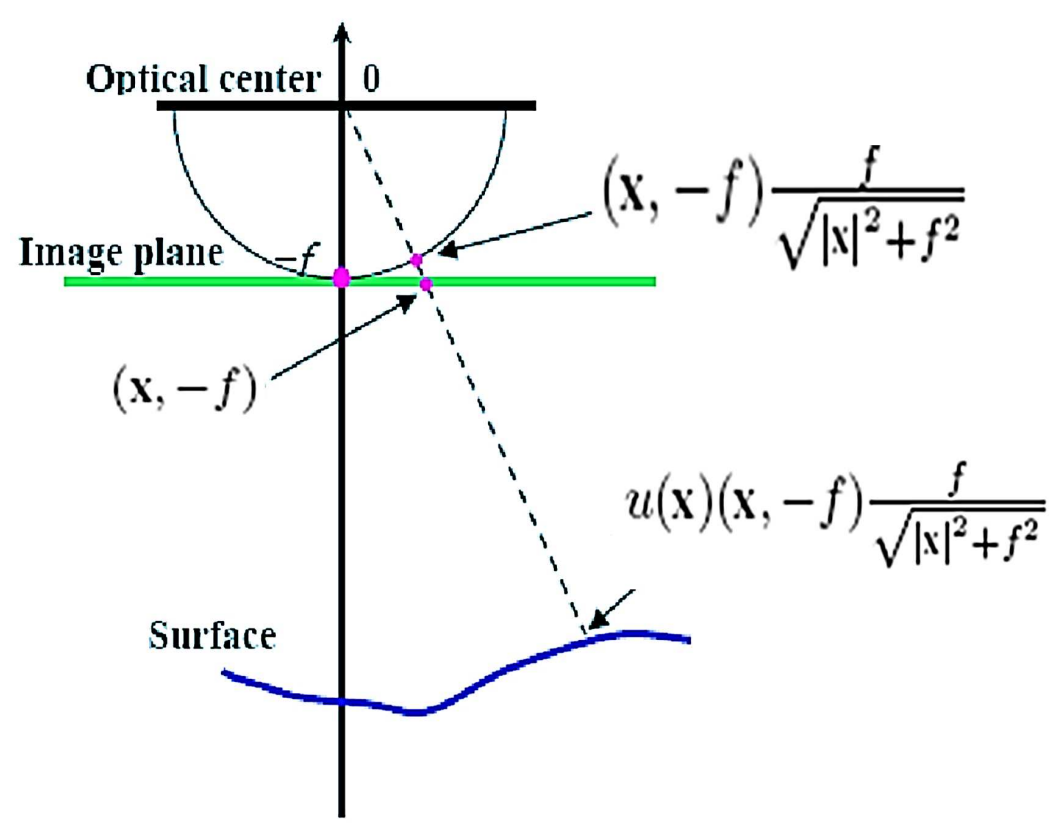

FIGURE 14: Perspective camera model for SFS.

proposed data acquisition setup, the light source is located at the optical center. Then, $\theta_{r}=$ $\theta_{i}=\alpha=\beta \doteq \theta$. The surface is represented by [9,22] (see Figure 14): $\mathcal{S}=\{S(\mathbf{x}) / \mathbf{x} \in \Omega\}$, where $S(\mathbf{x})=\frac{f u(\mathbf{x})}{\sqrt{|\mathbf{x}|^{2}+f^{2}}}(\mathbf{x},-f)$, with $f$ being the camera's focal length. The surface normal at any point is given by $\hat{\boldsymbol{n}}(\mathbf{x})=\left[f \nabla u(\mathbf{x})-\frac{f u(\mathbf{x})}{|\mathbf{x}|^{2}+f^{2}} \mathbf{x}, \nabla u(\mathbf{x}) \cdot \mathbf{x}+\frac{f u(\mathbf{x})}{|\mathbf{x}|^{2}+f^{2}} f\right]$.

The light source direction equals $\hat{\boldsymbol{s}}=\frac{1}{\sqrt{|\mathbf{x}|^{2}+f^{2}}}(-\mathbf{x}, f)$. Furthermore, the attenuation of the illumination is taken into account due to the distance $r$ between the light source and the surface. This helps resolve the convex/concave ambiguity from which traditional SFS algorithms suffer when this attenuation phenomenon is ignored $[9,22]$. This distance is given by $r=f u(\mathbf{x})$. As a result, (11) eventually becomes

$$
L_{r}(\theta ; \sigma)=\left(\frac{\rho}{\pi} L_{i}\right) \frac{A \cos (\theta)+B \sin ^{2}(\theta)}{r^{2}}
$$




\section{CHAPTER III}

\section{DENTAL DATABASE CONSTRUCTION}

\section{A. Introduction}

Many dental applications such as endodontic procedures, treatment of malocclusion problems and treatment simulations require an accurate 3D representation of the teeth and the jaw. Using 3D CAD models has great advantages over conventional physical solid models, as solid models require big physical space for storage, adding financial and logistic burdens which is not the case of CAD models that are stored electronically [82]. This chapter proposes to collect resources for the jaw project in a common repository for dissemination among researchers elsewhere. At the University of Louisville Dental school there exists enormous number of molds and patient records. The first steps were arraigning these molds into subjects categorized with respect to gender, age and ethnicity(see Table 1). Using the Conebeam CT scanner, these molds were scanned and the image processing tools were used to create a mesh per mold as shown in Figure 17 and Figure 18. The data used to generate the Statistical Shape from Shading (SSFS) results in [83] were obtained from this data. In this dissertation, the data (images and corresponding 3D mesh from the molds) were annotated and are available to colleagues elsewhere.

\section{B. Tooth Database Construction}

In [84] serves a step towards complimenting a surface model with root information 
obtained from X-rays based on 3D teeth library. Yet the database was limited and validations were mostly done on manufactured jaws. In this dissertation, further investigate the fusion process of the whole human jaw to add a close proximity of the root information to the proposed 3D surface model.

Several real invitro teeth of the same type (maxillary deciduous, maxillary molars, mandibular molars, and mandibular third molars) from adult subjects with different races, gender and ages are fixed over wax. A Cone-beam CT (KODAK 9000 3D Extraoral) scanner at a resolution of $0.2 \mathrm{~mm}$ is then used to scan the wax and teeth. ExpectationMaximization (EM) algorithm for segmentation is used. Afterwards, the 3D surfaces for each tooth type are rigidly aligned to remove any variations in shape due to pose differences. The first surface of the training set is used as the reference to which the remaining surfaces are aligned. The alignment is carried out using an iterative closest point (ICPbased) rigid registration algorithm [85] using the Hausdorff distance between corresponding points. Figure 15 show samples of the acquired CT scans.

Currently, the database having 224 teeth with the following statistics: anterior (20), mandibular 3rd molar (119), maxillary deciduous (27), maxillary Molars (38) and premolars (20). They are automatically preprocessed to generate triangular meshes, see Figure 15.

\section{Iterative Closest Point (ICP)}

ICP is an algorithm used to minimize the difference between two clouds of points [8688]. ICP is often used to reconstruct $2 \mathrm{D}$ or $3 \mathrm{D}$ surfaces from different scans, to localize robots and achieve optimal path planning (especially when wheel odometry is unreliable due to slippery terrain), to co-register bone models, etc. In the algorithm, one point cloud, the reference, or target, is kept fixed, while the other one, the source, is transformed to best

match the reference. The algorithm iteratively revises the transformation (combination of translation and rotation) needed to minimize the distance from the source to the reference 

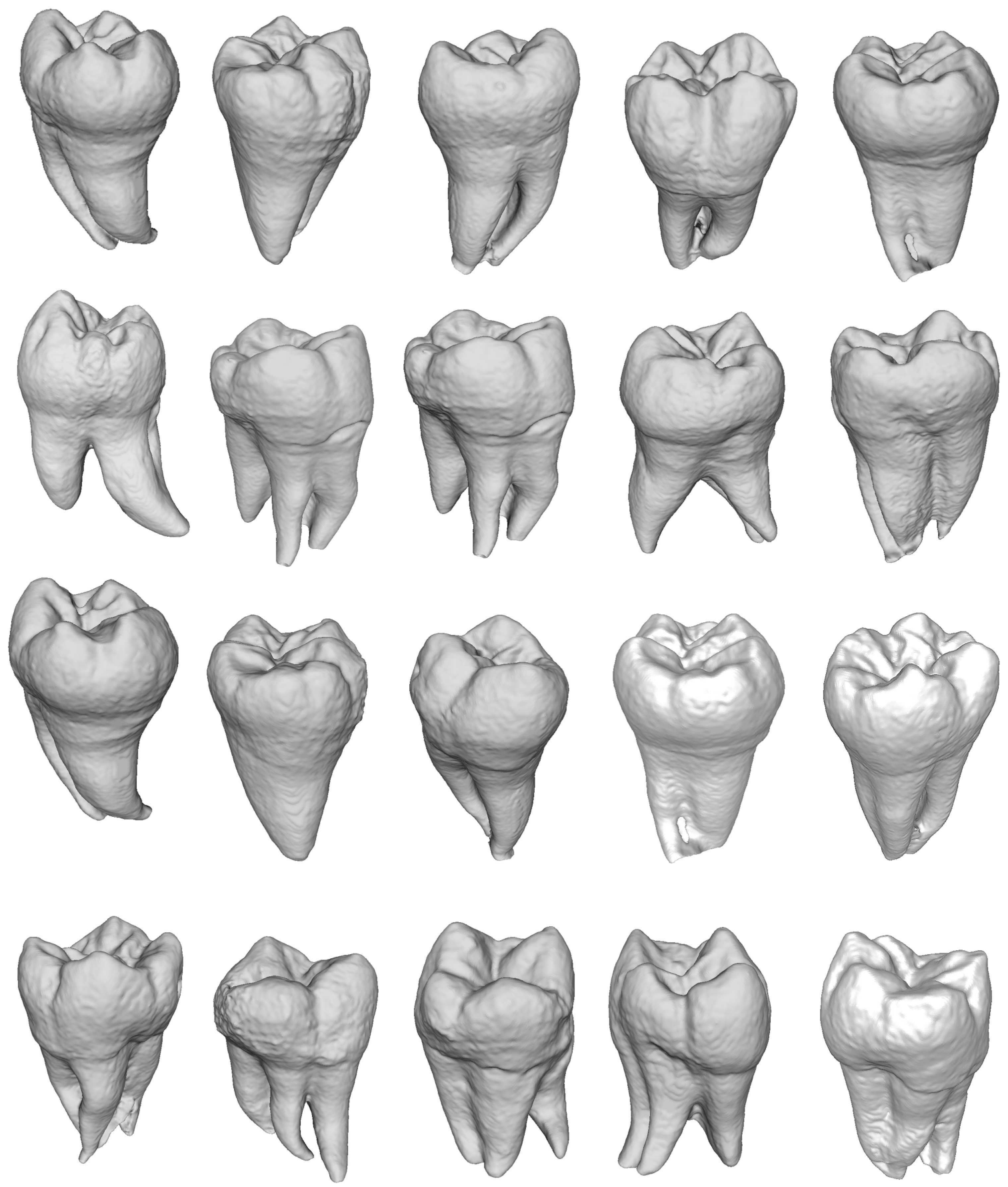

FIGURE 15: Sample individual teeth from the proposed 3D teeth library: (first row) maxillary deciduous teeth. (second row) maxillary molars. (third row) mandibular molars. (fourth row) mandibular third molars. 

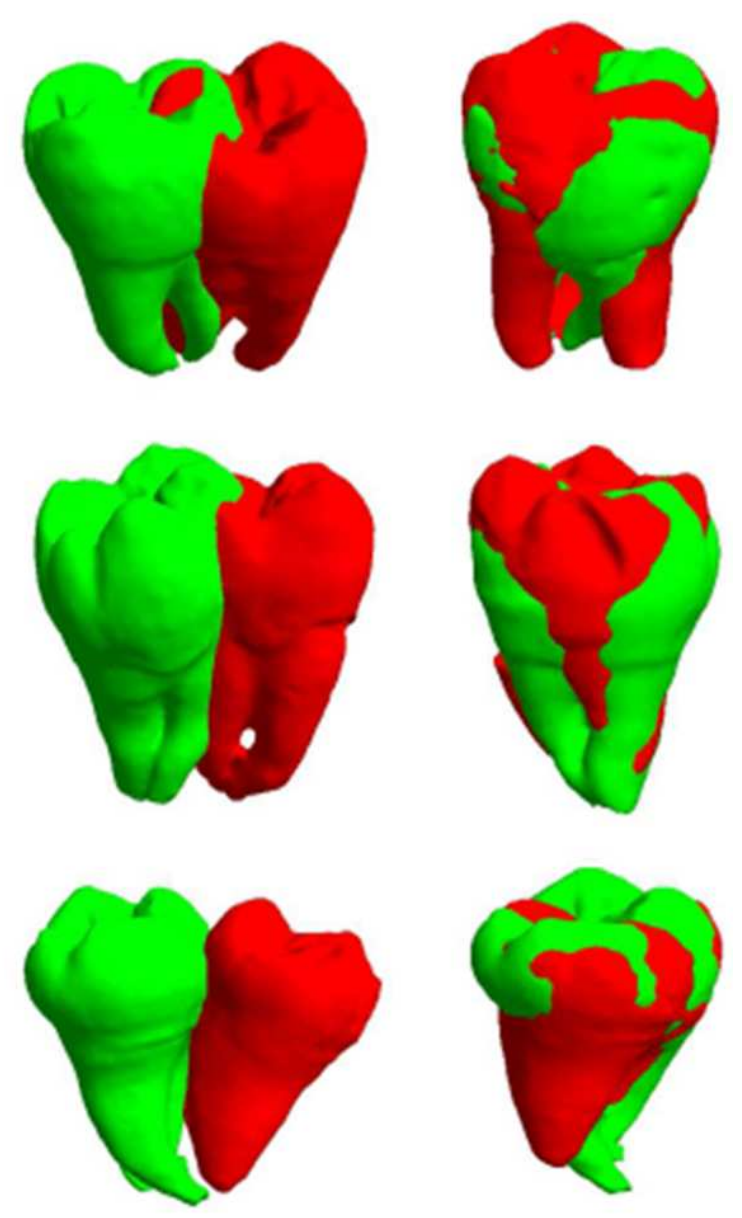

FIGURE 16: Three source models are visualized in red while the corresponding targets are shown in blue. First column show initial positions. Final registration results are demonstrated in the second column.

point cloud (see Figure 16).

Algorithm 1 summarizing the steps of ICP approach.

\section{Jaw Database Construction}

Table 1 shows the database constructed from 52 upper jaw molds belonging to 33 males and 19 females with on average age of 20 years old. Whereas lower jaw models are constructed from 58 lower jaw molds belonging to 33 males and 25 females with on 


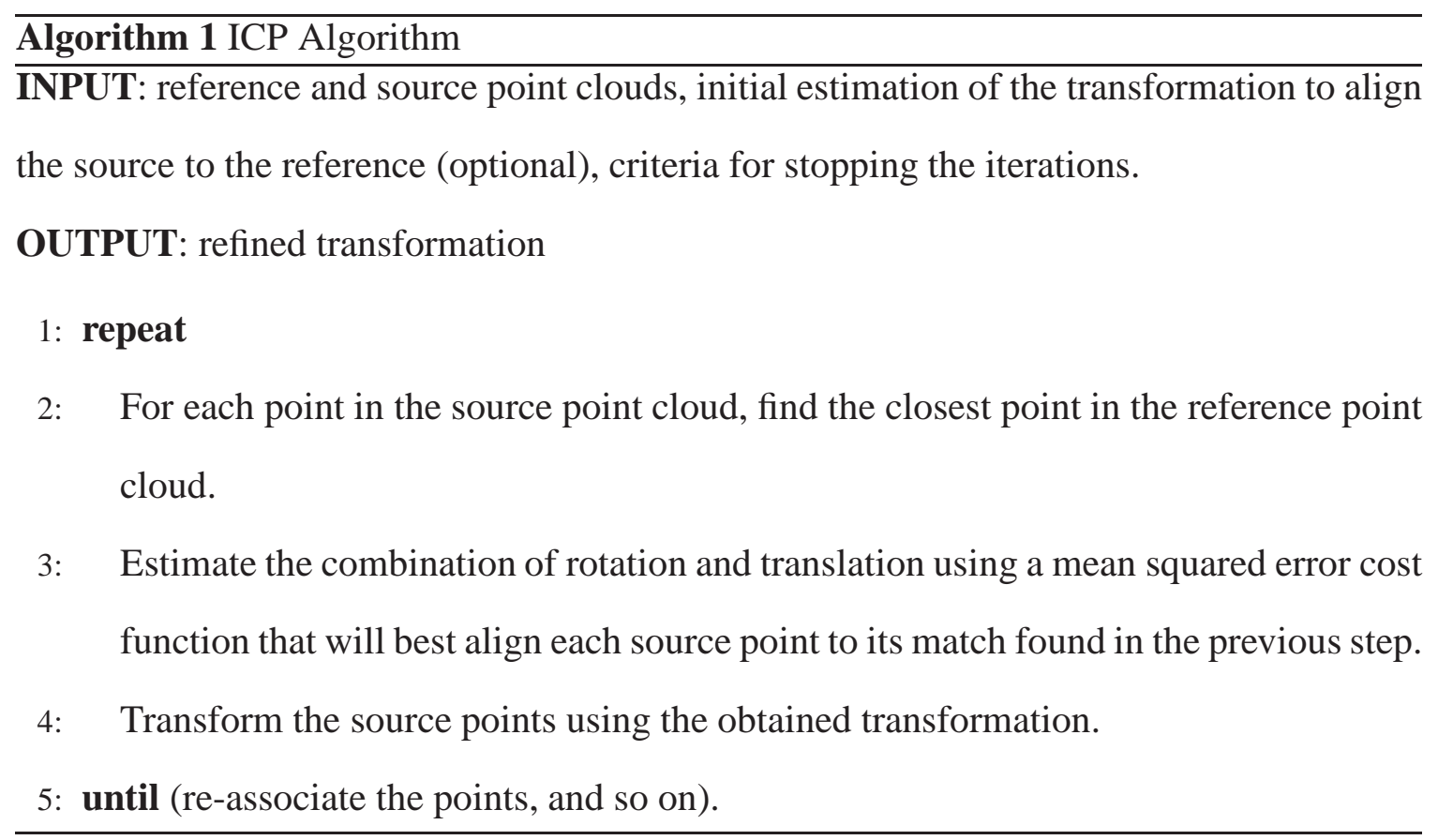

TABLE 1: Database construction of the human jaw: subjects categorized with respect to gender, age and ethnicity

\begin{tabular}{|c|c|c|c|c|c|c|c|}
\hline & \multirow{2}{*}{$\begin{array}{c}\text { number of } \\
\text { teeth }\end{array}$} & \multirow{2}{*}{ Age } & \multicolumn{2}{|c|}{ Gender } & \multicolumn{2}{|c|}{ Race } & \multirow{2}{*}{$\begin{array}{c}\text { Total } \\
\text { number } \\
\text { of Jaws }\end{array}$} \\
\hline & & & Males & Females & Blacks & Whites & \\
\hline \multirow{2}{*}{$\begin{array}{l}\text { Upper } \\
\text { Initial }\end{array}$} & 12 & $16-46(\mu=22.4, \sigma=9.4)$ & 10 & 15 & 10 & 15 & \multirow{4}{*}{104} \\
\hline & 14 & $15-46(\mu=20.3, \sigma=7.4)$ & 14 & 20 & 6 & 18 & \\
\hline \multirow{2}{*}{$\begin{array}{c}\text { Upper } \\
\text { Final }\end{array}$} & 12 & $16-21(\mu=17.2, \sigma=1.6)$ & 15 & 13 & 13 & 15 & \\
\hline & 14 & $14-46(\mu=20.2, \sigma=9.4)$ & 15 & 12 & 7 & 20 & \\
\hline \multirow{2}{*}{$\begin{array}{l}\text { Lower } \\
\text { Initial }\end{array}$} & 12 & $11-19(\mu=16.6, \sigma=2.1)$ & 19 & 15 & 14 & 20 & \multirow{4}{*}{126} \\
\hline & 14 & $15-46(\mu=23.8, \sigma=9.5)$ & 20 & 10 & 13 & 17 & \\
\hline \multirow{2}{*}{$\begin{array}{c}\text { Lower } \\
\text { Final }\end{array}$} & 12 & $14-19(\mu=16.6, \sigma=1.5)$ & 15 & 12 & 10 & 17 & \\
\hline & 14 & $13-46(\mu=19.5, \sigma=8.46)$ & 15 & 20 & 10 & 25 & \\
\hline
\end{tabular}

average age of 19 years old ${ }^{2}$.

The triangular meshes of the training ensemble are obtained from a high resolution computer tomography $\left(\mathrm{CT}^{3}\right)$ scan of human jaw molds where the Expectation-Maximization

\footnotetext{
${ }^{2} \mathrm{~A}$ key requirement for successful statistical SFS is the availability of a comprehensive database that describe the teeth/jaw variability per age, gender and ethnic factors. Ongoing efforts aim to undertake such a task make the database available for researchers worldwide.

${ }^{3}$ A Cone-beam CT (KODAK 9000 3D Extraoral) scanner is used.
} 


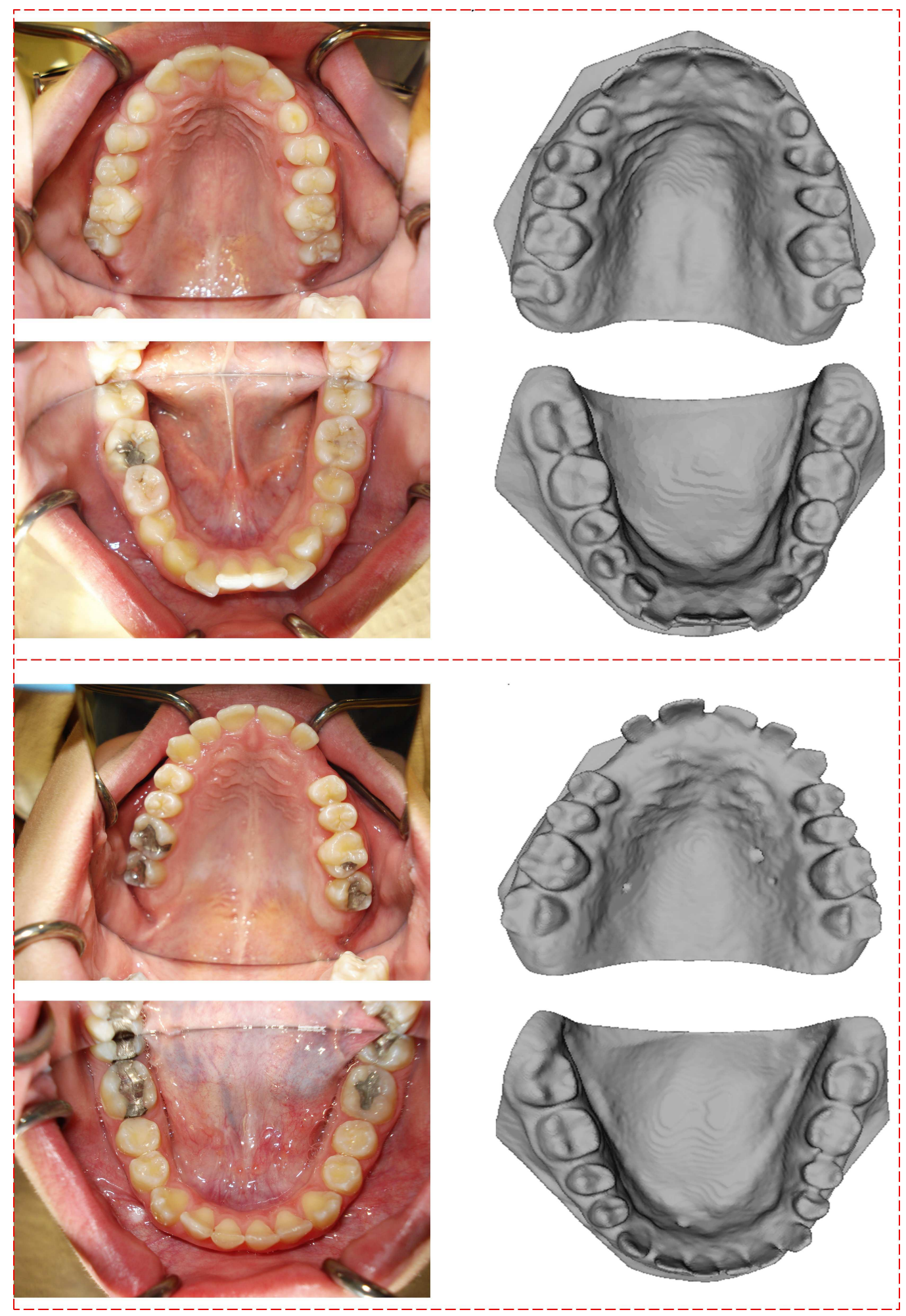

FIGURE 17: Sample of the human jaw (pre-repair) lower and upper jaws: first column shows the 2D images, 3D scans using cone beam CT machine shows in the second column. 


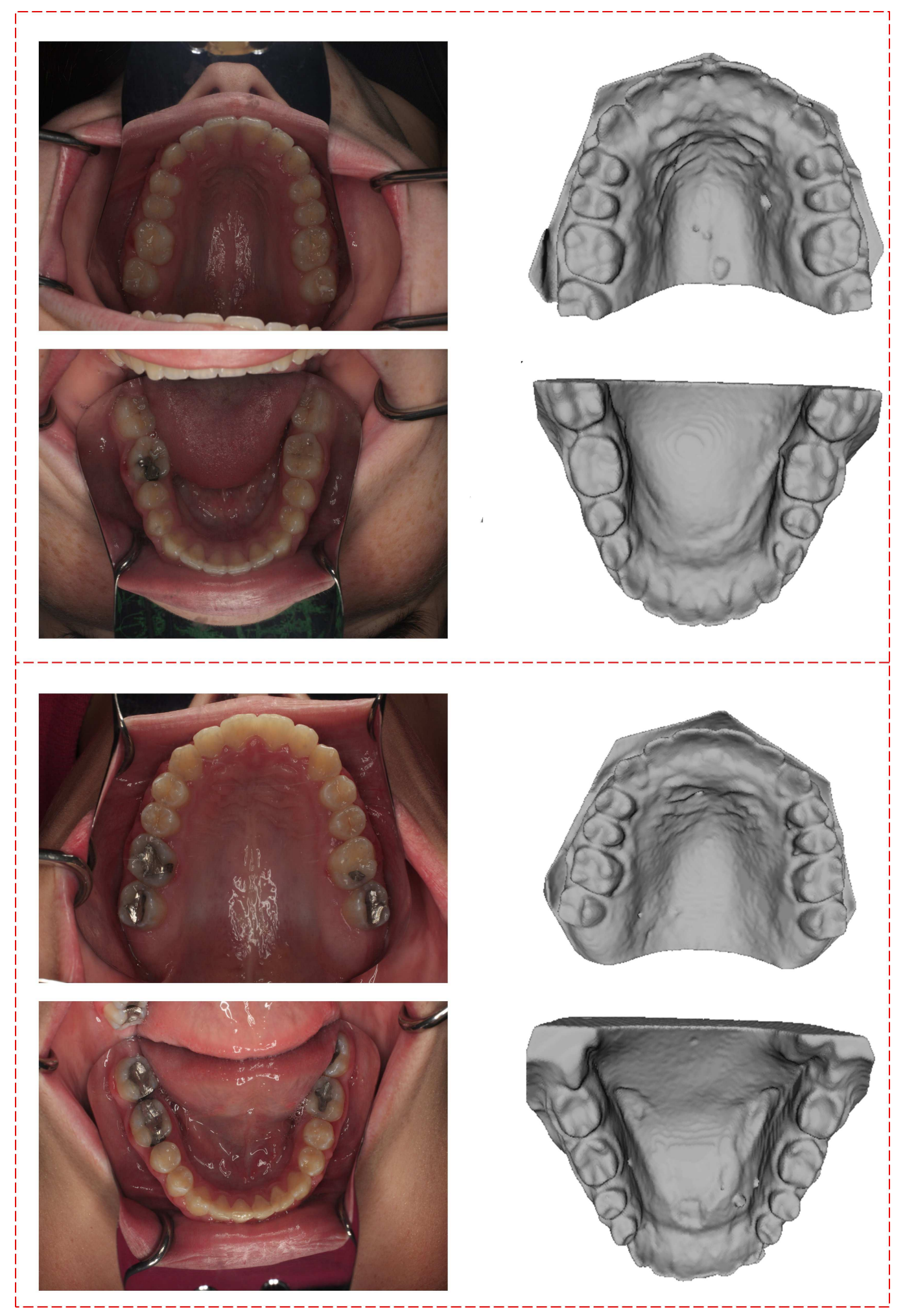

FIGURE 18: Sample of the human jaw (post-repair ) lower and upper jaws: first column shows the 2D images, 3D scans using cone beam CT machine shows in the second column. 

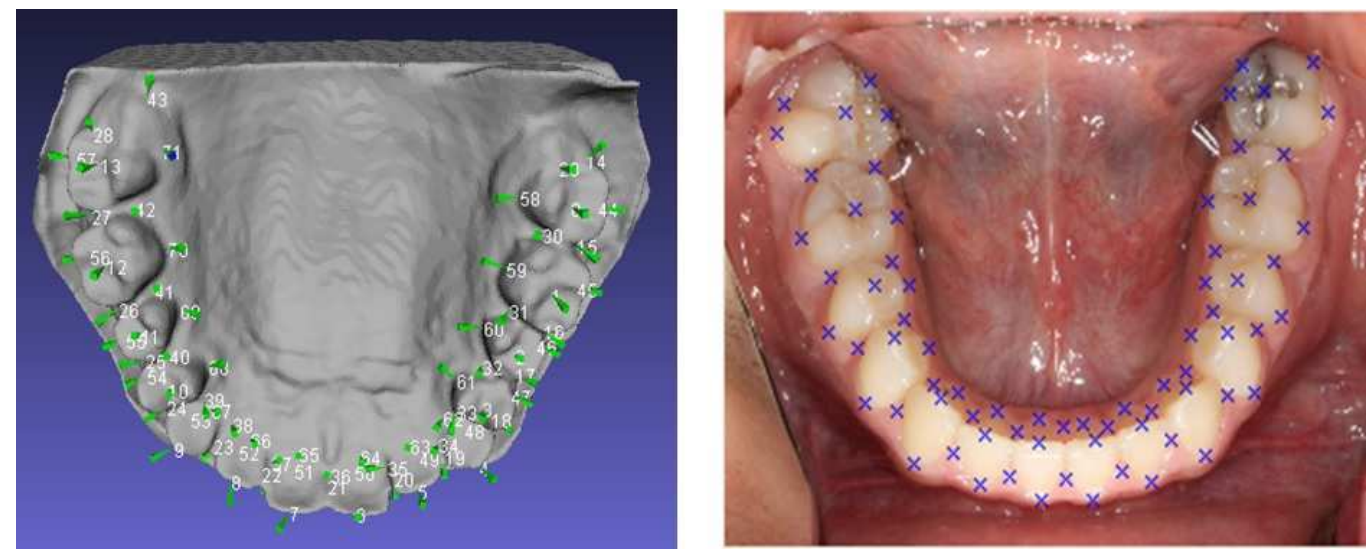

FIGURE 19: Illustration of the jaw anatomical landmarks.

(EM) algorithm is used for segmentation. Dense correspondence between jaw samples is obtained using the 3D thin-plate spline where the alignment procedure is guided by the sparse set of anatomical jaw landmarks as shown in Figure 19. The 3D thin-plate spline is used to provide a warping function between image pixels (assumed to be on the xy-plane in the 3D space) and surface points using image landmarks and surface landmarks as control points. Orthographic projection is applied to re-represent the triangular meshes in terms of Monge patches which provides a bijective mapping between surface points and image coordinates.

\section{E. Summary}

In this chapter, a system for building a database of real human teeth 3D models is presented. Teeth are scanned using Cone-beam CT with a resolution of $0.2 \times 0.2 \times 0.2 \mathrm{~mm}^{3}$. 


\section{CHAPTER IV}

\section{OCCLUSAL SURFACE RECONSTRUCTION OF HUMAN TEETH FROM A SINGLE IMAGE}

Image formation involves understanding sensor characteristics and object reflectance. In dentistry, an accurate 3D representation of the human jaw may be used for diagnostic and treatment purposes. Photogrammetry can offer a flexible, cost effective solution for accurate $3 \mathrm{D}$ representation of the human teeth, which can be used for diagnostic and treatment purposes. Nonetheless there are several challenges, such as the non-friendly image acquisition environment inside the human mouth and problems with lighting and errors due to the data acquisition sensors. In this chapter, the focus on the 3D surface reconstruction aspect for human teeth based on a single image. A more realistic formulation of the SFS problem is introduced by considering the image formation components: the camera, the light source, and the surface reflectance. A non-Lambertian SFS algorithm under perspective projection is proposed which benefits from camera calibration parameters. The attenuation of illumination due to near-field imaging taken into account . The surface reflectance is modeled using the Oren-Nayar-Wolff model which accounts for the retro-reflection case. Experiments provide promising quantitative metric results for the proposed approach.

\section{A. Introduction}

Modern dentistry requires the accurate 3D representation of the teeth and jaw for diagnostic and treatment purposes. For instance, orthodontic treatment involves the application, over time, of force systems to teeth for malocclusion correction. Oral and max- 
illofacial radiology can provide the dentist with abundant 3D information about the jaw. Several existing 3D systems for dental applications found in literature rely on obtaining an intermediate solid model of the jaw (cast or teeth imprints) and then capturing the 3D information from that model. Due to the lack of surface texture, SFS algorithms have been used to obtain such 3D tooth reconstructions due to the significant shading cue presented in a tooth image, e.g. $[6,20]$.

Photogrammetry seems to offer a flexible, cost effective solution while avoiding the need for castings. Nonetheless, intra-oral photogrammetric measurement is inherently difficult due to non-friendly image acquisition environments with lighting problems, specularity effects due to saliva, inevitable subject motion and errors due to the data acquisition sensors $[18,89]$. Hence the common assumptions of the image formation process for typical shape reconstruction algorithms are hardly valid, e.g. Lambertian reflectance and distant light source.

Starting from the pioneering work of Horn [90], shape recovery from a single image usually involves two steps; deriving an image irradiance equation under a certain set of assumptions related to the image formation process and designing a numerical scheme to solve such an equation for the underlying shape. Most of the SFS approaches (e.g. see [17]), however, focus on the computational part of the SFS problem, i.e. the numerical solution. As a result, the imaging model in most conventional SFS algorithms has been simplified under three simple, but restrictive assumptions: (1) the camera performs an orthographic projection of the scene, (2) the surface has a Lambertian reflectance and (3) the light source is a single point source at infinity. Unfortunately, such assumptions are no longer held in the case of intra-oral imaging environment for human teeth.

This chapter introduces a more realistic formulation of SFS by considering all the components of the problem, namely: the camera, the light source, and the surface reflectance. For the camera and the light source used the same modeling as in [91]. The camera is modeled by perspective projection (see Figure 20) with the camera parameters 
being known from an off-line calibration process and incorporated in the SFS formulation. The light source is assumed to be located at the optical center of the camera, i.e. retroreflection situation (see Figure 21) where the object is illuminated in the viewing direction. Under this near-field imaging, the attenuation of illumination due to the distance between the light source and the surface is taken into account, hence the method can deal with concave/convex ambiguity. Accounting for departures from Lambertian reflectance due to surface roughness, A modified Oren-Nayar-Wolff model is used [92] where surface roughness is physically measured using optical surface profiler (see Figure 22). The Oren-Nayar model [93] modulates the Lamberts cosine law by a term that depends on the squared sine of the incidence angle, resulting in apparent brightening at surface patches which move away from the light source; assumed to be in self shadow. Wolff, on the other hand, has a physically deeper model for diffuse reflectance from shiny but slightly rough surfaces. The model uses an angle dependent Fresnel term to account for the refractive attenuation of incident light at the surface-air boundary [94]. This Fresnel term modifies the Lambertian cosine model in a multiplicative way. The effect is to depress the surface radiance for near-normal incidence. 


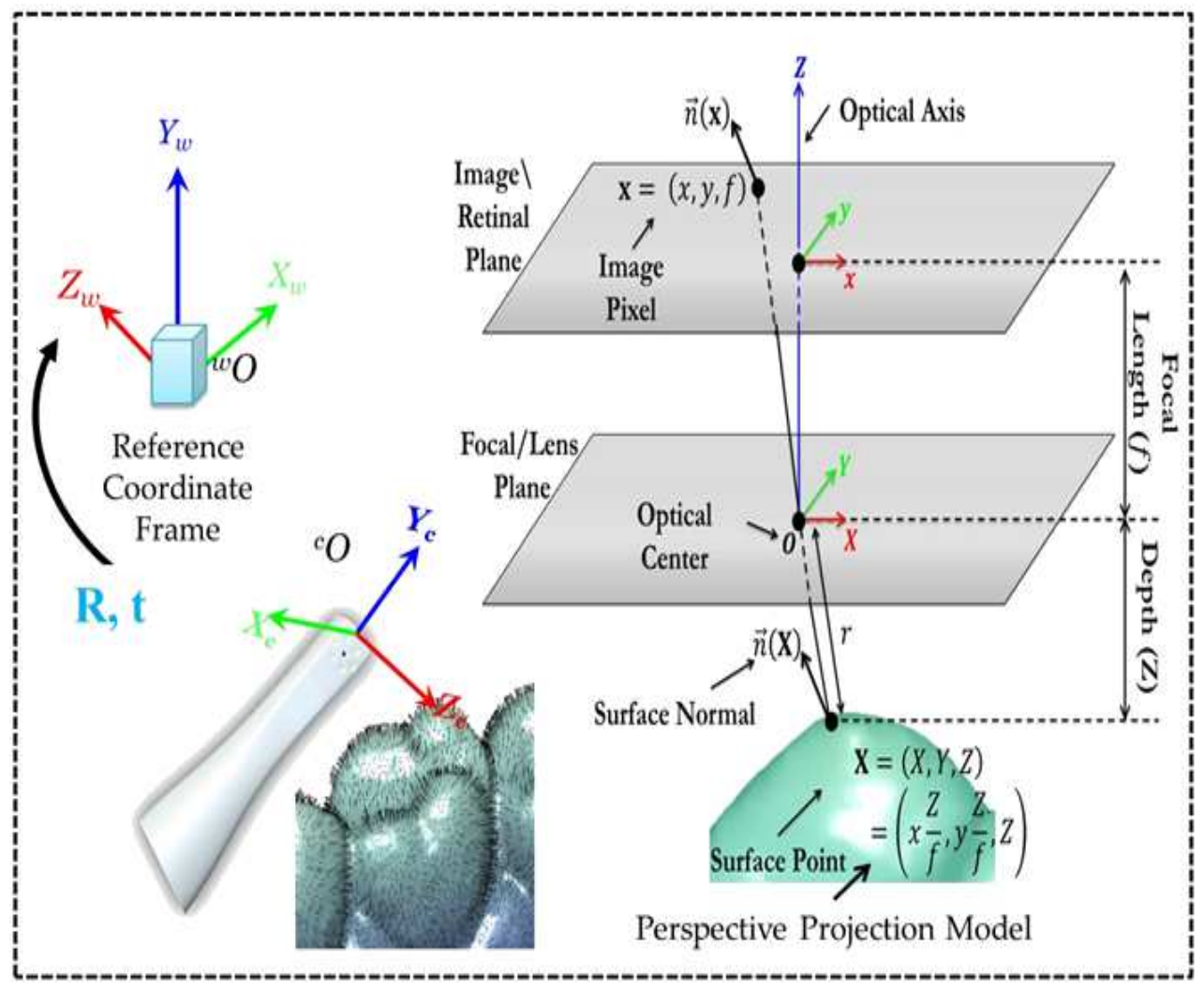

FIGURE 20: A calibrated wireless intraoral camera equipped by a motion tracker and Perspective projection camera model. 


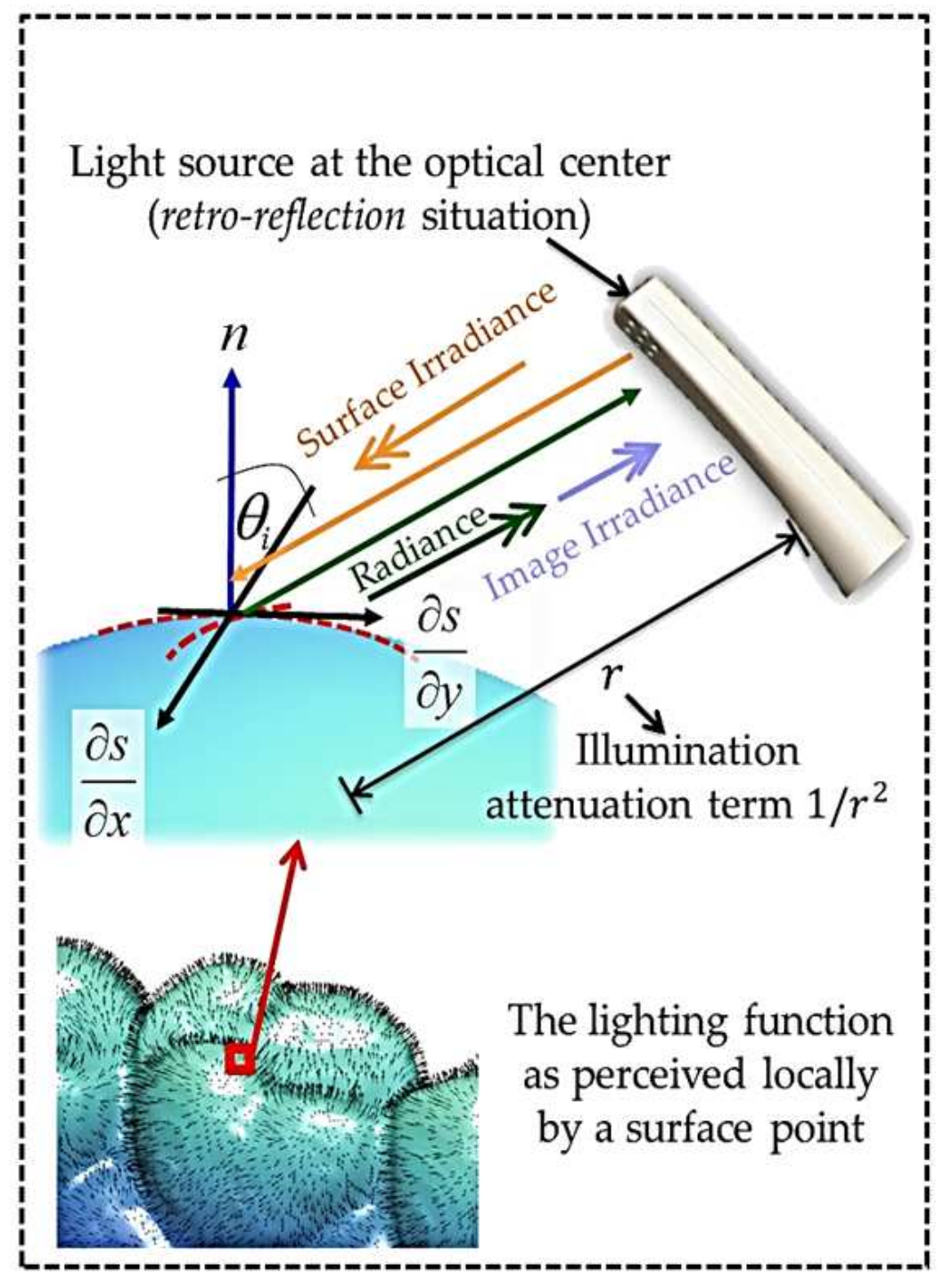

FIGURE 21: Local illumination model where object is illuminated in the viewing direction, i.e. retro-reflection case. 


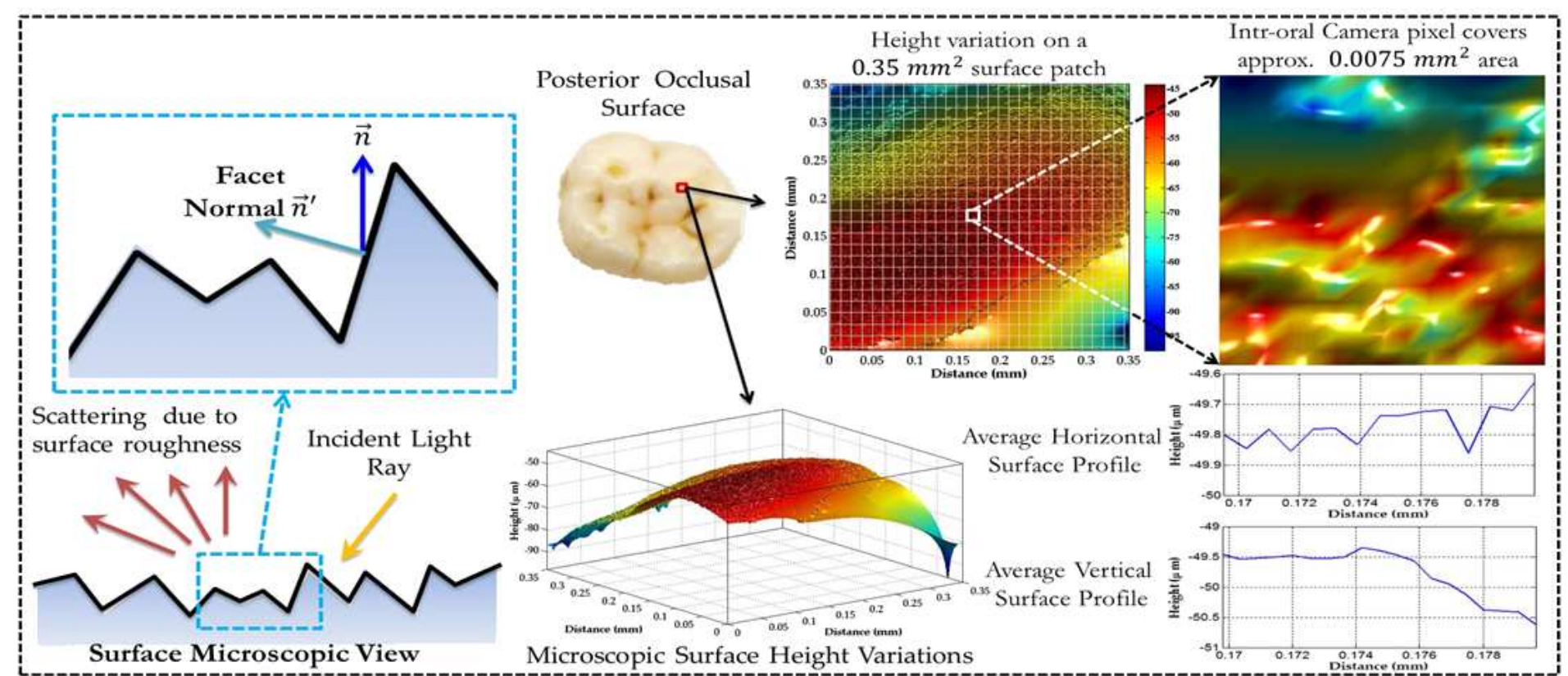

FIGURE 22: Surface reflectance properties where rough surfaces tend to scatter incident light as compared to smooth surfaces. Microscopic surface height variations of a posterior occlusal surface is measured by an optical surface profiler. 
The numerical solution, classified as Minimization-based [17], is based on the Taylor series expansion of the image irradiance equation followed by the application of the Jacobi iterative method. On the other hand, the image irradiance equation can be formulated as a partial differential equation (PDE) to solve for surface gradients, where the theory of viscosity solutions for Hamilton-Jacobi type equations provide a good framework of SFS algorithms [17]. However explicit PDE formulation of the SFS problem imposes regularity of the image irradiance function which is assumed to be continuous [17]. Nonetheless, human teeth do not fit such an assumption, due to the geometrical structure of the occlusal surface in particular, which forms attached and cast shadows in the captured image causing image discontinuities.

Carter et al. [6] evaluated three SFS models for artificial tooth surface reconstruction based on the work of Ahmed and Farag [26]. They concluded that, based on the quantitative error analysis, a perspective camera projection with an Oren-Nayar reflectance model has been proved to be the most ideal SFS formulation for extracting tooth crown surface from a single image. Nonetheless, their work did not incorporate the available camera parameters from their acquisition setup nor the object physical characteristics into the SFS-PDE formulation. The proposed SFS approach is compared with a non-Lambertian PDE-based approach $[6,12]$ via quantitative error metric derived from groundtruth teeth surfaces obtained from a CT-scanner. Vis-à-vis dental applications, the results demonstrate a significant increase in accuracy in favor of the proposed approach. In particular, the proposed approach is able to recover geometric details of the tooth's occlusal surface as compared to PDE-based approaches.

\section{B. Image Irradiance Equation}

According to the microscopic view of occlusal surface height variations, Figure 22, tooth surface reflectance can be modeled by micro-facet reflectance models where the 
Oren-Nayar-Wolff model is well-suited for the retro-reflection case [94]. When the object is illuminated in the viewing direction, taking into account the illumination attenuation term $\left(1 / r^{2}\right)$ as shown in Figure 21, the expression of the image irradiance $E$ using OrenNayar-Wolff model can be simplified to [92]:

$$
\begin{array}{r}
E(\mathbf{x})=\frac{A(1-F(\theta, \eta))^{2} \cos \theta+B \sin ^{2} \theta}{r^{2}} \\
\text { s.t. } A=1-0.5 \frac{\sigma^{2}}{\sigma^{2}+0.33}, B=0.45 \frac{\sigma^{2}}{\sigma^{2}+0.09}
\end{array}
$$

where $\mathbf{x}=(x, y)^{T}$ is the 2D point, $r$ is the distance to the light source [92], the parameter $\sigma$ denotes the standard deviation of the Gaussian distribution which is used as a measure of the surface roughness, $\theta$ is the inclination angle of the viewer/source and $F$ refer to the Fresnel reflection function [92] with refractive index of $\eta$.

\section{Parametric SFS for Non-Lambertian Surfaces}

A surface point in the 3D space can be related to its corresponding position in the image plane through the camera intrinsic (solved for once) and extrinsic (updated while the camera is in motion) parameters. The relation between a $3 \mathrm{D}$ point $\mathbf{X}=(X, Y, Z)^{T}$ and the corresponding point in the image coordinates $\mathbf{x}=(x, y)^{T}$ is written as $s(\mathbf{x}) \overrightarrow{\mathbf{x}}=P \mathbf{X}+b$, where $s$ is a scalar parameterized by the image pixel coordinates, $P$ is a $3 \times 3$ camera matrix, $b$ is a $3 \times 1$ translation vector and $\overrightarrow{\mathbf{x}}=\left[\mathbf{x}^{T} 1\right]^{T}$ is the extended vector defined in homogeneous coordinates. Therefore the point in $3 \mathrm{D}$ coordinates can be written as a function of the corresponding point in the image plane as,

$$
\mathbf{X}=P^{-1}[s(\mathbf{x}) \overrightarrow{\mathbf{x}}-b]=g(s(\mathbf{x}))
$$

Equation (14) represents the line in 3D passing through the optical center and the 
projected point $\mathbf{x}$ in the image plane. Thus solving for the scalar $s(\mathbf{x}) \forall \mathbf{x} \in \mathcal{D}$, using the available shading cues in the image domain $\mathcal{D} \in \mathbb{R}^{2}$, accounts for defining a unique 3D point $\mathbf{X}$ on the object's surface. The inherent relationship between the scale factor $s(\mathbf{x})$ and the surface normal vector $\vec{n}(\mathbf{x})$ can be expressed in terms of surface gradients $(p(\mathbf{x}), q(\mathbf{x}))$ in the gradient space [95], where $p(\mathbf{x})=\frac{\partial g(s(\mathbf{x}))}{\partial x}=p(s)$ and $q(\mathbf{x})=\frac{\partial g(s(\mathbf{x}))}{\partial y}=q(s)^{4}$. The image irradiance $E($.$) now becomes a function of the scalar s(\mathbf{x})$ defined in (14). In the sequel, the proposed variational formulation for the problem is presented in case of near illumination with Oren-Nayar-Wolff reflectance. A small pattern used in the calibration of the intraoral camera; the size of the pattern is suitable to size of the tooth.

\section{SFS Minimization Functional}

The SFS problem can then be formulated as finding the scalar $s$ which satisfies both the brightness constraint and the smoothness constraint ${ }^{5}$. While the former indicates the total brightness error of the reconstructed image, given the imaging process parameters, compared to the input image $I$, the latter is included to obtain a smooth surface that is free from discontinuities through penalizing the derivative of the surface.

This can be solved using a Taylor's series expansion of the estimated brightness $E(s)$ around $E\left(s^{n-1}\right)$, where $n$ is the iteration index. This is followed by applying the Jacobi iterative method. After $n$ iterations, for each point $\mathbf{x}$ in the image, $s^{n}(\mathbf{x})$ is given as,

$$
s^{n}(\mathbf{x})=s^{n-1}(\mathbf{x})+\lambda_{1} \frac{-I(\mathbf{x})+E\left(s^{n-1}(\mathbf{x})\right)}{\frac{d}{d s(\mathbf{x})} E\left(s^{n-1}(\mathbf{x})\right)}+\lambda_{2} \nabla s^{n-1}(\mathbf{x})
$$

where $\lambda_{1}$ and $\lambda_{2}$ are real positive coefficients defining the brightness and smoothness

\footnotetext{
${ }^{4}$ For notational simplicity $s$ is used to denote $s(\mathbf{x})$

${ }^{5}$ Since we are not solving for surface gradients explicitly, the integrability constraint is not considered in this work.
} 
factors respectively. The derivative of the image brightness w.r.t. $s$ can be written as,

$$
\begin{aligned}
& \frac{d}{d s(\mathbf{x})} E\left(s^{n-1}(\mathbf{x})\right)= \\
& \quad \frac{1}{r^{2}}\left\{\left[A\left(1-\cos ^{-1}(\vec{n}(\mathbf{x}) \cdot \vec{l})\right)^{2}\left(\frac{d \vec{n}(\mathbf{x})}{d s(\mathbf{x})} \cdot \vec{l}\right)\right.\right. \\
& \left.+\quad 2(\vec{n}(\mathbf{x}) \cdot \vec{l})\left(1+\cos ^{-1}(\vec{n}(\mathbf{x}) \cdot \vec{l}) \frac{\frac{d \vec{n}(\mathbf{x})}{d s(\mathbf{x})} \cdot \vec{l}}{\sqrt{\left(1-\left(\vec{n}(\mathbf{x}) \cdot \vec{l}^{2}\right)\right.}}\right)\right] \\
& \left.-\quad 2 B(\vec{n}(\mathbf{x}) \cdot \vec{l})\left(\frac{d \vec{n}(\mathbf{x})}{d s(\mathbf{x})} \cdot \vec{l}\right)\right\}
\end{aligned}
$$

where,

$$
\begin{aligned}
\frac{d \vec{n}(\mathbf{x})}{d s(\mathbf{x})} & =\frac{d \vec{v}(s)}{d s(\mathbf{x})} \frac{1}{\sqrt{\vec{v}^{T}(s) \vec{v}(s)}} \\
& -\frac{\vec{v}(s)}{\sqrt{\left(\vec{v}^{T}(s) \vec{v}(s)\right)^{3}}}\left(\vec{v}^{T}(s) \frac{d \vec{v}(s)}{d s(\mathbf{x})}\right)
\end{aligned}
$$

with $\vec{v}(s)=p(s) \times q(s)$. Once $s(\mathbf{x})$ is evaluated, the corresponding point location in the 3D space can be determined using (14). These step are enumerated in Algorithm 2.

\section{E. Experimental Results}

1. Testing Images Panel

To evaluate the $3 \mathrm{D}$ reconstruction results obtained with the proposed algorithm, several experiments are done on 250 real human teeth. Premolar models are constructed from 30 teeth. Mandibular molar models are constructed from 30 teeth. Mandibular third molar models are constructed from 120 teeth. Maxillary molar models are constructed from 


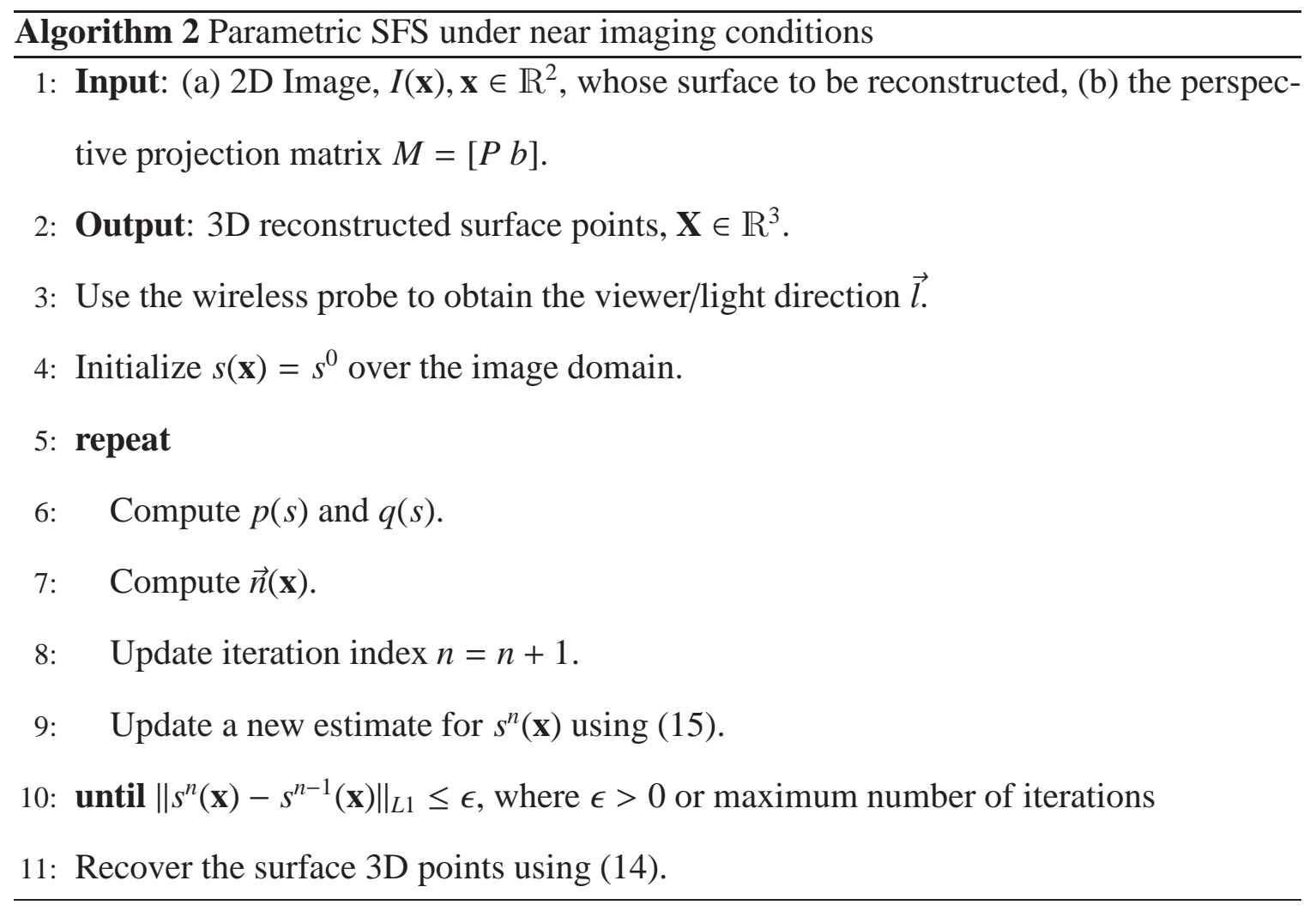

40 teeth. Whereas maxillary deciduous are constructed from 30 teeth. The wireless setup camera is used to acquire the images of an ensemble of real human teeth while the camera is held at a distance close to the crown surface to simulate the near-imaging condition inside the human mouth. A stylus arm with a touching probe (See Figure 23), with tolerance 0.001 $\mathrm{mm}$, is used to digitize the occlusal surface of this teeth ensemble to provide groundtruth metric information, even though sparse. Meanwhile CT scanning is performed to provide denser groundtruth information while maintaining the surface geometric details.

TABLE 2 summarizes the key differences between the proposed minimizationbased solution, terms as Algorithm A, and state-of-art PDE-based approaches, termed as Algorithms B and C, respectively.

The isosurface of the tooth scan is a metric reference to rigidly align the reconstructed 3D point from SFS to share the same metric coordinate frame. For alignment, the first and second moments are normalized [96] without affecting the scale. This initializes an ICP-based rigid registration algorithm [85]. Point correspondence between CT and the 


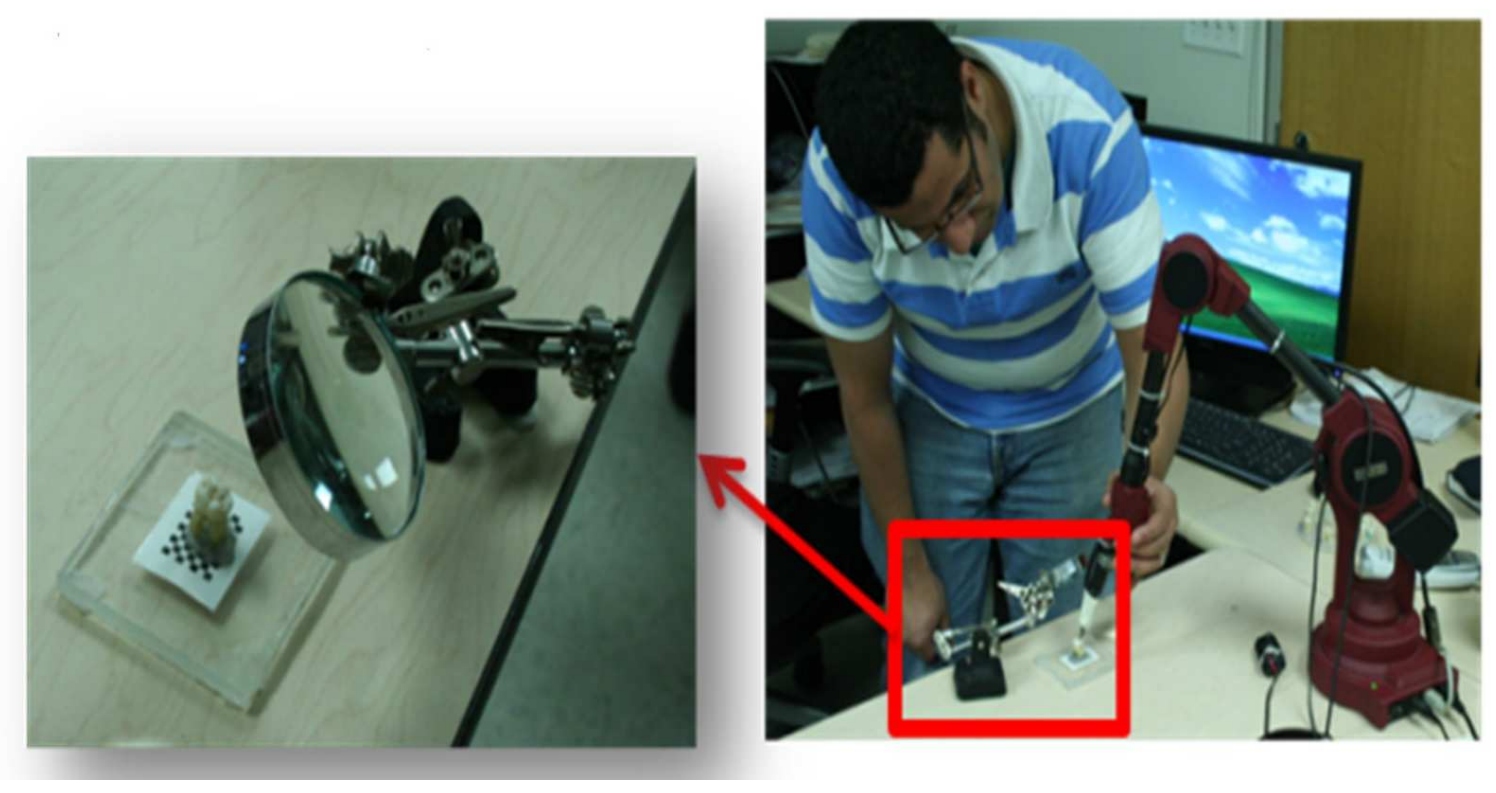

FIGURE 23: A stylus arm with a touching probe.

TABLE 2: SFS Algorithm Panel

\begin{tabular}{|c|c|c|c|}
\hline Algorithm & Reflectance Model & Camera Parameters & Numerical Solution \\
\hline \hline A-new & Oren-Nayar-Wolff & fully calibrated & Minimization-based \\
\hline B $[6,26]$ & Oren-Nayar & only unit focal length & PDE-based \\
\hline C [12] & Oren-Nayar-Wolff & only unit focal length & PDE-based \\
\hline
\end{tabular}




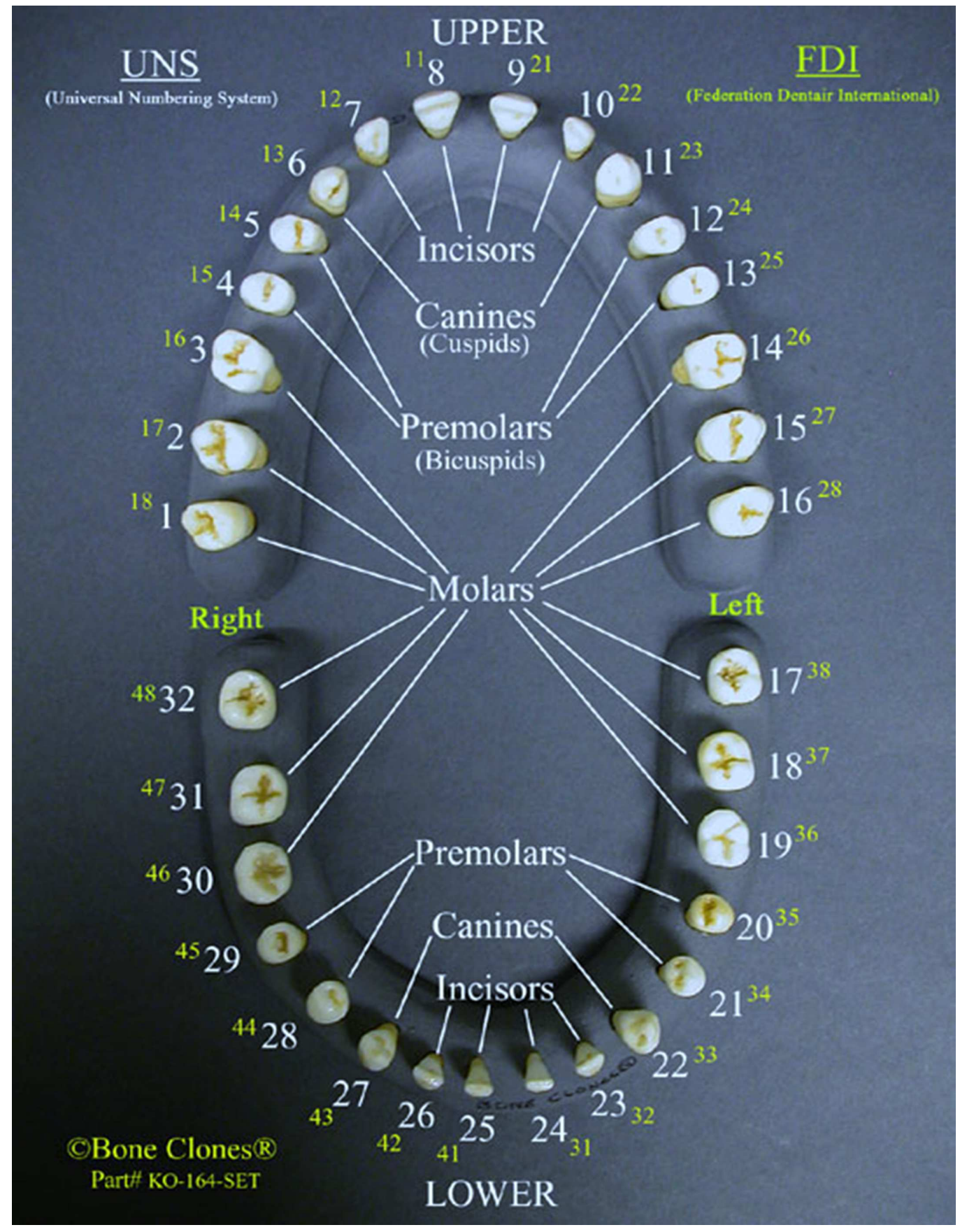

FIGURE 24: The 32 adult human teeth. 
TABLE 3: Overall surface reconstruction accuracy $(R M S)$ in $\mathrm{mm}$

\begin{tabular}{|c|c|c|c|}
\hline Tooth type & Algorithm A & Algorithm B & Algorithm C \\
\hline \hline Mandibular 3rd molars & 0.47232 & 1.07022 & 1.05386 \\
\hline Mandibular Molars & 0.63568 & 1.13321 & 1.12548 \\
\hline Maxillary Molars & 0.58694 & 0.89672 & 0.88411 \\
\hline Maxillary deciduous & 0.65997 & 1.11995 & 1.11456 \\
\hline Premolars & 0.45267 & 0.83282 & 0.81752 \\
\hline
\end{tabular}

reconstructed surface is obtained based on Hausdorff distance [97]. The SFS algorithms are compared in accordance with an error estimator based on the root mean square $(R M S)$ error between the 3D points from the $\mathrm{CT}$ scan and the corresponding reconstructed surface points. It is worth mentioning that throughout the experimentations, Algorithm A converges after 5-10 iterations while Algorithms B and C converge after 100 iterations on average. TABLE 3 shows the overall surface reconstruction accuracy of the three algorithms based on the testing images panel.

Figure 25 (fourth and fives rows) shows sample reconstructions of human teeth with different types using Algorithms B and $\mathrm{C}$, which were not able to recover the geometrical details of the occlusal surface when compared to the proposed minimization-based algorithm (Figure 25 (third row)). This emphasizes the role of incorporating physical surface characteristics (surface roughness in the proposed case ${ }^{6}$ ) along with sensor parameters in the process of shape recovery. More results shown in Figure 26 as applied on maxillary deciduous and premolar teeth.

\section{F. Summary}

This chapter focused on the surface reconstruction aspect of human teeth from a

\footnotetext{
${ }^{6}$ Throughout the experimentation, the average surface roughness parameter is used that computed from the surface profiler.
} 


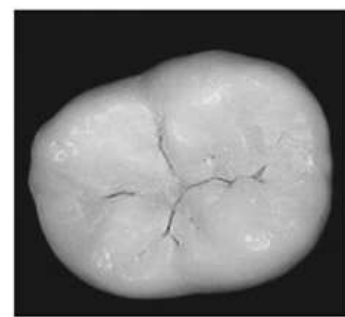

Mandibular $3^{\text {rd }}$ molar
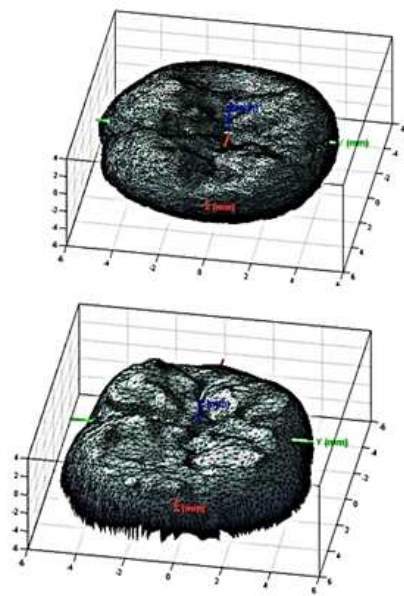

$R M S=0.43604 \mathrm{~mm}$

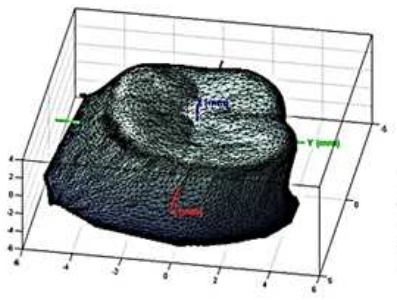

$R M S=1.09896 \mathrm{~mm}$

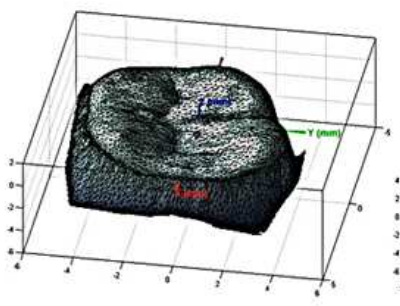

$R M S=1.07354 \mathrm{~mm}$

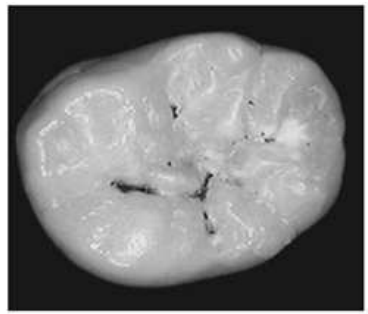

Mandibular molar
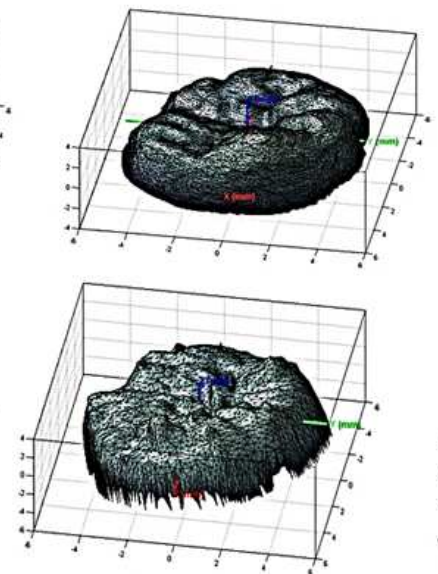

$R M S=0.47044 \mathrm{~mm}$

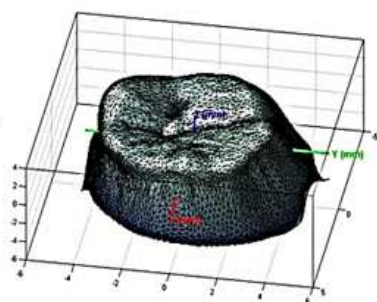

$R M S=0.99537 \mathrm{~mm}$

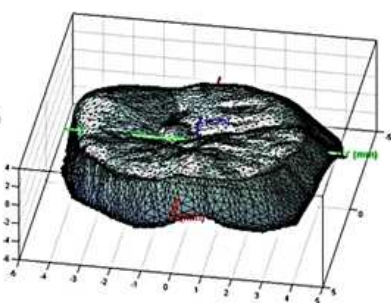

$R M S=0.95537 \mathrm{~mm}$

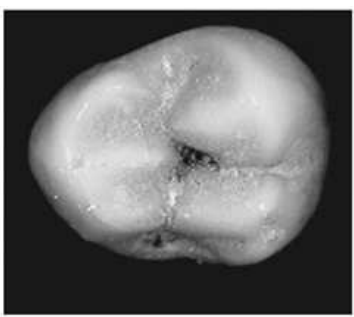

Maxillary molar
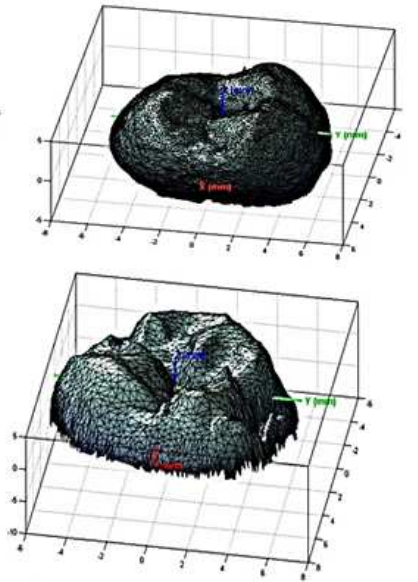

$R M S=0.66208 \mathrm{~mm}$

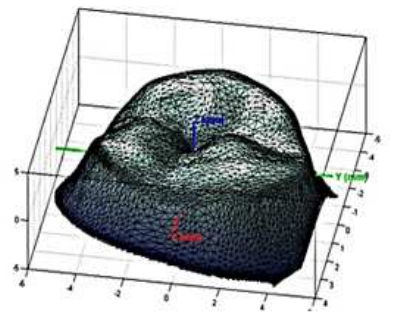

$R M S=1.01085 \mathrm{~mm}$

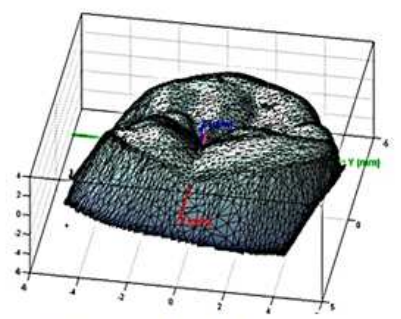

$R M S=0.97799 \mathrm{~mm}$

FIGURE 25: First row: images for three different types of human teeth (mandibular $3^{r d}$ molar, mandibular molar and maxillary molar), captured by the intra-oral camera. Second row: groundtruth occlusal surface generated from CT scanning. Third row: surface reconstruction based on algorithm A (proposed solution). Fourth row: surface reconstruction based on algorithm B. Last row: surface reconstruction based on algorithm C. Notice algorithms B and C (PDE-based) did not capture the geometric details of the occlusal surface as compared to the minimization-based algorithm A. 

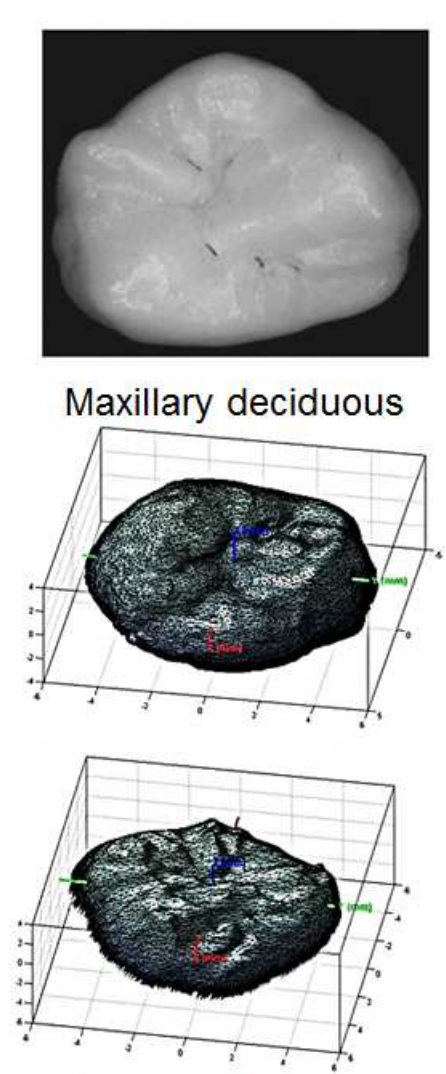

$R M S=0.45095 \mathrm{~mm}$

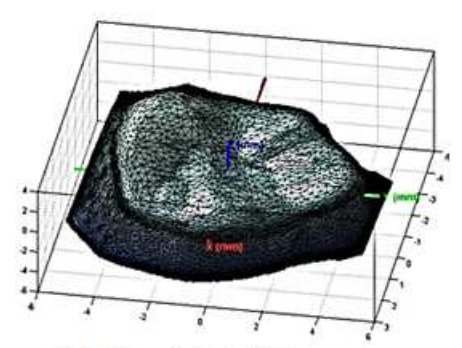

$R M S=1.02100 \mathrm{~mm}$

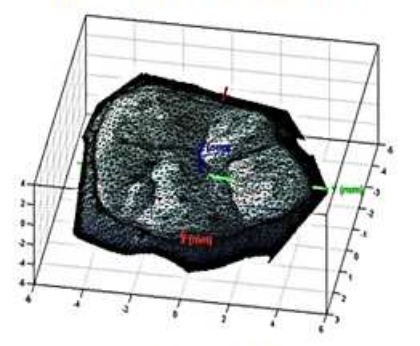

$R M S=1.01155 \mathrm{~mm}$
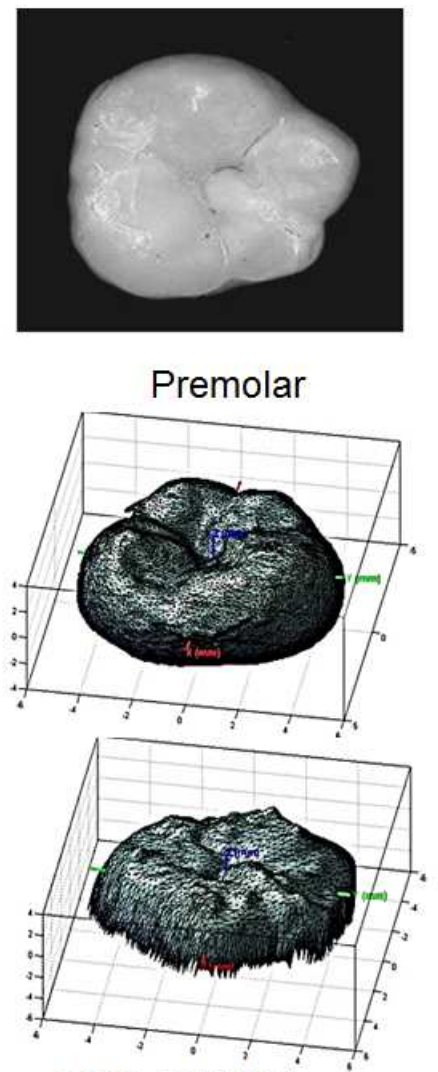

$R M S=0.36374 \mathrm{~mm}$

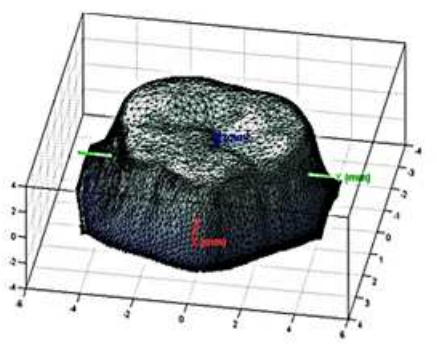

$R M S=0.99349 \mathrm{~mm}$

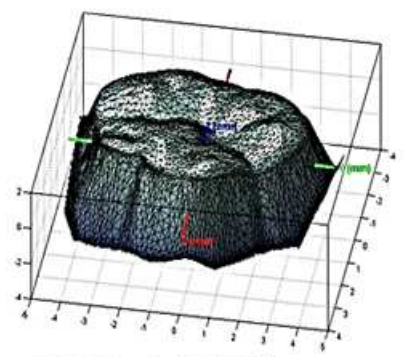

$R M S=0.97529 \mathrm{~mm}$

FIGURE 26: First row: images for more two different types of human teeth (maxillary deciduous and premolar), captured by the intraoral camera. Second row: groundtruth occlusal surface generated from CT scanning. Third row: surface reconstruction based on algorithm A (proposed solution). Fourth row: surface reconstruction based on algorithm B. Last row: surface reconstruction based on algorithm C. Notice algorithms B and C (PDEbased) did not capture the geometric details of the occlusal surface as compared to the minimization-based algorithm A. 
single image captured by an intraoral camera under near-field imaging. The work has addressed several challenges related to the image formation process including near illumination and perspective projection, while taking into account the deviation from the simplifying Lambertian assumption. Proposed formulation exploits all calibration information provided by the acquisition system setup. In particular, on average, the proposed approach reduces the error metric by $0.4434 \mathrm{~mm}$ compared to un-calibrated PDE-based SFS formulation. While the improvements are fractions of a millimeter, this is considered significant for dental-related applications such as tooth implants and surface analysis.

Ongoing efforts are directed towards reconstructing a complete metric model for the human jaw for various dentistry applications where a handy dental probe for data acquisition can provide ease-of-use for the dentist and is considered comfortable for the patient. This involves (1) handling inherited specular regions in tooth surface due to saliva and enamel reflectance and (2) performing surface registration and mesh zippering under partial overlaps (Chapter V). 


\section{CHAPTER V}

\section{D RECONSTRUCTION OF THE HUMAN JAW USING VARIATIONAL SFS AND FEATURE DESCRIPTORS}

This chapter proposes a new variational formulation that relates an evolving surface model with image information, taking into consideration that the image is taken by a perspective camera with known parameters. A new energy functional is formulated to incorporate brightness, smoothness, and integrability constraints. All of these terms assume a hyper surface that evolves in time to meet their criteria. Gradient descent optimization with Euler-Lagrange is used for optimization. Furthermore, a novel approach is proposed for 3D surface reconstruction of the human visible whole teeth [20] . Due to the difficulties of setting up a data acquisition system inside the mouth, an intraoral camera is used to capture a sequence of calibrated images. These images are registered together to build a panoramic view of the jaw. A SFS algorithm that benefits from camera calibration parameters is incorporated to build a 3D model from the panoramic image obtained from the previous stage. The proposed approach results in a 3D surface which has finer details compared with those resulting from other literature techniques. Also, different real and artificial visible whole teeth surface reconstructions are demonstrated to show the efficiency of the proposed system.

\section{A. Introduction}

Substantial efforts have focused recently on computerized diagnosis in dentistry [98]. Bernard et al. [99] developed an expert system where cephalometric measurements are ac- 
quired manually from the analysis of radiographs and plaster models. Laurendeau et al. [98] presented a computer-vision technique for the acquisition of jaw data from inexpensive dental wafers. That system was capable of obtaining imprints of the teeth. Usually, most of the 3D systems for dental applications found in the literature rely on obtaining an intermediate solid model of the jaw (cast or teeth imprints) and then capturing the 3D information from that model. User interaction is needed in such systems to determine the 3D coordinates of fiducial reference points on a dental cast. Other systems that can measure the 3D coordinates have been developed using either mechanical contact [100] or a travelling light principle [101, 102]. Goshtasby et al. [103] designed a range scanner based on white light to reconstruct the cast. The scanner used the subtractive light principle to create very thin shadow profiles on the cast.

An intraoral camera is used (see Figure 1) to capture a sequence of calibrated images which is more comfortable to patients. Also it does not require a long time to scan a jaw. The resulting sequence of images covers the jaw and contains overlapped image regions. These sequential images are taken in pairs to perform image alignment by estimating projective transformations. This process incorporates points correspondences accurately found by the affine and scale invariant transformation approach (known as ASIFT). After estimating the projective transformations, the image pairs are used together in order to build a panoramic image of the whole jaw. The new view is used to build a 3D surface using the SFS algorithm. The used SFS is depending on calibration parameters. A formulation of the SFS that uses intrinsic and extrinsic camera parameters is used such that a better surface [18] is obtained. This technique is compared with former approaches and the difference is significant. Incorporating camera parameters results in surfaces with fine details that can not be handled by the other methods that involve SFS. Different 3D reconstruction results of artificial jaws will be demonstrated to show the efficiency of the proposed technique. 


\section{B. Data Acquisition}

The human jaw is not a friendly environment for calibrated video measurements. There are problems with lighting (effect of saliva, tooth discolorization, gum texture, and other sources of specularity), motion (even inevitable slight motions of the upper/lower jaw may lead to errors far beyond the desired tolerance of submillimeters accuracy), and data acquisition time (it is not comfortable to open the jaw widely for over a minute or two). There are small cameras available that can provide viewing of the human jaw. The lenses are usually active and allocated on the dental probe to provide viewing not calibrated image capturing. Hence, a major problem to overcome would be the design of a small size CCD camera with passive or active lenses that can be calibrated with respect to a reference frame.

This chapter, an intraoral camera with an attached small light source is used to acquire 2D images of the teeth. Some specifications are given as: LED light source, 1/4" CCD Sensor; 320,000 pixels $\left(\mathrm{PAL}^{7}\right), 270,000$ pixels $\left(\mathrm{NTSC}^{8}\right)$ and resolution $512 \times 582$ (PAL); $512 \times 942$ (NTSC). The objective of this part is to remove global differences between two given images (source and target). Every two sequential images will be aligned together. The output of this process is a panoramic image resulting from putting (stitching) the aligned sequence of images for the whole jaw.

The description of registering two images by mapping points correspondences is given in this section. A point in the source image is related to its corresponding target position by a projective transformation [52]. Note that the overlap between the two images results from imaging the same object by the camera to a different positions. Given a set of $K$ homogeneous image point correspondences $\mathbf{C}_{o}^{s} \in \mathbb{R}^{3}$ (in the source image) and $\mathbf{C}_{o}^{t} \in \mathbb{R}^{3}$ (in the target) where $o \in 1,2, \ldots, K$ : a projective transformation $\mathbf{H}_{3 \times 3}$ is required to get estimated to map a source image point to its corresponding target image position by the relation $s_{o} \mathbf{C}_{o}^{t}=\mathbf{H C}_{o}^{s}$ where $s_{o}$ is a scaling coefficient for the projective transformation

\footnotetext{
${ }^{7}$ Phase Alternating Line.

${ }^{8}$ National Television System Committee.
} 
effect. Actually, this equation will lead to two linear equations: $h_{11} x_{o}^{s}+h_{12} y_{o}^{s}+h_{13}-h_{31} x_{o}^{t} x_{o}^{s}-$ $h_{32} x_{o}^{t} y_{i}^{s}-h_{33} x_{o}^{t}=0$ and $h_{21} x_{o}^{s}+h_{22} y_{o}^{s}+h_{23}-h_{31} y_{o}^{t} x_{o}^{s}-h_{32} y_{o}^{t} y_{o}^{s}-h_{33} y_{o}^{t}=0$ where $x$ and $y$ are the coordinates of point. A linear system of equations $\Psi \Theta=\mathbf{0}$ can be constructed where $\Theta=\left[h_{11} h_{12} \ldots h_{33}\right]$ is the parameters column of the projective transformation $\mathbf{H}$ and $\Psi$ is the coefficients matrix. The projective transformation parameters are estimated by solving this linear system through the singular value decomposition approach. If the points correspondences are accurate, the overall difference between the source and target images vanishes when they are fused together. The success of this process is highly dependent on selecting the source and target points correspondences.

\section{Affine Scale-Invariant Feature Transform(ASIFT)}

The ASIFT [104] is an affine invariant extension of SIFT. The ASIFT simulates three affine parameters, scale and changes of the camera axis orientation longitude angle and the latitude angle (which is equivalent to tilt) and normalizes the rest three parameters, rotation and translation. More specifically, the ASIFT simulates the two camera axis parameters, and then applies SIFT which simulates the scale and normalizes the rotation and the translation. For two stereo images the ASIFT algorithm can be summarized as follows: Each image is transformed by simulating all possible linear distortions caused by the change of orientation of the camera axis. These rotations and tilts are performed for a finite and small number of latitudes and longitudes. All simulated images are compared by a similarity invariant matching algorithm (SIFT) in the following manner: (1) scale-space peak selection, (2) key-point localization, (3) orientation assignment, (4) key-point descriptor. The scale space $L\left(x, y, \sigma_{s}\right)$ can be constructed by the linear convolution of the image I with Gaussian kernel $G\left(x, y, \sigma_{s}\right)$. Scale-space extrema detection searches over all scales $\sigma_{s}$ and image locations to identify potential interest points which are invariant to scale and orientation; this can be efficiently implements using Difference-of-Gaussians $D\left(x, y, \sigma_{s}\right)$ which takes the difference between consecutive scales, $D\left(x, y, \sigma_{s}\right)=L\left(x, y, \sigma_{s}\right)-L\left(x, y, \sigma_{s-1}\right)$, where 
a point $(x, y)$ is selected to be a candidate interest point if it is larger or smaller than its $3 \times 3 \times 3$ neighborhood system defined on $\left\{D\left(x, y, \sigma_{s-1}\right), D\left(x, y, \sigma_{s}\right), D\left(x, y, \sigma_{s+1}\right)\right\}$ where $\sigma_{s}$ is marked to be the scale of the point $(x, y)$. Then removal of points with low contrast and points that are localized along edges is accomplished.

In order to obtain a point descriptor $d$ which is invariant to orientation, a consistent orientation should be assigned to each detected interest point based on the gradient of its local image patch. The orientation is selected to be the peak of the weighted orientation histogram. Then a $16 \times 16$ image window surrounding the interest point $(x, y)$ is divided into sixteen $4 \times 4$ sub-window, an 8 -bin weighted orientation histogram is computed for each sub-window, hence ending up with $16 \times 8=128$ descriptors for each interest point. Thus each detected interest point can now be defined at location, specific scale, certain orientation $\theta$ and a descriptor vector as $\{x, y, \sigma, \theta, d\}$. Interest point matching is performed to provide correspondences between the given images. Two points are said to be in correspondence if their descriptors match in L2-norm. The overall performance is twice slower than SIFT, but it gives more matching rate especially with face images.

The resulting matched points from the ASIFT algorithm may include incorrectly matched points so RANSAC [105] is used to fit a transformation model to those points. A random sample consists of four points which is the minimal subset of the interest points sufficient to determine a projective transformation is selected. The transformation is estimated by solving a linear system with the chosen random sample, and then the resulting transformation is evaluated using all correspondence points available. These points which deviate from the current transformation model by a specified threshold are considered outliers; hence the support of the model will be measured by the ratio of the inlier to the total number of points. For n point correspondences, this procedure is repeated K-times, where $K \leq \frac{n !}{2 !(n-2) !}$ then the best fit transformation model is the one with the maximum support, and the points marked as outliers are excluded from the interest points. 


\section{EulerLagrange Equation}

The Euler-Lagrange differential equation [106] is the fundamental equation of calculus of variations. Euler's equation in calculus of variations is a differential equation whose solutions are the functions for which a given functional is stationary. The EulerLagrange equation was developed by Swiss mathematician Leonhard Euler and Italian mathematician Joseph-Louis Lagrange in the 1750s.

Due to a differentiable functional is stationary at its local maxima and minima, the EulerLagrange equation is useful for solving optimization problems in which, given

some functional, one seeks the function minimizing (or maximizing) it. This is analogous to Fermat's theorem in calculus, stating that at any point where a differentiable function attains a local extremum, its derivative is zero.

It states that if $J$ is defined by an integral of the form:

$$
J=\int f(t, y, \dot{y}) d t
$$

where

$\dot{y} \equiv \frac{d y}{d t}$,

then $J$ has a stationary value if the Euler-Lagrange differential equation:

$$
\frac{\partial f}{\partial y}-\frac{d}{d t}\left(\frac{\partial f}{\partial \dot{y}}\right)=0
$$

is satisfied.

If time-derivative notation $\dot{y}$ is replaced instead by space-derivative notation $y_{x}$, the equation becomes 


$$
\frac{\partial f}{\partial y}-\frac{d}{d t}\left(\frac{\partial f}{\partial y_{x}}\right)=0
$$

In many physical problems, $f_{x}$ (the partial derivative of $f$ with respect to $x$ ) turns out to be 0 , in which case a manipulation of the Euler-Lagrange differential equation reduces to the greatly simplified and partially integrated form known as the Beltrami identity,

$$
f-y_{x} \frac{\partial f}{\partial y_{x}}=C
$$

For three independent variables [107], the equation generalizes to

$$
\frac{\partial f}{\partial u}-\frac{\partial}{\partial x} \frac{\partial f}{\partial u_{x}}-\frac{\partial}{\partial y} \frac{\partial f}{\partial u_{y}}-\frac{\partial}{\partial z} \frac{\partial f}{\partial u_{z}}=0
$$

\section{E. Shape from shading using calibrated images}

The presented acquisition setup allows the knowledge of camera parameters as such the proposed SFS formulation takes this into account. A surface point in the 3D space can be related to its corresponding position in the image plane through the camera parameters. SFS assumes that the surface orientation at a point $\boldsymbol{M}=[X, Y, Z]^{T}$ on a surface $\mathbf{S}$ is determined by the unit vector perpendicular to the plane tangent to $\mathbf{S}$ at $\boldsymbol{M}$. Assumed that the 3D world point is projected into the image domain at the $2 \mathrm{D}$ homogenous point $\boldsymbol{m}=[x, y, 1]^{T}$. To compute the surface normal, the partial derivatives of the $3 \mathrm{D}$ world point $\mathbf{M}$ is computed with respect to the parameters $x$ and $y$. The normal to the surface will be the cross product of the gradient vectors: $n=\mathbf{M}_{x} \times \mathbf{M}_{y}$. The partial derivatives are called surface gradients. Assuming that surface patches are homogeneous and uniformly lit by distant light sources, the brightness $\mathrm{I}(x, y)$ seen at the image plane often depends only on the orientation of the surface. This dependence on brightness of surface orientation can be represented as a func- 
tion $\mathrm{R}(x, y)$ defined on the Gaussian sphere. Thus, the SFS problem is formulated as finding a solution to the brightness equation:

$$
I(x, y)=R\left(\mathbf{M}_{x}, \mathbf{M}_{y}, \mathbf{L}\right) .
$$

where $R$ is the surface reflectance map and $\boldsymbol{L}$ is the illumination direction unit vector.

A number of algorithms were developed to estimate the illuminant direction (e.g., [41]). Some SFS approaches using perspective projection were found in the literature (e.g. $[45,46,108])$. However, most of these approaches ignore the camera extrinsic parameters, hence they cannot provide metric information about depth. In the proposed approach, the camera is calibrated and the camera parameters are used in the SFS algorithm to obtain a metric representation of the surfaces. To calibrate the camera, the relation between the 3D point $\boldsymbol{M}$ and the corresponding image coordinates $\boldsymbol{m}$ is written as the camera equation;

$$
s \boldsymbol{m}=\boldsymbol{B M}+\boldsymbol{b}
$$

where $s$ is a scalar quantity, $\mathbf{B}$ and $\mathbf{b}$ are the intrinsic and extrinsic camera matrices. These calibration matrices form a $3 \times 4$ matrix but with 11 degrees of freedom. Five parameters represent the intrinsic value while the other 6 stand for the extrinsic camera parameters [57]. The standard method of calibration is to use an object with known size and shape and extract the reference points from the object image. It can be shown that given $\mathrm{N}$ points (at least 6) in general positions, the camera can be calibrated.

The camera equation represents a line in $3 \mathrm{D}$ space corresponding to the visual ray passing through the optical center and the projected point $\boldsymbol{m}$. By finding the scalar $s$, a unique 3D point $\boldsymbol{M}$ is defined on the object by the equation:

$$
\mathbf{M}=\mathbf{B}^{-1}(s \mathbf{m}-\mathbf{b})=f(s(x, y))
$$


The surface reflectance $R($.$) becomes a function of the scalar s$ defined as:

$$
R(x, y, s)=\frac{\left(\mathbf{M}_{x} \times \mathbf{M}_{y}\right) \cdot \mathbf{L}}{\left\|\left(\mathbf{M}_{x} \times \mathbf{M}_{y}\right)\right\|}
$$

The formulation of the SFS problem becomes finding the scalar $s$ that solves the brightness equation $\mathrm{g}(x, y, \mathrm{~s})=\mathrm{I}(x, y)-\mathrm{R}(x, y, \mathrm{~s})=0$. A new variational formulation for the problem is shown below.

\section{F. The functional to be minimized}

Minimization approaches compute the solution which minimizes an energy function over the entire image. The function can involve the brightness constraint, and other constraints, such as the smoothness constraint, the integrability constraint, the gradient constraint, and the unit normal constraint. In these subsections, these constraints are briefly described.

\section{The Brightness constraint}

This is the most important constraint. The main idea is to solve for surface normals, such that when you use these normals with the given surface albedo and illumination direction. It is derived directly from the image irradiance equation. It indicates the total brightness error of the reconstructed image compared with the input image, and is given by:

$$
\varepsilon_{1}=\int_{\Omega}(I-R)^{2} d \Omega
$$

where $\Omega \in \mathbb{R}^{2}$ represents the image domain $(x, y)$ and $I$ is the image intensity. 
2. The Smoothness constraint

It is used to obtain a smooth surface that is free from discontinuities, hence the smoothness constraint can be expressed in terms of the second derivatives of surface normals to ensure surface smoothness as follows;

$$
\varepsilon_{2}=\int_{\Omega}\left(\mathbf{M}_{x x}^{T} \mathbf{M}_{x x}+\mathbf{M}_{y y}^{T} \mathbf{M}_{y y}\right) d \Omega
$$

The minimization of the above functional aims to penalize the second order gradients of the surface and hence guarantees smoothness.

3. The Integrability constraint

It ensures valid surfaces, that is, $\boldsymbol{M}_{x y}=\boldsymbol{M}_{y x}$. It can be described by:

$$
\varepsilon_{3}=\int_{\Omega}\left(\mathbf{M}_{x y}-\mathbf{M}_{y x}\right)^{T}\left(\mathbf{M}_{x y}-\mathbf{M}_{y x}\right) d \Omega
$$

4. Variational approach

There are other constraints like the unit normal and the intensity gradient constraints. The three functionals above are used in the proposed formulation. Now, the total energy will be:

$$
\varepsilon=\lambda_{1} \varepsilon_{1}+\lambda_{2} \varepsilon_{2}+\lambda_{3} \varepsilon_{3}
$$

where $\lambda_{1}, \lambda_{2}$, and $\lambda_{3}$ are real positive coefficients. 
The goal here is to compute $\mathrm{s}(x, y)$ that minimize the above energy function. Note that the above function can be written as a function in terms of the scaling factor $s$ and its derivatives:

$$
\varepsilon=\varepsilon\left(s, s_{x}, s_{y}, s_{x x}, s_{y y}, s_{x y}, s_{y x}\right)
$$

Gradient descent optimization is used with the Euler-Lagrange for computing the scalar factor as follows:

$$
\frac{\partial s}{\partial t}=-\frac{\partial \varepsilon}{\partial s}
$$

If the energy function is written as

$$
\varepsilon=\int_{\Omega} f\left(s, s_{x}, s_{y}, s_{x x}, s_{y y}, s_{x y}, s_{y x}\right) d \Omega
$$

the gradient will be as follows:

$$
\begin{aligned}
\frac{\partial \varepsilon}{\partial s} & =\frac{\partial f}{\partial s}-\frac{\partial}{\partial x}\left[\frac{\partial f}{\partial s_{x}}\right]-\frac{\partial}{\partial y}\left[\frac{\partial f}{\partial s_{y}}\right]+\frac{\partial^{2}}{\partial x^{2}}\left[\frac{\partial f}{\partial s_{x x}}\right] \\
& +\frac{\partial^{2}}{\partial y^{2}}\left[\frac{\partial f}{\partial s_{y y}}\right]+\frac{\partial^{2}}{\partial x \partial y}\left[\frac{\partial f}{\partial s_{x y}}\right]+\frac{\partial^{2}}{\partial y \partial x}\left[\frac{\partial f}{\partial s_{y x}}\right]
\end{aligned}
$$

Detailed derivations are given in the appendix.

These steps are enumerated in Algorithm 3.

\section{G. Experimental Results and Validation}




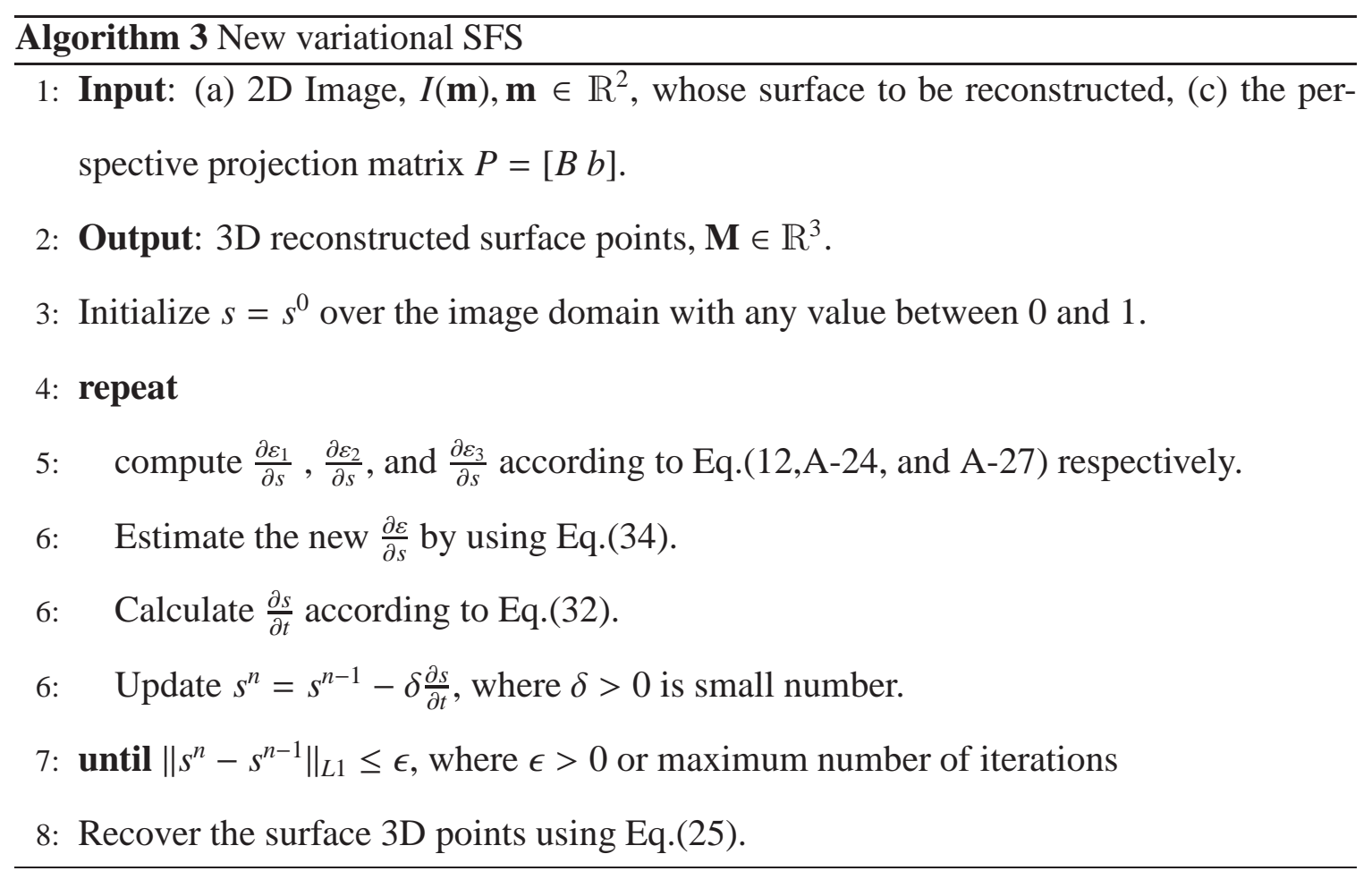




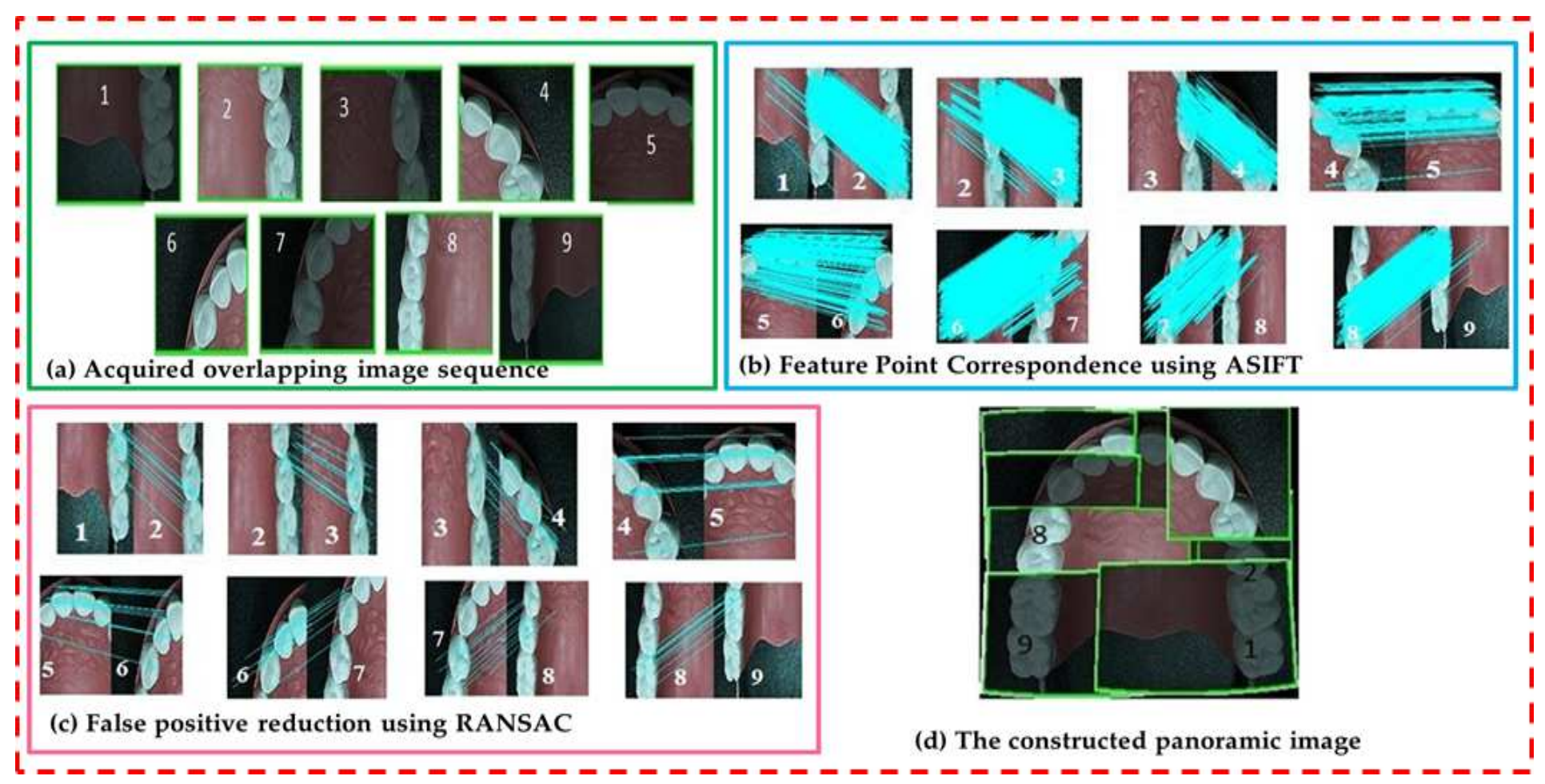

FIGURE 27: Different overlapped sub-images, results of features matching and a jaw is imaged as 9 overlapped images: (a) A sequence of overlapped images are illustrated. (b) Point correspondences as a result of the ASIFT algorithm. (c) Point correspondences are demonstrated after removing the outliers matches using RANSAC. (d) The stitching results (panoramic image) where each sub-image is boxed by a green line to show the overlap between the views. 
An intraoral camera is used to capture overlapped images of real and artificial jaws. In this stage, the camera is fixed in a certain position. The jaw is moved while the camera is fixed to image its different overlapped regions. This guarantees that all the images are calibrated. Feature points are extracted and matched for each pair of images in sequence using the ASIFT approach. Figure 27(b) demonstrates the matching process between overlapped images. The ASIFT results in a set of point matches which are almost correct except few outliers. The RANSAC as described above remove the outliers. Figure 27(c) shows the overlapped images after removing the outliers using RANSAC. This is very important in order to obtain successful registration process. The sequence of images is numbered from according to the order of taking the images. The teeth regions themselves do not have much texture information to extract features. However the edges and the contrast between the teeth and the gum plays a great role in this process. Also, for the registration approach previously described, only four points are required to estimate the transformation. This makes the resulting points more than enough for the success of registration. Using the resulting point correspondences, a projective transformation is computed and applied to the source image to get an image for stitching. Sequential images are registered together in their order and sequence. They are all put together in a bigger size image to build a panorama for the scanned jaw. Sequences of images are shown in Figure 27(a) with the stitching results illustrated in Figure 27(d). After the stitching process, some differences appear in the panoramic image (see Figure 28).

Some filtering and blending operations are carried out to remove these differences. This process is completely automatic and does not need any manual interaction. A very important note to mention here is that: Actually, if such a system is needed in clinic, the whole patient's jaw can not be imaged in one shot. The mouth environment and the specifications of the used intraoral camera allows only to image overlapped parts of the jaw. This is considered to be a strong motivation for developing the proposed system. The resulting scenes are used with the SFS technique shown above to reconstruct a 3D surface. The reconstructed surfaces include teeth details which can not be obtained by applying the SFS 

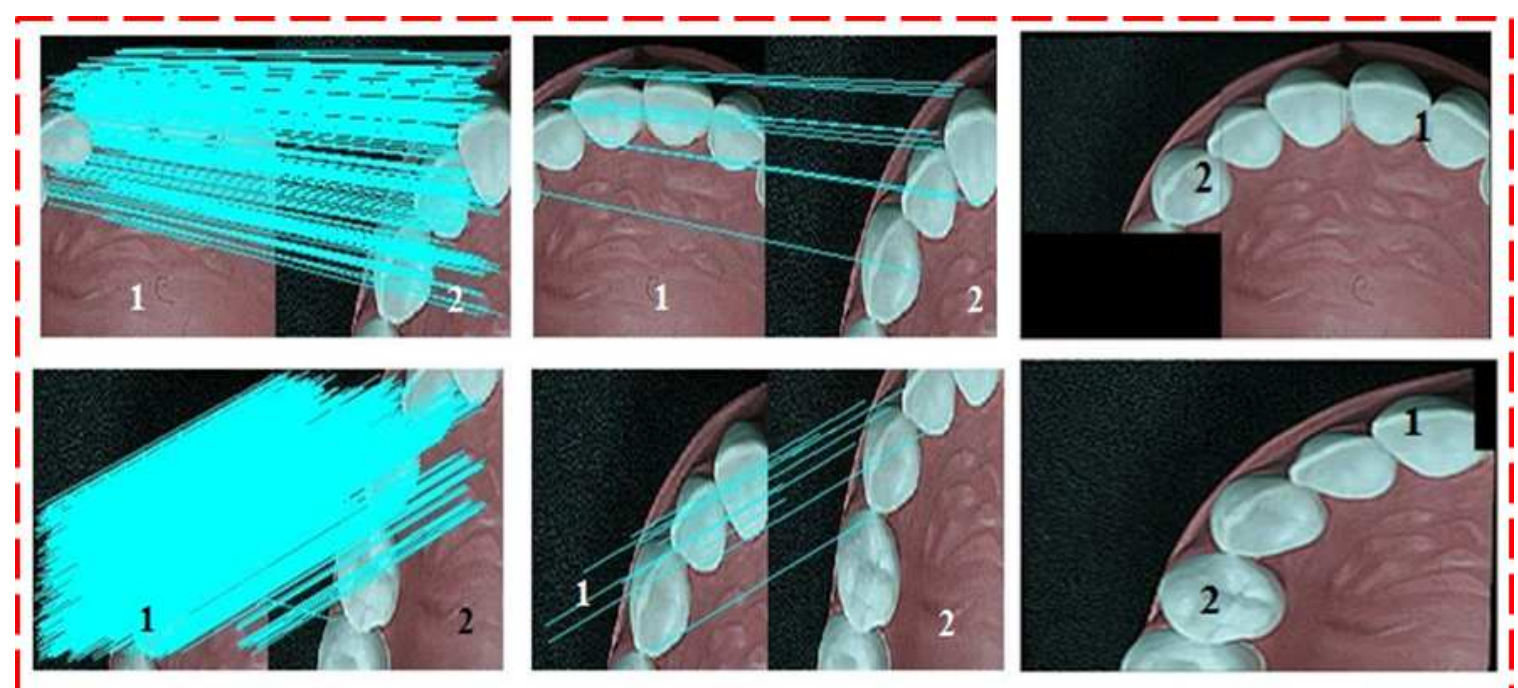

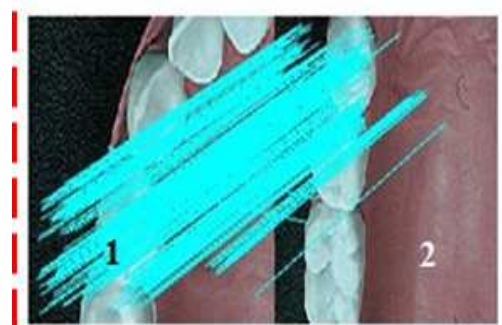

(a)

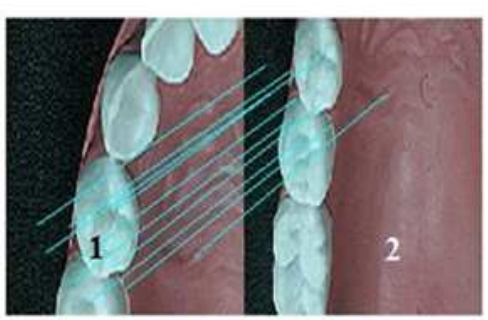

(b)

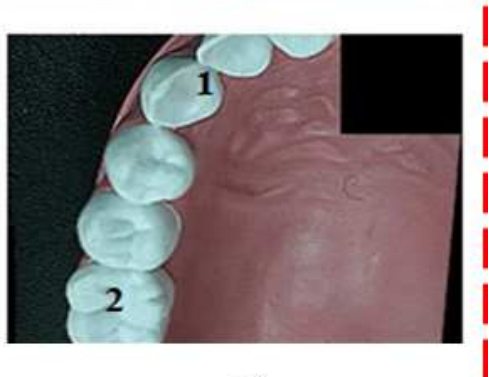

(c)

FIGURE 28: Three different examples to stitch two sub-images together: Point correspondences are demonstrated in (a) using the ASIFT algorithm. Correspondences after removing the outliers using RANSAC are shown in (b). Registration of the two overlapped images is depicted in (c). 
approach in [6] (see Figure 30). It is noticed here that the resulting surface using that approach is over-smoothed and hence upper details of teeth are smeared. Also, the transitions between teeth and gum looks unrealistic which emphasize the advantage of the technique used in this work. The whole processing time starting from taking images and ending with surface reconstruction is less than 10 minutes. The 3D surface reconstruction execution time is less than one minute. This is a big advantage for the proposed system which makes it practical and suitable for dental clinics. This will not make the patient open his mouth for a long time to get his/her jaw model. 

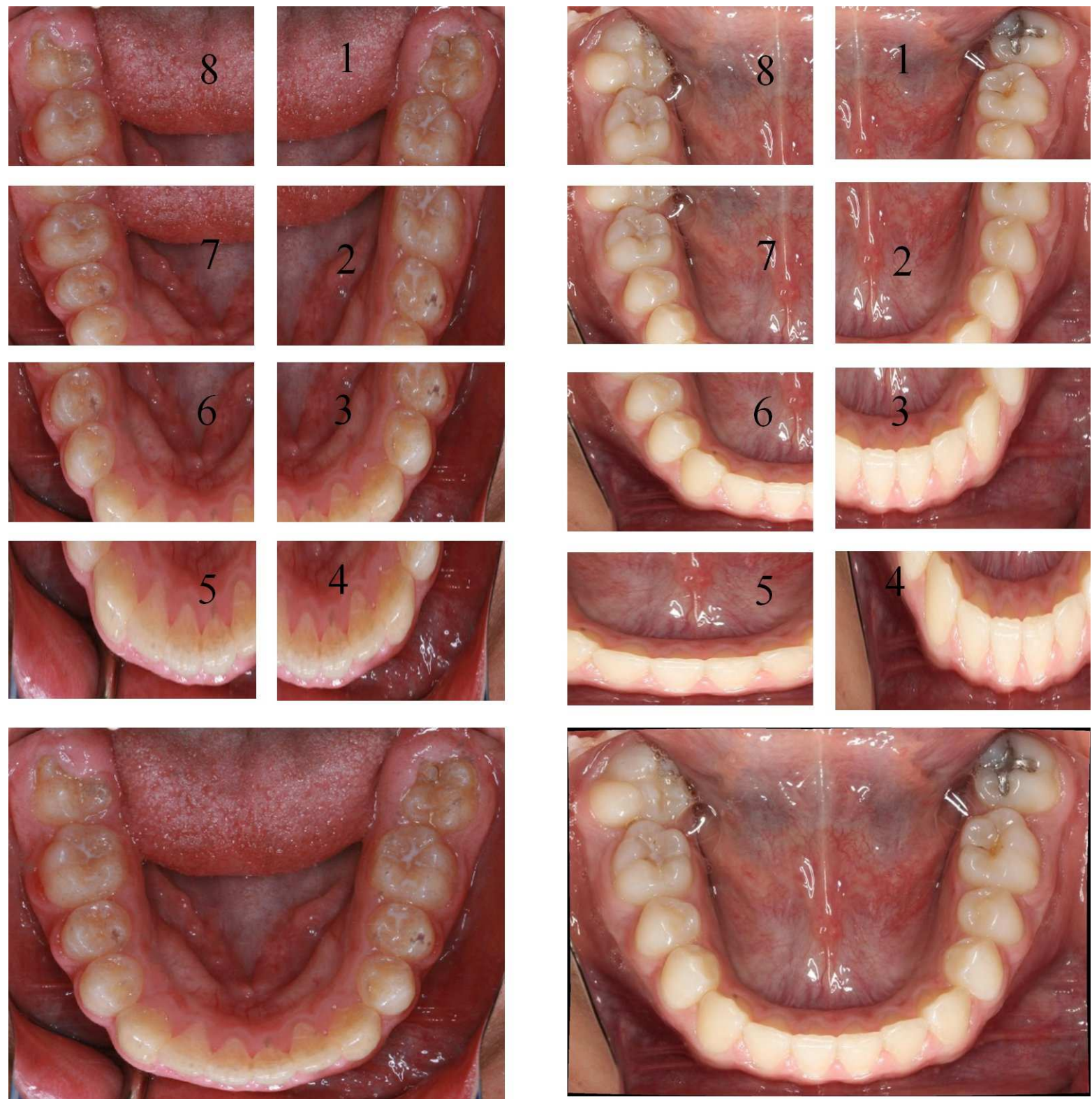

FIGURE 29: Two different real jaws sub-images panoramic reconstruction: A sequence of sub-images is given for each case from 1 to 8 while the resulting panoramic image is given at the last row. 

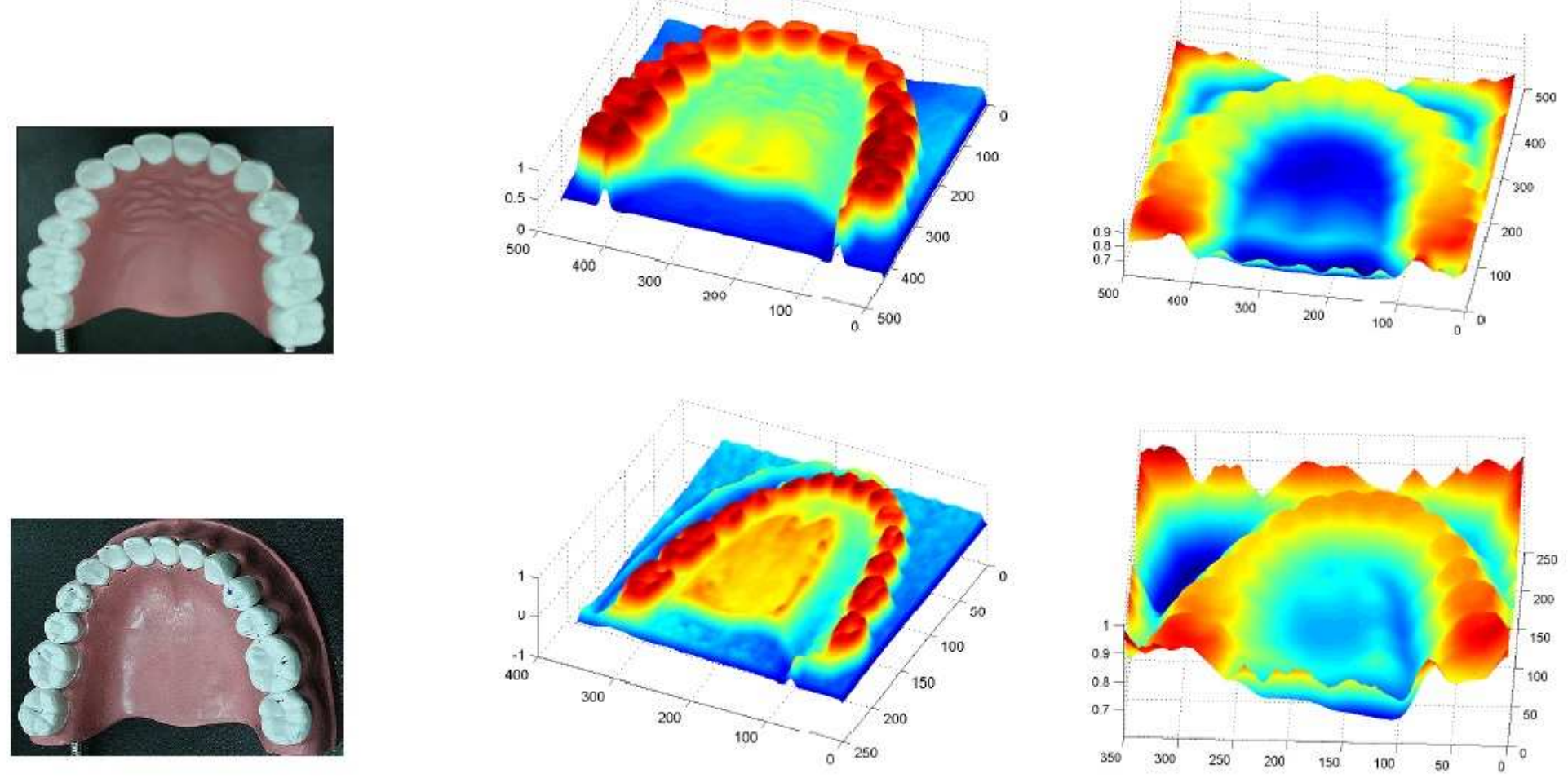

FIGURE 30: Two artificial jaws 3D surface reconstructions are demonstrated: the results of using the proposed approach are shown in the middle and the results of the technique in [6] are given in the right column. 


\section{H. Summary}

An automatic approach for 3D surface reconstruction is proposed using calibrated images with the help of the ASIFT features matching. A calibrated camera is used to capture a sequence of images for artificial jaws. Images are stitched together by estimating projective transformations for minimizing the global differences between the scenes. The registration process requires accurate point correspondences which is computed using the ASIFT approach. Camera calibration parameters are involved in the formulation of a modified version of the SFS technique to construct a 3D surface of the panoramic jaw image resulting for the stitching process. The results are preferred to other approaches in the literature since fine teeth details can be reconstructed and the resulting surface is more realistic. Also the execution time is reasonable and practical in the sense of applying the proposed system in clinic. 


\section{CHAPTER VI}

\section{REALISTIC 3D RECONSTRUCTION OF THE HUMAN TEETH USING SFS WITH SHAPE PRIORS}

This chapter, an approach is developed for realistic 3D reconstruction of the human teeth using shape from shading with statistical shape priors. The proposed work has addressed several challenges including near illumination and camera perspective projection, while taking into account the deviation from the simplifying Lambertian assumption. The Oren-Nayar reflectance model for diffuse rough surfaces is used with the roughness parameter being physically measured by an optical surface profiler. The proposed formulation exploits the shape priors as extracted from a set of training CT scans of real human teeth. Experiments provide quantitative metric results for the proposed approach.

\section{A. Introduction}

There may therefore be a demand for intraoral measurement that could be fulfilled by photogrammetry, which has been applied to the measurement of many small objects, even the measurement of dental replicas (e.g. [109]). Photogrammetry seems to offers a reduced cost technique while avoiding the need for castings. Nonetheless, intra-oral photogrammetric measurement is inherently difficult for several reasons $[18,89]$. On one end, the human jaw is not a very friendly environment for data acquisition. There are problems with lighting (effect of saliva, gum, and sources of specularity), inevitable subject's motion, and errors associated with the data acquisition sensors. On the end of image formation, the assumptions for typical shape reconstruction algorithms are hardly valid. For 
example, assumptions in previous works based on stereo (e.g.presence of distinct features or texture regions on the object in stereo images), SFS [18] (e.g.the Lambertian assumption which considers the surface in the field of view of the camera to be matte), or in space carving [19] (e.g.the photo consistency) are rarely valid in practice.

In the context of tooth reconstruction from intra-oral images, the SFS technique offers several advantages. It provides rather more detailed and accurate representation about the shape of the tooth crowns [18]. It requires only one camera position within the cramped confines of the mouth. Cost-wise, it is cheaper, because it simply requires a single camera and light source.

This work, aims to address the aforementioned issues related to the acquisition setup and the shape reconstruction approach. A flexible and convenient, both to the dentist and the patient, acquisition setup has been developed that consists of a small wireless intraoral camera with a built-in bright light source to acquire $2 \mathrm{D}$ images of the teeth. The camera has 1/4 Sony CCD, and an image resolution of 2.0 mega pixels (see Figure 1).

From the point of view of shape reconstruction, A more realistic formulation of SFS is introduced by considering all the components of the problem, namely: the camera, the light source, and the surface reflectance. The camera is modeled by perspective projection, which is more practical in this case as the teeth are typically close to the camera. The light source is assumed to be located at the optical center of the camera. Under this near-illumination imaging the attenuation of illumination is taken into account due to the distance between the light source and the surface, which helps to deal with the concave/convex ambiguity in SFS $[9,22]$. As the Lambertian reflectance is rather oversimplified to model the real nature of the human teeth surface, the Oren-Nayar model is used [15] which carefully accounts for the scattering of light caused by the teeth surface roughness. In the proposed approach, this surface roughness is even physically measured from real human teeth using an optical surface profiler. 
In addition, to further improve the accuracy and practicality of results, 3D shape priors in SFS formulation are incorporated. This strategy is motivated by the fact that humans rely on strong prior information about the 3D world around us in order to perceive 3D shape information. It thus becomes natural to think of ways to incorporate this kind of information into the reconstruction process done by computers. Such information is statistically extracted from training 3D models of the human teeth. This can serve in several aspects, e.g., to improve reconstruction accuracy, solve problems caused by occlusion (e.g., because of the tongue), specularity and albedo changes, and/or make up for the lack of sufficient, detailed view of the human tooth.

The proposed method of incorporating 3D shape priors in SFS can be linked to the so-called statistical SFS methods in literature, e.g. [110-112], all of which have been developed in the context of 3D face reconstruction. In particular, the same idea in $[110,111]$ of constructing a 3D prior model based on a low-dimensional parametrization of a training set is employed. This is achieved by applying principal component analysis (PCA). In addition to the different application domain (tooth reconstruction in the proposed case), this work here conveys other different and novel aspects. While those existing SFS methods [110-112] have assumed Lambertian model and orthographic projection, the more practical Oren-Nayar model with perspective projection is moved further ahead. While some of these methods (e.g., [112]) use harmonic representations of lighting to account for non-known illumination source, and may require a separate model for the face albedo, the proposed approach in that regard is simpler by the design of the acquisition setup, where the light source position is known. Therefore, in summary, the proposed approach utilizing 3D shape priors as well as the more realistic assumptions of the Oren-Nayar reflectance model, perspective projection and near light source with intensity attenuation, to the best of knowledge, has not been done before in literature. Several successful experimental results of the proposed approach are reported on real teeth with specularity and other challenging conditions. 


\section{B. Shape Priors}

A shape model is constructed from a training set of 3D model of the same human tooth type. To be able to do this, several real invitro teeth of the same type (maxillary deciduous, maxillary molars, mandibular molars, and mandibular third molars) from adult subjects with different races, gender and ages are fixed over wax. A Cone-beam CT (KODAK 9000 3D Extraoral) scanner at a resolution of $0.2 \mathrm{~mm}$ is then used to scan the wax and teeth. At least 30 teeth of each type have been collected. The root part of each tooth is manually taken out from the CT scan, and the visible 3D tooth surface is segmented out.

Furthermore, the surface roughness $\sigma$ is estimated of the tooth surfaces which is needed for the Oren-Nayar diffuse reflection model. This is done with the help of a 3D optical surface profiler (NewView 700s from Zygo company), which is based on Scanning White-Light Interferometry technology that offers fast, non-contact, high-accuracy 3D metrology of micro surface features.

Afterwards, the 3D surfaces for each tooth type are rigidly aligned to remove any variations in shape due to pose differences. The first surface of the training set is used as the reference to which the remaining surfaces are aligned. The alignment is carried out using an iterative closest point (ICP-based) rigid registration algorithm [85] using the Hausdorff distance between corresponding points.

However before either case, the input brightness image of a human tooth whose 3D surface to be reconstructed must be aligned first with the constructed shape model. This is successfully achieved by a rigid 2D image registration between the input image and a reference image for the constructed shape model using the maximization of mutual information [113]. Figure 31 shows one sample input image for a tooth, the reference image for a constructed shape model, and the input image after alignment.

To build the shape model, each 3D tooth surface is converted to a height map, then 

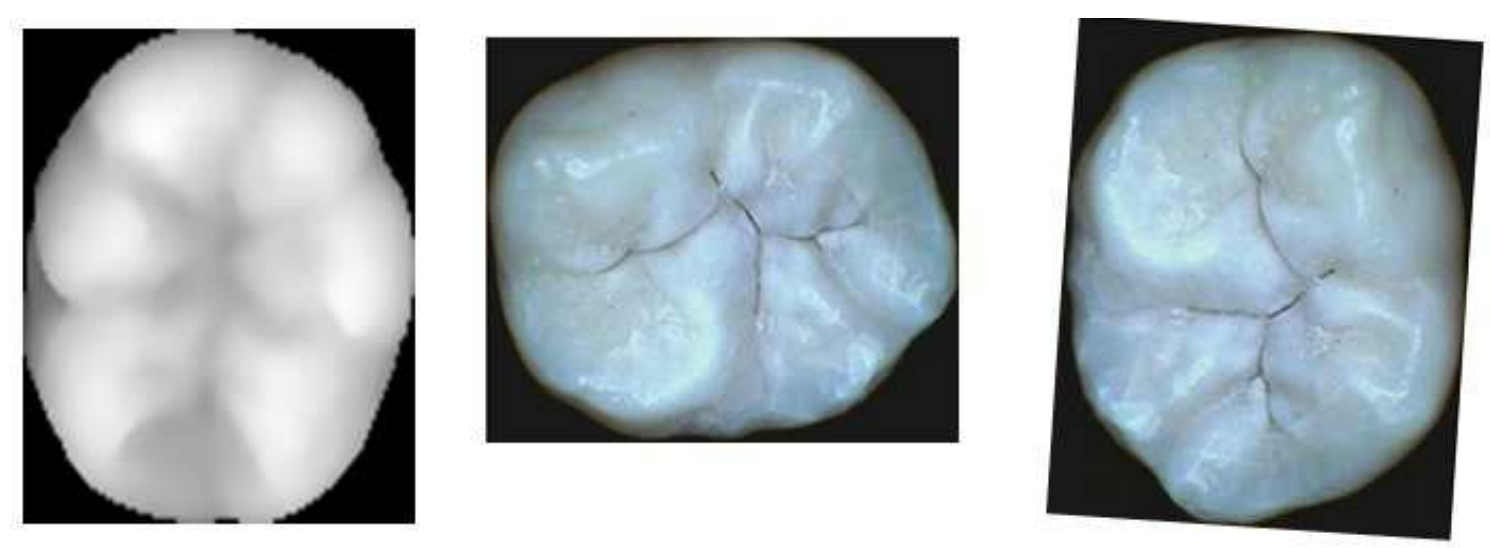

FIGURE 31: Aligning an input image with the reference shape model. (Left) Reference image corresponding to the constructed shape model. (Middle) Sample input test image for a tooth. (Right) The test image after being aligned with the reference image.

principal component analysis (PCA) is applied to capture the main variations of the coaligned height maps:

$$
u(\mathbf{x})=\bar{u}+\sum_{i=1}^{k} w_{i} u_{i}(\mathbf{x})
$$

where $\bar{u}$ is the mean height map (mean tooth shape), see Figure $32, u_{i}(\mathbf{x})$ is the $i-t h$ orthogonal mode of variation in the shape (also called eigenteeth), and $\mathbf{w}=\left\{w_{i}\right\}$ is called the vector of eigen coefficients or the shape vector. Only k principal components are considered in the sum, where k should be chosen large enough to be able to capture the prominent shape variations present in the human teeth.

Equation (35) is used as explicit representation of teeth shape. Therefore, by varying $\mathbf{w}$, vary $u$. Note that the shape variability allowed in this representation is restricted to the variability given by the eigenteeth.

\section{Shape from Shading with Shape Priors}



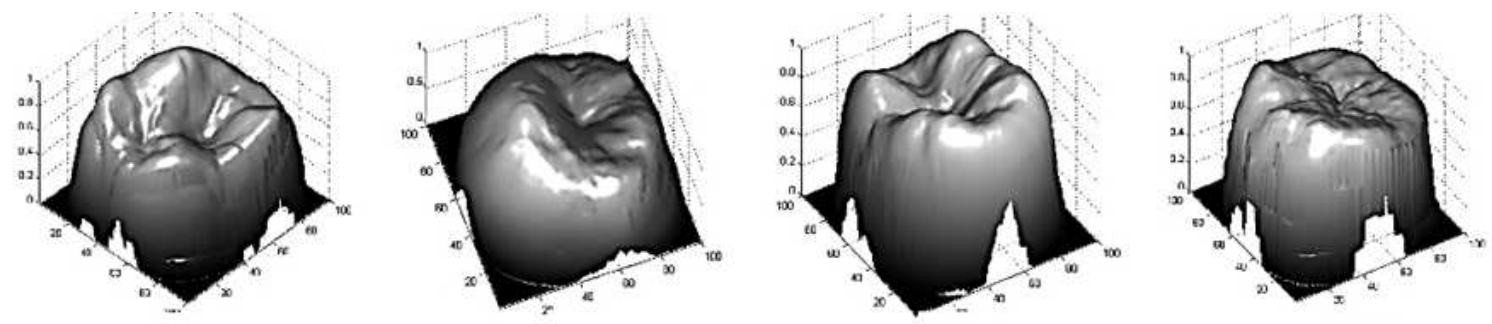

FIGURE 32: The mean shapes in the constructed database of human teeth of various types: maxillary deciduous teeth, maxillary molars, mandibular molars and mandibular third molars, respectively.

Having built the prior shape model, it is ready now to embed it in the SFS framework to guide the solution. Starting the simple case of Lambertian surface, a distant directional light source and orthographic projection are used to demonstrate the proposed formulation. The concern then shifts to the more realistic SFS formulation considering the Oren-Nayar diffuse reflection model with a near light source and perspective camera.

However before either cases, the input brightness image of a human tooth whose 3D surface to be reconstructed must be aligned first with the constructed shape model. This is successfully achieved by a rigid 2D image registration between the input image and a reference image for the constructed shape model using the maximization of mutual information [113].

\section{Simple Lambertian Case}

The simplest imaging model is obtained when the camera performs an orthographic projection of a surface that has Lambertian reflectance and illuminated by a point light source located far away from the surface. The idea here is to solve for the height map $u(\mathbf{x})$ that minimizes the energy functional: 


$$
\varepsilon_{L}=\int_{\Omega}\left(I(\mathbf{x})-R(u(\mathbf{x}))^{2} d \mathbf{x}=\int_{\Omega}\left(I(\mathbf{x})-\frac{\hat{s} \cdot(\nabla u,-1)}{\sqrt{1+|\nabla u|^{2}}}\right)^{2} d \mathbf{x}\right.
$$

where $\Omega \subseteq \mathbb{R}^{2}$ represents the image spatial domain, and $I$ is the image intensity.

Solving this last optimization problem is rather difficult and often requires imposing some regularization (e.g., smoothness of height map) [17]. Nevertheless, utilizing the prior shape model (35) in terms of the weighted sum of eigen vectors, the task is reduced to searching for the weights $w_{i}$ that minimize the energy function (not functional anymore)

$$
\varepsilon_{L}=\int_{\Omega}\left(I(\mathbf{x})-\frac{\hat{s} \cdot\left(\sum_{i=1}^{k} w_{i}\left[\frac{\partial u_{i}(\mathbf{x})}{\partial x} \frac{\partial u_{i}(\mathbf{x})}{\partial y}\right],-1\right)}{\sqrt{1+\left(\sum_{i=1}^{k} w_{i} \frac{\partial u_{i}(\mathbf{x})}{\partial x}\right)^{2}+\left(\sum_{i=1}^{k} w_{i} \frac{\partial u_{i}(\mathbf{x})}{\partial y}\right)^{2}}}\right)^{2} d \mathbf{x} .
$$

One can readily see a quick advantage in the above optimization problem (37), where the solution search space is shrinked into a finite number $(k)$ of weights. To find this solution, one can easily use a gradient descent minimization routine, where the weights $\left\{w_{i}, i=1, \cdots, k\right\}$ are evolved according to

$$
\frac{\partial w_{i}}{\partial t}=-\eta \frac{\partial \varepsilon_{L}}{\partial w_{i}}
$$

where $\eta$ is a positive learning constant. The required partial derivatives of the energy function $\varepsilon_{L}$ with respect to the weights $\left\{w_{i}\right\}$ are readily obtained as

$$
\begin{array}{r}
\frac{\partial \varepsilon_{L}}{\partial w_{i}}=\int_{\Omega} 2\left(I(\mathbf{x})+\frac{v}{\sqrt{1+|\nabla u|^{2}}}\right) \times \\
{\left[\frac{\left(1+|\nabla u|^{2}\right)\left(-s_{1} \frac{\partial u_{i}(\mathbf{x})}{\partial x}-s_{2} \frac{\partial u_{i}(\mathbf{x})}{\partial y}\right)-v p}{\left(1+|\nabla u|^{2}\right)^{1.5}}\right] d \mathbf{x},}
\end{array}
$$


where

$$
\begin{array}{r}
v=\left(-s_{1} \sum_{i=1}^{k} w_{i} \frac{\partial u_{i}(\mathbf{x})}{\partial x}-s_{2} \sum_{i=1}^{k} w_{i} \frac{\partial u_{i}(\mathbf{x})}{\partial x}+s_{3}\right) \\
p=\left(\sum_{i=1}^{k} w_{i} \frac{\partial u_{i}(\mathbf{x})}{\partial x}\right) \frac{\partial u_{i}(\mathbf{x})}{\partial x}+\left(\sum_{i=1}^{k} w_{i} \frac{\partial u_{i}(\mathbf{x})}{\partial y}\right) \frac{\partial u_{i}(\mathbf{x})}{\partial y} .
\end{array}
$$

In experimentation, the evolution commences from $\left\{w_{i}=0.2, i=1, \cdots, k\right\}$ at $t=$ 0 , and convergence is typically attained within rather few iterations. From the obtained weights, the height map is recovered via (35) in order to reconstruct the surface of the tooth.

\section{Realistic Oren-Nayar case}

In this case the Oren-Nayar model is used for the surface reflectance. The camera has a perspective projection and the light source is assumed to be located at the optical center of the camera, with its intensity being attenuated with squared distance.

The energy functional to be minimized in this case is

$$
\varepsilon_{O N}=\int_{\Omega}\left(I(\mathbf{x})-R(u(\mathbf{x}))^{2} d \mathbf{x},\right.
$$

where

$$
R(u(\mathbf{x}))=\frac{\rho}{\pi f^{2} u^{2}(\mathbf{x})} L_{i}\left(A(\hat{s} \cdot \hat{n})+B\left(1-(\hat{s} \cdot \hat{n})^{2}\right)\right) .
$$

It is a common practice [17] to drop the constants $\left(\rho, L_{i}, f\right)$ in (41), and alternatively normalize the input intensity image $I$. From the definitions of the perspective camera in Section II.F.2 and Figure 14, one can easily obtain

$$
\hat{s} \cdot \hat{n}=\frac{f}{\sqrt{x^{2}+y^{2}+f^{2}}} u(\mathbf{x}) .
$$


The goal here is to find the solution of the optimization problem

$$
\hat{w}_{i}=\arg \min _{w_{i}} \varepsilon_{O N}, i=1, \cdots, k,
$$

which easily solved using a gradient descent minimization routine, where the weights $\left\{w_{i}, i=1, \cdots, k\right\}$ are evolved analogously to (38). The required partial derivatives of the energy function $\varepsilon_{O N}$ with respect to the weights $\left\{w_{i}\right\}$ are readily obtained as

$$
\frac{\partial \varepsilon_{O N}}{\partial w_{i}}=\int_{\Omega} 2[I(\mathbf{x})-R(u(\mathbf{x}))]\left(-\frac{\partial R(u(\mathbf{x}))}{\partial w_{i}}\right) d \mathbf{x}
$$

where

$$
\frac{\partial R(u(\mathbf{x}))}{\partial w_{i}}=\frac{\rho L_{i}}{\pi}\left[\frac{-A u_{i}(\mathbf{x})}{f \sqrt{x^{2}+y^{2}+f^{2}} u^{2}(\mathbf{x})}-2 \frac{B u_{i}(\mathbf{x})}{f^{2} u^{3}(\mathbf{x})}\right]
$$

It is interesting to note here that the update equation of the weights in this case does not depend on the the spatial derivatives of the shape eigenvectors $\left(\frac{\partial u_{i}}{\partial x}, \frac{\partial u_{i}}{\partial y}\right.$, or $\left.\nabla u\right)$. This is in contrast to the weight update equation for the simpler Lambertian case (see (39)). This leads to more noise-robust evolution of the weights in the more realistic case and faster convergence (about only $60 \%$ of the iterations of the simple Lambertian case is typically needed here in the experiments).

\section{Experimental Results}

In order to evaluate the performance of the proposed approach, several experiments are carried out on real human teeth, other than the ones used for constructing the shape prior model. The accuracy of the tooth reconstruction is assessed by comparing it to the 3D ground-truth surface as obtained from CT scan. For the proposed approach, the simple Lambertian case (Section VI.C.1) and the more realistic case (Section VI.C.2) are applied both in order to assess the gain out of the latter formulation. 
TABLE 4: Summary of approaches under experimental comparison.

\begin{tabular}{|c|c|c|c|c|c|}
\hline & Camera & Light source & Reflectance & Priors & Reference \\
\hline AA & orthographic & at infinity & Lambertain & No & {$[78,114]$} \\
BB & perspective & at optical center & Oren-Nayer & No & {$[9,12]$} \\
CC & orthographic & at infinity & Lambertain & Yes & new \\
DD & perspective & at optical center & Oren-Nayer & Yes & new \\
\hline
\end{tabular}

The proposed algorithm is compared with two SFS algorithms: The first is a standard SFS algorithm $[78,114]$ assuming typical assumptions of Lambertian surface, orthographic projection and distant directional light source. The other algorithm is a more recent SFS algorithm $[9,12]$ based on viscosity solutions for Hamilton-Jacobi type formulation of the image irradiance equation. This algorithm was originally developed in [9] for the Lambertian case assuming a perspective camera projection and a light source at the camera optical center. Later [12] it has been extended to the Oren-Nayar reflectance model. It does not use any prior information in its SFS formulation. Table 4 summarizes the key differences between the proposed approaches (CC and DD) and the others approaches (AA and $\mathrm{BB})$.

Sample results from the approaches under evaluation are demonstrated in Figure 33. Figure 33(first row) shows the 2D input images for four different teeth models (maxillary deciduous, maxillary molar, mandibular molar, and mandibular third molar), while Figure 33(second row) shows the corresponding ground-truth tooth surface as obtained from CT scans. The outputs from Algorithm AA, shown in Figure 33(third row), are rather bumpy with too many peaks as the algorithm fails to handle the inevitable specularity due to the tooth surface characteristics and the near-illumination setup. Rather better reconstructions are provided by Algorithm BB, see Figure 33(fourth row). However the algorithm is not able to recover the geometrical details of the occlusal surface when compared to the proposed algorithms CC (Figure 33(fifth row)) and DD (Figure 33(last row)). The shape priors have indeed guided the SFS formulation to recover more details of the tooth crowns 
overcoming the challenges caused by the scattered specular spots throughout the tooth surface. Clearly, the more realistic formulation (algorithm DD) has shown the most success in that regard. Beneath each reconstruction is the root-mean-square $(R M S)$ error between the reconstruction and the ground-truth after performing 3D rigid registration. Consistently, the proposed algorithms (CC and DD) provide smaller RMS errors, with Algorithm DD having the lowest error rate (almost as low as one half of those for Algorithms AA and BB). This emphasizes the role of incorporating prior information in the process of shape recovery.

To further demonstrate the gain out of the shape priors, another experiment is performed on real human teeth with fillings that cover significant parts of the teeth, see Figure 34 (first row). Figure 34 (second row) shows the corresponding surface reconstructions by the proposed approach (Algorithm DD). Note that the teeth are successfully and completely reconstructed, in spite of the tooth filling regions with different colors and albedo characteristics in the input images. This notable outcome of the proposed approach becomes more evident when compared to the tooth reconstruction by Algorithm BB in Figure 34 (third row). The filling regions gave rise to significant valleys and grooves in Algorithm $\mathrm{BB}$ results.

\section{E. Summary}

This chapter, focused on realistic 3D reconstruction of the human teeth using shape from shading with statistical shape priors. The work has addressed several challenges related to the image formation process including near illumination, perspective projection, while taking into account the deviation from the simplifying Lambertian assumption. The Oren-Nayar reflectance model is used for diffuse rough surfaces with roughness parameter being physically measured by an optical surface profiler. The proposed formulation exploits the shape priors as extracted from a set of training CT scans of real human teeth. 


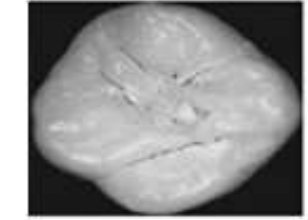

Maxillary deciduous
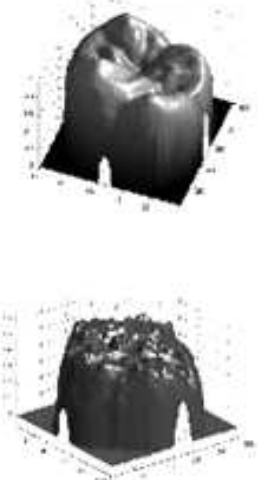

$R M S=1.2561 \mathrm{~mm}$

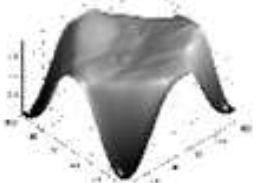

$R M S=1.0588 \mathrm{~mm}$

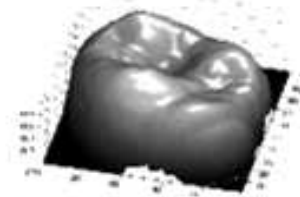

$R M S=0.5596 \mathrm{~mm}$

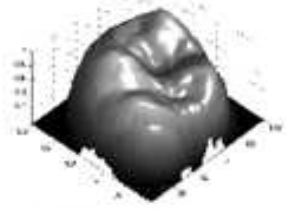

$R M S=0.5021 \mathrm{~mm}$

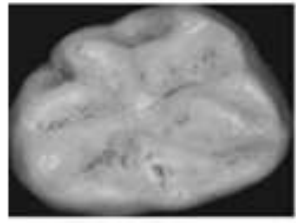

Maxillary molar
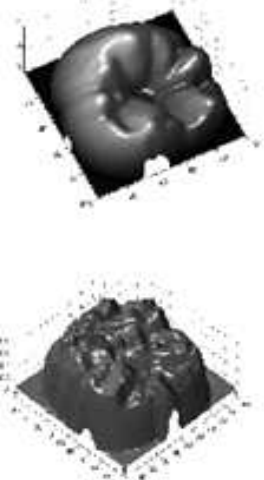

$R M S=0.8542 \mathrm{~mm}$

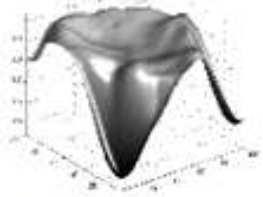

$R M S=0.8384 \mathrm{~mm}$

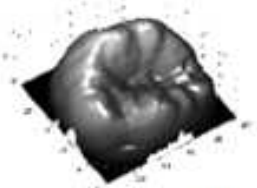

$R M S=0.5384 \mathrm{~mm}$

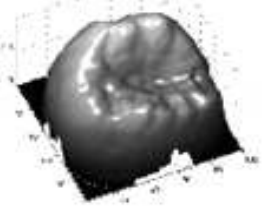

$R M S=0.5095 \mathrm{~mm}$

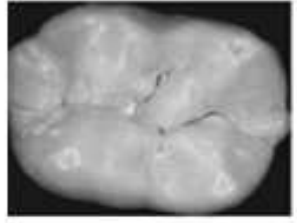

Mandibular molar
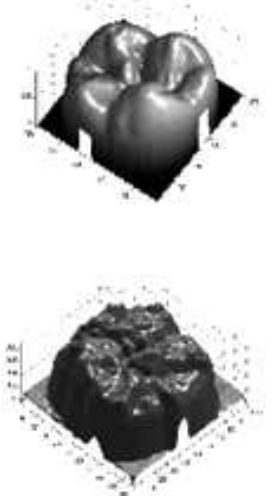

$R M S=1.5225 \mathrm{~mm}$

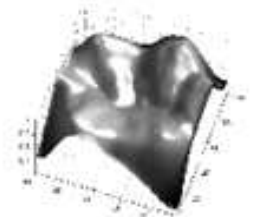

$R M S=1.0206 \mathrm{~mm}$

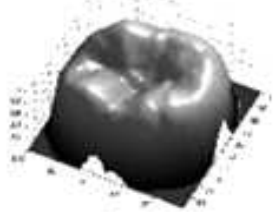

RMS $=0.4600 \mathrm{~mm}$

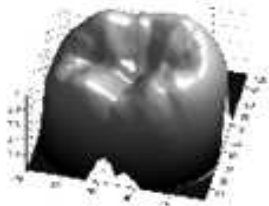

$R M S=0.4500 \mathrm{~mm}$

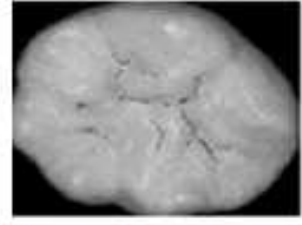

Mandibular $3^{\text {rd }}$ molar
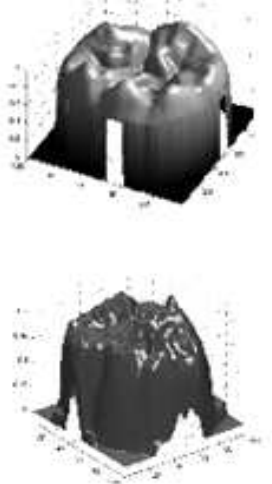

$R M S=0.9854 \mathrm{~mm}$

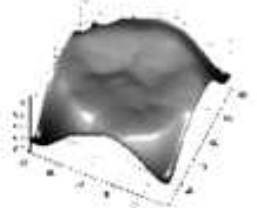

$R M S=1.0793 \mathrm{~mm}$

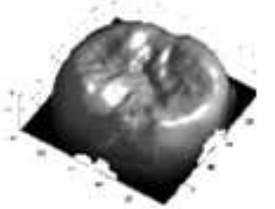

$R M S=0.5078 \mathrm{~mm}$

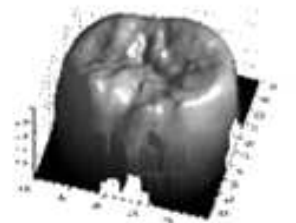

$R M S=0.4273 \mathrm{~mm}$

FIGURE 33: Tooth reconstruction from four different algorithms. First row: image acquired by the intraoral camera. Second row: ground-truth occlusal surface generated from a CT scan of the tooth. Third row: reconstruction using Algorithm AA. Fourth row: reconstruction using Algorithm BB. Fifth row: reconstruction using the proposed method (Algorithm CC). Last row: reconstruction using the proposed method (Algorithm DD). Beneath each reconstruction is the root-mean-square $(R M S)$ error when compared to the ground-truth surface from CT. 

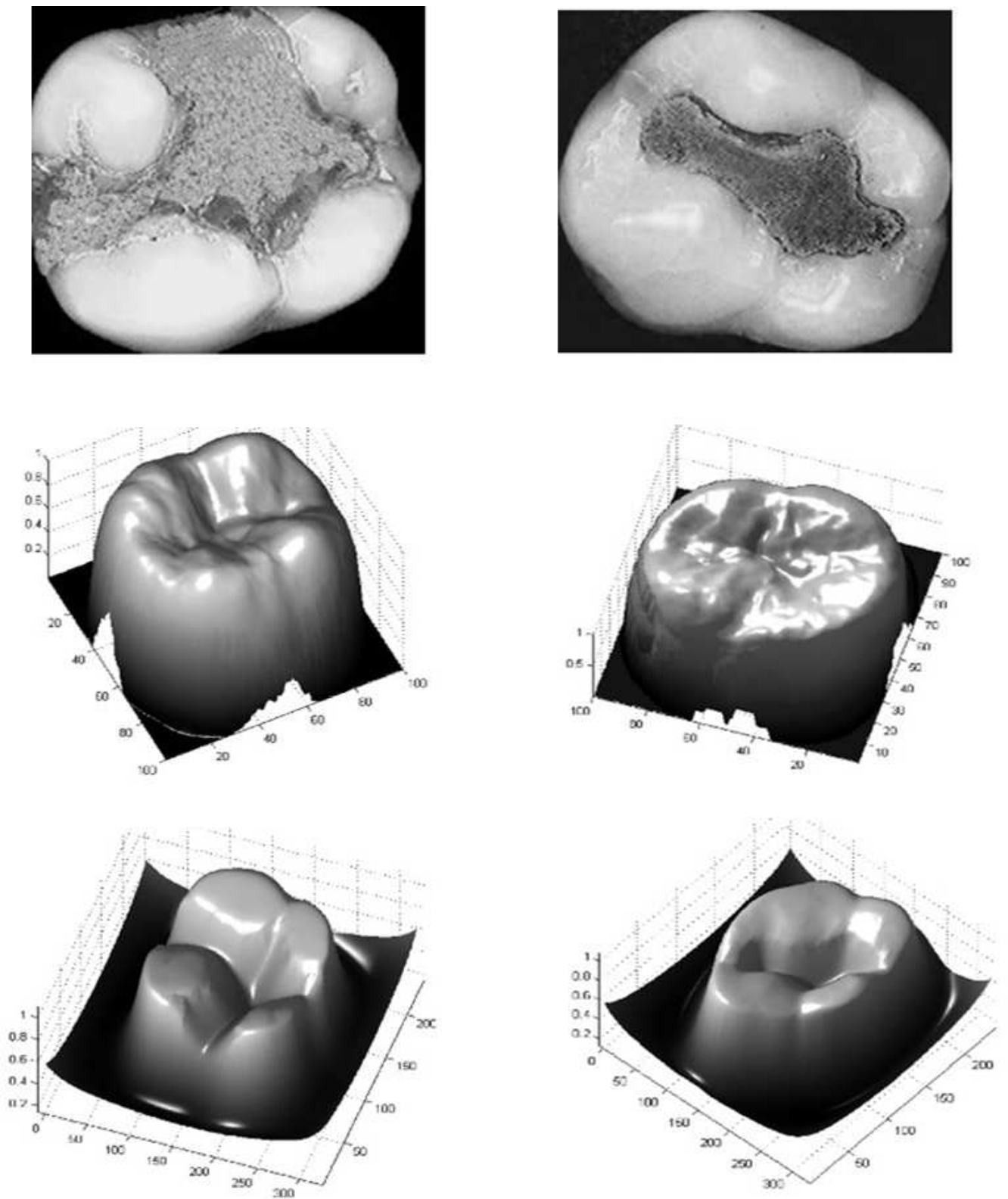

FIGURE 34: Experiment on teeth with significant fillings. First row: input test image. Second row: reconstruction by the proposed algorithm (Algorithm DD). Third row: reconstruction by Algorithm BB. 
This allows the reconstruction of a tooth with shape variations consistent with the training model examples. The proposed approach utilizing 3D shape priors as well as those more realistic assumptions of the Oren-Nayar reflectance model, perspective projection and near light source with intensity attenuation, to the best of knowledge, has not been done before in literature.

Experimental results have stressed the importance of invoking the shape priors and realistic object characteristics into surface reconstruction. The proposed approach has been able to recover rich geometric details of tooth occlusal surfaces. Furthermore, shape priors have helped in handling specular regions in tooth surface due to saliva and enamel reflectance. In particular, on the average, the proposed approach reduces the error metric by $0.5-0.65 \mathrm{~mm}$ compared to well-known existing SFS approaches. It is important to stress here that in this application domain, fractions of a millimeter improvements are considered significant for dental-related applications such as tooth implant and surface analysis. The proposed approach have successfully reconstructed teeth with challenging conditions, such as scattered specular spots and significant changes in color and albedo characteristics. 


\section{CHAPTER VII}

\section{D-PRINCIPLE COMPONENTS ANALYSIS SHAPE MODELS}

In this chapter, the current algorithms that were presented in the previous Chapter (VI) are improved. The approached developed in this chapter reconstructs the teeth from single image shading with 2D-Principal Components Analysis (PCA) shape priors that have more sophisticated reflectance model. The Oren-Nayar-Wolff model was used for modeling the surface reflectance. This formulation uses shape priors as retrieved from a set of training CT scans of real human teeth. The experiments show promising quantitative results, which builds the infrastructure for having an optical based approach that accounts for inexpensive and radiationless human tooth reconstruction.

\section{A. Introduction}

The two systems that showed most promise in the last few years, the iTero (Cadance)(see Figure $35^{9}$ ) and Lava(see Figure $36^{10}$ ). The Probes in both systems are bulky and requiring multiple scans to get full coverage of the oral cavity. The Lava system requires the use of a visible powder to get good registration, and has problems with depth of field. The iTero has a heavier probe and can only capture one tooth at a time, requiring five views of each tooth. Blood and saliva causes additional inaccuracies with both systems.

In Chapter VI, a 3D reconstruction of the human teeth is presented using SFS with shape priors, this work lacking in the following aspects: (1) They assumed Oren-Nayar

\footnotetext{
${ }^{9}$ http://www.sheffermanortho.com/news.php.

${ }^{10}$ http : //www.medgadget.com/2008/07/3m_espe_lava_oral_scanner_gets_ideabronze.html.
} 


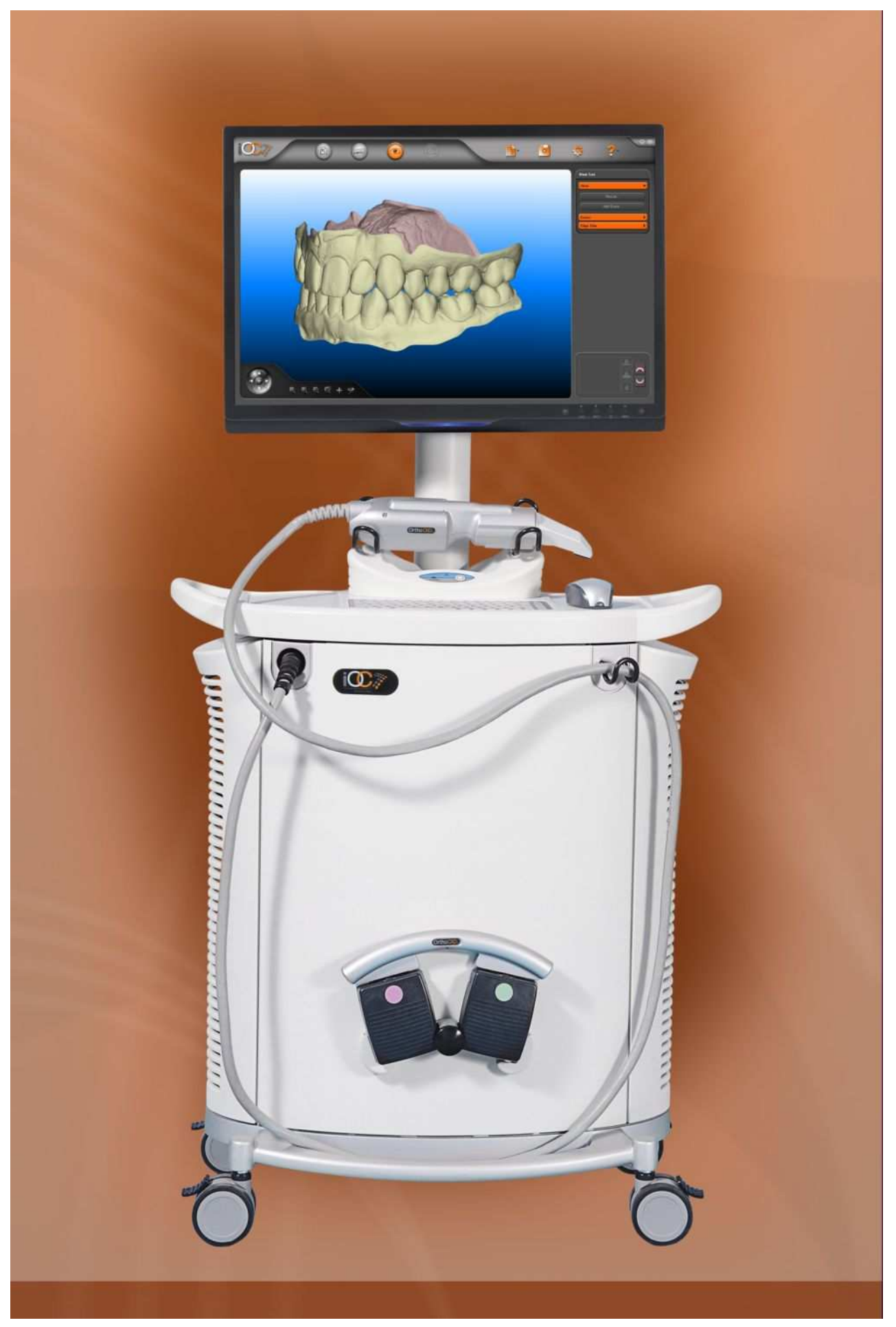

FIGURE 35: iTero commercial dental scanning system. 


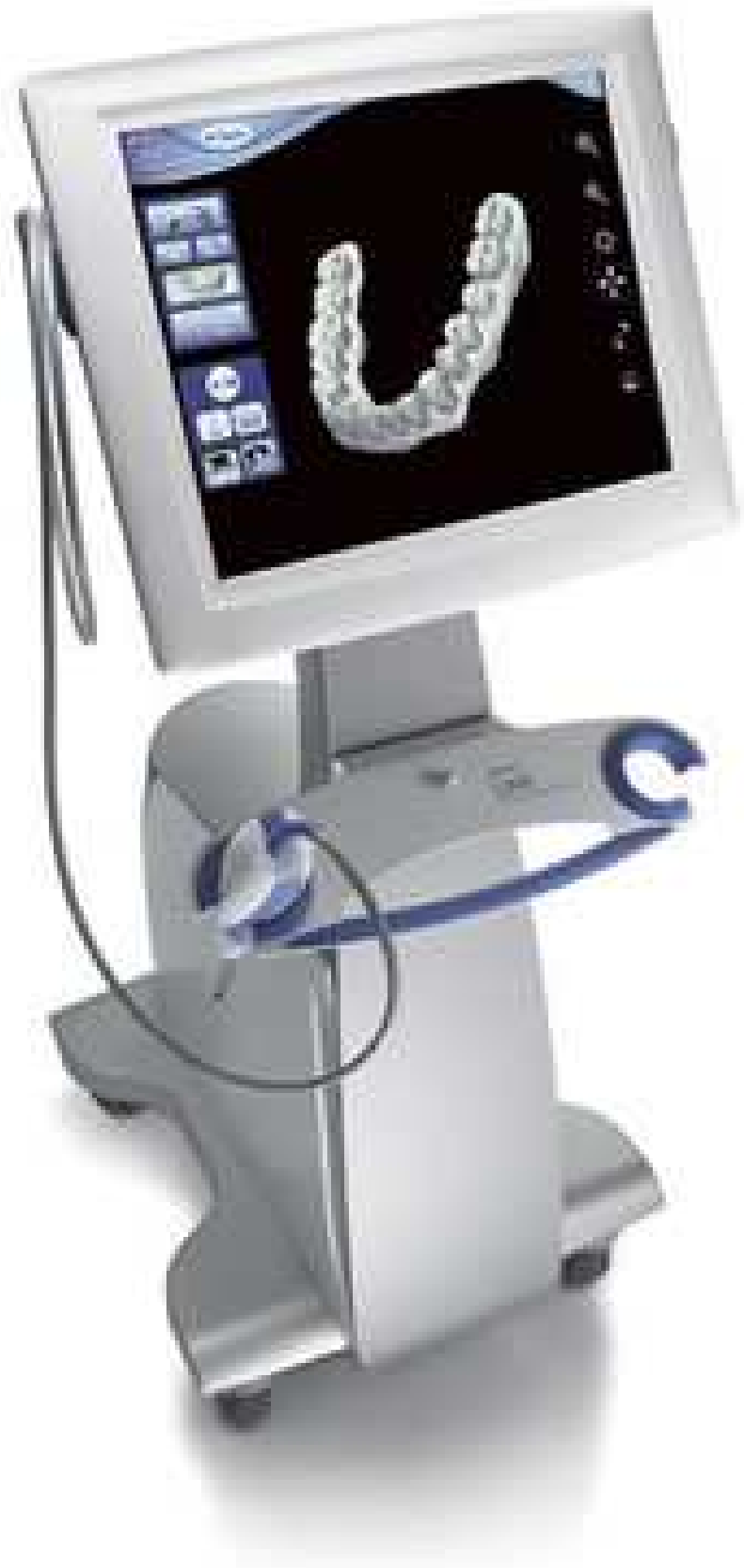

FIGURE 36: Lava commercial dental scanning system. 
model for tooth surface reflectance. Nonetheless, tooth surface is rough and wet. (2) Shape prior information was constructing using 2D-Principal Component Analysis where PCA is time consuming to determine the corresponding eigenvectors.

PCA is one of a family of techniques for taking high-dimensional data, and using the dependencies between the variables to represent it in a more tractable, lower-dimensional form, without losing too much information. PCA is one of the simplest and most robust ways of doing such dimensionality reduction. It is also one of the oldest, and has been rediscovered many times in many elds, so it is also known as the Karhunen-Love transformation, the Hotelling transformation, the method of empirical orthogonal functions, and singular value decomposition.

In this chapter, the goal is to achieve further improvement in the accuracy of the human tooth reconstruction approach in Chapter VI [21]. The contribution in this chapter are two-fold. First, the 2D-PCA is used to build the shape priors instead of the conventional PCA. The 2D-PCA offers two important advantages [115-117]: It is easier to evaluate the covariance matrix accurately since its size is much smaller. In addition, less time is required to determine the corresponding eigenvectors [115-117]. Second, the modified Oren-Nayar-Wolff reflectance model [92] is presumed in place of the Oren-Nayar model assumed in Chapter VI, where the tooth surface is rough and wet, giving rise to Fresnel reflection due to different refractive indices of the saliva and the tooth material. The tooth surface roughness is physically measured using an optical surface profiler(see Figure 22). The proposed CCD based intraoral camera is a factor of 10 less expensive, allowing it to be affordable for most dental and orthodontist offices. The system is 20 times lighter, which reduces fatigue of the operator enormously. Another major difference in the proposed system, the output file of the proposed system will be readily available as an STL file instead of locked in a proprietary format such as iTero and Lava have done.

\section{B. Image Irradiance Equation}


One good model for tooth surface reflectance is the Oren-Nayar-Wolff models which work well for the retro-reflection case [94]. Figure 22 shows microscopic view of the occlusal surface height variations which proves that micro-facet reflectance models are suitable for the tooth surface. Assume the camera is modelled with a perspective projection. According to the presented data acquisition setup as shown in Figure 1, the light source is located at the optical center. The surface is represented by [9,22]: $\mathcal{S}=\{S(\mathbf{x}) / \mathbf{x} \in \Omega\}$, where $S(\mathbf{x})=\frac{f u(\mathbf{x})}{\sqrt{|\mathbf{x}|^{2}+f^{2}}}(\mathbf{x},-f)$, with $f$ is the camera's focal length.

\section{Method}

\section{Data Preprocessing}

The triangular meshes of the training ensemble are obtained from a high resolution computer tomography scan of human invitro teeth where we use the ExpectationMaximization (EM) algorithm for segmentation. 3D surfaces for each tooth type are rigidly aligned using an ICP-based rigid registration algorithm [85] using the Hausdorff distance between corresponding points.

\section{Shape Model Construction}

In this chapter, shape reconstruction, using 2D-PCA, is using the height map ${ }^{11}$ in order to extract the most significant information of training images. Unlike the conventional PCA, 2D-PCA as the name implies will have matrix information rather than vector information which means that there is no need to get image pre-transformed into a vector,

\footnotetext{
${ }^{11}$ a height map is a raster image used to store values, such as surface elevation data (The depth in the proposed case)
} 
let more coefficients will be needed to represent the image [115-117]. This needs incorporation of the conventional PCA in a further step to reduce the dimensionality of principle component matrix of 2D-PCA.

Now, let the training set consist of $M$ training height maps $\left\{U_{1}, \ldots, U_{M}\right\}$ with size $n \times m$. All images are pre-aligned. As in [115-117], the mean of the training shapes, $\bar{U}$, is obtained as the average of these $M$ height maps. To extract the shape variabilities, $\bar{U}$ is subtracted from each of the training height maps. The obtained mean-offset functions $\left(\widehat{U}_{i}=U_{i}-\bar{U}, i=1, \cdots, M\right)$ can be represented as $\left\{\widehat{U}_{1}, \ldots, \widehat{U}_{M}\right\}$. These new functions are used to measure the variabilities of the training images. $M$ training teeth (for each type) images with $100 \times 100$ pixels are used in the experiment. According to [115-117], the $M$ mean-offset height maps are used to construct the covariance matrix $\mathbf{G}$, as following:

$$
\mathbf{G}=\frac{1}{M} \sum_{i=1}^{M} \widehat{U}_{i}^{T} \widehat{U}_{i}
$$

The goal of 2D-PCA is to find the optimal $K$ eigenvectors of $\mathbf{G}$ corresponding to the largest $K$ eigenvalues. The value of $K$ helps to capture the necessary shape variation with minimum information. Experimentally, the minimum suitable value is found to be $K=10$ that give enough variations. After choosing the eigenvectors corresponding to the 10 largest eigenvalues ( $\left.\mathbf{B}=\mathbf{b}_{1}, \mathbf{b}_{2}, \ldots, \mathbf{b}_{10}\right)$, the principle component matrix $\mathbf{Y}_{i}(m=100 \times K=10)$ are obtained for each height map of the training set $(i=1,2, \ldots, M)$, where $\mathbf{Y}_{i}=U_{i} \mathbf{B}^{T}$. For more dimensional reduction, the conventional PCA is applied on the principle components $\left\{\overrightarrow{\mathbf{Y}}_{1}, \ldots, \overrightarrow{\mathbf{Y}}_{\mathbf{M}}\right\}$. It should be noted that, $\overrightarrow{\mathbf{Y}}$ is the vector representation of $\mathbf{Y}$. The reconstructed components (after retransforming to matrix representation) will be:

$$
\widetilde{\mathbf{Y}}_{\{l, \mathbf{h}\}}=\mathbf{D} \mathbf{e}_{\{l, \mathbf{h}\}}
$$

Where $\mathbf{D}$ is the matrix which contains $L$ eigenvectors corresponding to $L$ largest eigenvalues 
$\lambda_{l},(l=1,2, \ldots, L)$, and $e_{\{l, h\}}$ is the set of model parameters which can be described as:

$$
\mathbf{e}_{\{l, h\}}=h \sqrt{\lambda_{l}}
$$

where $l=\{1, \ldots, L\}, h=\{-\mu, \ldots \mu$,$\} , and \mu$ is a constant which can be chosen arbitrarily. The new principle components of training height maps are represented as $\left\{\widetilde{\mathbf{Y}}_{1}, \ldots, \widetilde{\mathbf{Y}}_{\mathbf{N}}\right\}$ instead of $\left\{\mathbf{Y}_{1}, \ldots, \mathbf{Y}_{M}\right\}$ where $N$ is a constant which can be chosen arbitrarily.

Given the set $\left\{\widetilde{\mathbf{Y}}_{\mathbf{1}}, \ldots, \widetilde{\mathbf{Y}}_{\mathbf{N}}\right\}$, the new projected training height maps are obtained as:

$$
\widetilde{\mathbf{U}}_{n}=\widetilde{\mathbf{Y}}_{n} \mathbf{B}^{T}, \quad n=1,2, \ldots . ., N
$$

The shape model is required to capture the variations in the training set. This model is considered to be a weighted sum of the projected height maps (49) as:

$$
u(\mathbf{x})=\overline{\mathbf{U}}+\sum_{n=1}^{N} w_{n} \widetilde{\mathbf{U}}_{n},
$$

where $\overline{\mathbf{U}}$ is the mean height map (mean tooth shape), $\widetilde{\mathbf{U}}_{n}$ is the $n^{\text {th }}$ orthogonal mode of variation in the shape (also called eigenteeth), and $\mathbf{w}=\left\{w_{n}\right\}$ is called the vector of eigen coefficients or the shape vector. Only $N$ principal components are considered in the sum, where $N$ should be chosen large enough to be able to capture the prominent shape variations present in the human teeth.

The function given in Equation 50 is used as explicit representation of teeth shape. Therefore, by varying $\mathbf{w}, u(\mathbf{x})$ is varied.

\section{Integration Tooth Shape Priors into SFS-framework}

In this phase, the prior shape model is embedded in SFS framework to guide the 
solution. The Oren-Nayar-Wolff diffuse reflection model with a near light source and perspective camera is considered.

As explained before in VI.B, alignment is needed before the construction step between the input brightness image to be reconstructed and the constructed shape model. A 2D rigid image registration technique with maximization of mutual information [113] is conducted to achieve the alignment. The Oren-Nayar-Wolff model is applied for reflectance with a camera that obeys perspective projection and a light source is located at the cameras optical center. Intensity of the light is attenuated with squared distance. The idea here is to solve for the height map $u(\mathbf{x})$ that minimizes the energy functional

$$
\varepsilon=\int_{\Omega}\left(I(\mathbf{x})-R(u(\mathbf{x}))^{2} d \mathbf{x},\right.
$$

where

$$
R(u(\mathbf{x}))=\frac{A\left(1-F(\theta, \zeta)^{2} \cos \theta+B \sin ^{2} \theta\right.}{r^{2}}
$$

From the definitions of the perspective camera, $\cos \theta$ can be written as:

$$
\cos \theta=\frac{f}{\sqrt{x^{2}+y^{2}+f^{2}}} u(\mathbf{x})
$$

A great advantage of the above optimization problem (51) is that the solution search space is shrunk into a finite number $(N)$ of weights. The goal here is to find the solution:

$$
\hat{w}_{n}=\arg \min _{w_{n}} \varepsilon, n=1, \cdots, N,
$$

Gradient descent optimization is used for computing $w_{n}$ as follows:

$$
\frac{\partial w_{n}}{\partial t}=-\eta \frac{\partial \varepsilon}{\partial w_{n}},
$$


where $\eta$ is real positive learning constant. The gradient will be as follows:

$$
\frac{\partial \varepsilon}{\partial w_{n}}=\int_{\Omega} 2[I(\mathbf{x})-R(u(\mathbf{x}))]\left(-\frac{\partial R(u(\mathbf{x}))}{\partial w_{n}}\right) d \mathbf{x} .
$$

\section{E. Experimental Results}

\section{Reconstruction of the Single Tooth}

To evaluate the performance of the proposed 3D reconstruction method, the proposed approach is applied on real human teeth. Premolar model, Mandibular molar, Mandibular third molar, Maxillary molar, and maxillary deciduous models are constructed from $30,30,100,40$, and 30 teeth respectively. The shape priors are trained using out-of-training samples with instances using the CT-scan of the respective teeth. The accuracy of the tooth reconstruction is assessed by comparing it to the $3 \mathrm{D}$ ground-truth surface as obtained from CT scan. The proposed algorithm (AAA) is evaluated with two algorithms. The first algorithm (DD) [21] is more recent. It is a SFS algorithm that reconstructs 3D shape of the human teeth based on the shape priors that are built using conventional PCA. The other algorithm (B) [6] is a conventional SFS approach based on the work of Ahmed and et al. in [12].

Table 5 summarizes the key differences between the proposed algorithm (AAA) and the others algorithms (DD and B).

Figure 38 illustrated samples results from the three approaches under evaluation. Figure 38(a) demonstrates the 2D input images for different teeth models (e.g. mandibular molar, mandibular third molar, maxillary molar, and maxillary deciduous). Figure 38(b) shows the corresponding ground-truth (GT) as obtained from CT scans. Figure 38(c) shows the results of the proposed method AAA. Figure 38(d) shows the 3D reconstruction of 
TABLE 5: Summary of approaches under experimental comparison. All algorithm are a perspective camera projection and the light source at the camera optical center.

\begin{tabular}{|c|c|c|c|}
\hline & Reflectance & Priors & Reference \\
\hline AAA-new & Oren-Nayar-Wolff & 2D-PCA & Proposed \\
DD & Oren-Nayar & PCA & {$[21]$} \\
B & Oren-Nayar & no & {$[6]$} \\
\hline
\end{tabular}

TABLE 6: Average tooth surface reconstruction accuracy $(R M S)$ in $m m$.

\begin{tabular}{|c|c|c|c|}
\hline Tooth Type & Proposed (AAA) & algorithm (DD) [21] & SFS (B) [6] \\
\hline \hline Premolar & 0.2872 & 0.6502 & 1.3739 \\
\hline Mandibular molar & 0.3017 & 0.6825 & 1.1098 \\
\hline Mandibular third molar & 0.2058 & 0.5625 & 1.0702 \\
\hline Maxillary molar & 0.3288 & 0.6646 & 1.2738 \\
\hline Maxillary deciduous & 0.2591 & 0.5711 & 1.4317 \\
\hline
\end{tabular}

the human teeth using algorithm (DD) while the 3D reconstruction using the traditional well-known SFS in Figure 38 (e). Clearly, better construction is provided by the proposed method. The root-mean-square $(R M S)$ error is measured between the reconstruction surface and the GT after performing the $3 \mathrm{D}$ rigid registration.

The average teeth reconstruction accuracy (RMS) in $m m$ for algorithms AAA, DD and $\mathrm{B}$ is compared for various tooth types in Table 6. It is clear that the proposed algorithm (AAA) outperforms the other algorithms. It is worth-mentioning that throughout the experimentations the proposed method is faster than algorithm (DD). The CPU timing is computed on a PC with Core i7 CPU@ 2.2GHz processor and 4GBRAM. The average time for the proposed method is 40 seconds. while the other algorithm (DD) is 80 seconds. 

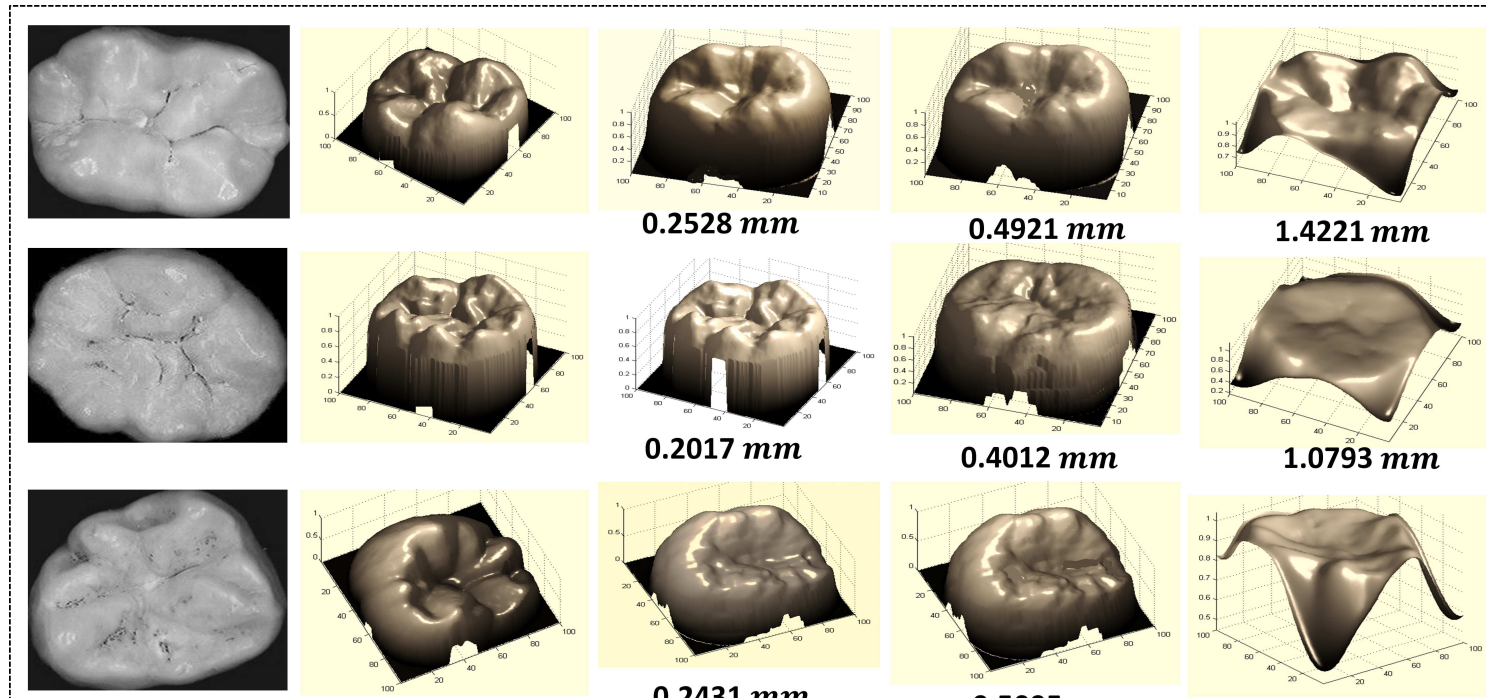

$0.2017 \mathrm{~mm}$
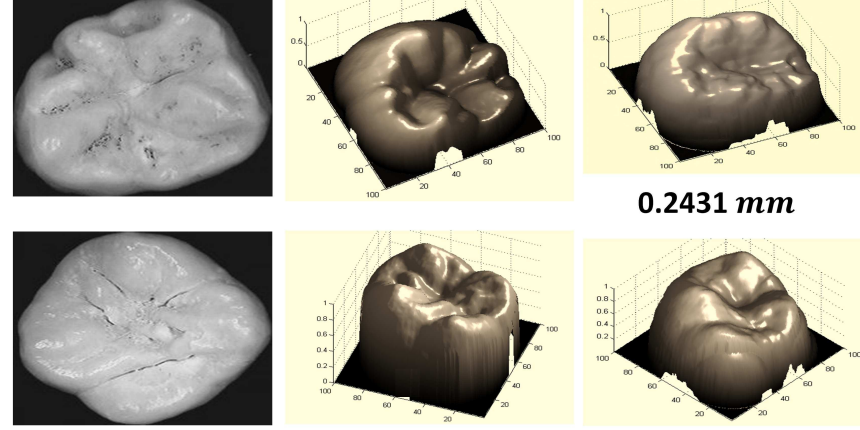

$0.2431 \mathrm{~mm}$
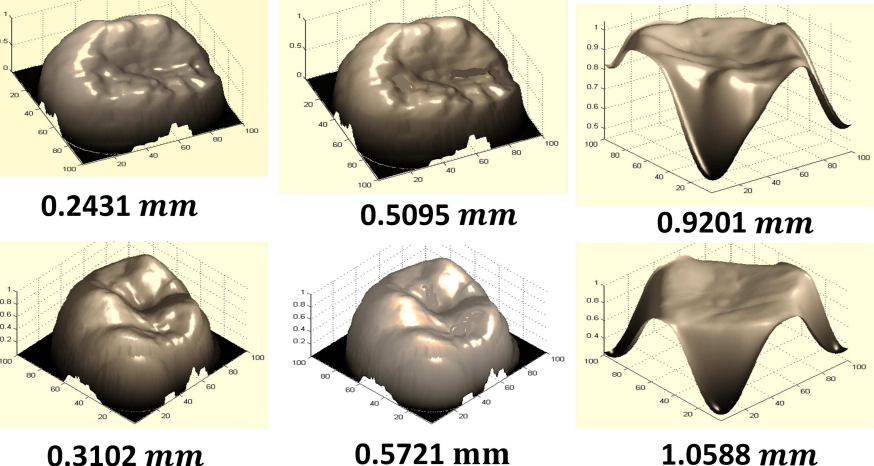

$0.3102 \mathrm{~mm}$

(d)

$1.0588 \mathrm{~mm}$

(a)

(b)

(c)

(e)

FIGURE 37: Tooth reconstruction from three different algorithms. (a) 2D-image captured by the intraoral camera.(b) GT occlusal surface generated from a CT scan of the tooth. (c) 3D Reconstruction of the human teeth using the proposed method AAA. (d) Reconstruction using Algorithm DD. (e) Reconstruction using Algorithm B(well-known SFS method). Beneath each reconstruction is the root-mean-square $(R M S)$ error. 
2. Reconstruction of the Whole Jaw

The triangular meshes of the training ensemble are obtained from a high resolution computer tomography $\left(\mathrm{CT}^{12}\right)$ scan of human jaw molds where the ExpectationMaximization (EM) algorithm for segmentation is used. Dense correspondence between jaw samples is obtained using the 3D thin-plate spline where the alignment procedure is guided by the sparse set of anatomical jaw landmarks. 3D thin-plate spline is also used to provide a warping function between image pixels (assumed to be on the xy-plane in the 3D space) and surface points using image landmarks and surface landmarks as control points. Orthographic projection is applied to re-represent the triangular meshes in terms of Monge patches which provides a bijective mapping between surface points and image coordinates.

In order to evaluate the performance of the proposed approach, several experiments are carried out on real human jaw, other than the ones used for constructing the shape prior model. Upper jaw models are constructed from 52 upper jaw molds belonging to 33 males and 19 females with on average age of 20 years old. Whereas lower jaw models are constructed from 58 lower jaw molds belonging to 33 males and 25 females with on average age of 19 years old. There are two samples per subject, one pre-repair jaw and another post-repair jaw, referring to the jaw status before and after applying an orthodontic teeth alignment process, respectively. The shape priors are trained using out-of-training samples with pre- and post-repair instances using the CT-scan of the respective molds(lower and upper jaws). The accuracy of the jaw reconstruction is assessed by comparing it to the 3D ground-truth surface as obtained from CT scan.

The proposed algorithm is compared $\left(A_{w}\right)$ with two algorithms. The first algorithm $\left(B_{w}\right)$ is Lambertian statistical SFS(SSFS) and recent for tooth reconstruction [118]. The other algorithm $\left(C_{w}\right)[6]$ is a conventional SFS approach based on the work of Ahmed and et al. [12]. In [12], their work is based on viscosity solutions for Hamilton-Jacobi type

\footnotetext{
${ }^{12}$ A Cone-beam CT (KODAK 9000 3D Extraoral) scanner is used.
} 
formulation of the image irradiance equation. This algorithm was developed in [9] for the Lambertian case assuming a perspective camera projection and a light source at the camera optical center. Later [12] was extended to the Oren-Nayar reflectance model. 

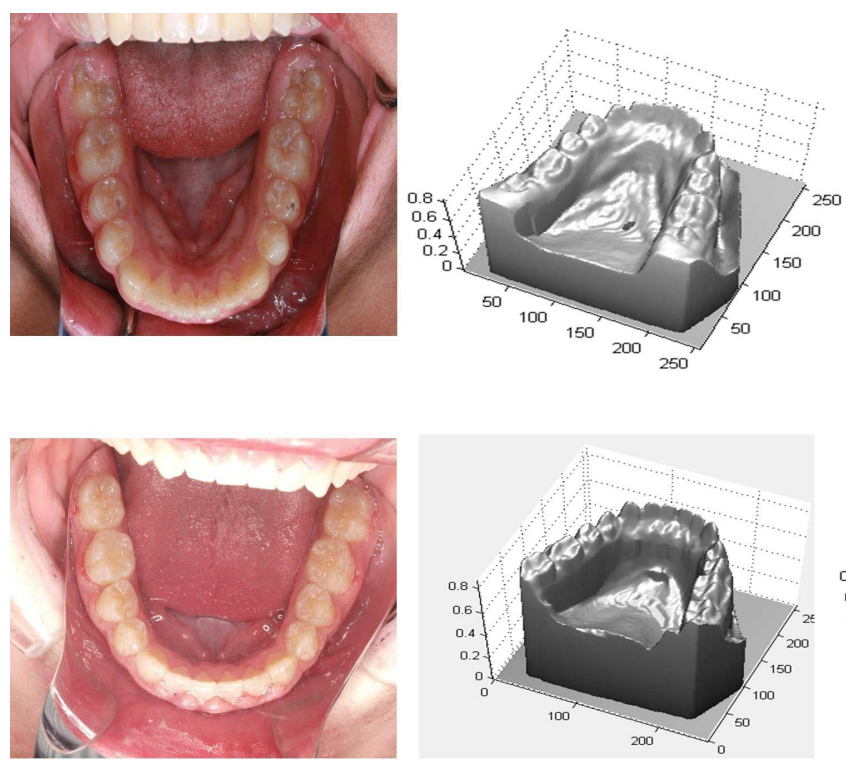

(a)

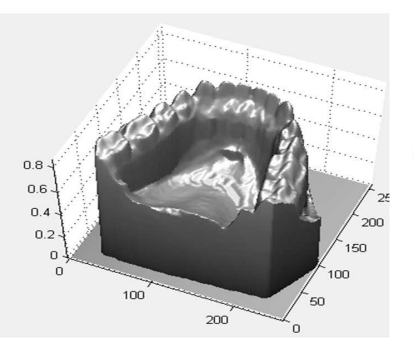

(b)
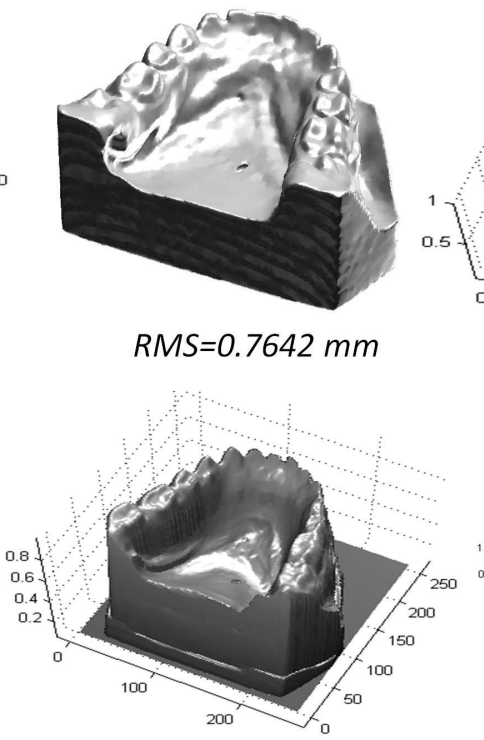

$R M S=0.6804 \mathrm{~mm}$

(c)
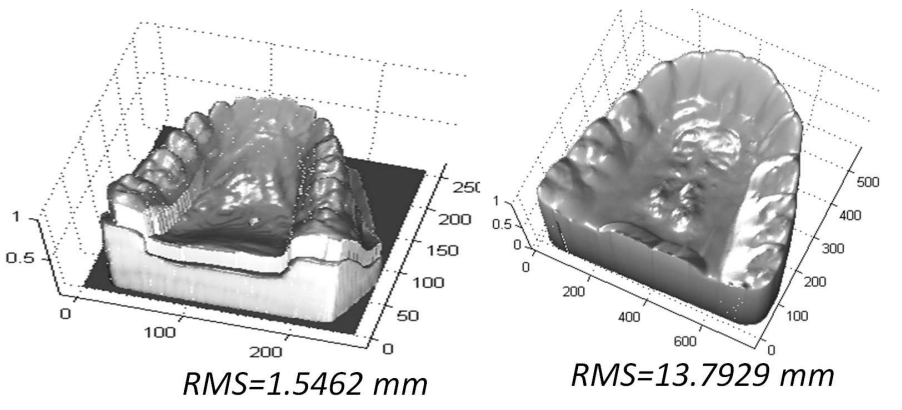

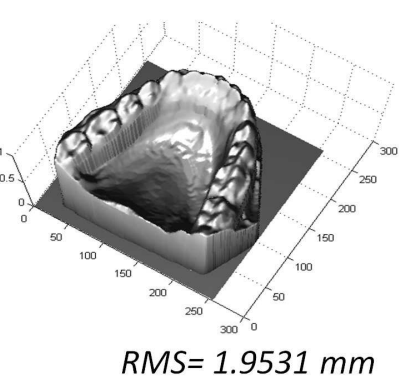

(d)

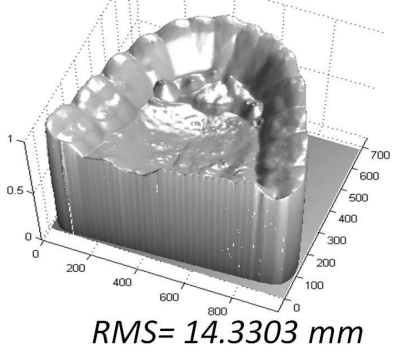

(e)

FIGURE 38: Sample reconstruction result of a lower jaw (Post-repair)from three different algorithms. (a) Image acquired by the CCD camera.(b) Ground-truth generated from a CT scan of the jaw. (c) Reconstruction using the proposed Algorithm $A_{w}$ (with shape priors). (d) Reconstruction using Algorithm $B_{w}$. (e) Reconstruction using method (Algorithm $C_{w}$ ). Beneath each reconstruction is the root-mean-square (RMS) error when compared to the ground-truth surface from CT. 
TABLE 7: Summary of approaches under experimental comparison.

\begin{tabular}{|c|c|c|c|}
\hline & Reflectance & Priors & Reference \\
\hline$A_{w}$ & Oren-Nayar-Wolff & 2D-PCA & Proposed \\
$B_{w}$ & Lambertian & PCA & {$[118]$} \\
$C_{w}$ & Oren-Nayar & No & {$[6]$} \\
\hline
\end{tabular}

Table 7 summarizes the key differences between the proposed algorithm $\left(A_{w}\right)$ and the others algorithms $\left(B_{w}\right.$ and $\left.C_{w}\right)$.

The isosurface of the jaw scan is a metric reference to rigidly align the reconstructed $3 \mathrm{D}$ point from SFS to share the same metric coordinate frame. For alignment, the first and second moments are normalized [96] without affecting the scale. This initializes an ICP-based rigid registration algorithm [85]. Point correspondence between CT and the reconstructed surface is obtained based on Hausdorff distance [97]. The SFS algorithms are compared in accordance with an error estimator based on the root mean square $(R M S)$ error between the 3D points from the CT scan and the corresponding reconstructed surface points.

Sample results from the approaches under evaluation are demonstrated in Figure 38. Figure 38(a) shows the 2D input images for lower jaws, while Figure 38(b) shows the corresponding ground-truth tooth surface as obtained from CT scans. The output of the proposed algorithm $\left(A_{w}\right)$ is shown in Figure 38(c). reconstruction by Algorithms $\left(B_{w}\right.$ and $C_{w}$ ) are shown in Figure 38(d), and Figure 38(e) respectively. Clearly, reconstruction using the formulations with algorithm $\left(A_{w}\right)$ (Figure 38(c)) have shown the most success in that regard. Beneath each reconstruction is the root-mean-square $(R M S)$ error between the reconstruction and the ground-truth after performing 3D rigid registration. Consistently, the proposed algorithm $\left(A_{w}\right)$ provides the smallest RMS errors.

The average jaws reconstruction accuracy $(R M S)$ in $m m$ for algorithms $A_{w}, B_{w}$ and 
TABLE 8: Average whole jaw surface reconstruction accuracy $(R M S)$ in $m m$.

\begin{tabular}{|c|c|c|c|}
\hline Jaw Type & Proposed $\left(A_{w}\right)$ & Lambertian SSFS $\left(B_{w}\right)$ & $\operatorname{SFS}\left(C_{w}\right)$ \\
\hline Upper, Pre-repair & 0.6805 & 2.0899 & 15.2995 \\
\hline Upper, Post-repair & 0.6015 & 2.0233 & 16.3098 \\
\hline Lower, Pre-repair & 0.7281 & 3.1191 & 12.1241 \\
\hline Lower, Post-repair & 0.7759 & 2.5711 & 13.4959 \\
\hline
\end{tabular}

$C_{w}$ is compared for various jaw types in Table 8. It is clear that the proposed algorithm $\left(A_{w}\right)$ outperforms the other algorithms.

To further demonstrate the gain out of the shape priors, another experiment is performed on real human jaws with fillings that cover significant parts of the teeth, see Figure 39(a). Ground-truth generated from a CT scan of the jaw shown in Figure 39(b). Figure 39(c) shows the corresponding surface reconstructions by the proposed algorithm $\left(A_{w}\right)$. Note that the teeth are successfully and completely reconstructed and outperform the work [6] (see Figure 39(d)), in spite of the tooth filling regions with different colors and albedo characteristics in the input images. This notable outcome of the proposed approach becomes more evident when compared to the tooth reconstruction by Algorithm $C_{w}$ in Figure 39(d). The filling regions gave rise to significant valleys and grooves in Algorithm $\left(C_{w}\right)$ results. 

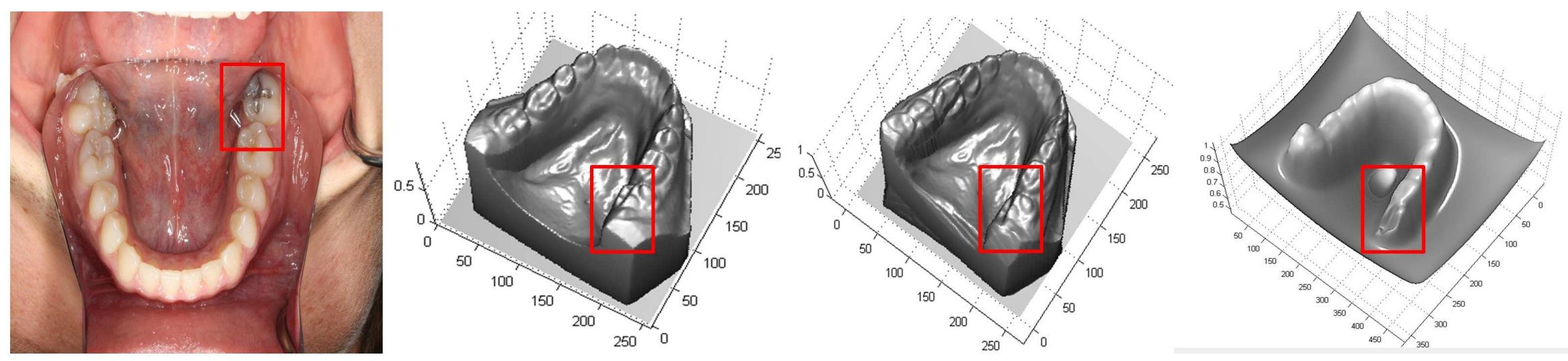

\section{$R M S=0.7325 \mathrm{~mm}$}
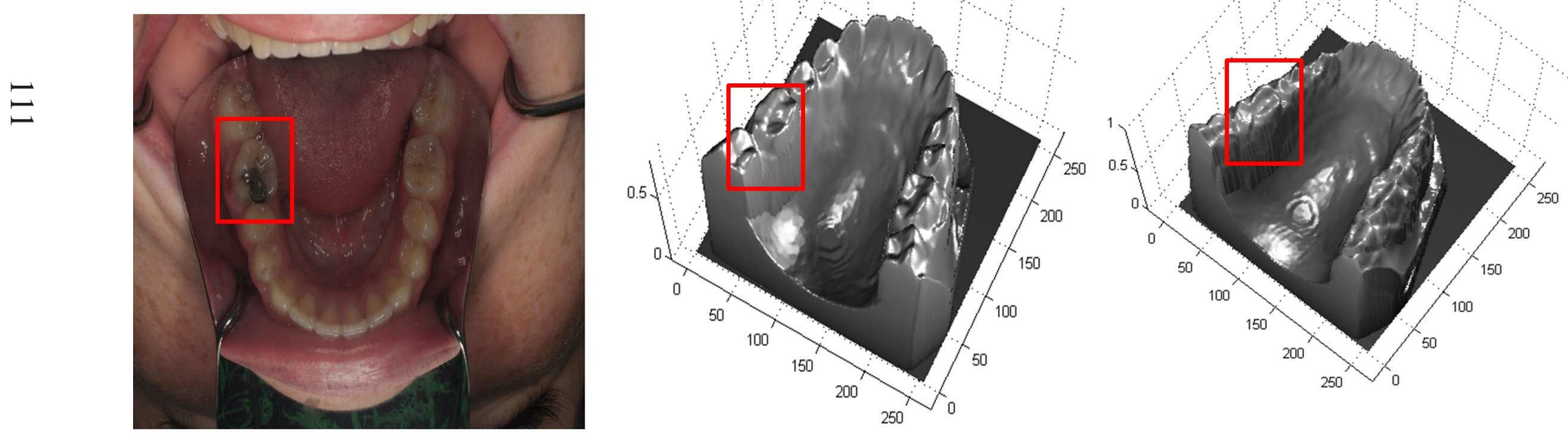

$R M S=0.7905 \mathrm{~mm}$

(a)

(b)

(c)

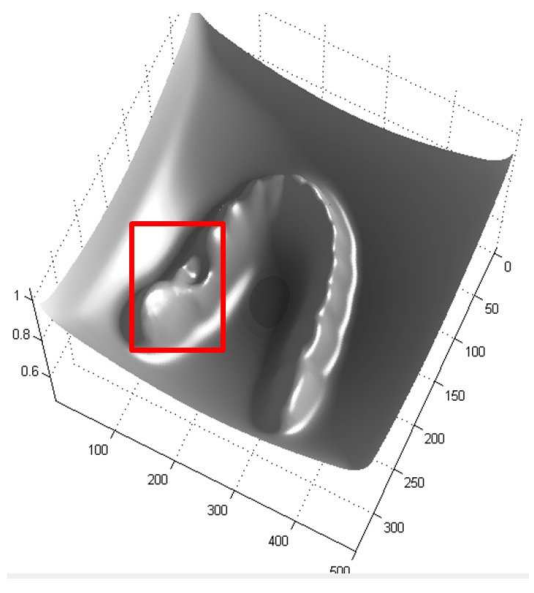

$R M S=15.4871 \mathrm{~mm}$

(d)

FIGURE 39: Experiment on teeth with significant fillings. (a) Input test image. (b) Reconstruction by the proposed algorithm $\left(A_{w}\right)$. (c) Reconstruction by algorithm $\left(C_{w}\right)$ (with no shape priors). 


\section{F. Summary}

A $3 \mathrm{D}$ reconstruction of human tooth models from optical imagery is proposed using shape from shading with 2D-PCA shape priors. The proposed method improves on the 3D reconstruction of the human teeth by incorporating more sophisticated reflectance models. In dental application, improvements are considered significant even if the improvement is a fraction of millimeter. The Oren-Nayar-Wolff reflectance model is used which is a physically deep model for diffuse reflectance from shiny but slightly rough surfaces. Results are preferred to other approaches in the literature since fine tooth details can be reconstructed and the resulting surface is more realistic. Also the execution time is reasonable and practical in the sense of applying the proposed system in clinic. Another major difference in the proposed approach, the output file of the proposed approach, will be readily available as an STL file instead of locked in a proprietary format such as commercial systems have done.

Moreover, the focus has been on developing and validating a holistic approach for image-based 3D reconstruction of the human jaw. The accuracy of 3D reconstruction of the human teeth/jaw is increased using SFS with 2D-PCA shape priors. The proposed approach has successfully reconstructed teeth with challenging conditions, such as scattered specular spots and significant changes in color and albedo characteristics. 


\section{CHAPTER VIII}

\section{THREE-DIMENSIONAL MODELING AND STRESS ANALYSIS IN DENTAL BIOMECHANICS USING FINITE ELEMENT ANALYSIS}

Finite Element Analysis (FEA) or Finite Element Methods (FEM) is a powerful analytic technique for calculating stresses and strains of dental structures. Many Finite Element (FE) studies carried out used approximate 2D models. In this chapter, an accurate three-dimensional CAD model is proposed. 3D stress and displacements of different teeth type are successfully carried out. A newly developed open-source finite element solver, Finite Elements for Biomechanics (FEBio), has been used. The limitations of the experimental and analytical approaches used for stress and displacement analysis are overcome by using FEA tool benefits such as dealing with complex geometry and complex loading conditions. The experiments provide qualitative and quantitative metric results for the five models (Anterior tooth, mandibular third molar, mandibular molar, maxillary third molar and two touched teeth) under different loading conditions.

\section{A. Introduction}

Teeth which are positioned improperly are corrected by orthodontic dentistry. Crooked teeth and teeth which do not fit together properly affects one's health since they are harder to clean and are at continuous risk of early tooth decay and periodontal disease while also causing headaches as well as shoulder and back pain due to extra stress on the chewing muscles.

Bone remodeling and orthodontic tooth movement are initiated by the mechanical 


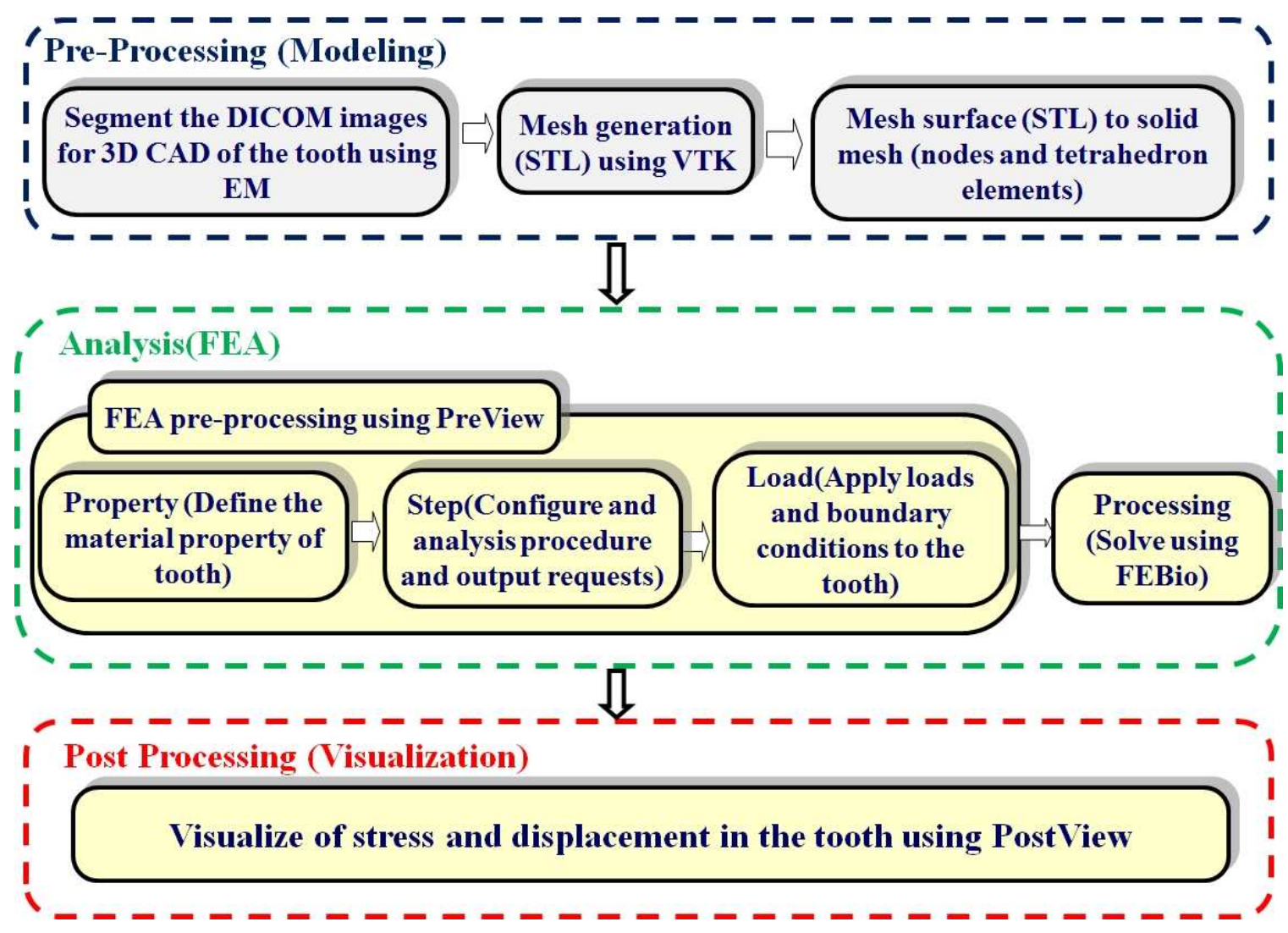

FIGURE 40: Overall approach of the proposed method.

activation of an orthodontic appliance where force systems are produced to displace teeth in a predictable as well as controllable manner. Force systems primarily involve moments and forces where the ratio of moment to force being applied to teeth control the type of tooth movement. After an immediate application of a force, the tooth moves by an elastic deformation of its periodontal ligament (PDL) which is a lining around the root of the tooth. The mechanical stress in the PDL initiates the bone remodeling process which results in orthodontic tooth movement. Such a process is triggered by changes in the stress/strain distribution; it involves bone formation in tension regions and bone resorption (breakage) in stretched regions. In literature, there are several theories that explain the bone remodeling process [119] such as pressure-tension theory, distortion or bending of the alveolar bone and alveolar bone apposition.

Several biomechanical models have been proposed in literature. They are mainly based on the computation of stress and strain distribution in the PDL combined with a 
bone remodeling law [120]. For example, Provatidis [121] and Schepdael [122] proposed an analytical model to describe the stresses and strains in the periodontal ligament of an axisymmetric tooth of paraboloid shape during translation. The PDL was assumed to be linearly elastic and the method was validated using finite element methods. While this analytical model provides easier and lower-computational cost compared to classical FE methods without sacrificing accuracy, this work lacks two main aspects: (1) it did not address the issue of estimating the geometric parameters of the PDL given the 3D root information and (2) it did not address the effect of applying a force system to the whole jaw where the movement of one tooth will exert force on neighboring teeth.

There has been an enormous increase in use of FEA tools (e.g. [123-129]) due to evolution in computational approaches using commercial tools such as Abaqus, ANSYS, SolidWorks, etc. Most of these studies have been simplified work and used 2D analysis techniques. Unfortunately, such assumptions are no longer held in the actual scenario present in the dental structure in terms of geometry. Furthermore, these commercial softwares are very expensive because they require licences.

In this chapter, computational models are investigated they can describe tooth movement process in order to be a useful dental tool in clinical practice as well as research. Different theories provide means for the calculation of the stress and strain levels in the PDL. While in research, this might enable validating a specific remodeling theory; this can also be used in clinical practice to (1) predict tooth movement given orthodontic appliance (force system), (2) simulate tooth movement for orthodontics, and (3) analyze the stress/strain field in the alveolar bone to quantify treatment reaction. In this study, an accurate threedimensional CAD model is proposed. 3D stress and displacement of five different teeth using FEA solver are successfully measured. In particular, FEBio [130] is used which is a newly developed open-source finite element solver. To the best of knowledge this has not been used before in orthodontic. FEBio has been developed specifically for biomechanical applications [130]. Nonetheless, the effect of applying a force system to the two teeth is 
addressed where the movement of one tooth will exert force on neighboring teeth.

\section{B. Orthodontic Tooth Movement}

Teeth which are positioned improperly are corrected by orthodontic dentistry. Crooked teeth and teeth which do not fit together properly affects ones health since they are harder to clean and are at continuous risk of early tooth decay and periodontal disease while causing headaches shoulder and back pain due to extra stress on the chewing muscles.

Bone remodeling and orthodontic tooth movement are initiated by the mechanical activation of an orthodontic appliance where force systems are produced to displace teeth in a predictable as well as controllable manner. Force systems primarily involves moments and forces where the ratio of moment to force being applied teeth control the type of tooth movement. After an immediate application of a force, the tooth moves by an elastic deformation of its periodontal ligament (PDL) which is a lining around the root of the tooth. The mechanical stress in the PDL initiates the bone remodeling process which results in orthodontic tooth movement. Such process is triggered by changes in the stress/strain distribution; it involves bone formation in tension regions and bone resorption (breakage) in stretched regions. In literature, there are several theories explain the bone remodeling process [119] such as pressure-tension theory, distortion or bending of the alveolar bone and alveolar bone apposition.

Although there exist some typical and known orthodontic treatment plans, to-date, such treatment is primarily dependent on the experience of the orthodontist. Further, the treatment reaction is different from one patient to another causing lengthening the treatment time due to plan adjustment while increasing the cost to the patient. Time, cost and patient inconvenience are the main motivations behind having a simulation tool for orthodontic tooth movement where the orthodontist can virtually apply different treatment plans and simulate bone reactions versus time. Such a tool requires: (1) a 3D representation of the 
patients visible jaw, hence our 3D reconstructions come into play, (2) augmenting the visible jaw with 3D root information and (3) modeling tooth movement w.r.t. different moment to force ratios, i.e. different movement types (e.g. rotation, translation and tipping).

On the tooth movement side, we are seeking to investigate whether computational models can describe tooth movement process in order to be a useful dental tool in clinical practice as well as research. Different theories provide means for the calculation of the stress and strain levels in the PDL. While in research, this might enable validating a specific remodeling theory, this can also be used in clinical practice to (1) predict tooth movement given orthodontic appliance (force system), (2) simulate tooth movement for orthodontics, and (3) analyze the stress/strain field in the alveolar bone to quantify treatment reaction. Nonetheless, the outcome of these theories is dependent on three main factors: (1) Geometry and morphology where tooth and alveolar bone are typically considered as rigid bodies while differences according to micromorphology have not been yet addressed [119]. (2) Material properties where PDL is non-linear visco-elastic material while most works consider it homogeneous, linear-elastic and isotropic. (3) Boundary conditions where the type of tooth movement is controlled by the moment to force ratio.

In literature, different approaches for modeling orthodontic tooth movement involve designing either a biomechanical or a mechanobiological model. While the former focus on the mechanics of the process while taking into account that bone and tissues are constantly adapting living tissues. The later models the biological processes involved in bone formation and resorption including cell and growth factor concentrations [131]. Nonetheless, such types of models are more complex and computationally expensive for clinical use when compared to biomechanical ones due the complex nature of cells and growth factors [131]. Yet, they are suited as research tools.

Several biomechanical models have been proposed in literature. They are mainly based on the computation of stress and strain distribution in the PDL combined with a bone remodeling law [120]. For example, Provatidis [121] proposed an analytical model 
to describe the stresses and strains in the periodontal ligament of an axisymmetric tooth of paraboloid shape, during translation. The PDL was assumed to be linearly elastic and the method was validated using finite element (FE) methods. Gei et al. [132] proposed an interface model of the periodontal ligament. Their key idea was to model the PDL as a non-linear interface in a FE model of the root and the alveolar bone. That way, the nonlinearity of the PDL is captured without defining it explicitly as a third material in the FE calculations. The drawback of this approach is that, although results are obtained for the stresses and strains inside the alveolar bone, the stresses and strains inside the PDL are not calculated. Zhurov et al. [133] developed a constitutive model of the PDL, describing it as a compressible transversely isotropic visco-hyperelastic tissue. They took into account the fact that the PDL is a composite material, composed of collagen fibers that can only bear tensile stresses and a viscous matrix component that has resistance to tension and compression. The advantage of such a model is its completeness, but to obtain accurate results, it is essential that all the material parameters are identified correctly, which is not yet the case. Kojima et al. [134-136] assumed that amount of bone remodeling is in proportion to the mean stress being calculated in the PDL. While they were able to simulate tooth movement, the validation of their model in clinical practice is still in question.

Clinical practice would benefit from a low-computational model while being easyto-use for orthodontists. Recently, Schepdael et al. [137] proposed an analytical approach to design a biomechanical model for tooth movement where they avoided the use of classical FE methods to decrease computational time and increase user friendliness. Their approach did not require setting up a complicated 3D model of the tooth. Their model involves two main stages: (1) analytical determination of stress patterns in the PDL during orthodontic tooth movement and (2) simulating bone remodeling process as a viscous process where tooth movement of single- and multi-rooted teeth can be predicted. They validated their model against FE models showing minimal average error with lower computational complexity. While handling multi-rooted teeth, they improved on Provatidis [121] by predicting all types of tooth movements rather than only root translation and better modeling 
the shape of the root using an elliptic paraboloid.

While this analytical model provides easier and lower-computational cost compared to classical FE methods without sacrificing accuracy, this work lacks two main aspects: (1) it did not address the issue of estimating the geometric parameters of the PDL given the 3D root information and (2) it did not address the effect of applying a force system to the whole jaw where the movement of one tooth will exert force on neighboring teeth.

\section{The Finite Element Methods}

The FEM were first invented by structural engineers, who based themselves on accurately physical basis. Later, mathematicians discovered that FEM methods could be classified as a subset of the Galerkin Methods for the solution of PDEs. By this way the method gained a mathematical foundation which extended its use to many engineering problems. None the less this difference in the engineering and mathematics points of view resulted in two different interpretations which also affects the way the method is used in practice applications.

- Physical Interpretation:

The continous physical model is divided into finite pieces called elements and laws of nature are applied on the generic element. The results are then assembled to represent the continuum.

- Mathematical Interpretation:

The differetional equation reppresenting the system is converted into a variational form and solved by the linear combination of a finite set of trial functions. 


\section{FEM Notation}

FEM treat the continuous problem domain as a collection of individual finite elements. The problem parameters are defined on each of the nodes of a typical element. The key definitions of the FEM notation have been described as the follows:

- Dimensionality: The elements can be defined depending on the problem context. Dimensionality expresses whether the element has 1,2 or 3 space dimensions.

- Nodal Points: Every element is described by its nodal points. The nodal points are chosen to be the corners of the element. In the case of non linear geometries nodal points are defined on the edges.

- Geometry: The geometry is used to describe the domain on which finite element discretization needs to be applied as shown in Figure 41.

- Nodal Forces: A set of nodal forces.

2. Physical Problems, Mathematical Models and Finite Element Solution

The finite element analysis method is used to solve physical problems in engineering analysis and design. The flowchart shown in Figure 42 summarizes the process of finite element analysis. The physical problem involves an structure component to certain loads. To convert the physical model to the mathematical model requires certain assumptions that together lead differential equations governing the mathematical model. This model is solved by using finite element analysis. Since the finite element solution approach is a numerical procedure, it is indispensable to rate the solution accuracy. If the estimated accuracy is not met the actual accuracy, the numerical (i.e., finite element) solution has to be repeated with adjusted the solution parameters (such as finer meshes) until a suitable accuracy is reached. 

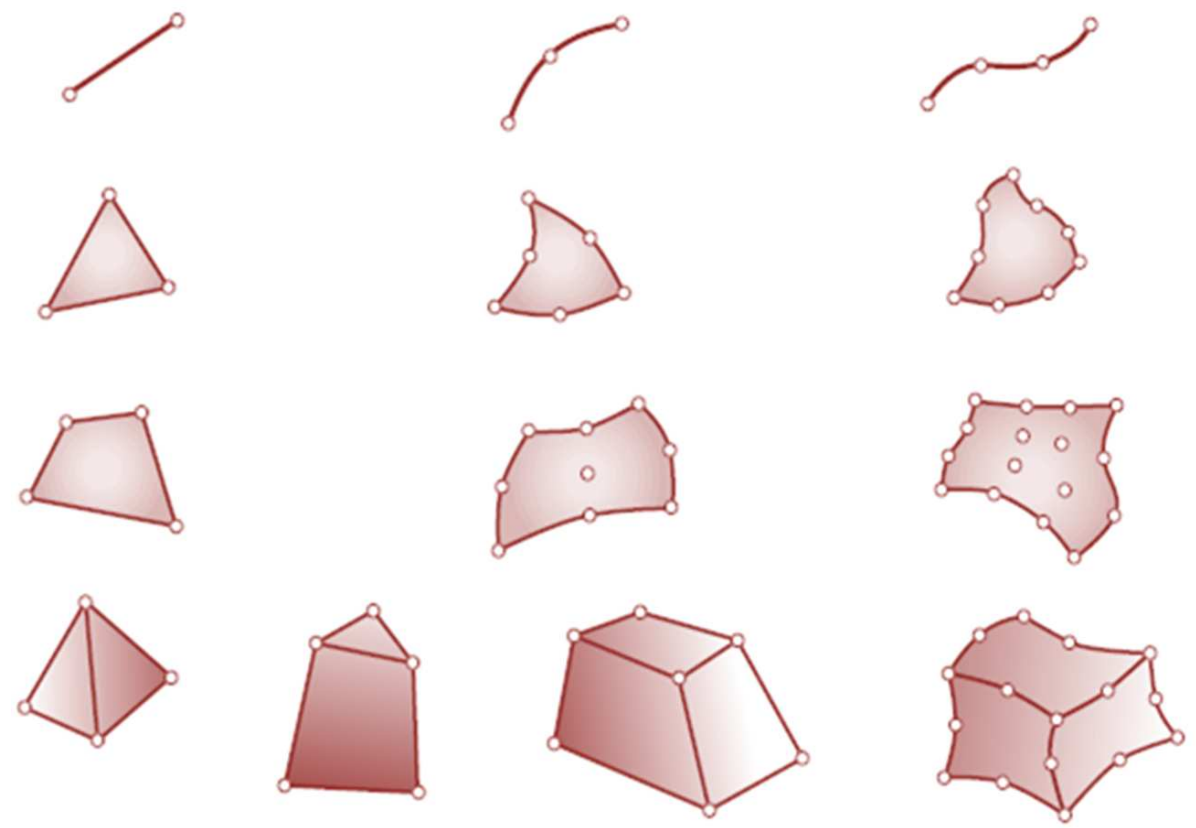

FIGURE 41: Finite element geometries: First row: 1D. Second row: 2D. Third row: 2D. Fourth row: 3D.

\section{Equations for Three-Dimensional Solids}

\section{Stress and Strain}

Figure 43 shows a continuous three-dimensional (3D) elastic solid tooth with a volume $V$ and a surface $S$. The surface of the solid tooth is divided into two types of surfaces: 1) a surface on which the external forces are prescribed is denoted $S_{F}, 2$ ) a surface on which the displacements are prescribed is denoted $S_{d}$. The solid can also be loaded by body force $f_{b}$ and surface force $f_{s}$ in any distributed fashion in the volume of the solid [138].

Figure 44 shows at any point in the solid, the components of stress are indicated on the surface of an 'infinitely' small cubic volume. On each surface, there will be the normal stress component, and two components of shearing stress. The first subscript letter 


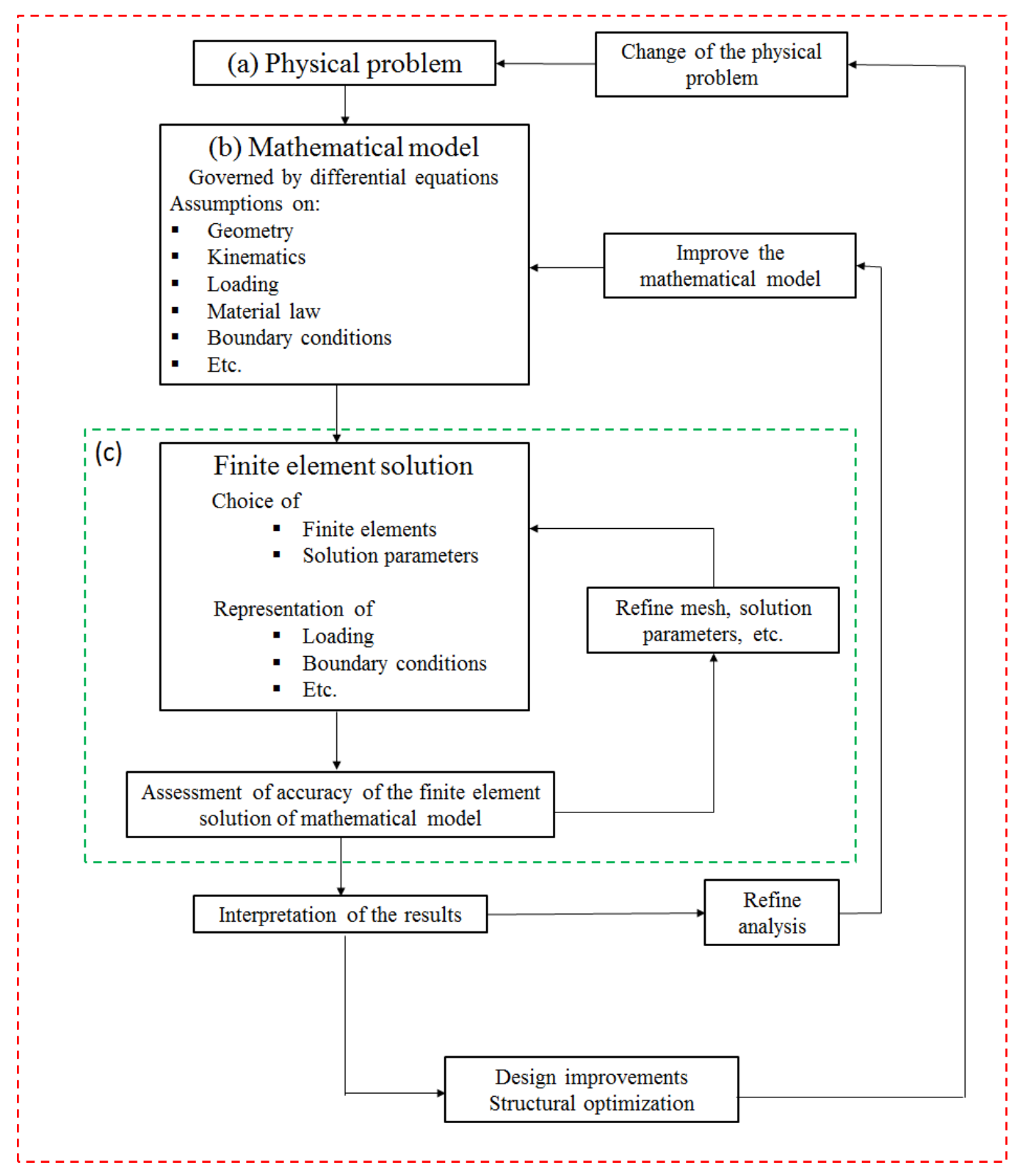

FIGURE 42: The flowchart of the process of finite element analysis: (a) The physical problem. (b) Finite element solution of mathematical model. (c) postprocessing step: interpretation the results, design improvements and structural optimization. 


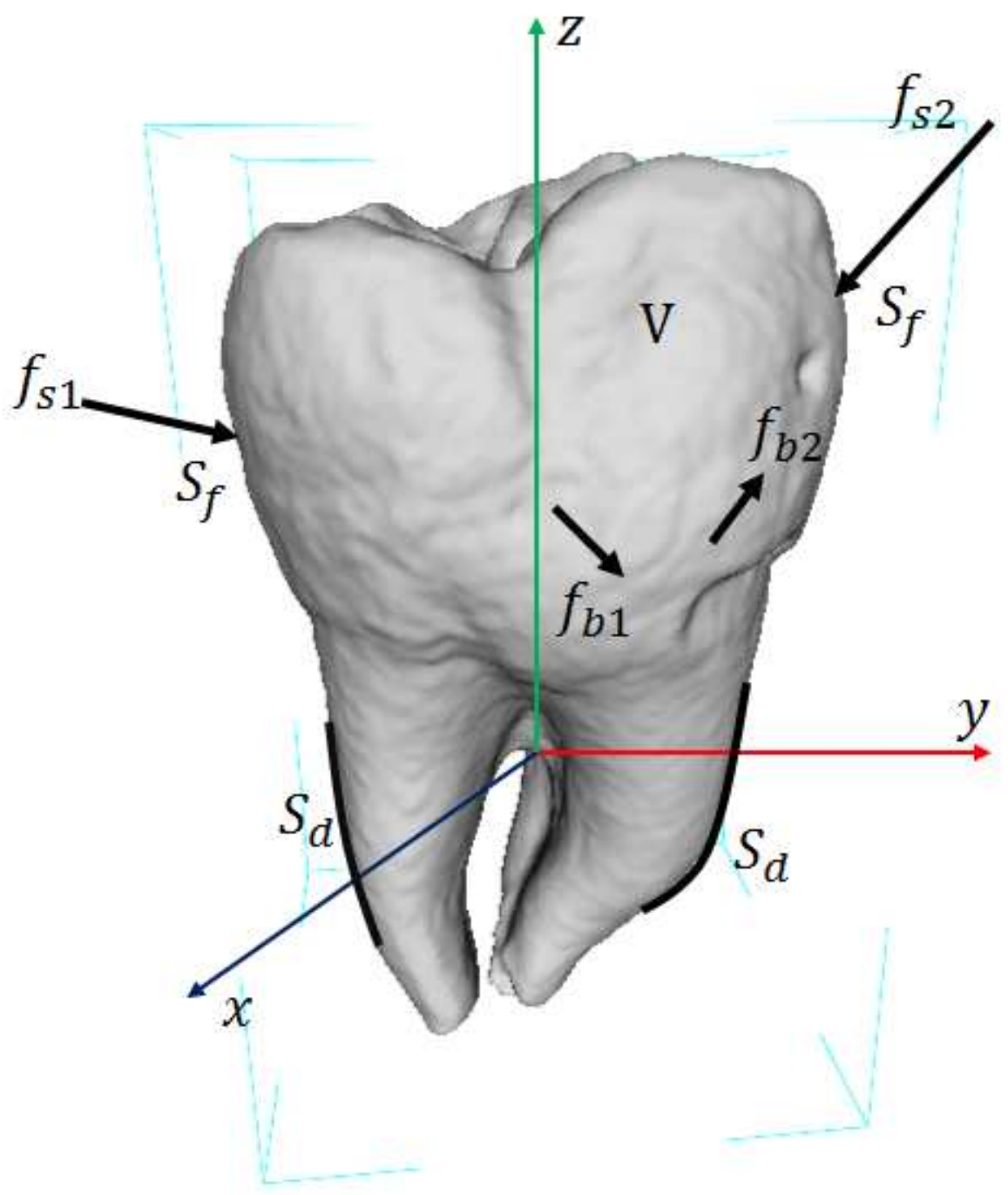

FIGURE 43: Solid tooth subjected to forces applied within the solid (body force) and on the surface of the solid tooth (surface force). 
represents the surface on which the stress is acting, and the second letter represents the direction of the stress. The directions of the stresses illustrated in the Figure 44 are taken to be the positive directions. By calculating the moments of the forces about the central axes of the cube at the state of equilibrium, it is easy to prove that:

$$
\sigma_{x y}=\sigma_{y x} ; \quad \sigma_{x z}=\sigma_{z x} ; \quad \sigma_{z y}=\sigma_{y z}
$$

Thus, there are six stress components in total at a point in solids. These stresses are often called a stress tensor. They are often written in a vector form as follows:

$$
\sigma^{T}=\left\{\begin{array}{llllll}
\sigma_{x x} & \sigma_{y y} & \sigma_{z z} & \sigma_{y z} & \sigma_{x z} & \sigma_{x y}
\end{array}\right\}
$$

At any point in a solid, there are six train components corresponding to the six stress tensors. The six strain components can also be written in a similar vector form of:

$$
\varepsilon^{T}=\left\{\begin{array}{llllll}
\varepsilon_{x x} & \varepsilon_{y y} & \varepsilon_{z z} & \varepsilon_{y z} & \varepsilon_{x z} & \varepsilon_{x y}
\end{array}\right\}
$$

Strain is define as the change of displacement per unit length, and therefore the components of strain can be obtained from the derivatives of the displacements as follows:

$$
\begin{array}{lll}
\varepsilon_{x x}=\frac{\partial u}{\partial x} ; & \varepsilon_{y y}=\frac{\partial v}{\partial y} ; & \varepsilon_{z z}=\frac{\partial w}{\partial z} \\
\varepsilon_{x y}=\frac{\partial u}{\partial y}+\frac{\partial v}{\partial x} ; & \varepsilon_{x z}=\frac{\partial u}{\partial z}+\frac{\partial w}{\partial x} ; & \varepsilon_{y z}=\frac{\partial v}{\partial z}+\frac{\partial w}{\partial y}
\end{array}
$$

where $u, v$ and $w$ are the displacement components in the $x, y$ and $z$ directions, respectively. The six straindisplacement relationships in Equation 60 can be rewritten in the matrix form as: 


$$
\varepsilon=\mathbf{L} \mathbf{U}
$$

where $\mathbf{U}$ is the displacement vector, and has the form of

$$
\mathbf{U}=\left\{\begin{array}{l}
u \\
v \\
w
\end{array}\right\}
$$

and $\mathbf{L}$ is a matrix of partial differential operators obtained simply by inspection on Equation 60:

$$
\mathbf{L}=\left[\begin{array}{ccc}
\partial / \partial x & 0 & 0 \\
0 & \partial / \partial y & 0 \\
0 & 0 & \partial / \partial z \\
0 & \partial / \partial z & \partial / \partial y \\
\partial / \partial z & 0 & \partial / \partial x \\
\partial / \partial y & \partial / \partial x & 0
\end{array}\right]
$$

2. Constitutive Equation

The relationship between the stress and strain in the material of a solid called constitutive equation. It is often termed Hookes law. The generalised Hookes law for general anisotropic materials can be given in the matrix form as follow:

$$
\sigma=\mathbf{c} \varepsilon
$$


where $\mathbf{c}$ is a matrix of material constants, which are normally obtained through experiments. The constitutive equation can be written explicitly as:

$$
\left\{\begin{array}{c}
\sigma_{x x} \\
\sigma_{y y} \\
\sigma_{z z} \\
\sigma_{y z} \\
\sigma_{x z} \\
\sigma_{x y}
\end{array}\right\}=\left[\begin{array}{cccccc}
c_{11} & c_{12} & c_{13} & c_{14} & c_{15} & c_{16} \\
& c_{22} & c_{23} & c_{24} & c_{25} & c_{26} \\
& & c_{33} & c_{34} & c_{35} & c_{36} \\
& & & c_{44} & c_{45} & c_{46} \\
& & & & c_{55} & c_{56} \\
& & & & & c_{66}
\end{array}\right]\left\{\begin{array}{c}
\varepsilon_{x x} \\
\varepsilon_{y y} \\
\varepsilon_{z z} \\
\varepsilon_{y z} \\
\varepsilon_{x z} \\
\varepsilon_{x y}
\end{array}\right\}
$$

Note that, since $c_{i j}=c_{j i}$, there are altogether 21 independent material constants $c_{i j}$ , which is the case for a fully anisotropic material. For isotropic materials, however, $c$ can be reduced to:

$$
\mathbf{c}=\left[\begin{array}{cccccc}
c_{11} & c_{12} & c_{13} & 0 & 0 & 0 \\
& c_{11} & c_{12} & 0 & 0 & 0 \\
& & c_{11} & 0 & 0 & 0 \\
& & & \left(c_{11}-c_{12}\right) / 2 & 0 & 0 \\
& & & & \left(c_{11}-c_{12}\right) / 2 & 0 \\
& & & & & \left(c_{11}-c_{12}\right) / 2
\end{array}\right]
$$

where,

$$
c_{11}=\frac{E(1-v)}{(1-2 v)(1+v)} ; \quad c_{12}=\frac{E v}{(1-2 v)(1+v)} ; \quad \frac{c_{11}-c_{12}}{2}
$$

in which $E, v$ and $G$ are Youngs modulus, Poissons ratio, and the shear modulus of the material, respectively. There are only two independent constants among these three 


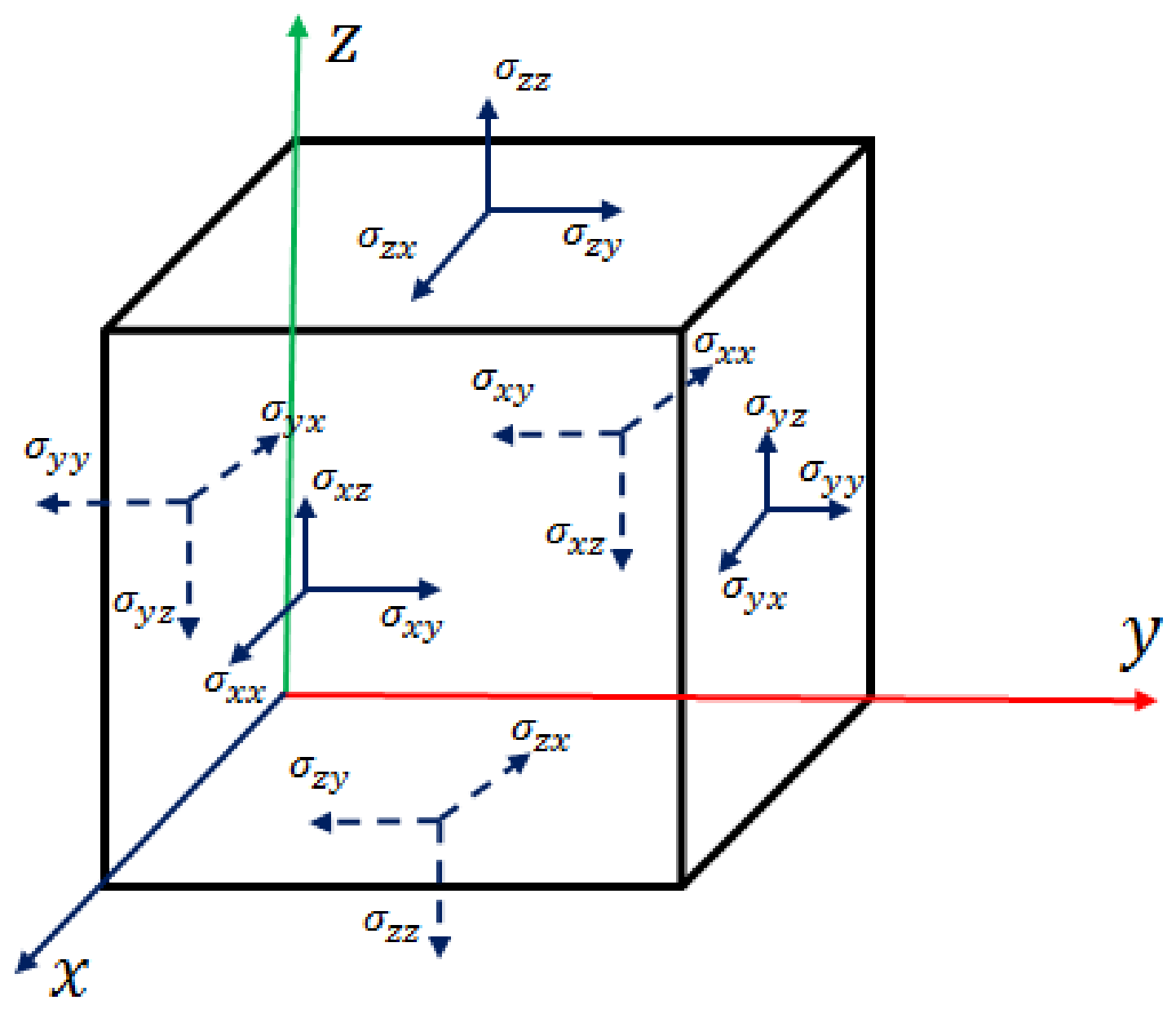

FIGURE 44: Six independent stress components at a point in a solid viewed on the surfaces of an infinitely small cubic block.

constants. The relationship between these three constants is

$$
G=\frac{E}{2(1+v)}
$$

That is to say, for any isotropic material, given any two of the three constants, the other one can be calculated using the above equation. 


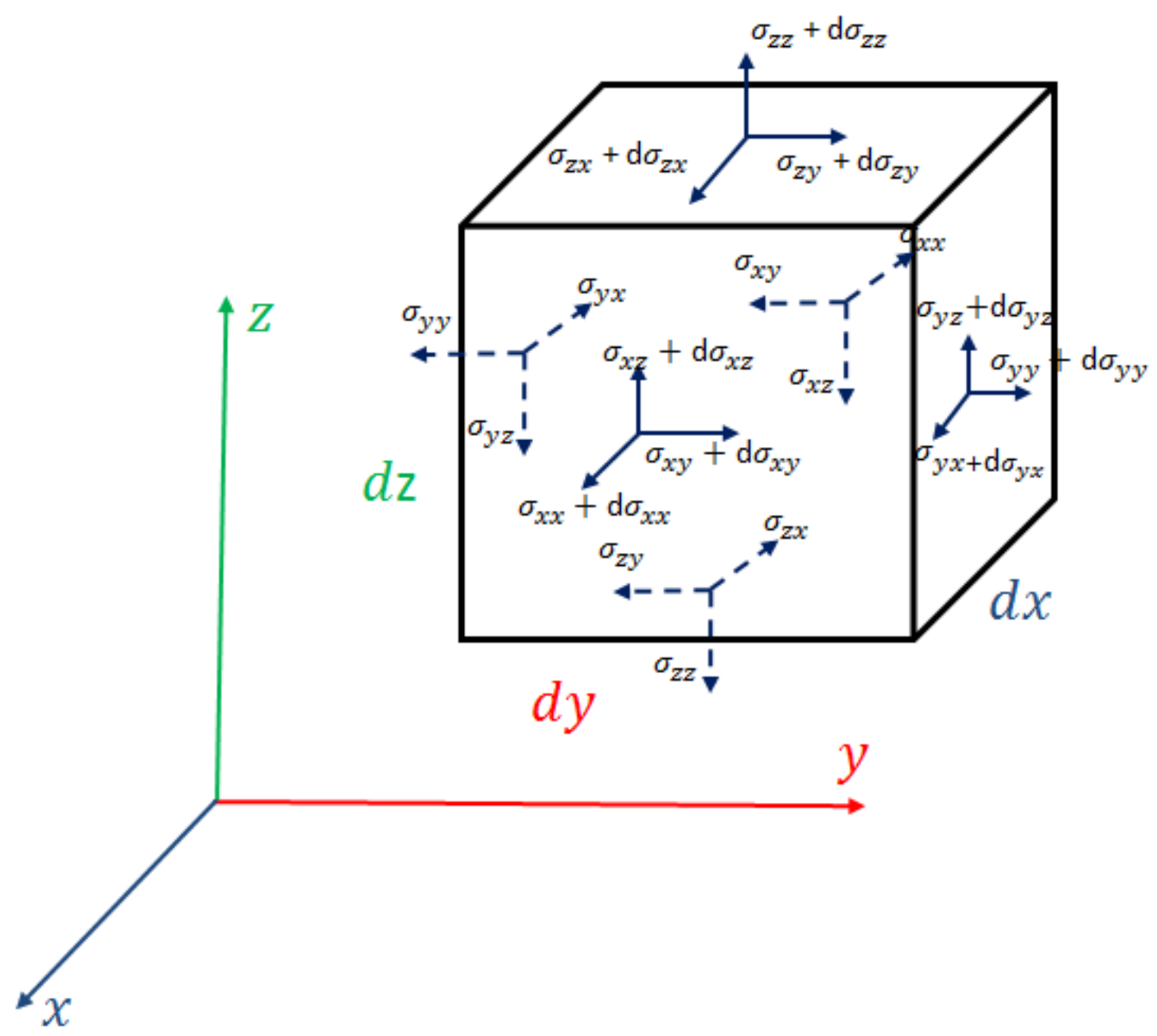

FIGURE 45: Stresses on an infinitely small block. Equilibrium equations are derived based on this state of stresses. 
3. Dynamic Equilibrium Equation

The formulation of the dynamic equilibrium equations depend on assuming an infinitely small block of solid (see Figure 45). In equilibrium case, The forces should be applied on all directions. Since this is a general, dynamic system, the inertial forces of the block is considered. The equilibrium of forces in the $x$ direction gives as follows:

$$
\begin{aligned}
\left(\sigma_{x x}+d \sigma_{x x}\right) d y d z-\sigma_{x x} d y d z+\left(\sigma_{y x}\right. & \left.+d \sigma_{y x}\right) d x d z-\sigma_{y x} d x d z \\
& +\left(\sigma_{z x}+d \sigma_{z x}\right) d x d y-\sigma_{z x} d x d y+f_{x}=\rho \ddot{u} d x d y d z
\end{aligned}
$$

where, $\rho \ddot{u} d x d y d z$ is the inertial force term, $f_{x}$ is the external body force applied at the centre of the small block and $d \sigma_{x x}, d \sigma_{y x}$ and $d \sigma_{z x}$ defined as follow:

$$
d \sigma_{x x}=\frac{\partial \sigma_{x x}}{\partial x} d x, \quad d \sigma_{y x}=\frac{\partial \sigma_{y x}}{\partial y} d y, \quad d \sigma_{z x}=\frac{\partial \sigma_{z x}}{\partial z} d z
$$

Hence, Equation 69 becomes one of the equilibrium equations, written as:

$$
\frac{\partial \sigma_{x x}}{\partial x}+\frac{\partial \sigma_{y x}}{\partial y}+\frac{\partial \sigma_{z x}}{\partial z}+f_{x}=\rho \ddot{u}
$$

Similarly, the equilibrium of forces in the $y$ and $z$ directions results as:

$$
\begin{aligned}
& \frac{\partial \sigma_{x y}}{\partial x}+\frac{\partial \sigma_{y y}}{\partial y}+\frac{\partial \sigma_{z y}}{\partial z}+f_{y}=\rho \ddot{v} \\
& \frac{\partial \sigma_{x z}}{\partial x}+\frac{\partial \sigma_{y z}}{\partial y}+\frac{\partial \sigma_{z z}}{\partial z}+f_{z}=\rho \ddot{w}
\end{aligned}
$$


From Equations 71 to 73 , the equilibrium equations can be written in matrix form as:

$$
\mathbf{L}^{T} \sigma+\mathbf{f}_{b}=\rho \ddot{\mathbf{U}}
$$

where $\mathbf{f}_{b}$ is the vector of external body forces in the $x, y$ and $z$ directions:

$$
\mathbf{f}_{b}=\left\{\begin{array}{l}
f_{x} \\
f_{y} \\
f_{z}
\end{array}\right\}
$$

\section{Boundary Conditions}

There are two types of boundary conditions: 1) displacement (essential). 2) force (natural) boundary conditions. The displacement boundary condition can be simply written as:

$$
u=\bar{u} \text { and/or } \quad v=\bar{v} \text { and } / \text { or } \quad w=\bar{w}
$$

on displacement boundaries. For most of the actual simulations, the displacement is used to describe the support or constraints on the solid models, and hence the prescribed displacement values are often zero. In such cases, the boundary condition is characterize as a homogenous boundary condition. Otherwise, they are inhomogeneous boundary conditions.

The force boundary condition is written as: 


$$
\mathbf{n} \sigma=\bar{t}
$$

on force boundaries, where $\mathbf{n}$ is given by

$$
\mathbf{n}=\left[\begin{array}{cccccc}
n_{x} & 0 & 0 & 0 & n_{z} & n_{y} \\
0 & n_{y} & 0 & n_{z} & 0 & n_{x} \\
0 & 0 & n_{z} & n_{y} & n_{x} & 0
\end{array}\right]
$$

in which $n_{i}(i=x, y, z)$ are cosines of the outwards normal on the boundary. A force boundary condition can also be both homogenous and inhomogeneous. If the condition is homogeneous, it implies that the boundary is a free surface.

\section{E. Materials and Methods}

1. Mesh generation(Pre-processing)

To perform FEA of human tooth, PDL, and bone deformation, preprocessing techniques are needed: image segmentation, mesh generation, and measurement of the mechanical properties. In this section, each one of these preprocessing techniques performed in this Chapter is explained in detail.

1) Image Segmentation: The triangular meshes of the training ensemble are obtained from a high resolution computer tomography scan of human invitro teeth. A Conebeam CT (KODAK 9000 3D Extraoral) scanner at a resolution of $0.2 \times 0.2 \times 0.2 \mathrm{~mm}$ is then used to models, where the Expectation-Maximization (EM) algorithm is used for segmentation [139]. 


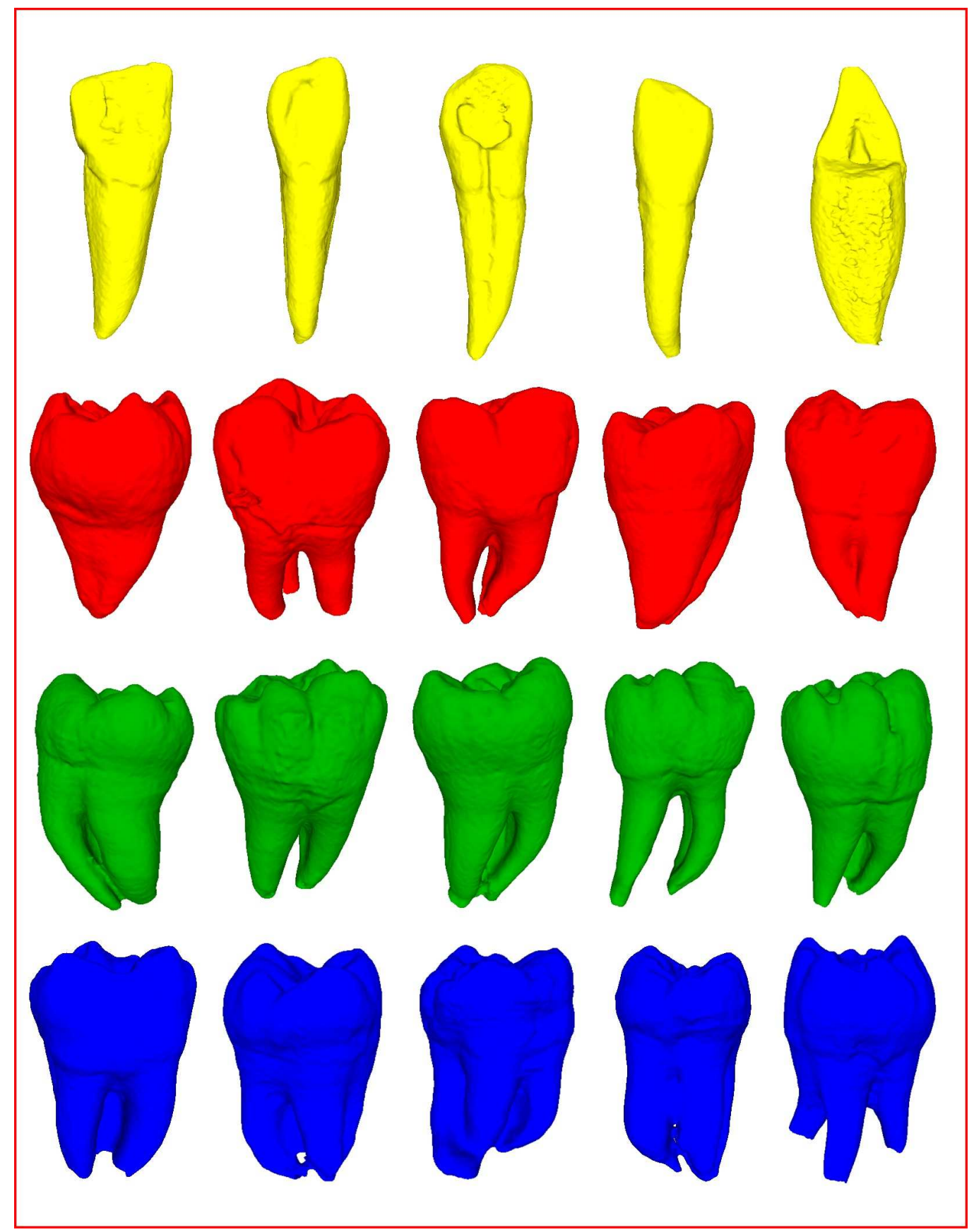

FIGURE 46: Ensemble of 20 models generated using the proposed method illustrated with different colors. First row: solid volumes generated from the CT scan of the anterior teeth. Second row: solid volumes generated from the CT scan of the mandibular third molars. Third row: solid volumes generated from the CT scan of the mandibular molars. Last row: solid volumes generated from the CT scan of the maxillary third molars 
2) Mesh Generation: Volumetric mesh generation depends on decomposing the object volume into a finite union of geometrically simple and bounded elements. In the context of the medical imaging, some mesh generators were created for image registration $[140,141]$. In this chapter, the interest is generating high quality tetrahedral meshes that conform to the input surface meshes. To carry out this aim, the images are firstly segmented using EM segmentation algorithm and then convert the segmented images into a stereolithographic file(STL) using VTK [142]. Then, the STLs are used to generate 3D solid meshes of the tooth (see Figure 46), PDL and cortical bone .

\section{FEA using FEBio}

1) Materials Properties: Equations are developed for each element in the FEM mesh and assembled together into a set of global equations that model the properties of the entire system. Table 9 [1-4] summarizes the mechanical properties of the enemal, dentin, PDL and cortical bone.

2) Loads and Boundary Conditions (LBC's): In this step, loads and boundary conditions defined on discreized models of different teeth in PreView ${ }^{13}$ [130].

3) Processing: In this step, FEA solver FEBio ${ }^{14}$ [130] is used to calculate displacement and stress due to loading conditions.

The 3D form of Hooke's law can be written as [143]:

\footnotetext{
${ }^{13}$ PreView is a finite element preprocessing software package. Its primary function is to set up the boundary conditions and material properties for finite element analysis with the software FEBio.

${ }^{14} \mathrm{FEBio}$ is a nonlinear finite element solver that is specifically designed for biomechanical applications.
} 


$$
\left(\begin{array}{c}
\sigma_{x} \\
\sigma_{y} \\
\sigma_{z} \\
\tau_{x y} \\
\tau_{y z} \\
\tau_{z x}
\end{array}\right)=\mathbf{D}\left(\begin{array}{c}
\varepsilon_{x} \\
\varepsilon_{y} \\
\varepsilon_{x} \\
\gamma_{x y} \\
\gamma_{y z} \\
\gamma_{z x}
\end{array}\right)
$$

where $\sigma$ and $\tau$ are the stress and shear stress respectively and $\varepsilon$ and $\gamma$ are the strain and shear strain respectively. $\mathbf{D}$ is called the stress/strain or constitutive matrix and is defined by:

$$
\mathbf{D}=\frac{E}{(1+v)(1-2 v)}
$$

$$
\times\left(\begin{array}{cccccc}
1-v & v & v & 0 & 0 & 0 \\
0 & 1-v & v & 0 & 0 & 0 \\
0 & 0 & 1-v & 0 & 0 & 0 \\
0 & 0 & 0 & \frac{1-2 v}{2} & 0 & 0 \\
0 & 0 & 0 & 0 & \frac{1-2 v}{2} & 0 \\
0 & 0 & 0 & 0 & 0 & \frac{1-2 v}{2}
\end{array}\right)
$$

where $E$ is defined as the modulus of elasticity (Young's modulus) and $v$ is Poisson's ratio. 
TABLE 9: Mechanical properties of human teeth (Youngs modulus and poissions ratio of the tooth) [1-4].

\begin{tabular}{|c|c|c|}
\hline Material property & Young's modulus(GPa) & Poisson's ratio \\
\hline \hline Enamel & 80 & 0.33 \\
Dentine & 18.6 & 0.31 \\
PDL & 0.0689 & 0.45 \\
Cortical bone & 1.37 & 0.30 \\
\hline
\end{tabular}

3. Visualization

Finally, the output of the FEBio is used as the input to PostView ${ }^{15}$ [130] to show the stress and displacement on the tooth.

Figure 40 summarizes the overall procedure used in the chapter to determine the stress and displacement in different teeth.

\section{F. Experimental Results and Discussions}

In order to evaluate the performance of the proposed approach, several experiments are carried out on real human teeth, other than the ones used for constructing the 3D models.

\section{Horizontal Forces}

Differences were determined for the displacement and stress distribution a five models (Anterior tooth, mandibular third molar, mandibular molar, maxillary third molar and two touched teeth) under different loading conditions.

\footnotetext{
${ }^{15} \mathrm{PostView}$ is a finite element (FE) post-processing application that is designed to view FEBio output files.
} 
In this study, simple horizontal orally directed forces, strengths $0.5 \mathrm{~N}$ to $3 \mathrm{~N}$ were applied on five different models. This force is simple tipping which was applied on the tooth crown, perpendicular to its longitudinal axis.

Figure 47 shows the stress and displacement distribution in four different models. Figure 47(a) illustrates the 3D solid mesh that we get it from the STL surface mesh and loaded with tipping force near the crown. Stress increases from crown to root as shown in Figure 47(b). Maximum stress is near the root and the maximum value of displacement near to the crown (see Figure 47(c)).

Figure 48 simulates the invivo force on the human teeth and how the movement of the tooth effects the neighboring teeth. Figure 48(a) shows the solid mesh for two neighborhood teeth. Figure 48(b) shows the effective stress due to the force $(3 N)$ on the left tooth in the opposite y-direction and the pressure of the right tooth on the left tooth.

\section{Vertical Forces}

In order to evaluate the performance of the proposed approach, several experiments are done on 250 real human teeth (40 anterior models, 125 mandibular third molars models, 40 mandibular molars models and 45 maxillary molars models). Differences were determined for the stress, displacement and pressure distribution between models (mandibular third molar, mandibular molar and maxillary) under different loading conditions(see Figure 49 to Figure 53).

In this study, simple vertical orally directed forces, strength overall $200 \mathrm{~N}$ was divided into three vectors (cusps)in some cases and four vectors on the others. This force was applied on the tooth crown, perpendicular to its occlusal surface. The $200 \mathrm{~N}$ load used in this chapter was selected, as average chewing force, which is supposed to be one third of the maximum biting force [144]. Under the compressive load, the axial test simulations 


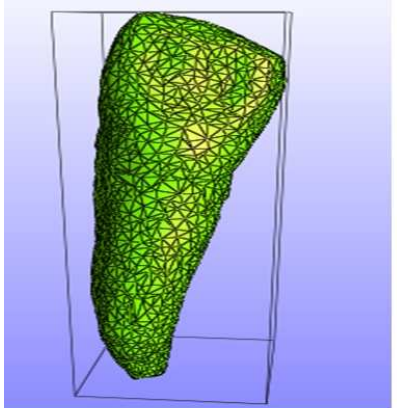

Anterior Tooth

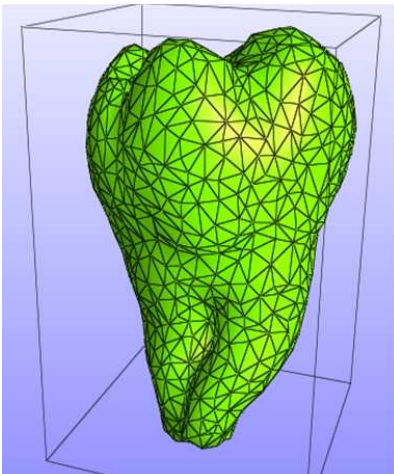

Mandibular Third Molar

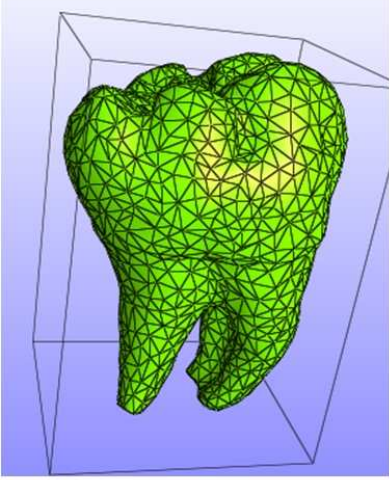

Mandibular Molar

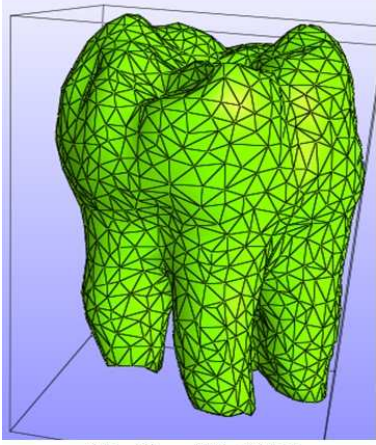

Maxillary Third Molar

(a)
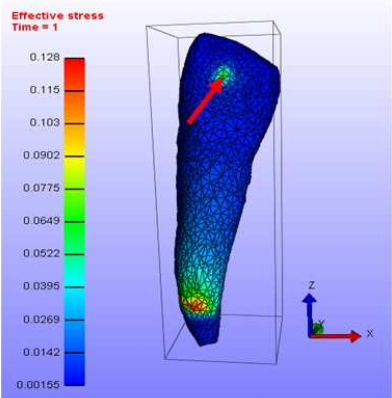

Force $(0.5 \mathrm{~N})$ in Y-Direction

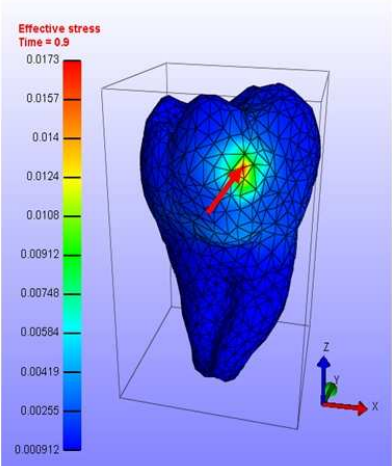

Force $(1 \mathrm{~N})$ in Y-Direction

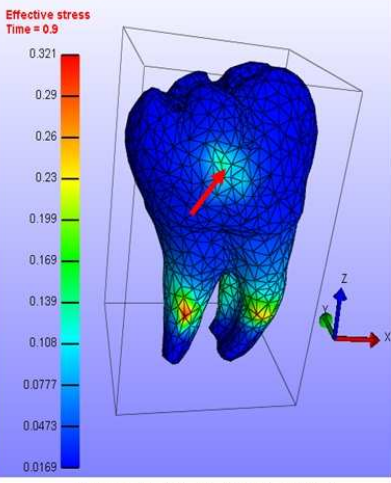

Force $(2 \mathrm{~N})$ in Y-Direction

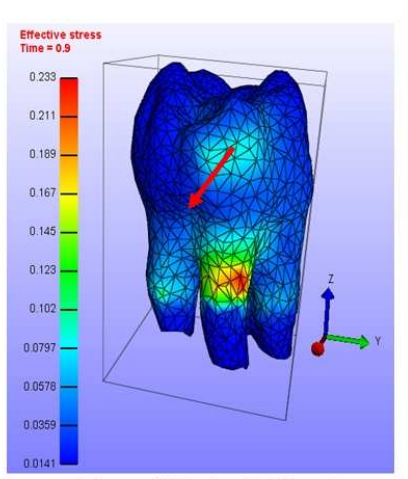

Force $(3 \mathrm{~N})$ in -X-Direction

(b)
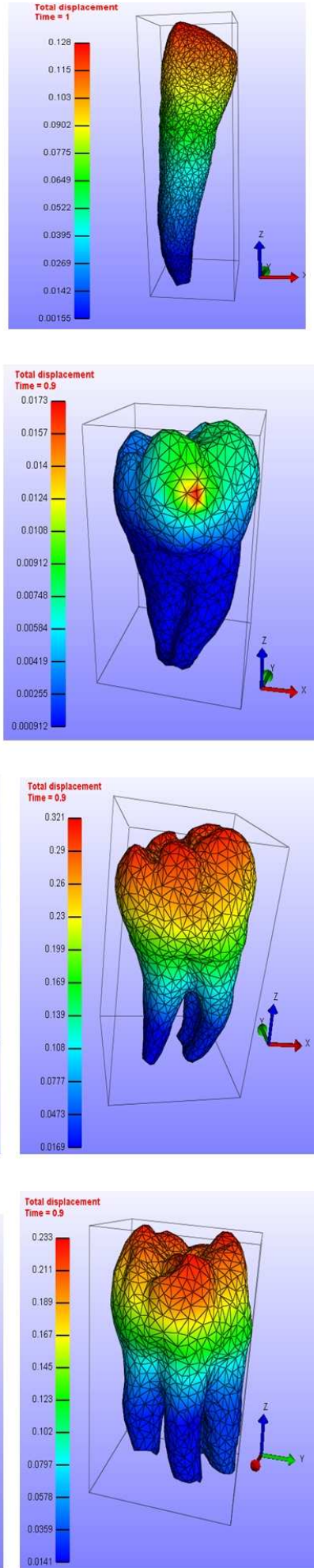

(c)

FIGURE 47: (a) Finite element mesh (solid mesh) with the material property and initial conditions. (b) Effective stress for the tooth due to orthodontic forces were depicted as concentrated force. (c) The correspondence total displacement for the anterior tooth 


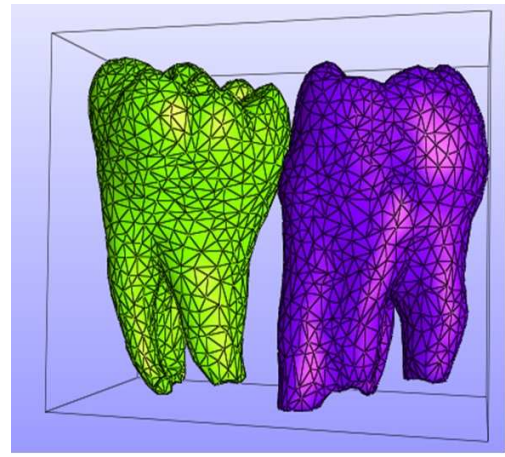

(a)

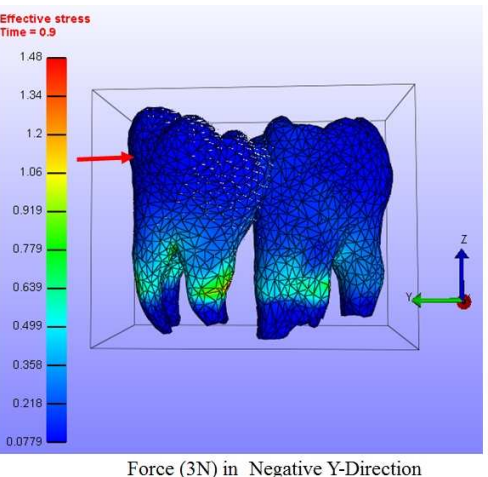

(b)

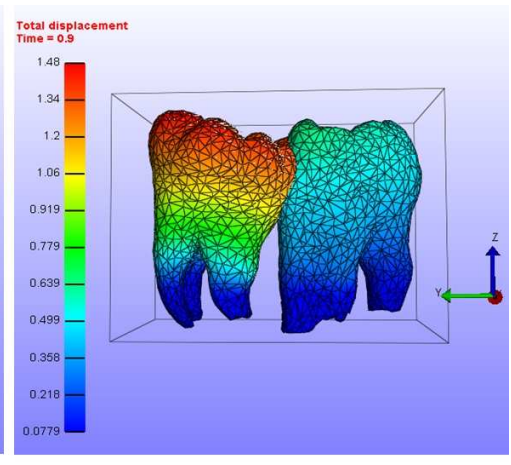

(c)

FIGURE 48: The mechanism of transferring the load from one tooth to its neighborhood: (a) Finite element meshes with the material property and initial conditions. (b) The effective stress (expressed in MPa) due to the force $(3 \mathrm{~N})$ on the left tooth in the opposite y-direction and the pressure of the right tooth on the left tooth. (c) The correspondence total displacement.

predicted that tooth fracture might occur between $700 \mathrm{~N}$ and $800 \mathrm{~N}$ [144]. In addition, the boundary conditions of each model simulated the contact with neighbouring teeth. In the first model (mandibular third molar with three roots), the stress values ranged from 0.408 to $4.08 \mathrm{KPa}$, the displacement values ranged from $0.165 \times 10^{-8}$ to $1.65 \times 10^{-8}$ and compression values range from $-1.65 \times 10^{3}$ to $0.0599 \times 10^{3}$ as shown in Figure 49 . In the second model (mandibular molar)(see Figure 50 and Figure 51), the stress values ranged from 0.222 to 4.43 $\mathrm{KPa}$, the displacement values ranged from $0.102 \times 10^{-8}$ to $1.48 \times 10^{-8}$ and compression values range from -11 to $1.35 \times 10^{3}$. In the third model (Maxillary third molar with two roots)(see Figure 52 and Figure 53), the stress values ranged from 0.325 to $3.61 \mathrm{KPa}$, the displacement values ranged from $0.187 \times 10^{-8}$ to $4.45 \times 10^{-8}$ and compression values range from -1.96 to $0.127 \times 10^{3}$.

\section{G. Summary}




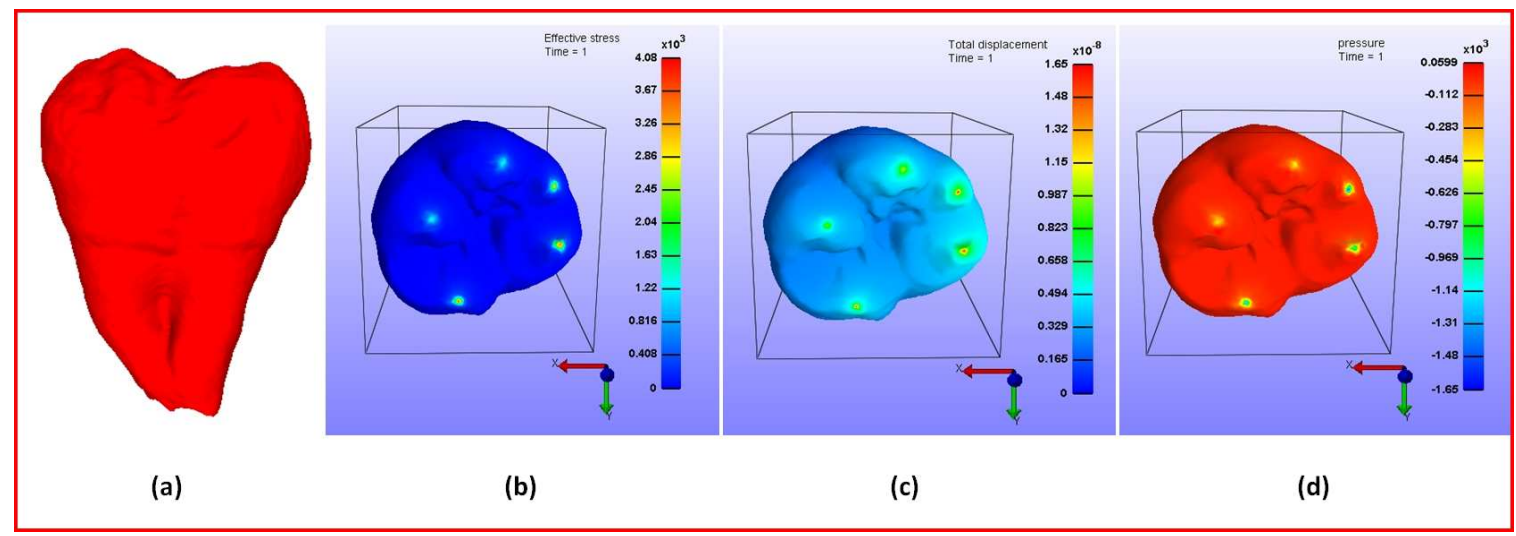

FIGURE 49: 3D model and stress, displacement and compression distributions in the mandibular third molar with cusp 4 points loading. (a) Finite element mesh (solid mesh) with the material property and initial conditions. (b) Effective stress for the tooth due to orthodontic $200 \mathrm{~N}$ tensile forces were depicted as four concentrated force in $-z$-direction (as a tensile force). (c) The correspondence total displacement. (d) The correspondence compression.

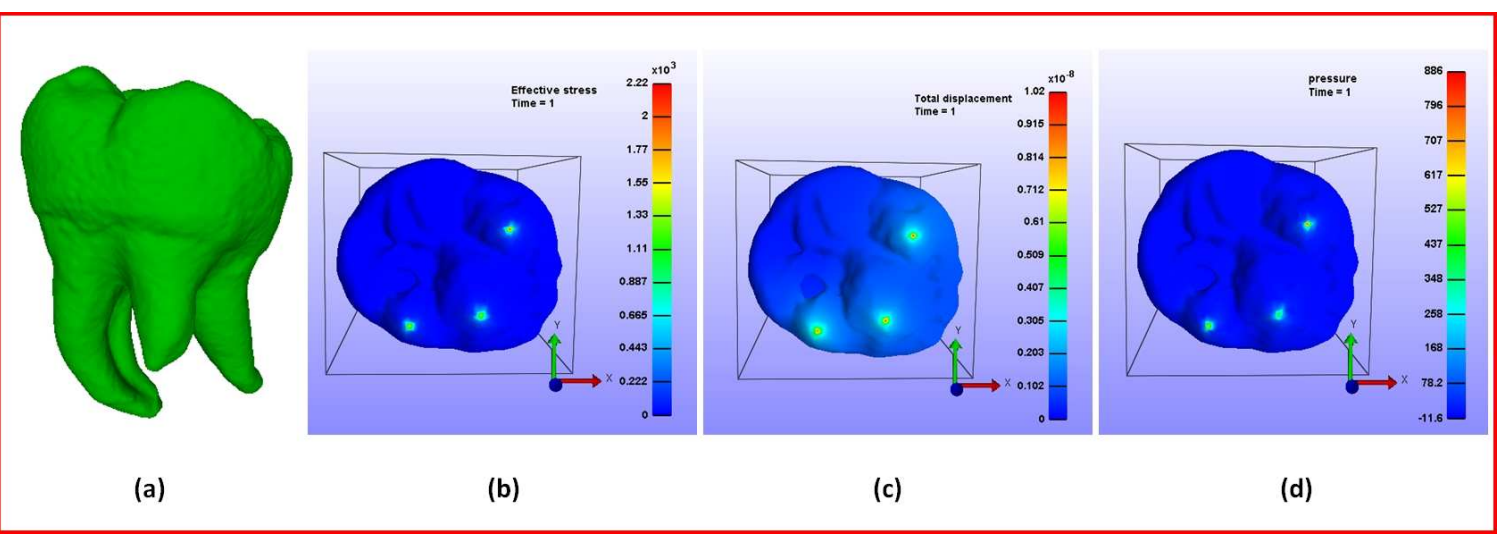

FIGURE 50: 3D model and stress, displacement and compression distributions in the mandibular molar (three roots) with cusp 3 points loading. (a) Finite element mesh (solid mesh) with the material property and initial conditions. (b) Effective stress for the tooth due to orthodontic $200 \mathrm{~N}$ tensile forces were depicted as four concentrated force (in $z$-direction (as a compression force). (c) The correspondence total displacement. (d) The correspondence compression. 


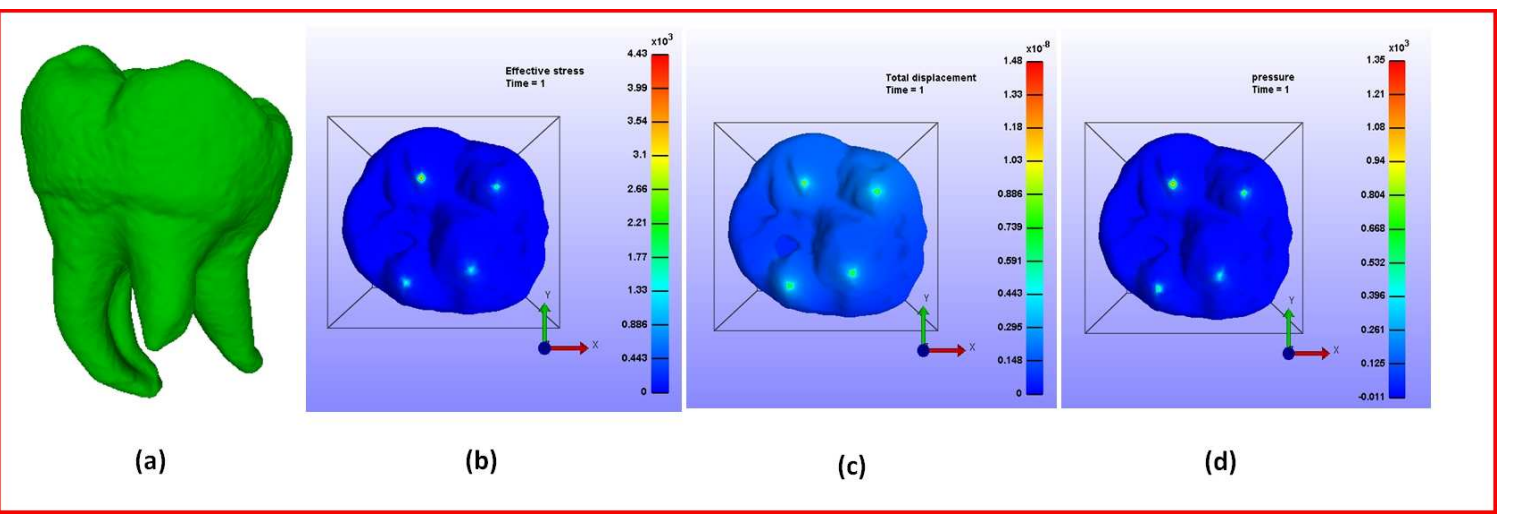

FIGURE 51: 3D model and stress, displacement and compression distributions in the mandibular molar (three roots) with cusp 4 points loading. (a) Finite element mesh (solid mesh) with the material property and initial conditions. (b) Effective stress for the tooth due to orthodontic $200 \mathrm{~N}$ tensile forces were depicted as four concentrated force in $z$-direction (as a compression force). (c) The correspondence total displacement. (d) The correspondence compression.

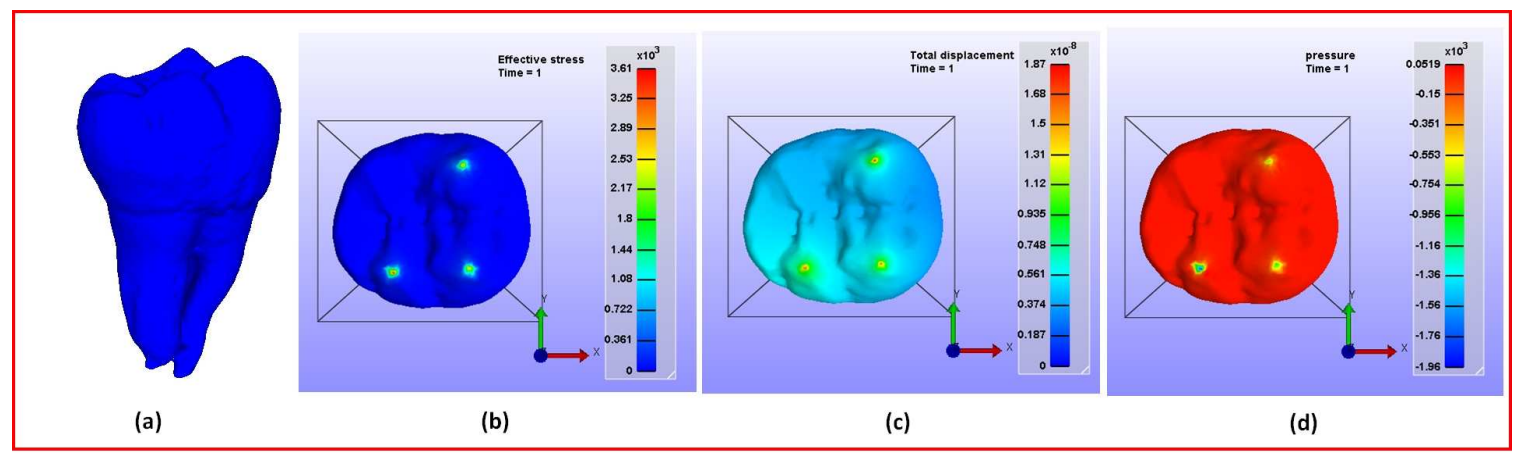

FIGURE 52: 3D model and stress, displacement and compression distributions in the maxillary 3rd molar (two roots) with cusp 3 points loading. (a) Finite element mesh (solid mesh) with the material property and initial conditions. (b) Effective stress for the tooth due to orthodontic $200 \mathrm{~N}$ tensile forces were depicted as four concentrated force in $-z$-direction (tensile force). (c) The correspondence total displacement. (d) The correspondence compression. 


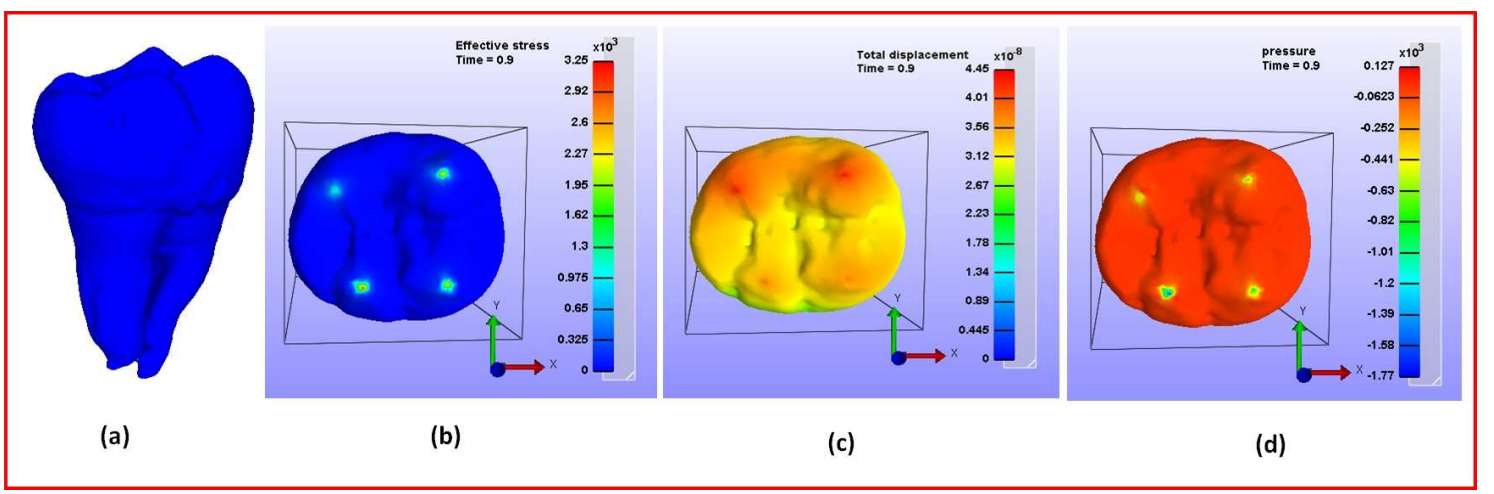

FIGURE 53: 3D model and stress, displacement and compression distributions in the maxillary 3rd molar (two roots) with cusp 4 points loading. (a) Finite element mesh (solid mesh) with the material property and initial conditions. (b) Effective stress for the tooth due to orthodontic $200 \mathrm{~N}$ tensile forces were depicted as four concentrated force in $-z$-direction. (c) The correspondence total displacement. (d) The correspondence compression.

In this chapter, an accurate three-dimensional CAD model is proposed from DICOM images and converted to STL. Then the STL file converted to volume (solid mesh) to use it further in FEA. 3D stress and displacements of different real teeth type are successfully carried out on Anterior tooth, mandibular third molar, mandibular molar and maxillary third molar models using open-source finite element solver, FEBio. The limitations of the experimental and analytical approaches used for stress and displacement analysis are overcome by using FEA tool benefits such as dealing with complex geometry and complex loading conditions. The effect of applying a force system to the two teeth is addressed where the movement of one tooth will exert force on neighboring teeth. Qualitative and quantitative analysis based on FEBio's progressive visual color scale, ranging from dark blue to red are illustrated. 


\section{CHAPTER IX}

\section{CONCLUSIONS AND FUTURE WORK}

This dissertation has presented 3D reconstruction using new SFS approaches and shape modeling with applications to dentistry. Moreover, it studied the orthodontic tooth movement using analytical and FEA methods.

\section{A. Summary of Contributions}

The findings from this dissertation make several contributions to the current literature which can be summarized as follows:

- Chapter III, a 3D library of teeth/jaw is built and is made it available for other's research.

- Chapter IV focused on the 3D surface reconstruction aspect for human teeth based on a single image. A more realistic formulation of the SFS problem is introduced by considering the image formation components: the camera, the light source, and the surface reflectance. We propose a non-Lambertian SFS algorithm under perspective projection which benefits from camera calibration parameters. The attenuation of illumination due to near-field imaging is taken into account.

- Chapter V proposed a novel approach for 3D surface reconstruction of the human jaw [20]. Due to the difficulties of setting up a data acquisition system inside the mouth, an intraoral camera is used to capture a sequence of calibrated images. These 
images are registered together to build a panoramic view of the jaw.

- Chapter VI, an approach for realistic 3D reconstruction of the human teeth is developed using shape from shading with statistical shape priors. The work has addressed several challenges including near illumination and camera perspective projection, while taking into account the deviation from the simplifying Lambertian assumption. The Oren-Nayar reflectance model is used for diffuse rough surfaces with the roughness parameter being physically measured by an optical surface profiler.

- Chapter VII enhanced the current algorithms presented in the previous chapter (VI). The approach developed in this chapter reconstructs the teeth from single image shading with 2D-PCA shape priors that have a more sophisticated reflectance model. The Oren-Nayar-Wolff model was used for modeling the surface reflectance.

- In Chapter VIII, an accurate three-dimensional CAD model is proposed from DICOM images and converted to STL. Then the STL file converted to volume (solid mesh) to use it further in FEA. 3D stress and displacements of different real teeth type are successfully carried out on Anterior tooth, mandibular third molar, mandibular molar and maxillary third molar models using open-source finite element solver, FEBio.

\section{B. Limitations and Suggested Future Directions}

The analysis also lead to a number of interesting observations, some of which may be considered as lines of future research.

- The next step is to investigate the fusion of SFS and SSFS where SFS provides the object-specific constructions while SSFS is perform shape recovery based on partial information. 
- Designing and developing algorithms to construct a complete 3D model of the human jaw that includes crows, roots, and gum information by fusing the $3 \mathrm{D}$ surface and teeth databases as well as X-ray images.

- Evaluation/validation of the reconstruction approach with respect to traditional methods for specific dental practices. 


\section{REFERENCES}

[1] RA Moga and CG Chiorean. Strain analysis of a human tooth with support tissues resorption. In Proceedings of the World Congress on Engineering, volume 2, 2013.

[2] Piotr Kowalczyk. Influence of the shape of the layers in photo-cured dental restorations on the shrinkage stress peaksfem study. Dental Materials, 25(12):e83-e91, 2009.

[3] U Andreaus, M Colloca, and Daniela Iacoviello. Coupling image processing and stress analysis for damage identification in a human premolar tooth. Computer methods and programs in biomedicine, 103(2):61-73, 2011.

[4] Beata Dejak, Andrzej Młotkowski, and Cezary Langot. Three-dimensional finite element analysis of molars with thin-walled prosthetic crowns made of various materials. Dental Materials, 28(4):433-441, 2012.

[5] Aly S Abdelrahim, Aly A Farag, Shireen Y Elhabian, and Moumen T El-Melegy. Shape-from-shading using sensor and physical object characteristics applied to human teeth surface reconstruction. IET Computer Vision, 8(1):1-15, 2014.

[6] C.N. Carter, R.J. Pusateri, Dongqing Chen, A.H. Ahmed, and A.A. Farag. Shape from shading for hybrid surfaces as applied to tooth reconstruction. In Image Processing (ICIP), 2010 17th IEEE International Conference on, pages 4049-4052, 2010 .

[7] B. Horn. Robot Vision. McGraw-Hill, 1986.

[8] R. Zhang, P. Tsai, J. E. Cryer, and M. Shah. Shape from shading: A survey. IEEE Transactions on Pattern Analysis and Machine Intelligence, 21(1):690-706, 1999.

[9] E. Prados, F. Camilli, and O. Faugeras. A unifying and rigorous shape from shading method adapted to realistic data and applications. Journal of Mathematical Imaging and Vision, 25:307-328, 2006.

[10] A. Tankus, N. Sochen, and Y. Yeshurun. Shape-from-shading under perspective projection. In International Journal of Computer Vision, 2005.

[11] S. Y. Yuen, Y. Y. Tsui, and C. K. Chow. A fast marching formulation of perspective shape from shading under frontal illumination. Pattern Recognit. Lett., 28:806-824, 2007.

[12] A. Ahmed and A. Farag. Shape from shading under various imaging conditions. In Proc. of IEEE Conference on Computer Vision and Pattern Recognition (CVPR'07), Minneapolis, MN, pages X1-X8, June 18-23, 2007. 
[13] Oliver Vogel, Michael Breuß, and Joachim Weickert. Perspective shape from shading with non-lambertian reflectance. In Proceedings of the 30th DAGM symposium on Pattern Recognition, pages 517-526, Berlin, Heidelberg, 2008. Springer-Verlag.

[14] Oliver Vogel, Levi Valgaerts, Michael Breu, and Joachim Weickert. Making shape from shading work for real-world images. In Joachim Denzler, Gunther Notni, and Herbert Se, editors, Pattern Recognition, volume 5748 of Lecture Notes in Computer Science, pages 191-200. Springer Berlin Heidelberg, 2009.

[15] M. Oren and S. Nayar. Generalization of the lambertian model and implications for machine vision. International Journal of Computer Vision, 14(3):227 - 251, 1995.

[16] H. Ragheb and E. Hancock. Surface normals and height from non-lambertian image data. In Second International Symposium on 3D Data Processing, Visualization and Transmission (3DPVT'04), Greece, 2004.

[17] J. D. Durou, M. Falcone, and M. Sagona. Numerical methods for shape-fromshading: A new survey with benchmarks. Computer Vision and Image Understanding, 109(1):22 - 43, 2008.

[18] S. M. Yamany, A. A. Farag, D. Tasman, and A. G. Farman. A 3-d reconstruction system for the human jaw using a sequence of optical images. IEEE Transactions on Medical Imaging, 19:538-547, May 2000.

[19] M. T. Ahmed, A. Eid, and A. A. Farag. Human jaw reconstruction: New approach and improvements. In International Conference on Medical Image Computing and Computer-Assisted Intervention (MICCAI'01), The Netherlands, Oct. 2001.

[20] A. S. Abdelrahim, M. A. Abdelrahman, H. Abdelmunim, A. Farag, and M. Miller. Novel image-based $3 \mathrm{~d}$ reconstruction of the human jaw using shape from shading and feature descriptors. In Proceedings of the British Machine Vision Conference, pages 41.1-41.11. BMVA Press, 2011.

[21] A. Abdelrahim, M. El-Melegy, and A. Farag. Realistic 3d reconstruction of the human teeth using shape from shading with shape priors. In IEEE Computer Vision and Pattern Recognition Workshops (CVPRW), pages 64 -69, june 2012.

[22] E. Prados and O. Faugeras. Shape from shading: a well-posed problem? In International Conference on Computer Vision and Pattern Recognition CVPR05, San Diego, CA, USA, June 2005.

[23] P. R. Garant. a theory of fibroblastic maintenance of the periodontal ligament. 47(7):380-390.

[24] M. N. Ahmed, S. M. Yamany, E. E. Hemayed, and A. A. Farag. 3d reconstruction of the human jaw from a sequence of images. In IEEE International Conference on Computer Vision and Pattern Recognition (CVPR97), Puerto Rico, pages 646-653, June, 1997. 
[25] S. M. Yamany, A. A. Farag, A. Farman, and D. Tasman. Robust 3-d reconstruction of the human jaw from video images. In International Conference on Medical Image Computing and Computer-Assisted Intervention (MICCAI99), pages 778-787, Cambridge, England, September 1999.

[26] A. H. Abdelrehim and A. A. Farag. A new formulation for shape from shading for non-lambertian surfaces. In International Conference on Computer Vision and Pattern Recognition CVPR06, pages 1817-1824, NY, USA, 2006.

[27] D. Scharstein and R. Szelisk. A taxonomy and evaluation of dense two-frame stereo correspondence algorithms. International Journal for Computer Visionl, 47(1):7-42, May 2002.

[28] S. Roy and I. J. Cox. A maximum-flow formulation of the n-camera stereo correspondence problem. In Proceedings of International Conference on Computer Vision (ICCV98), pages 492-499, Jan 1998.

[29] M. Okutomi and T. Kanade. A multiple baseline stereo. IEEE Transactions on Pattern Analysis and Machine Intelligencel, 15:353-453, April 1993.

[30] H. Baker and T. Binford. Depth from edge and intensity based stereo. In Proceedings of Seventh International Joint Conference on Artificial Intelligence, Vancouver, pages 631-636, 1981.

[31] D. Marr and T. Poggio. Cooperative computation of stereo disparity. Science, 194:283-287, October 1976.

[32] J. Garding. Direct estimation of shape from texturen. IEEE Transaction on Pattern Analysis and Machine Intelligence (PAMI), 15(11):1202-1207, 1993.

[33] D. Blostien and N. Ahuja. Shape from texture: integrating texture-element extraction and surface estimation. IEEE Transaction on Pattern Analysis and Machine Intelligence (PAMI), 11(12):1233-1251, 1989.

[34] J. Oliensis. Exact two-image structure from motion. PAMI, 24:1618-1633, 2002.

[35] C. Tomasi and T. Canade. Shape and motion from image streams under orthography: a factorization method. IJCV, 9:137-154, 1992.

[36] B.K.P. Horn and E. J. Weldon. Direct methods for recovering motion. IJCV, 2(1):51$76,1988$.

[37] B. K. P. Horn and B. G. Schunck. Determining optical flow. AI, 17:185-203, 1981.

[38] Berthold K. P. Horn. Height and gradient from shading. Int. J. Comput. Vision, 5(1):37-75, 1990.

[39] R. Kimmel and A. M. Bruckstein. Tracking level sets by level sets: a method for solving the shape from shading problem. Computer Vision and Image Understanding, 62(2):47-58, 1995.

[40] G. Q. W. and G. Hirzinger. Learning shape from shading by a multiplayer network. In IEEE Transactions Neural Networks, pages 985-995, July 1996. 
[41] Alex P. Pentland. Extract shape from shading. In MIT Media Lab, 2nd ed., 1988.

[42] P. S. Tsai and M. Shah. A fast linear shape from shading. In Proc. IEEE Computer Vision and Pattern Recog- nition Conf. (CVPR), Urbana, Illinois, pages 734-736, june 1992.

[43] R. Zhang, P. Tsai, J. Cryer, and M. Shah. Analysis of shape from shading techniques. In Proc. CVPR, Seattle, pages 377-384, June 1994.

[44] K. M. Lee and J. Kuo. Shape from shading with perspective projection. Computer Vision, Graphics, and Image Processing: Image Understanding, 59:202-212, 1994.

[45] J. K. Hasegawa and C. L. Tozzi. Shape from shading with perspective projection and camera calibration. Computer and Graphics., 20(3):351-364, 1996.

[46] K. M. Lee and J. Kuo. Shape from shading with generalized reflectance map model. Computer Vision and Image Understanding, 67:143-160, August 1997.

[47] S.M. Seitz and C.R. Dyer. Photorealistic scene reconstruction by voxel coloring. In Computer Vision and Pattern Recognition, Proceedings., IEEE Computer Society Conference on, pages 1067-1073, 1997.

[48] K.N. Kutulakos and S.M. Seitz. A theory of shape by space carving. In Computer Vision, 1999. The Proceedings of the Seventh IEEE International Conference on, volume 1, pages 307-314 vol.1, 1999.

[49] W. Culbertson, T. Malzbender, and G. Slabaugh. Generalized voxel coloring. In International Workshop on Vision Algorithms, Corfu, Greece, pages 100-115, 1999.

[50] E. Grimson. From images to surfaces: A computational study of the human early vision system. MIT Press, Cambridge, MA, 1981.

[51] O. Faugeras. Three-Dimensional Computer Vision (Artificial Intelligence). MIT Press, Cambridge, MA, 1993.

[52] R. Hartley and A. Zisserman. Multiple View Geometry in computer vision. Cambridge University Press., 2000.

[53] F. Tong and B. V. Funt. Removing specularity from color images for shape from shading. Science, 14:275-290, 1989.

[54] B. Prescott and G. McLean. line-based correction of radial lens distortion. Graph. Models and Image Process, 59(1), Jan. 1997.

[55] M. T. Ahmed and A. A. Farag. Differential methods for non-metric calibration of camera lens distortion. In IEEE International Conference on Computer Vision and Pattern Recognition (CVPR2001), Hawaii, pages 477-482, December 2001.

[56] M. T. El-Melegy and Aly A. Farag. Nonmetric lens distortion calibration: Closedform solutions, robust estimation and model selection. In in Proc IEEE International Conference of Computer Vision (ICCV), 2003. 
[57] G. Xu and Z. Zhang. Epipolar geometry in stereo. In Motion and Object Recognition, Kluwer Academic, The Netherlands., 1996.

[58] M. Pollefeys, R. Koch, and L. Van Gool. Self-calibration and metric reconstruction in spite of varying and unknown internal camera parameters. In Computer Vision, 1998. Sixth International Conference on, pages 90-95, 1998.

[59] J. Oliensis. Fast and accurate self-calibration. In Computer Vision, 1999. The Proceedings of the Seventh IEEE International Conference on, volume 2, pages 745752 vol.2, 1999.

[60] B.K.P. Horn. Obtaining shape from shading information. In P. H. Winston, editor, The Psychology of Computer Vision, chapter 4, pages 115-155. McGraw-Hill, New York, 1975.

[61] B. K. P. Horn. Image intensity understanding. Artificial Intelligence ., 8(2):201-231, 1977.

[62] B. Horn. Robot Vision. MIT Press, 1986.

[63] B. Horn. Shape from Shading. MIT Press, 1989.

[64] Q. Zheng and R. Chellappa. Estimation of illuminant direction, albedo, and shape from shading. IEEE Transactions on Pattern Analysis and Machine Intelligence ., 13(7):680-702, July 1991.

[65] K. M. Lee and C.-C. J. Kuo. Shape from shading with a linear triangular element surface model. IEEE Transactions on Pattern Analysis and Machine Intelligence ., 15(8):815-822, Aug. 1993.

[66] M. Bichsel and A. P. Pentland. A simple algorithm for shape from shading. In In Computer Vision and Pattern Recognition., pages 459-465, 1992.

[67] Chia-Hoang Lee and Azriel Rosenfeld. Improved methods of estimating shape from shading using the light source coordinate system. Artificial Intelligence, 26(2):125$143,1985$.

[68] A. P. Pentland. Local shading analysis. IEEE Transactions on Pattern Analysis and Machine Intelligence (PAMI), PAMI-6(2):170-187, 1984.

[69] P.S. Tsai and M. Shah. Shape from shading using linear approximation. Image and Vision Computing J., 12(8):487-498, 1994.

[70] Ron Kimmel and Alfred M. Bruckstein. Global shape from shading. Comput. Vis. Image Underst., 62(3):360-369, Nov. 1995.

[71] W. Zhao and R. Chellappa. Face recognition using symmetric shape from shading. In Proceedings of the IEEE Computer Society Conference on Computer Vision and Pattern Recognition ., 4:286-293, Hilton Head, SC, June 1993.

[72] R. Kimmel and J. A. Sethian. Optimal algorithm for shape from shading and path planning. Journal of Mathematical Imaging and Vision., 14(3):237-244, 2001. 
[73] J. A. Sethian. Level Set Methods and Fast Marching Methods. Evolving Interfaces in Computational Geometry, Fluid Mechanics, Computer Vision, and Materials Science. Cambridge Monograph on Applied and Computational Mathematics. Cambridge University Press, 2 edition., 1999.

[74] O. Faugeras E. Prados and E. Rouy. Shape from shading and viscosity solutions. In A. Heyden, G. Sparr, M. Nielsen, and P. Johansen, editors, 7th European Conference on Computer Vision, 2:790- 804, May.

[75] J-D. Durou, M. Falcone, and M. Sagona. A survey of numerical methods for shape from shading. 2004-2-r, Institut de Recherche en Informatique de Toulouse (IRIT), 2004.

[76] K. Ikeuchi and B.K.P. Horn. Numerical shape from shading and occluding boundaries. Artificial Intelligence, 17(1-3):141-184, 1981.

[77] M.J. Brooks and B.K.P. Horn. Shape and source from shading. In Proceedings of the International Joint conference on Artificial Intelligence, pages 932-936, 1985.

[78] R.T. Frankot and R. Chellappa. A method for enforcing integrability in shape from shading algorithms. IEEE Trans. on PAMI, 10(4):439-451, 1988.

[79] C. Loop and Zhengyou Zhang. Computing rectifying homographies for stereo vision. In Computer Vision and Pattern Recognition, 1999. IEEE Computer Society Conference on., volume 1, pages -131 Vol. 1, 1999.

[80] Y. Boykov, O. Veksler, and R. Zabih. Fast approximate energy minimization via graph cuts. In Computer Vision, 1999. The Proceedings of the Seventh IEEE International Conference on, volume 1, pages 377-384 vol.1, 1999.

[81] C.K. Liang, Y.C. Shih, and H. H. Chen. Light field analysis for modeling image formation. IEEE Transactions on Image Processing, 20(2):446 - 460, Feb. 2011.

[82] A. Abdelrahim, A. Shalaby, S. Elhabian, J. Graham, and A. Farag. A 3d reconstruction of the human jaw from a single image. In IEEE International Conference on Image Processing (ICIP), pages 3622-3626, 2013.

[83] S. Elhabian, A. Abdelrahim, A. Farag, D. Tasman, W. Aboelmaaty, and A. Farman. Clinical crowns shape reconstruction - an image-based approach. In Biomedical Imaging (ISBI), 2013 IEEE 10th International Symposium on, pages 93-96, 2013.

[84] H. Hassan, A. El-Baz, A. A. Farag, A. Farman, D. Tazman, and M. Miller. A complete volumetric 3d model of the human jaw. In Proc. of Computer Assisted Radiology and Surgery (CARS), Berlin, Germany, pages 1244-1249, June 2005.

[85] H. Chui and A. Rangarajan. A new point matching algorithm for non-rigid registration. Comput. Vis. Image Underst., 89:114-141, February 2003.

[86] Paul J Besl and Neil D McKay. Method for registration of 3-d shapes. In RoboticsDL tentative, pages 586-606. International Society for Optics and Photonics, 1992. 
[87] Yang Chen and Gérard Medioni. Object modelling by registration of multiple range images. Image and vision computing, 10(3):145-155, 1992.

[88] Zhengyou Zhang. Iterative point matching for registration of free-form curves and surfaces. International journal of computer vision, 13(2):119-152, 1994.

[89] H. L. Mitchell and R. G. Chadwick. Challenges of photogrammetric intra-oral tooth measurement. In The International Archives of the Photogrammetry, Remote Sensing and Spatial Information Sciences, Kyoto, Japan, pages 779-782, 2008.

[90] B.K.P. Horn. Shape from Shading: A Method for Obtaining the Shape of a Smooth Opaque Object from One View. $\mathrm{PhD}$ thesis, Massachusetts Inst. of Technology, Cambridge, Massachusetts, 1970.

[91] E. Prados, F. Camilli, and O. Faugeras. A unifying and rigorous shape from shading method adapted to realistic data and applications. J. Math. Imaging Vis., 25:307328, 2006.

[92] S. K. Nayar L. B.Wolff and M. Oren. Improved diffuse reflection models for computer vision. IJCV, 30(1):55-71, 1998.

[93] M. Oren and S. Nayar. Generalization of lambert's reflectance model. In Computer Graphics, 28(Annual Conference Series), pages 239-246, 1994.

[94] Hossein Ragheb and Edwin R. Hancock. Surface radiance correction for shape from shading. Pattern Recognition, 38:1574-1595, 2005.

[95] B. K. P. Horn and M. J. Brooks. The variational approach to shape from shading. Comput. Vision Graph. Image Process., 33:174-208, February 1986.

[96] M. Elad, A. Tal, and S. Ar. Content based retrieval of vrml objects: an iterative and interactive approach. In Proceedings of the sixth Eurographics workshop on Multimedia 2001, pages 107-118, New York, NY, USA, 2002. Springer-Verlag New York, Inc.

[97] R. Tyrrell Rockafellar and R. J-B Wets. Variational Analysis. Springer-Verlag, 2005. pg.117.

[98] D. Laurendeau, L. Guimond, and D. Poussart. A computer-vision technique for the acquisition and processing of 3-d profiles of dental imprints: an application in orthodontics. Medical Imaging, IEEE Transactions on, 10(3):453-461, 1991.

[99] C. Bernard, A. Fournier, J. M. Brodeur, H. Naccache, and R. Guay. Computerized diagnosis in orthodontics. In Proc. 66th Gen. Session Int. Assoc. Dental Res. Montreal, 1988.

[100] F. P. van der Linden, H. Boersma, T. Zelders, K. A. Peters, and J. H. Raben. Threedimensional analysis of dental casts by means of optocom. In J. Dent. Res., volume 51, pages 1100-1972, 1972. 
[101] S. N. Bhatia and V. E. Harrison. Operational performance of the traveling microscope in the measurement of dental casts. In Br. J. Orthod., volume 14, pages 147$153,1987$.

[102] K. Tanaka, A. A. Lowe, and R. DeCou. Operational performance of the reflex monograph and its applicability to the three-dimensional analysis of dental casts. In Amer. J. Orthod., volume 83, pages 304-305, 1983.

[103] A. A. Goshtasby, S. Nambala, W. G. deRijk, and S. D. Campbell. A system for digital reconstruction of gypsum dental casts,. In IEEE Transactions on Medical Imaging, volume 16, pages 664-674, Oct 1997.

[104] J. M. Morel and G. Yu. Asift: A new framework for fully affine invariant image comparison. SIAM Journal on Imaging Sciences, 2(2):438-469, 2009.

[105] M. A. Fischler and R. C. Bolles. Random sample consensus, a paradigm for model fitting with applications to image analysis and automated cartography. In International Journal of Computer Vision, 24:381-395, 1981.

[106] Charles Fox. An introduction to the calculus of variations. Courier Dover Publications, 1987.

[107] George B Arfken, Hans J Weber, and Donald Spector. Mathematical methods for physicists. American Journal of Physics, 67:165-169, 1999.

[108] K. M. Lee and J. Kuo. Shape from shading with perspective projection. Computer Vision, Graphics, and Image Processing: Image Understanding ., 59:202-212, 1994.

[109] M. J. Grenness, J. E. Osborn, and M. J. Tyas. Mapping tooth surface loss with a fixed-base stereo-camera,. In Photogrammetric Record, volume 23, pages 194-207, 2008 .

[110] J. Atick, P. Griffin, and A. Redlich. Statistical approach to shape from shading: Reconstruction of 3D face surfaces from single 2D images. Neural Computation, 8:1321-1340, 1997.

[111] W. Smith and E.R. Hancock. Recovering facial shape using a statistical model of surface normal direction. IEEE Trans. on PAMI, 28(12):1914 -1930, Dec. 2006.

[112] I. Kemelmacher-Shlizerman and R. Basri. 3D face reconstruction from a single image using a single reference face shape. IEEE Trans. on PAMI, 33(2):394 -405, Feb. 2011.

[113] Xuesong Lu, Su Zhang, He Su, and Yazhu Chen. Mutual information-based multimodal image registration using a novel joint histogram estimation. Computerized Medical Imaging and Graphics, 32(3):202-209, 2008.

[114] Dimitris Samaras and Dimitris Metaxas. Incorporating illumination constraints in deformable models for shape from shading and light direction estimation. IEEE Trans. Pattern Anal. Mach. Intell., 25(2):247-264, 2003. 
[115] A. Shalaby, M. Aslan, H. Abdelmunim, and A. A. Farag. 2d pca-based shape prior for level sets segmentation framework of the vertebral body. In Biomedical Engineering Conference (CIBEC), pages 134-137, 2012.

[116] A. Shalaby, A. Mahmoud, E. Mostafa, A. Abdoulmalek, and A. A. Farag. Segmentation framework of vertebral body using 2d-pca. In Proc. of 15th Saudi Technical Exchange Meeting, pages 81-85, 2012.

[117] J. Yang, D. Zhang, A. Frangi, and Jing yu Yang. Two-dimensional pca: a new approach to appearance-based face representation and recognition. Pattern Analysis and Machine Intelligence, IEEE Transactions on, 26(1):131 -137, jan. 2004.

[118] A. Farag, S. Elhabian, A. Abdelrahim, W. Aboelmaaty, A. Farman, and D. Tasman. Model-based human teeth shape recovery from a single image with unkwon illumination. In MICCAI Medical Computer Vision workshop(MVC), 2012.

[119] P.M. Cattaneo, M. Dalstra, and B. Melsen. The finite element method: a tool to study orthodontic tooth movement. 84:428-433, May 2005.

[120] A. Van Schepdael, J. Vander Sloten, and L. Geris. A mechanobiological model of orthodontic tooth movement. In Biomechanics and Modeling in Mechanobiology,, November 2012.

[121] C. Provatidis. An analytical model for stress analysis of a tooth in translation. 39:1361-1381, 2001.

[122] An Van Schepdael. Biomechanical and mechanobiological modelling of orthodontic tooth movement (biomechanische en mechanobiologische modellering van orthodontische tandbewegingen). status: published, 2011.

[123] Johnny G Cailleteau, Monty R Rieger, and J Akin. A comparison of intracanal stresses in a post-restored tooth utilizing the finite element method. Journal of endodontics, 18(11):540-544, 1992.

[124] HJA Meijer, FJM Starmans, F Bosman, and WHA Steen. A comparison of three finite element models of an edentulous mandible provided with implants. Journal of oral rehabilitation, 20(2):147-157, 1993.

[125] Photini Kamposiora, George Papavasilious, Stephen C Bayne, and David A Felton. Finite element analysis estimates of cement microfracture under complete veneer crowns. The Journal of prosthetic dentistry, 71(5):435-441, 1994.

[126] JS Rees and PH Jacobsen. Modelling the effects of enamel anisotrophy with the finite element method. Journal of oral rehabilitation, 22(6):451-454, 1995.

[127] David C Holmes, Ana M Diaz-Arnold, and James M Leary. Influence of post dimension on stress distribution in dentin. The Journal of prosthetic dentistry, 75(2):140147, 1996.

[128] JS Rees. An investigation into the importance of the periodontal ligament and alveolar bone as supporting structures in finite element studies. Journal of Oral Rehabilitation, 28(5):425-432, 2001. 
[129] Major M Ash. Occlusion: reflections on science and clinical reality. The Journal of prosthetic dentistry, 90(4):373-384, 2003.

[130] Sa Maas, JB Ellis, GA Ateshian, and JA Weiss. Febio: Finite elements for biomechanics. Journal of Biomechanical Engineering, 134(1):011005, 2012.

[131] A. V. Schepdael. Biomechanical and mechanobiological modelling of orthodontic tooth movement. In PhD thesis, KU Leuven, November 2011.

[132] M. Gei, F. Genna, and D. Bigoni. An interface model for the periodontal ligament. 124(5):538-546, 2002.

[133] A. I. Zhurov, G. Limbert, D. P. Aeschlimann, and J. Middleton. A constitutive model for the periodontal ligament as a compressible transversely isotropic viscohyperelastic tissue. 10(3):223-235, 2007.

[134] Y. Kojima and H. Fukui. Numerical simulation of canine retraction by sliding mechanics. 127:542-551, 2005.

[135] Y. Kojima, T. Mizuno, and H. Fukui. A numerical simulation of tooth movement produced by molar uprighting spring. 132:630-638, 2007.

[136] Y. Kojima, T. Mizuno, S. Umemura, and H. Fukui. A numerical simulation of orthodontic tooth movement produced by a canine retraction spring. 26:561-567, 2007.

[137] A. Van Schepdael, J. Vander Sloten, and L. Geris. A visco-elastic model for the prediction of orthodontic tooth movement. July 2012.

[138] SS Quek and GR Liu. Finite Element Method: A Practical Course: A Practical Course. Butterworth-Heinemann, 2003.

[139] C. Carson, S. Belongie, H. Greenspan, and J. Malik. Blobworld: image segmentation using expectation-maximization and its application to image querying. Pattern Analysis and Machine Intelligence, IEEE Transactions on, 24(8):1026-1038, 2002.

[140] J. M. Sullivan, G. Charron Jr., and K. D. Paulsen. A three-dimensional mesh generator for arbitrary multiple material domains. Finite Elements Anal. Design, 25:219241, 1997.

[141] H. Hartmann and F. Kruggel. A fast algorithm for generating 3d finite element meshes from magnetic resonance tomograms. in Proc. IEEE Workshop on Biomedical Image Analysis, pages 184-192, 1998.

[142] http://www.vtk.org.

[143] Daray L. Logan. Afirst course in the Finite Element Method, third edition. Wadsworth Group, Pacific Grove,CA,USA, 2002.

[144] Josipa Borčić, Robert Antonić, Miranda Muhvić Urek, Nikola Petričević, Petra Nola-Fuchs, Amir Čatić, and Ivica Smojver. 3-d stress analysis in first maxillary premolar. Collegium antropologicum, 31(4):1025-1029, 2007. 


\section{Appendix A}

\section{A. Derivatives of the 3D point vector}

From Eq.(25)

$$
\begin{gathered}
\mathbf{M}=\mathbf{B}^{-1}(s \mathbf{m}-\mathbf{b}) \\
\mathbf{M}_{x}=\mathbf{B}^{-1}\left(s_{x} \mathbf{m}+s\left[\begin{array}{l}
1 \\
0 \\
0
\end{array}\right]\right), \quad \mathbf{M}_{y}=\mathbf{B}^{-1}\left(s_{y} \mathbf{m}+s\left[\begin{array}{l}
0 \\
1 \\
0
\end{array}\right]\right)
\end{gathered}
$$

where $\mathbf{M}_{x}$ is the first derivative of the $3 \mathrm{D}$ point $\mathbf{M}$ with respect to $x$, and $\mathbf{M}_{y}$ is the first derivative of the 3D point $\mathbf{M}$ with respect to $y$.

$$
\begin{gathered}
\mathbf{M}_{x y}=\mathbf{B}^{-1}\left(s_{x y} \mathbf{m}+s_{x}\left[\begin{array}{l}
0 \\
1 \\
0
\end{array}\right]+s_{y}\left[\begin{array}{l}
1 \\
0 \\
0
\end{array}\right]\right), \\
\mathbf{M}_{y x}=\mathbf{B}^{-1}\left(s_{y x} \mathbf{m}+s_{x}\left[\begin{array}{l}
0 \\
1 \\
0
\end{array}\right]+s_{y}\left[\begin{array}{l}
0 \\
1 \\
0
\end{array}\right]\right)
\end{gathered}
$$

where $\mathbf{M}_{x y}$ is the first derivative of $\mathbf{M}_{x}$ with respect to $y$, and $\mathbf{M}_{y x}$ is the first derivative of $\mathbf{M}_{y}$ with respect to $x$. 


$$
\begin{gathered}
\mathbf{M}_{x x}=\mathbf{B}^{-1}\left(s_{x x} \mathbf{m}+2 s_{x}\left[\begin{array}{l}
1 \\
0 \\
0
\end{array}\right]\right), \\
\mathbf{M}_{y y}=\mathbf{B}^{-1}\left(s_{y y} \mathbf{m}+2 s_{y}\left[\begin{array}{l}
0 \\
1 \\
0
\end{array}\right]\right)
\end{gathered}
$$

where $\mathbf{M}_{x x}$ is the second derivative of the $3 \mathrm{D}$ point $\mathbf{M}$ with respect to $x$, and $\mathbf{M}_{y y}$ is the second derivative of the $3 \mathrm{D}$ point $\mathbf{M}$ with respect to $y$.

$$
\begin{gathered}
\mathbf{M}_{x x x}=\mathbf{B}^{-1}\left(s_{x x x} \mathbf{m}+3 s_{x x}\left[\begin{array}{l}
1 \\
0 \\
0
\end{array}\right]\right), \\
\mathbf{M}_{y y y}=\mathbf{B}^{-1}\left(s_{y y y} \mathbf{m}+3 s_{y y}\left[\begin{array}{l}
0 \\
1 \\
0
\end{array}\right]\right)
\end{gathered}
$$

where $\mathbf{M}_{x x x}$ is the third derivative of the $3 \mathrm{D}$ point $\mathbf{M}$ with respect to $x$, and $\mathbf{M}_{y y y}$ is the third derivative of the 3D point $\mathbf{M}$ with respect to $y$. 


$$
\begin{gathered}
\mathbf{M}_{x x x x}=\mathbf{B}^{-1}\left(s_{x x x x} \mathbf{m}+4 s_{x x}\left[\begin{array}{l}
1 \\
0 \\
0
\end{array}\right]\right), \\
\mathbf{M}_{y y y y}=\mathbf{B}^{-1}\left(s_{y y y y} \mathbf{m}+4 s_{y y}\left[\begin{array}{l}
0 \\
1 \\
0
\end{array}\right]\right)
\end{gathered}
$$

where $\mathbf{M}_{x x x x}$ is the fourth derivative of the $3 \mathrm{D}$ point $\mathbf{M}$ with respect to $x$, and $\mathbf{M}_{y y y y}$ is the fourth derivative of the 3D point $\mathbf{M}$ with respect to $y$.

\section{B. Brightness Constraint Derivatives}

From Eq.(55) we assume:

$$
f_{1}=(I-R)^{2}
$$

and the surface reflectance :

$$
R=\frac{\mathbf{V} \cdot \mathbf{L}}{\sqrt{\mathbf{V}^{T} \mathbf{V}}}
$$

where $\mathbf{V}=\mathbf{M}_{x} \times \mathbf{M}_{y}$

$$
\frac{\partial \varepsilon_{1}}{\partial s}=\frac{\partial f_{1}}{\partial s}-\frac{\partial}{\partial x}\left[\frac{\partial f_{1}}{\partial s_{x}}\right]-\frac{\partial}{\partial y}\left[\frac{\partial f_{1}}{\partial s_{y}}\right]
$$

where $\frac{\partial \varepsilon_{1}}{\partial s}$ is the first derivative of $\partial \varepsilon_{1}$ with respect to $s$.

$$
\frac{\partial f_{1}}{\partial s}=-2(I-R)\left(\frac{\left(\mathbf{V}^{T} \mathbf{V}\right)\left(\frac{\partial \mathbf{V}}{\partial s}\right)-\mathbf{V}\left(\mathbf{V}^{T} \frac{\partial \mathbf{V}}{\partial s}\right)}{\left(\mathbf{V}^{T} \mathbf{V}\right)^{1.5}}\right) \cdot \mathbf{L}
$$




$$
\begin{aligned}
& \frac{\partial f_{1}}{\partial s_{x}}=-2(I-R)\left(\frac{\left(\mathbf{V}^{T} \mathbf{V}\right)\left(\frac{\partial \mathbf{V}}{\partial s_{x}}\right)-\mathbf{V}\left(\mathbf{V}^{T} \frac{\partial \mathbf{V}}{\partial s_{x}}\right)}{\left(\mathbf{V}^{T} \mathbf{V}\right)^{1.5}}\right) \cdot \mathbf{L} \\
& \frac{\partial f_{1}}{\partial s_{y}}=-2(I-R)\left(\frac{\left(\mathbf{V}^{T} \mathbf{V}\right)\left(\frac{\partial \mathbf{V}}{\partial s_{y}}\right)-\mathbf{V}\left(\mathbf{V}^{T} \frac{\partial \mathbf{V}}{\partial s_{y}}\right)}{\left(\mathbf{V}^{T} \mathbf{V}\right)^{1.5}}\right) \cdot \mathbf{L}
\end{aligned}
$$

$$
\begin{aligned}
\frac{\partial \mathbf{V}}{\partial s}=\frac{\partial \mathbf{M}_{x}}{\partial s} \times \mathbf{M}_{y}+\mathbf{M}_{x} \times \frac{\partial \mathbf{M}_{y}}{\partial s} \\
=\mathbf{B}^{-1}\left[\begin{array}{c}
1 \\
0 \\
0
\end{array}\right] \times \mathbf{M}_{y}+\mathbf{M}_{x} \times \mathbf{B}^{-1}\left[\begin{array}{l}
0 \\
1 \\
0
\end{array}\right] \\
\frac{\partial \mathbf{V}}{\partial s_{x}}=\frac{\partial \mathbf{M}_{x}}{\partial s_{x}} \times \mathbf{M}_{y}+\mathbf{M}_{x} \times \frac{\partial \mathbf{M}_{y}}{\partial s_{x}}
\end{aligned}
$$

$$
=\left(\mathbf{B}^{-1} \mathbf{m}\right) \times \mathbf{M}_{y}
$$

$$
\frac{\partial}{\partial x}\left(\frac{\partial \mathbf{V}}{\partial s_{x}}\right)=\left(\mathbf{B}^{-1}\left[\begin{array}{l}
1 \\
0 \\
0
\end{array}\right]\right) \times \mathbf{M}_{y}+\left(\mathbf{B}^{-1} \mathbf{m}\right) \times \mathbf{M}_{y x}
$$

$$
\begin{aligned}
\frac{\partial \mathbf{V}}{\partial s_{y}} & =\frac{\partial \mathbf{M}_{x}}{\partial s_{y}} \times \mathbf{M}_{y}+\mathbf{M}_{x} \times \frac{\partial \mathbf{M}_{y}}{\partial s_{y}} \\
& =\mathbf{M}_{x} \times\left(\mathbf{B}^{-1} \mathbf{m}\right)
\end{aligned}
$$

$$
\frac{\partial}{\partial y}\left(\frac{\partial \mathbf{V}}{\partial s_{y}}\right)=\mathbf{M}_{x y} \times\left(\mathbf{B}^{-1} \mathbf{m}\right)+\mathbf{M}_{x} \times\left(\mathbf{B}^{-1}\left[\begin{array}{l}
0 \\
1 \\
0
\end{array}\right]\right)
$$




$$
\begin{gathered}
\mathbf{V}_{x}=\mathbf{M}_{x x} \times \mathbf{M}_{y}+M_{x} \times \mathbf{M}_{y x} \\
\mathbf{V}_{y}=\mathbf{M}_{x y} \times \mathbf{M}_{y}+\mathbf{M}_{x} \times \mathbf{M}_{y y}
\end{gathered}
$$

1. Smoothness Constraint Derivatives

From Eq.(28) set:

$$
f_{2}=\mathbf{M}_{x x}^{T} \mathbf{M}_{x x}+\mathbf{M}_{y y}^{T} \mathbf{M}_{y y}
$$

Then,

$$
\begin{gathered}
\frac{\partial \varepsilon_{2}}{\partial s}=-\frac{\partial}{\partial x}\left[\frac{\partial f_{2}}{\partial s_{x}}\right]-\frac{\partial}{\partial y}\left[\frac{\partial f_{2}}{\partial s_{y}}\right] \\
+\frac{\partial^{2}}{\partial x^{2}}\left[\frac{\partial f_{2}}{\partial s_{x x}}\right]+\frac{\partial^{2}}{\partial y^{2}}\left[\frac{\partial f_{2}}{\partial s_{y y}}\right] \\
\frac{\partial \varepsilon_{2}}{\partial s}=-4 \mathbf{M}_{x x x}^{T} \mathbf{B}^{-1}\left[\begin{array}{c}
1 \\
0 \\
0
\end{array}\right]-4 \mathbf{M}_{y y y}^{T} \mathbf{B}^{-1}\left[\begin{array}{l}
0 \\
1 \\
0
\end{array}\right] \\
+2 \mathbf{M}_{x x x x}^{T} \mathbf{B}^{-1} \mathbf{m}+2 \mathbf{M}_{y y y y}^{T} \mathbf{B}^{-1}
\end{gathered}
$$

\section{Integrability Constraint Derivatives}


From Eq.(29) set:

$$
\begin{aligned}
f_{3} & =\left(\mathbf{M}_{x y}-\mathbf{M}_{y x}\right)^{T}\left(\mathbf{M}_{x y}-\mathbf{M}_{y x}\right) \\
& =\left(s_{x y}-s_{y x}\right)^{2}\left(\mathbf{B}^{-1} \mathbf{m}\right)^{T}\left(\mathbf{B}^{-1} \mathbf{m}\right)
\end{aligned}
$$

Then,

$$
\begin{array}{r}
\frac{\partial \varepsilon_{3}}{\partial s}=\frac{\partial^{2}}{\partial x \partial y}\left[\frac{\partial f_{3}}{\partial s_{x y}}\right]+\frac{\partial^{2}}{\partial y \partial x}\left[\frac{\partial f_{3}}{\partial s_{y x}}\right] \\
=2\left(\mathbf{B}^{-1} \mathbf{m}\right)^{T}\left(\mathbf{B}^{-1} \mathbf{m}\right)\left(s_{x y y x}-s_{y x y x}-s_{x y x y}+s_{y x x y}\right)
\end{array}
$$




\section{CURRICULUM VITAE}

Name: Aly Abdelrahim Resume

Contact Information

Department of Electrical and Computer Engineering

University of Louisville

Mobile: +1-502-876-2122

Computer Vision and Image Processing Laboratory

2211 South Brook

Lutz Hall, Room \#6, Louisville, KY 40292, USA

E-mail: aly.abdelrahim@louisville.edu

Objective Pursuit of advanced research and development in computer vision, image processing, and medical imaging as a postdoctoral fellow.

Research Interests My research/development interests lie in the fields of computer vision, image understanding and pattern recognition. My primary focus is 3D shape reconstruction, shape-from-X, statistical shape analysis, and medical imaging.

\section{Career Profile}

- Have the will and the desire to learn.

- Like to be responsible. 
- Capable to work for long continuous period under pressure, in time sensitive and fast paced environment.

- Performed successfully in a team setting as well as individually.

\section{Education}

University of Louisville,Louisville, Kentucky, USA

- Thesis Topic: Three-Dimensional Modeling of the Human Jaw/Teeth Using Optics and Statistics

- GPA : 3.8 out of 4

- Advisor: Professor Aly A. Farag

- Thesis Committee:

- Professor Aly A. Farag, ECE Department and Director of CVIP-Lab@UofL

- Professor James H. Graham, Chairman of ECE Department

- Professor Sahoo, Prasanna K., Professor in A\&S Mathematics, University of Louisville

- Associate Professor Tamer Inanc, ECE Department

- Asstistant Professor Amirali Zandinejad, Dental school, University of Louisville

- Area of Study: Computer Vision and Image Processing

\section{Assiut University,Assiut, Egypt}

M.Sc., Faculty of Engineering, January 2007

- Thesis Topic: Image Retrieval Based on Content 
- Advisors: Professor Yousef B. Mahdy, Professor Hesham Abdel akhfar

- Area of Study: Image Processing

B.Sc., Faculty of Engineering, May 2001

- GPA: Very Good With Honors

- Project Grade: Excellent

\author{
Academic Appointments Graduate Research Assistant \\ August 2009 to present \\ Department of Electrical and Computer Engineering, \\ University of Louisville
}

\title{
Research and Development:
}

- Human jaw surface reconstruction from optical imaging for orthodontic treatments

Graduate Teaching Assistant

June 2002 to July 2007

Faculty of Computers and Information,

Assiut University

\section{Activities:}

- Lecturing classes and labs.

- Helping students throughout their study (office hours) 
- Helping students with their thesis projects

- Grading students' assignments

- Grading midterm exams

- Participating in the faculty's projects such as e-learning projects

\section{Refereed Journal Publications}

- Aly Abdelrahim, Aly Farag, Shireen Elhabian, and Moumen El-Melegy. Shapefrom-Shading using Sensor and Physical Object Characteristics Applied to Human Teeth Surface Reconstruction. IET-Computer Vision 2013.

- Yousef B. Mahdy, Aly Abdelrahim, Image Retrieval Based on Content, ICGST International Journal on Graphics, Vision and Image Processing (GVIP), Volume 6, Issue 1, July 2006.

\section{Conference Publications}

- Moumen El-Melegy, Aly Abdelrahim, and Aly Farag. "Better Shading for Better Shape Recovery", Computer Vision and Pattern Recognition 2014, accepted to appear.

- Aly Abdelrahim and Aly Farag. "Three-Dimensional Modeling and Stress Analysis in Dental Biomechanics using Finite Element Analysis”, ICIP, 2014, under review.

- Eslam Mostafa, Aly Abdelrahim, Shireen Elhabian and Aly Farag. "STATISTICAL MORPHABLE MODEL FOR HUMAN TEETH RESTORATION", ICIP, 2014, under review. 
- Aly Abdelrahim, Ahmed Shalaby, Moumen El-Melegy and Aly Farag, 2D-PCA Shape Models: Application to 3D Reconstruction of the Human Teeth from a Single Image, MICCAI Workshop on Medical Computer Vision (MCV2013).

- Abdelrahim, Aly; Abdelmunim, Hossam; Graham, James; Farag, Aly, "Novel variational approach for the perspective shape from shading problem using calibrated images," Image Processing (ICIP), 2013 20th IEEE International Conference on , vol., no., pp.2562,2566, 15-18 Sept. 2013

- Abdelrahim, Aly; Shalaby, Ahmed; Elhabian, Shireen; Graham, James; Farag, Aly, "A 3D reconstruction of the human jaw from a single image," Image Processing (ICIP), 2013 20th IEEE International Conference on , vol., no., pp.3622,3626, 15-18 Sept. 2013

- Aly Abdelrehim, Mostafa Abderahman, Hossam Abdelmunim, Aly Farag, and Mike Miller. Novel Image-Based 3D Reconstruction of the Human Jaw using Shape from Shading and Feature Descriptors. The 22nd British Machine Vision Conference, (BMVC), vol. 41, pp. 1-11, Aug 2011.

- Aly Abdelrahim, Moumen El-Melegy, and Aly Farag, Realistic 3D reconstruction of the human teeth using shape from shading with shape priors, Computer Vision and Pattern Recognition Workshops (CVPRW), pp.64-69, June 2012.

- Aly Abdelrahim, Aly Farag, Shireen Elhabian, Eslam Mostafa, and Wael Aboelmaaty, Occlusal surface reconstruction of human teeth from a single image based on object and sensor physical characteristics, International Conference on Image Processing (ICIP), pp.1793-1796, Oct. 2012.

- Shireen Elhabian,Aly Abdelrahim, Aly Farag, David Tazman, Wael Aboelmaaty and Allan Farman. Clinical Crowns Shape Reconstruction - An Image-based Approach. International Symposium on Biomedical Imaging: From Nano to Macro (ISBI) 2013. 
- Aly Farag, Shireen Elhabian, Aly Abdelrahim, Wael Aboelmaaty, Allan Farman and David Tazman. Model-based Human Teeth Shape Recovery from a Single Optical Image With Unknown Illumination. MICCAI Medical Computer Vision Workshop (MCV) 2012.

- Aly Abdelrahim, Mostafa Abdelrahman, Ali Mahmoud, Aly Farag, "Image retrieval based on content and image compression," IEEE International Conference on Multimedia Technology (ICMT), pp. 6696 - 6703, July 2011.

- Ali Mahmoud, Aly Farag, Aly Abdelrahim, Said Elnoubi, "A channel estimation technique in OFDM systems using no pilots," IEEE International Conference on Multimedia Technology (ICMT), pp. 6680 - 6683, July 2011.

\section{Honors and Awards}

- Outstanding student award, graduate student 2014 .

- Graduate Dean's Citation award 2014.

- IEEE Signal Processing Society Travel Grants, ICIP 2013.

- IEEE Computer Vision and Pattern Recognition (CVPR) Doctoral Consortium grant, 2013.

- ECE Outstanding Graduate Student Award 2013, Department of Electrical and Computer Engineering, University of Louisville, Louisville, Kentucky, USA.

- ECE Theobald Scholarship Award during the 2013 Honors \& Awards, University of Louisville, Louisville, Kentucky, USA.

- 2 place, $5^{\text {th }}$ Annual Graduate Student Research Symposium 2013, University of Louisville, Louisville, Kentucky, USA.

- Graduate Student Scholarship, University of Louisville, Fall 2011-Fall 2012. 
- Top student in class throughout my undergraduate education in Egypt

Application Areas Image-based rendering, 3D shape recovery, and medical imaging

Skills Programming Languages and Toolkits

- $\mathrm{C}$ and $\mathrm{C}++$

- Matlab

- $\mathrm{C} \#$

- Java

- Visulization Toolkit (VTK)
Fair and In Progress

Highly Competent

Fair and In Progress

Basic

Fair and In Progress

\section{Software}

Microsoft Office

Latex

Microsoft Visual Studio

Mathematica
Highly Competent

Highly Competent

good

Basic

Areas of Research Focus Mathematics:

- Real and complex analysis, differential geometry, linear algebra, multilinear algebra, and spherical harmonics

\section{Signal Processing:}


- Signal and image processing, probability, random variables, stochastic processes, information theory, subspace learning, pattern recognition and machine learning

\section{Computer Vision:}

- Shape-from-shading, statistical shape-from-shading, stereo reconstruction and image formation

\section{Medical Imaging:}

- Statistical shape analysis, image segmentation and image processing

\section{Relevant Coursework:}

- Electronic circuits,

- Control System Principles,

- Autonomous Robots,

- Communications and modulation,

- Measurements,

- Digital Signal Processing,

- Computer Vision,

- Image Processing,

- Database,

- Data structure,

- Computer graphics, 
- Networks,

- Digital Design,

- Computer Architecture

- Microprocessor.

\section{Languages}

- Arabic

- English

- French
Mother Tongue

Very Good

Basic

Hobbies: sports, fishing, collecting stamps, electricity, mechanics and carpentry 